University of Tennessee Health Science Center

UTHSC Digital Commons

\title{
Cephalometric Evaluation of Mara Therapy in the Treatment of Class II Malocclusions
}

Tiffany Rachelle McClaran

University of Tennessee Health Science Center

Follow this and additional works at: https://dc.uthsc.edu/dissertations

Part of the Orthodontics and Orthodontology Commons

\section{Recommended Citation}

McClaran, Tiffany Rachelle , "Cephalometric Evaluation of Mara Therapy in the Treatment of Class II Malocclusions" (2010). Theses and Dissertations (ETD). Paper 174. http://dx.doi.org/10.21007/ etd.cghs.2010.0206.

This Thesis is brought to you for free and open access by the College of Graduate Health Sciences at UTHSC Digital Commons. It has been accepted for inclusion in Theses and Dissertations (ETD) by an authorized administrator of UTHSC Digital Commons. For more information, please contact jwelch30@uthsc.edu. 


\title{
Cephalometric Evaluation of Mara Therapy in the Treatment of Class II Malocclusions
}

\begin{abstract}
The effectiveness of functional appliances is a debatable topic in the orthodontic literature, primarily because the benefits have not been consistently reproduced among clinical studies. The present study was a retrospective cephalometric analysis of patients with Class II, division 1 malocclusions at the start of treatment. There were 51 children treated by a single clinician with a MARA in combination with Edgewise appliances. The comparison group consisted of subjects treated with fixed Edgewise appliances only from the University of Tennessee, Department of Orthodontics. Subjects in the two treatment groups were matched on a one-to-one basis for sex and for five cephalometric variables (SNA, SNB, ANB, NAP, FMA) to ensure comparability. The key question was whether the cephalometric results at the end of treatment were comparable between the two groups. The MARA subjects were treated on an average of 2.8 years compared to the Edgewise group with a treatment time of 2.5 years. The Edgewise group had a larger proportion of premolars extracted for treatment compared to the MARA subjects, $69 \%$ and $4 \%$, respectively. Cephalometrically, ANB was on average $1^{\circ}$ smaller in the MARA group. Overall, the lower incisors proclined in both treatment groups during treatment. Similar skeletodental endpoints were achieved in both groups regardless of treatment mechanics. We conclude that either treatment are viable treatment options in the treatment of Class II division 1 malocclusions.
\end{abstract}

\section{Document Type}

Thesis

\section{Degree Name}

Master of Dental Science (MDS)

\section{Program}

Orthodontics

\section{Research Advisor}

Edward F. Harris, Ph.D.

\section{Keywords}

Class II Malocclusion, Functional appliance, MARA

\section{Subject Categories}

Dentistry | Medicine and Health Sciences | Orthodontics and Orthodontology 


\title{
CEPHALOMETRIC EVALUATION OF MARA THERAPY IN THE
} TREATMENT OF CLASS II MALOCCLUSIONS

\author{
A Thesis \\ Presented for \\ The Graduate Studies Council \\ The University of Tennessee \\ Health Science Center
}

\author{
In Partial Fulfillment \\ Of the Requirements for the Degree \\ Master of Dental Science \\ From The University of Tennessee
}

By

Tiffany Rachelle McClaran, D.D.S.

May 2010 
Copyright $@ 2010$ by Tiffany Rachelle McClaran All rights reserved 


\section{ACKNOWLEDGEMENTS}

First and foremost I would like to thank my mother for taking such good care of me from heaven during all of my endeavors, without her strength and watchful eye I could not have done this. Secondly, I would like to thank my husband, Matt, for his never ending support throughout this thesis experience and graduate program. I would also like to thank Dr. Edward F. Harris for his expertise, continued guidance and leadership. Without the help of Dr. Harris, this project would not be possible. I would also like to thank Dr. Jere L. Yates and Dr. Joe L. Wasson for serving on my committee. Their direction and support were invaluable. 


\begin{abstract}
The effectiveness of functional appliances is a debatable topic in the orthodontic literature, primarily because the benefits have not been consistently reproduced among clinical studies. The present study was a retrospective cephalometric analysis of patients with Class II, division 1 malocclusions at the start of treatment. There were 51 children treated by a single clinician with a MARA in combination with Edgewise appliances. The comparison group consisted of subjects treated with fixed Edgewise appliances only from the University of Tennessee, Department of Orthodontics. Subjects in the two treatment groups were matched on a one-to-one basis for sex and for five cephalometric variables (SNA, SNB, ANB, NAP, FMA) to ensure comparability. The key question was whether the cephalometric results at the end of treatment were comparable between the two groups. The MARA subjects were treated on an average of 2.8 years compared to the Edgewise group with a treatment time of 2.5 years. The Edgewise group had a larger proportion of premolars extracted for treatment compared to the MARA subjects, $69 \%$ and $4 \%$, respectively. Cephalometrically, ANB was on average $1^{\circ}$ smaller in the MARA group. Overall, the lower incisors proclined in both treatment groups during treatment. Similar skeletodental endpoints were achieved in both groups regardless of treatment mechanics. We conclude that either treatment are viable treatment options in the treatment of Class II division 1 malocclusions.
\end{abstract}




\section{TABLE OF CONTENTS}

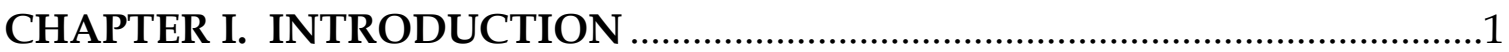

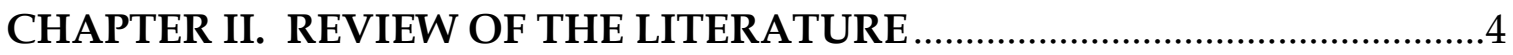

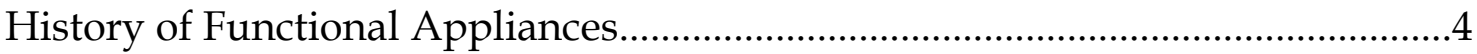

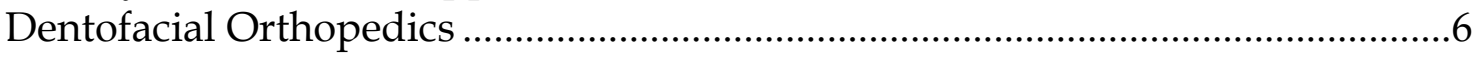

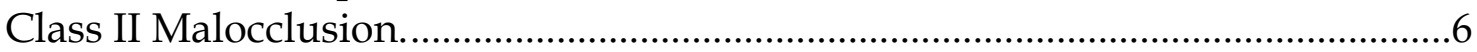

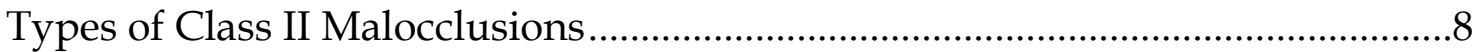

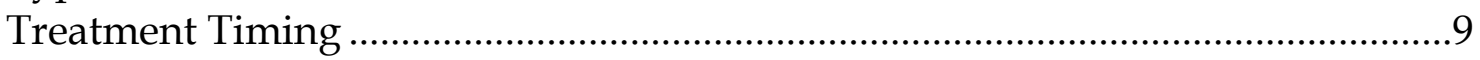

Modifying the Growth Pattern of the Class II Patients ..........................................10

Effects of the MARA on Class II Malocclusions ........................................................10

Effects of the MARA on the Position of the Lower Anterior Teeth.......................12

Outcomes of Functional Appliances .....................................................................13

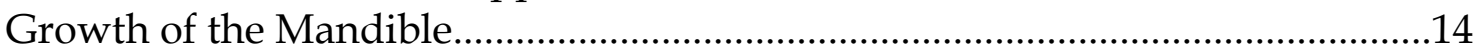

Basic Remodeling Principles .............................................................................14

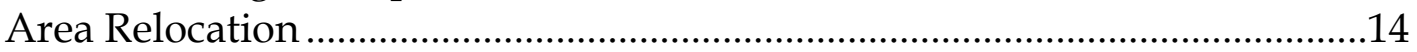

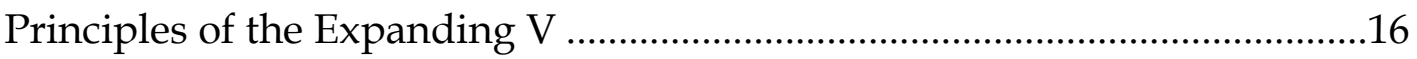

Growth of the Mandible and Sex Differences.......................................................16

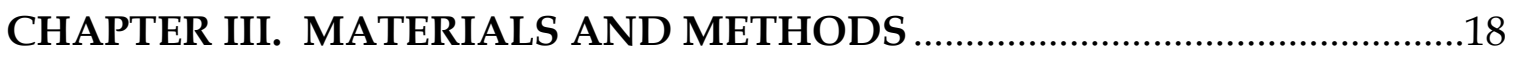

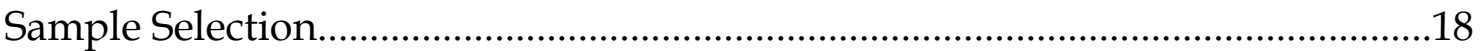

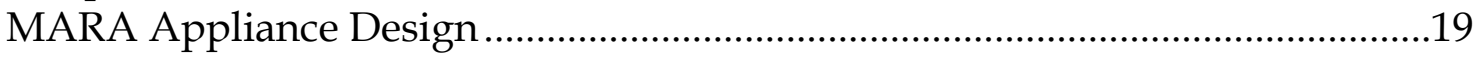

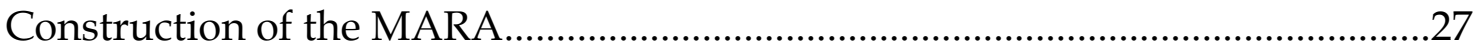

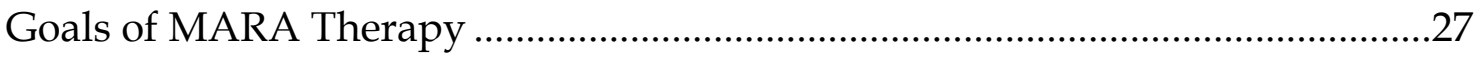

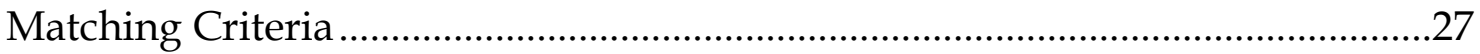

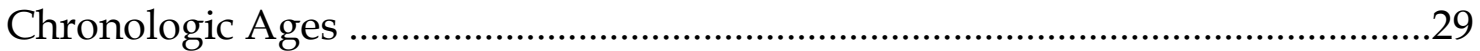

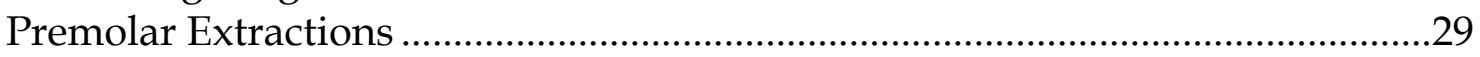

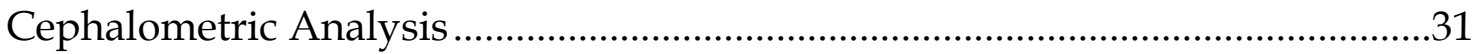

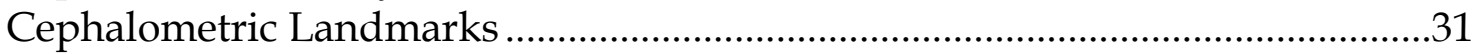

Cephalometric Angular Variables............................................................................33

Cephalometric Linear Variables .............................................................................34

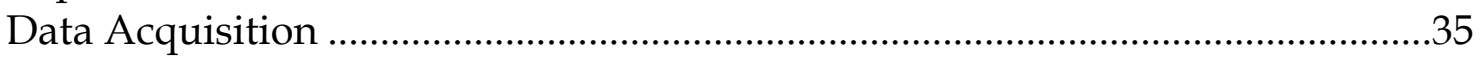

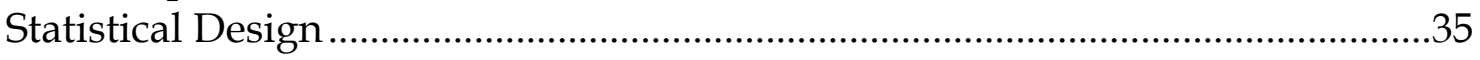

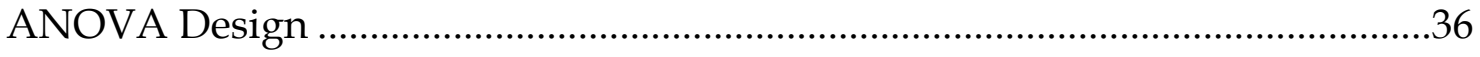

The Reliability of Head Film Measurements ..........................................................36

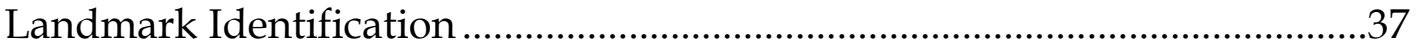

Conventional Angular and Linear Measures ......................................................38

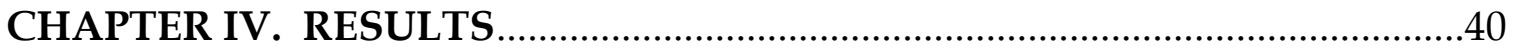

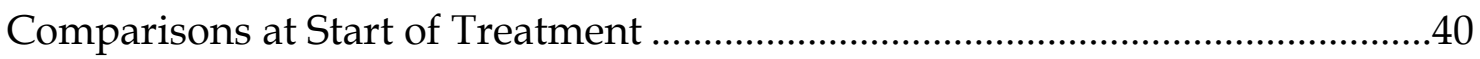

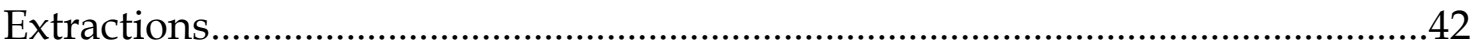




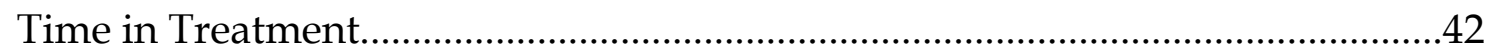

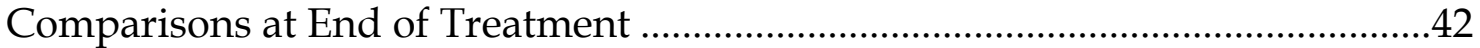

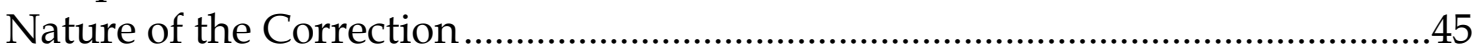

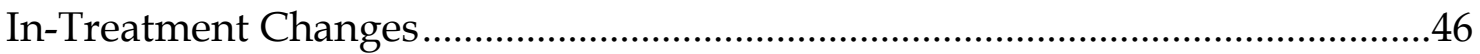

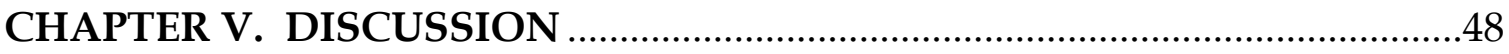

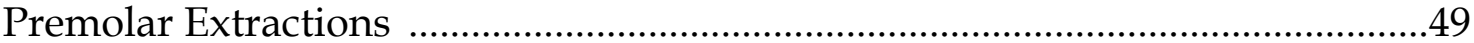

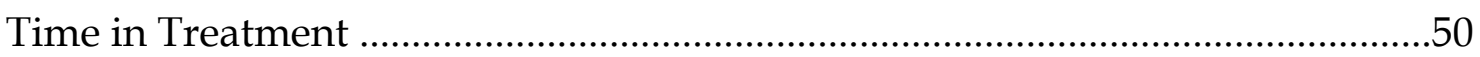

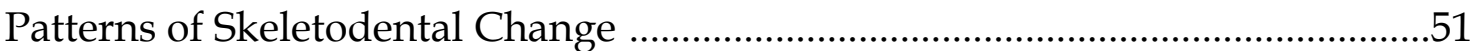

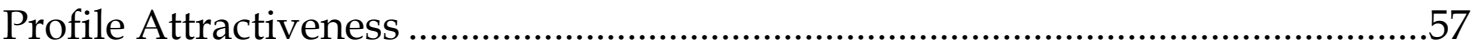

CHAPTER VI. SUMMARY AND CONCLUSIONS …………………….............59

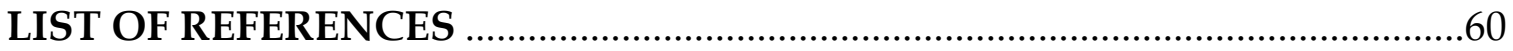

APPENDIX A. SCHEMATIC DIAGRAMS OF LATERAL CEPHALOGRAMS SHOWING THE CONSTRUCTION OF VARIOUS ANGLES AND LINEAR MEASUREMENTS...........................................................67

APPENDIX B. DESCRIPTIVE STATISTICS, BY TREATMENT AND SEX, FOR THE CEPHALOMETRIC VARIABLES EVALUATED AT THE START OF TREATMENT.

APPENDIX C. DESCRIPTIVE STATISTICS, BY TREATMENT AND SEX, FOR THE CEPHALOMETRIC VARIABLES EVALUATED AT THE END OF TREATMENT

APPENDIX D. DESCRIPTIVE STATISTICS, BY TREATMENT AND SEX, FOR THE IN-TREATMENT CHANGE IN CEPHALOMETRIC VARIABLES

APPENDIX E. MEAN IN-TREATMENT CHANGES, BY TREATMENT AND SEX, OF THE CEPHALOMETRIC VARIABLES

APPENDIX F. CEPHALOMETRIC TRACINGS 


\section{LIST OF TABLES}

Table 1. Descriptive statistics for ages of the patients in the two, matched treatment groups ...................................................................................

Table 2. Results of mixed-model analysis of variance tests to evaluate the comparability of the matched samples at the start of treatment.........41

Table 3. Results of mixed-model analysis of variance tests to evaluate the comparability of the matched samples at the end of treatment..........44

Table 4. Results of mixed-model analysis of variance tests to evaluate the comparability of the matched samples for the in-treatment changes 


\section{LIST OF FIGURES}

Figure 1. A schematic diagram of the mandible showing the various areas of relocation

Figure 2. Frontal view of the MARA ……………............................................20

Figure 3. Maxillary occlusal view of MARA ………………………………......21

Figure 4. Mandibular occlusal view of MARA with the lower lingual arch .....22

Figure 5. Left lateral view of the MARA …………………..................................23

Figure 6. Close-up of left lateral view of MARA ………………………………...24

Figure 7. Schematized buccal view and description of MARA ...........................25

Figure 8. Schematized frontal view and description of MARA...........................26

Figure 9. Example of the profile enhancement seen on a patient once the MARA is cemented in place................................................................28

Figure 10. Cephalometric diagram showing the location of the skeletodental landmarks used in this study .32

Figure 11. Means of 5 variables, by treatment, at the end of treatment .52

Figure 12. Mean in-treatment changes in A Point to Nasion-perpendicular, by treatment and by sex

Figure 13. Mean in-treatment changes in B Point to Nasion-perpendicular, by treatment and by sex. .55 


\section{CHAPTER I. INTRODUCTION}

Class II malocclusions are common, with approximately one-third of United States youths having this parasagittal malrelationship (Brunelle et al. 1996; Cozza et al. 2006). In a typical orthodontic practice, the correction of Class II malocclusions may constitute almost half of the cases (Franchi and Baccetti 2001). A Class II malocclusion stems from diverse etiological factors. A common feature in this malocclusion is insufficient mandibular length rather than an excess in the size of the maxilla (Graber et al. 2005; Moyers et al. 1980). There are several ways for the specialist to treat this sort of skeletal discrepancy.

The treatment modalities to correct a Class II malocclusion include an array of extra-oral appliances, arch expansion appliances, various extraction patterns, functional jaw orthopedic appliances, and, most intrusively, orthognathic surgery to advance the mandible (McNamara and Brudon 1993). Each treatment modality "differs in its effect on the skeletal structures of the craniofacial region, sometimes accelerating or limiting the growth of the various structures involved" (McNamara and Brudon 1993).

A conventional form of fixed orthodontic treatment is extra-oral anchorage that is achieved using a facebow or headgear alone or with full appliances in the hope of restraining maxillary growth in combination with favorable growth of the mandible (Graber et al. 2005). The low pull or cervical face-bow is used with patients exhibiting short lower facial heights. The facebow appliance has two bows, an inner and an outer bow. The inner bow is anchored to molar tubes on the bands of the upper first molars, and the outer bow is attached to a strap extending around the cervical region and anchored by the back of the neck. Headgears attach directly to the arch wires placed in full fixed appliances using J-hooks. The force of the headgear will retract flared maxillary incisors and restrict growth of the maxilla (Graber et al. 2005).

Another treatment modality to correct a Class II molar relationship is to distalize the maxillary molars in a patient with an orthognathic profile. This can be achieved by using various distalizing plates, pendulum appliances, or nickel titanium coils (Franchi and Baccetti 2001). One type of distalizing plate is the acrylic cervical occipital appliance (ACCO), which consists of an "acrylic palatal section, modified Adams clasps to the first premolars, and finger springs against the mesial surfaces of the maxillary first molars" (Franchi and Baccetti 2001). The appliance fits against the maxillary teeth creating a force against the first molars that results in posterior movement of the first molar. This appliance is worn all of the time except during meals, and it is usually accompanied by a cervical or highpull face-bow during the night (Franchi and Baccetti 2001). 
The pendulum appliance was first described by Hilgers in 1992. It consists of an acrylic button in the palate to serve as anchorage and titanium molybdenum alloy (TMA) springs to deliver a distal force to the maxillary molars. Once the molars have been over-corrected to a super Class I occlusion, Hilgers recommends leaving the appliance in place for 6 to 10 weeks to prevent mesial drift of the molars (Franchi and Baccetti 2001). Using this approach to treatment, Bussick and McNamara (2000) found a small increase in lower anterior facial height and a slight opening of the Frankfort Mandibular Plane angle (FMA). Alternatively, nickel titanium coils placed in front of the maxillary molars can effectively distalize molars. This can be done successfully once anchorage concerns have been considered (Franchi and Baccetti 2001).

There are other alternatives to the approaches discussed above to treat a Class II malocclusion. Functional appliances provide yet another treatment option for a growing child with a retrognathic profile. These appliances can be used effectively in the mixed or early permanent dentition. Functional appliances have been used to position the mandible forward with the intent of enhancing mandibular growth. According to Ward et al. (2005), the forward positioning of the condyle enhances metabolic action in the temporomandibular joint (TMJ). Graber et al. state, "increased anabolic and catabolic exchange, not merely forward positioning, may account for enhanced growth of the condyle and posterior wall proliferation" (2005). The treatment of skeletal malocclusions can be facilitated if facial growth can be anticipated (Dudas and Sassouni 1973).

Another treatment approach to Class II correction is the extraction pattern of maxillary and mandibular premolars. Either, maxillary first premolars and mandibular second premolars can be extracted or under some circumstances maxillary first premolars and mandibular first premolars can be extracted. Lastly, in severe cases, orthognathic surgery can advance the mandible to resolve the mandibular deficiency.

The mandibular anterior repositioning appliance (MARA) was developed in 1991 by Douglass Toll of Germany. Jim Eckhart of California had been using the Herbst with successful treatment outcomes, but his patients complained of lip and cheek irritation, so Eckhart and Toll began experimenting to improve the MARA. In 1995, with the help of Ormco and AOA laboratory, a new design was unveiled for clinical use (Allen-Noble 2002).

The MARA is a tooth-borne functional appliance (Pangrazio-Kulbersh et al. 2003). The intended effects of the MARA are to enhance mandibular growth, thereby correcting the Class II skeletal malocclusion. The clinical consequences of the MARA have not been tested to any extent. 
The purpose of the present study is to add information through the analysis of cephalometric radiographs about the skeletodental effects of the MARA used in conjunction with conventional Edgewise treatment.

Sequentially-treated orthodontic cases were studied in which MARA therapy was used in conjunction with full fixed appliances. These outcomes were compared to matched cases treated with only full fixed Edgewise appliances in the early permanent dentition. The goal of this cephalometric study is to identify any skeletal benefits obtained from the additional use of the MARA in the treatment of skeletal Class II subjects. 


\section{CHAPTER II. REVIEW OF THE LITERATURE}

\section{$\underline{\text { History of Functional Appliances }}$}

The use of functional appliances dates back to 1879 when Norman Kingsley used a removable appliance to "jump the bite" of an excessively retrognathic patient. Kingsley's bite jumping appliance is considered to be the prototype of functional appliances (Wahl 2006). Functional appliances can either be fixed, such as the Herbst, MARA, and Jasper Jumper, or removable, such as the Bionator and Fränkel; they can then be further subdivided into tissue borne or tooth borne appliance designs.

Edward $\mathrm{H}$. Angle employed an appliance that is comparable to the MARA. It consisted of "a pair of interlocking rings, soldered to opposing first molar bands" to position the mandible forward (Wahl 2006). Another appliance that is comparable to the MARA is the Oliver guide plane (Wahl 2006). Pierre Robin invented a removable vulcanite monobloc that served as a removable mandibular positioning device. The vulcanite monobloc was used in infants exhibiting severe retrognathia (notably in what is now termed the Pierre Robin sequence) to prevent blockage of the airway with the tongue (Graber et al. 2005). Viggo Andresen added a lingual flange of acrylic on his appliance that forced the mandible forward 3 to 4 millimeters. He placed this appliance in his daughter before she left for her summer vacation and when she returned, the appliance, worn only at night, had corrected her mandibular retrognathia. Andresen subsequently applied this technique to other patients to correct their Class II malocclusion that he could not treat with his conventional fixed appliance (Wahl 2006).

The Herbst appliance was developed in the early 1900's by Emil Herbst (McNamara and Brudon 1993), but findings on the effectiveness of this appliance were not published until 1935 (Wahl 2006) with Hans Pancherz being credited with renewing the interest in the Herbst appliance during the 1970s (Gönner et al. 2007). The Herbst appliance is appropriate for children in the early permanent dentition whom might not be compliant. The Herbst appliance positions the mandible forward with a "telescoping mechanism that is connected to the first molars at one end and a cantilevered arm attached to the mandibular molars at the other end" (Wahl 2006).

Rolf Fränkel (1908-2001) invented an appliance that corrects malocclusions with minimal contact with the teeth. The Fränkel appliances are complex, and the oral vestibule is the operational basis for the treatment with these appliances. 
Fränkel developed three appliances, FR-1, FR-2, and FR-3, that correct Class I, Class II and Class III malocclusions, respectively (Fränkel R, Fränkel C 1989).

Wilhelm Roux, in 1883, was among the first in dentistry to study the influences of natural forces and functional stimulation on form. Roux's studies laid the groundwork for "functional dental orthopedic principles" (Wahl 2006). Alfred P. Rogers, the father of myofunctional therapy, was the "first to implicate the facial muscles for the growth, development, and form of the stomatognathic system" (Wahl 2006).

The use of functional appliances became prevalent in the United States in the middle 1970s as a result of the outcome of altered functional position of the mandible in a series of primate (rhesus monkeys) studies. Studies on both rats and monkeys have concluded facial sutures react to their mechanical environment, forward positioning of the mandible in the rat does not increase the mitotic activity of the cells in the condyle to be of any clinical significance and forward positioning of the mandible in the primate does initiate the remodeling activity with the temporomandibular joint which can thus alter the direction of condylar growth (Meikle 2007). The last conclusion drawn from Meikle's experiment has the most clinical significance to the orthodontist especially in the treatment of the actively growing child. Although the use of primates is on the decline, rats continue to be widely studied examining the adaptation of the temporomandibular joint to the protrusive positioning of the mandible (Meikle 2007).

Varying appliances were constructed that altered the functional position of the mandible, and the adaptations were studied cephalometrically and histologically. Skeletal adaptation can also occur in the direction and extent of growth of the head of the mandibular condyle (McNamara 1973). Studies subsequently conducted on humans resulted in less than impressive findings when compared to the earlier animal studies (Chen et al. 2002). Even today, the efficacy of functional appliances to correct Class II skeletal malocclusions is controversial with no significant differences being found with the functional appliances (Harrison et al. 2009). It is difficult to find definitive answers about the efficacy of functional appliances because there are many inconsistencies in measuring treatment outcomes. For example, some investigators use Condylion (Cd) as the posterior end point in measuring the overall mandibular length, whereas others use Articulare (Ar) (Chen et al. 2002). According to Chen and coworkers, randomized controlled trials and discriminant analysis show that functional appliances have a minute clinical effect on mandibular length. Dolce et al. (2007) list three possibilities for mandibular growth achieved with a functional appliance: "increased beyond its genetic potential; accelerated when there is an increase in the growth rate during treatment, followed by a period of 
slow growth, thereby achieving the expected growth; or anterior mandibular positioning with adaptation as further growth occurs".

Certain criteria are proposed for case selection when functional appliances are desired for treatment of a Class II patient. Barton et al. state that these should be "a well-aligned lower arch, a well-aligned upper arch, a Class I [or] mild Class II skeletal pattern, forward posture of the mandible by the patient will give a satisfactory soft tissue profile, and a person who is undergoing active growth" (1997). These conditions are said to be the foundation for undergraduate teaching in the United Kingdom (Barton et al. 1997). The treatment phase using a functional appliance aims to decrease overjet and correct any discrepant molar relationship.

\section{Dentofacial Orthopedics}

The year 1985 was a conceptual turning point for the specialty of orthodontics. Thomas M. Graber, then editor of The American Journal of Orthodontics, changed the name of the orthodontic journal to The American Journal of Orthodontics and Dentofacial Orthopedics (AJO-DO) after approval of the trustees and House of Delegates of the American Association of Orthodontics (AAO). The change was modest and went unnoticed by many in the specialty, but it helped lay the groundwork for the future of orthodontics. The AAO, in 1994, officially made the name change of the specialty from orthodontics to orthodontics and dentofacial orthopedics. The American Dental Association (ADA) approved the name change during the same year (Hamilton 1997). Orthodontists are the best qualified practitioners to provide facial orthopedic therapy due to their extensive education and experience with facial growth; conversely, failure on an orthodontist's part to provide dentofacial orthopedics can inhibit the treatment potential of a patient presenting with severe facial dysplasia (Hamilton 1997). In The United States, dentofacial orthopedics is a relatively quiescent term and has received little positive recognition or acceptance (Hamilton 1997).

\section{Class II Malocclusion}

The National Health and Nutrition Examination Survey (NHANES III), was conducted by the National Center for Health Statistics between 1988 and 1994. Orthodontic researchers felt there was a need to assess the magnitude of various occlusal problems in the general population (Brunelle et al. 1996). One component recorded in this study was overjet (to the nearest whole millimeter). $15 \%$ of the U.S. population has an overjet greater than $5 \mathrm{~mm}$, which reflects a 
Class II skeletal relationship, whereas an additional $38 \%$ has an overjet in the range of 3 to $4 \mathrm{~mm}$. About $8 \%$ of the population had an overjet in excess of $6 \mathrm{~mm}$ or more. Younger children between the ages of 8-11 have the most severe overjet $(3.4 \mathrm{~mm}$ ), whereas older children and adults have an overjet of $3.0 \mathrm{~mm}$ (Brunelle et al. 1996). Among American whites, African Americans and Mexican Americans, Mexican Americans had less overjet than the African Americans (Brunelle et al. 1996).

The Class II malocclusion presents in the primary dentition as a distal step of the second molars. These children have an excessive overjet, a Class II molar relationship, and a Class II canine relationship. It is noteworthy that children with Class II malocclusions commonly have a deficiency of the mandible that is maintained after the permanent first molars erupt (Baccetti et al. 1997). Children exhibiting a distal step in the deciduous dentition typically progress into either an end on Class II or a full step Class II malocclusion in the permanent dentition (Bishara et al. 1988). Children with a distal terminal molar relationship had a shorter mandibular corpus length with a larger gonial angle compared with children with Class I occlusion. Baccetti et al. concluded that children exhibiting a Class II malocclusion in the deciduous dentition will never self correct to a Class I occlusion in the absence of treatment (1997). Treatment should be initiated in children as soon as the orthodontist deems the patient is ready to begin (Baccetti et al. 1997).

Baume (1950 a, b, c) studied casts of 30 children taken at annual intervals to determine the developmental changes in the deciduous dental arch. Baume found that once the deciduous dental arches were formed the sagittal and transverse dimensions did not change "except when subjected to inadequate environmental influences" (Baume 1950:131). In a later study by Baume (1950 b), he studied 60 casts to describe the three kinds of molar adjustment that occur both before and after the eruption of the permanent molars. When the deciduous dentition terminated with a definitive mesial step, the permanent first molars subsequently erupted into Class I interdigitation without disturbing the position of the neighboring teeth (Baume 1950). When the primary dentition is spaced with a straight terminal plane, he observed that after the eruption of the permanent first molars the straight terminal plane developed into a mesial step. This occurred due to closure of the lower primate space (Baume 1950). The primary dentition with no spacing forces the permanent molars to erupt into an end to end occlusion. Class I occlusion was obtained by a late mesial shift of the mandibular permanent first molars once the deciduous second molars exfoliated (Baume 1950). 


\section{Types of Class II Malocclusions}

Class II malocclusions can loosely be subdivided into Class II division 1 and Class II division 2. Class II division 1 malocclusions present with an orthognathic maxilla, retrognathic mandible, and protrusive maxillary incisors. Class II divisions 2 malocclusions present with orthognathic maxilla, retrognathic mandible, and retroclined maxillary incisors (Feldmann et al. 1999).

Moyers et al. (1980) studied 697 lateral cephalograms of North American white children who had either undergone treatment for an Angle Class II malocclusion by an orthodontist or were a part of an untreated control group. The study found some general characteristics of Class II malocclusions. Many of their linear measurements are smaller than the mean values in the normative data base (as determined from the Michigan Growth Study; Riolo et al. 1976) and, as a rule, Class II patients have smaller faces. Six subgroups (Types A, B, C, D, and $\mathrm{E}$ ) based on horizontal variables, were identified and among them four (Types B, C, D, and E) are true syndromic types with different skeletal and dental features (Moyers et al. 1980).

Type A is characterized as having a normal skeletal pattern and normal occlusal plane. The mandibular dentition is in a normative position on its base with the maxillary dentition located in a protracted relationship, thus causing the Class II molar relationship with a larger than normal overjet and overbite (Moyers et al. 1980). Type B has a Class II skeletal pattern with a prognathic maxilla and a mandible in a normal relationship anteroposteriorly. Type $C$ has a severe Class II skeletal pattern with a short mandible and maxilla. The lower incisors are tipped labially with the upper incisors either upright or labially inclined.

Type $\mathrm{D}$ has a retrognathic skeletal profile due to a small mandible and a maxilla that is normal or slightly diminished. The mandibular incisors are either lingually inclined or upright and the maxillary incisors are labially inclined. Type E displays a severe Class II profile because of a prognathic midface and a normal mandible. Maxillary and mandibular dentitions tend to be positioned forward of their base with the incisors being inclined labially. Type F has the mildest Class II tendency and can be considered as a milder nonsyndromal form of Type B, C, D, or E. This large subgroup is not well defined but displays some skeletal Class II characteristics with the mandible and midface being small. In Types C, D, and F extraoral traction to the maxilla is avoided and the main focus is placed on enhancing mandibular growth.

Moyers et al. (1980) identified five vertical subgroups of the Class II malocclusion (Types 1 through 5), but these vertical types are not as clearly 
differentiated as the horizontal types discussed previously. Type 1 has a higher than normal mandibular plane angle, a steep functional occlusal plane and the palate is tipped downward. The anterior facial height is greater than the posterior facial height, leaving these patients to be referred to as "long face syndrome" by oral surgeons and "high angles" by orthodontists. Type 2 has a mandibular plane, functional occlusal plane and palatal plane that is flatter than normal. The incisors tend to be upright with excessive overbite. Type 3 is characterized by an upward and anteriorly positioned palatal plane resulting in a tendency towards open bite. If the mandibular plane angle is higher than normal a skeletal open bite is inevitable.

Type 4, on the other hand, has a mandibular plane, functional occlusal plane, and palatal plane that are tipped significantly downward resulting in a gummy appearance. The maxillary incisors are tipped labially and the mandibular incisors are tipped lingually. This subgroup is the rarest and most severe of the vertical types. Type 5 is similar to Type 2 with a normal mandibular and functional occlusal plane. The palatal plane is tipped downward resulting in a skeletal deep bite. The mandibular incisors are labially inclined and the maxillary incisors are nearly upright.

In a study conducted by McNamara (1981), lateral cephalometrics were reviewed of 277 Class II children with an average age of 9.0 years and the following results were obtained. The position of the maxilla is normal in the majority of the Class II individuals. If the position of the maxilla is abnormal, it tends to be retrusive rather than protrusive. When a child exhibits a long anterior lower facial height with a steep Frankfort Mandibular Plane Angle (FMA), both the maxilla and mandible are in a retrusive position in relation to the cranial base structures. Almost half of Class II individuals have normally positioned upper incisors, but approximately $30 \%$ are retruded and $60 \%$ of these patients exhibited mandibular skeletal retrusion (McNamara 1981).

\section{$\underline{\text { Treatment Timing }}$}

The ideal timing of treatment for pre-pubertal children presenting with Class II malocclusions is controversial. Proponents of early intervention argue that it is "outrageous" to allow abnormal function to continue, leading to worsening malocclusion (Arvystas 1998). Advocates of early treatment also cite enhanced cooperation in the pre-adolescent child, decreased need for extraction to correct molar discrepancy, decreased chance of trauma of the proclined incisors, and psychological benefits (King et al. 1999). Early treatment can, with adequate growth, correct the molar relationships and achieve skeletal facial harmony (Arvystas 1998). An important factor to consider concerning optimal 
timing of treatment in children is the uncertain growth potential (Proffit and Tulloch 2002). Orthodontists can estimate the growth potential by looking at one or more measures of physiological age, but it should be understood that mandibular growth may be unpredictable and may show random variation in the timing and the amount of growth (Mitani and Sato 1992).

\section{Modifying the Growth Pattern of Class II Patients}

The aim of treating Class II individuals with mandibular retrognathia is to enhance the growth of the condyle by positioning the mandible anteriorly utilizing dentofacial orthopedics. In a randomized clinical trial by Tulloch et al. (1998), the investigators addressed whether "the growth pattern of Class II patients can be modified" and "if differential growth effects can be induced during early treatment [do] these effects in the end make any difference" (1998). In Tulloch's study, three groups were formed by randomization "equivalent with regard to age, sex, maturity, and morphologic measures from the cephalograms and study models" (Tulloch et al. 1998). The results showed that early treatment with functional appliances was less efficient because it did not reduce the average time the patient was in treatment with fixed appliances during the second phase of treatment. Also, this extra phase of treatment did not reduce the incidence of extractions nor orthognathic surgery (Tulloch et al. 1998). On the other hand, researchers at the University of Florida found that patients who had undergone phase one treatment completed phase two approximately six months faster than patients treated in one phase (Wheeler et al. 2006). When total treatment time is considered, patients undergoing two phase treatment were treated for a significantly longer period (Wheeler et al. 2006). Wheeler et al. showed that, by the end of phase two, the discrepancy was indistinguishable between the patients receiving phase one treatment and the patients who did not (2006). In the end, Proffit and Tulloch point out that the above findings should not negate the importance of early treatment for some children. Each patient should be examined individually as to the benefits of early treatment depending on psychological issues, whether they are accident prone, and their level of skeletal maturation and whether their skeletal maturation is ahead of their development dentally (Proffit and Tulloch 2002).

\section{Effects of the MARA on Class II Malocclusions}

At present, the only published article on the effects of the MARA is a study by Pangrazio-Kulbersh et al. (2003) who investigated the MARA's skeletal and dental effects on anterior, posterior and vertical changes in 30 Class II patients. Twelve boys with a mean age of 11.2 years and 18 girls with a mean 
age of 11.3 years were treated with the MARA for an average of 10.7 months. Lateral cephalometric radiographs were taken 2 weeks prior to treatment and 6 weeks after the MARA was removed. The patients treated with the MARA were compared to 21 untreated Class II control subjects, of which there were 13 girls and 8 boys. The control subjects were from the Michigan Elementary and Secondary School Growth Study and longitudinal cephalograms were available for the age ranges studied. The MARA patients were also compared to Fränkel and Herbst groups that had been reported previously (Pangrazio-Kulbersh et al. 2003).

The results show that the MARA produced detectable treatment effects on the skeletal and dental components of the craniofacial complexes. The MARA group showed that on average $1.1 \mathrm{~mm}$ of distal movement of the maxillary molar, whereas the control group showed the molar migrated mesially an average of $1.3 \mathrm{~mm}$. The MARA tends to restrict the eruption of the maxillary first molar. Inferior movement of the maxillary first molar was only $0.1 \mathrm{~mm}$ per year in the experimental group, whereas the control group showed $0.9 \mathrm{~mm}$ of inferior movement. The MARA group showed forward movement of 1.2 and 0.6 $\mathrm{mm}$ for the mandibular molar and incisor, respectively, while the changes were only 0.5 and $-0.4 \mathrm{~mm}$ movement, respectively, for the control group. IMPA increased $3.9^{\circ}$ annually for the experimental group but only increased $0.3^{\circ}$ for the control group. Mandibular length, as measured from Condylion to Gnathion, increased annually $4.8 \mathrm{~mm}$ in the experimental group but only $2.1 \mathrm{~mm}$ in the control group. Chin point, as measured from Pogonion to Nasion-perpendicular, moved anterior by an average of $2.3 \mathrm{~mm}$ in the experimental group but just 0.3 $\mathrm{mm}$ in the control group. ANB decreased, on average, $-1.4 \mathrm{~mm}$ annually in the experimental group, but only $-0.1 \mathrm{~mm}$, on average, in the control group. Mandibular anterior face height increased $2.5 \mathrm{~mm}$ annually on average in the experimental group, but only $1.0 \mathrm{~mm}$ in the control group. As for posterior facial height, it increased $4.0 \mathrm{~mm}$ in the MARA group and the control group only increased $1.3 \mathrm{~mm}$. There was no change in the mandibular plane angle during treatment with the MARA (Pangrazio-Kulbersh et al. 2003).

According to Pangrazio-Kulbersh et al., there is no maxillary skeletal effect in patients treated with the MARA, but there is movement of the maxillary molar $-1.1 \mathrm{~mm}$ in a posterior direction (2003). The distalizing effect of the MARA on the maxillary molars is a large part of the dental correction of the Class II malocclusion. When the mandible is hyperpropulsed forward, its length increased an average of $2.7 \mathrm{~mm}, \mathrm{SNB}$ decreased $1.0^{\circ}$, ANB decreased $1.3^{\circ}$ due to the forward movement of B Point, and the chin moved anteriorly $2.0 \mathrm{~mm}$. The lower anterior face height (ANS-Me) of patients treated with the MARA increased approximately $2.5 \mathrm{~mm}$ per year, and the posterior face height (Cd-Go) also increased, approximately $4.0 \mathrm{~mm}$ per year. According to the authors, the 
increase in posterior face height could be attributed to the stainless steel crowns on molars causing the condyle to be positioned in a more inferior position in the glenoid fossa, thereby stimulating growth of the condyle in a more superiorposterior direction. These skeletal changes indicate that the MARA can be effective in treating Class II skeletal discrepancies by increasing the mandibular length, and this appliance has no headgear effect on the maxilla (PangrazioKulbersh et al. 2003).

Effects of the MARA on the Position of the Lower Anterior Teeth

In a retrospective study conducted by Gönner et al. (2007), the effects of the MARA on the lower incisors were studied. The study consisted of four age groups: group one consisted of 36 children under the age of 12, group two consisted of 28 adolescents between 13 and 18 years of age, group three was the combination of groups one and two for a total of 64 subjects, and group four consisted of 26 adults between the ages of 19 to 56 years. This study did not distinguish between males and females. Cases were treated with a combination of the MARA and multi-bracket system with at least a two-by-four appliance on the upper and/or lower teeth. Lateral cephalograms were taken in habitual occlusion before the onset of treatment (T1), when the MARA was removed (T2), and one year after the appliance was removed (T3). All cephalograms were evaluated by one of the authors using Onyx Ceph 2.7.18 (Gönner et al. 2007).

The study found that the lower anterior teeth moved forward by an average of $0.4 \mathrm{~mm}$ between T1 and T2 in group 1, and they moved posteriorly 0.2 $\mathrm{mm}$ between $\mathrm{T} 2$ and T3. The anterior teeth moved $1.0 \mathrm{~mm}$ in group 2 between $\mathrm{T} 1$ and $\mathrm{T} 2$ and moved posteriorly $0.7 \mathrm{~mm}$ between $\mathrm{T} 2$ and T3. There was an anterior movement of the lower anterior teeth of $0.7 \mathrm{~mm}$ between T1 and T2 in group 3 and posterior movement of $0.5 \mathrm{~mm}$ between T2 and T3. In group 4 there was $1.7 \mathrm{~mm}$ of anterior movement in the lower anterior teeth and posterior movement of $0.2 \mathrm{~mm}$ between $\mathrm{T} 2$ and T3. In all four groups, the average reduction in overjet was between $4.0 \mathrm{~mm}$ and $5.0 \mathrm{~mm}$. There is an association between the more lingually inclined lower incisors and the greater the anterior movement of the lower incisors. That is, the smaller the IMPA, the more mesial incisor movement is expected (Gönner et al. 2007).

The author suggested that there is a neuromuscular reprogramming process that occurs through a biofeedback mechanism affecting the masticatory system. The lower molars show more bodily movement than tipping movement since the MARA appliance is situated near the molar's center of resistance. The force is parallel to the occlusal plane in an anterior direction and is transmitted to a larger proportion of the periodontal ligament (PDL). Since the force is largely 
on the molars, the authors suggest that the supporting bone is mainly cortical, which provides the best anchorage. This study shows that the MARA is effective in correcting dental and skeletal Class II malocclusions in various age groups. However, in the adult patient there are greater dentoalveolar effects on the lower anterior teeth than is seen in the children (Gönner et al. 2007). The study did not consider the effect of multi-bracket therapy on the lower incisors when worn concurrently with the MARA appliance.

\section{$\underline{\text { Outcomes of Functional Appliances }}$}

In a study by Franchi and Baccetti (2006), they found a range of results using functional appliances to achieve functional jaw orthopedics. When treatment is carried out at the peak of mandibular growth as detected by a reliable indicator of skeletal maturity, "good responders" to Class II treatment with functional jaw orthopedics showed a "biannual increase in Co-Gn greater than $5.3 \mathrm{~mm}$ ", whereas "bad responders" to Class II treatment with functional jaw orthopedics were defined as "subjects showing a biannual increase in Co-Gn equal to or smaller than $5.3 \mathrm{~mm}^{\prime \prime}$ (2006:952). Franchi and Baccetti found that the Condylion-Gonion-Menton angle provides a reliable discriminator between good and bad Class II responders to functional jaw orthopedics (2006). When this angle is smaller than $125.5^{\circ}$, there is a greater response to the functional jaw orthopedic appliance (Franchi and Baccetti 2006). The growth rate of the Class II jaw to functional orthopedics is greater when there is anterior growth rotation of the mandible instead of posterior rotation of the mandible (Franchi and Baccetti 2006). The spatial relationship of the mandible to the craniofacial structures is not a significant predictor of the direction of mandibular growth, nor of patient responsiveness to these appliances (Franchi and Baccetti 2006). However, change in the inclination of the condyle to the mandibular base is closely related to the growth features during pubertal growth spurt of the mandible. When the functional jaw orthopedic is used on a Class II patient during puberty, alteration in condylar growth direction is the main skeletal effect (Franchi and Baccetti 2006).

According to Graber et al. (2005), sagittal modification is a combination of increases in condylar growth, condylar growth in a more favorable direction, and adaptation and repositioning of the articular eminence and fossa. Additionally, some functional appliances restrain the maxillary dentoalveolar area. When a functional appliance is worn, the condyle moves into a more forward and downward position on the articular eminence. 


\section{Growth of the Mandible}

The renowned English anatomist, John Hunter, was the first to discover that the mandible grows mostly in the posterior direction and not by elongation at the chin (Harris and Enlow 1964). Hunter was able to show that the ramus grows backward through the addition of new bone onto the posterior border along with some removal from the anterior side of the ramus. A century later, Humphry experimentally confirmed Hunter's concept by inserting small metal rings on the posterior and anterior border of the ramus in a growing pig. The rings on the posterior surface of the ramus became increasingly embedded whereas the rings on the anterior were released from the ramus with continued growth.

Charles (1925) and Brodie (1941) argued that the mandibular condyle is an important site of growth of the mandible that brings about forward and downward growth of the mandible. The rate of growth is proportional to the increase of cortical bone along the posterior border of the ramus and to the growth of alveolar bone on the body of the mandible (Enlow and Harris 1964). According to Harris and Enlow, "structural remodeling is a companion process to growth and functions to maintain the constant shape of the mandible as it continues to increase in size" (1964:29).

In the study by Harris and Enlow (1964), 25 human mandibles were chosen for preparation and microscopic study. Only young, growing mandibles were observed for the study. The microscopic sections were mapped according to the various types of endosteal and periosteal bone deposits throughout all areas of the mandible thus allowing a detailed analysis of growth in each part of the mandible. The findings are reviewed in depth in the following section.

\section{Basic Remodeling Principles}

The mandible grows in many directions at once (Figure 1), which relocates the various parts of the mandible into new relative positions.

\section{Area Relocation}

For example, the condylar neck is relocated into the enlarging ramus as the condyle grows in a superior and posterior direction. The anterior portion of the ramus becomes relocated into the posterior portion of the body as the entire

mandible grows in a posterior direction. As an area of the mandible changes its location, the bone in that area is either partially or totally removed and new 


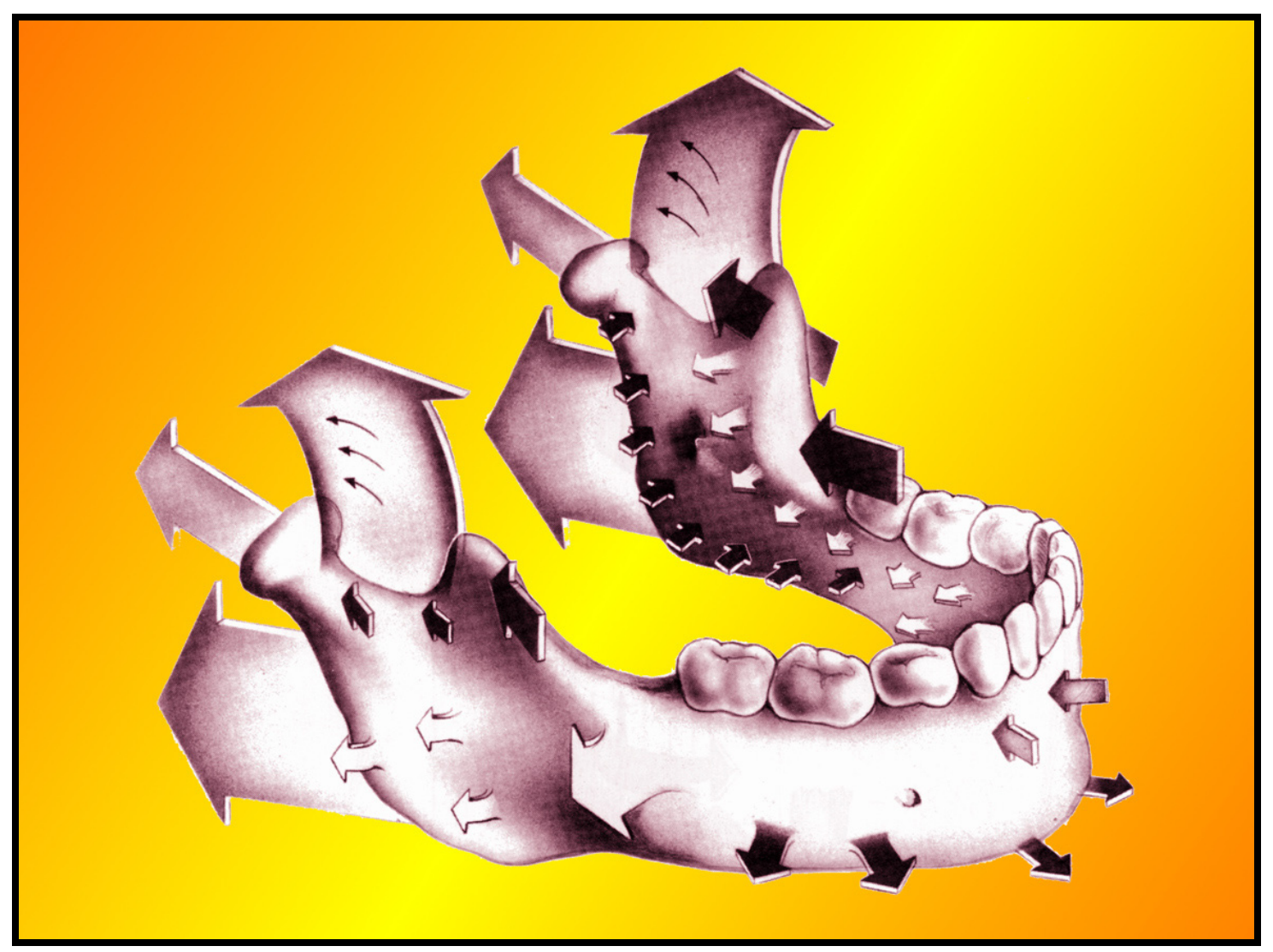

Figure 1. A schematic diagram of the mandible showing the various areas of relocation. Modified by EF Harris with permission from Harris and Enlow. Harris DB, Enlow DH. A study of the postnatal growth of the mandible. Am J Orthod 1964;50:25-50. 
deposits of bone are added onto the old surface. There are two sorts of bone, endosteal and periosteal, which are both produced during growth and remodeling of the mandible. The production of endosteal bone along with resorption of periosteal bone enables the cortex of the mandible to grow and move in a medial direction in response to remodeling that is occurring (Harris and Enlow 1964).

\section{Principle of the Expanding V}

The $\mathrm{V}$ principle is seen frequently in many parts of the growing mandible. There are many parts of the mandible that have the orientation of the cortical plates that form a V. When growth occurs, the V-shaped areas enlarge in their overall shape and size and are moving and growing in a direction that is oriented toward their wide end. The inner surface of the $V$ faces towards the actual direction of growth so the inner surface receives the new deposition of bone. The outer surface of the $\mathrm{V}$ undergoes simultaneous resorption (Harris and Enlow 1964).

Since the mandible grows in a posterior direction, the condyle moves in a posterior and superior direction. Due to area relocation the bone that was in the condylar capitulum will become located in the condylar neck. Growth of the coronoid processs proceeds in three simultaneous directions: superior, posterior and medial. The coronoid process grows mainly by the addition of periosteal bone onto the medial surface with periosteal resorption and endosteal deposition on the buccal surface therefore the base of the coronoid process is being moved in a medial direction, the apex is growing in a buccal direction and the whole process is increasing in size (Harris and Enlow 1964). The body of the mandible is constantly growing into successive areas that were previously occupied by the posteriorly moving ramus. The posterior part of the body increases in length by a continuation of the $\mathrm{V}$ principle.

\section{Growth of the Mandible and Sex Differences}

Maj and Luiz (1964) concluded that the size of the mandible increases (as a percentage) more in females than in males between 9 to 13 years. This difference is due to the increase in height of the ramus being one-third greater in females than in males.

On the other hand, they found that there are no major sex differences in the increase in the length of the mandible (Maj and Luiz 1964). The growth of the mandible occurs in cycles, and the contention of these authors is that there is no 
relation between the incremental growth of the body of the mandible and of the ramus. Additionally, the incremental increase in the mandibular length is not related to the stature of the individual.

According to Lysle Johnston (1998), the amount an individual can be anticipated to grow during treatment might or might not depend on the particular mode of treatment, yet growth is partly a function of age, sex, and time of treatment. Growth curves from any of the large growth studies, i.e. Burlington, Bolton, or Michigan, can provide good estimates of growth intensity. Johnston (1998) studied facial growth from lateral cephalograms of 120 Class II, division 1 patients treated between 1969 and 1980 at St. Louis University as part of a long term comparison between non-extraction and extraction edgewise therapy. Only 12 of the 120 patients studied showed the mandible failing to advance relative to the maxilla during treatment. Six of these 12 were treated by extraction therapy and six were treated by non-extraction therapy. Thirty years of clinical experience led Johnston to conclude that most Class II patients show a favorable growth pattern during treatment (Johnston 1998). 


\section{CHAPTER III. MATERIALS AND METHODS}

\section{$\underline{\text { Sample Selection }}$}

The present study is a retrospective analysis of lateral cephalograms taken on 51 subjects with Class II, division 1 malocclusions at the beginning of their comprehensive orthodontic treatment. These 51 patients received treatment with a MARA followed by or coinciding with full fixed Edgewise appliance treatment. The lateral cephalometric radiographs were available at the beginning of treatment and the end of treatment. These records were acquired from the office of Joe L. Wasson, D.D.S, M.S., Memphis, Tennessee, who treated all of these cases. The control group consisted of a matched sample of 51 subjects with Class II, division 1 malocclusions treated with full fixed Edgewise appliances at The University of Tennessee, Department of Orthodontics in Memphis, Tennessee.

Dr. Wasson uses a three-tier treatment approach for his treatment of Class II malocclusions. The patients who fall within the first tier usually are in the early mixed dentition age, with high mandibular plane angles (FMA), openbite tendencies, and deleterious oral habits (e.g., tongue thrusts, digit habits). These patients usually have excessive overjet with constricted or underdeveloped dental arches due to habits and/or poor lip competence. A patient's treatment typically consists of Fränkel II (FR-2) wear for approximately 22 hours a day for 1 year followed by a retention phase consisting of wearing the appliance for 8 to 12 hours a day. Once all of the permanent teeth have fully erupted, full fixed Edgewise appliances will finish treatment.

The second tier of treatment is aimed at patients exhibiting Class II division 2 malocclusions. These patients have low mandibular plane angles (FMA), deepbite tendencies and a forward growth pattern. These patients are older, 9 to 11 years of age, as compared to the Tier 1 patients but still remain in the mixed dentition. First, the Class II division 2 malocclusion is converted to a Class II division 1. The patient will wear a Bionator full time for 9 to 12 months with 6-to 8-week appointment intervals to assess their progress. Once correction is achieved, the appliance is worn only at night for retention while the rest of the permanent dentition erupts. Next, full fixed Edgewise appliances are used to detail and finish the case.

The third treatment strategy is aimed at Class II patients in the early permanent dentition (11 to 14 years of age). This group of patients is often treated with fixed functional appliances such as the Herbst appliance or MARA (i.e., mandibular advancement repositioning appliance). These patients can have brackets placed on the upper four incisors to aid in aligning the incisors. Alternatively, full brackets can be placed concumently to expedite treatment. 


\section{MARA Appliance Design}

The MARA belongs to the group of permanent intermaxillary functional appliance connections (Gönner et al. 2007). The design of the MARA does not require patient compliance and is a simple treatment solution for the older child with a Class II malocclusion. The typical patient selected for the MARA is still growing, with most of the permanent dentition present, with the maxilla in a favorable position, and the mandible in a retrognathic position. According to Dr. Wasson, even if the patient has ceased growing, the MARA will produce upper and lower dentoalveolar changes. Figures 2 through 6 are views of the MARA.

The basic MARA design (Figure 7) consists of four stainless steel crowns, lower arms soldered to the crowns, archwire tubes soldered to the stainless steel crowns for upper and lower archwires, upper elbow tubes soldered to the crowns, upper elbows shimmed to provide the desired advancement, and lower lingual arch soldered to the lower crowns. Stainless steel crowns are placed on the maxillary and mandibular permanent first molars. Cams are attached at a $90^{\circ}$ angle to the occlusal plane on the upper first molars. Abutments are permanently fixed to the lower first molars to prevent the lower jaw from moving posteriorly beyond a specific position. The upper elbows are removable only by the orthodontist so adjustments can be made either bilaterally or unilaterally to advance of mandible. The lower elbow is permanently attached to either the band or stainless steel crown on the mandibular first permanent molar.

Upper and lower archwire tubes with hooks (Figure 8) are standard features allowing the clinician to use a full fixed appliance concurrently with the MARA. A lower lingual arch (not contacting the lingual surface of the lower anteriors) is recommended to help control tipping of the lower incisors and to prevent the tendency of the upper elbows to rotate and tip the molars mesially and lingually, thus crowding out the lower second premolars. There also is the option to add a habit inhibiting device or rapid maxillary expansion screw to the appliance.

When the patient tries to bite in their habitual Class II position, the fixed lower arms on the MARA interfere with closure, obliging the patient to close only into a Class I relationship. Therefore, the patient is always forced to bite in a more forward position in front of the upper elbow and is guided by the appliance to consistently hold his jaw in a Class I parasagittal relationship. Patients usually adapt to the appliance within two weeks and experience minimal soreness. If further advancement is needed, the cams can be activated by placing shims of differing lengths (Gönner et al. 2007). 


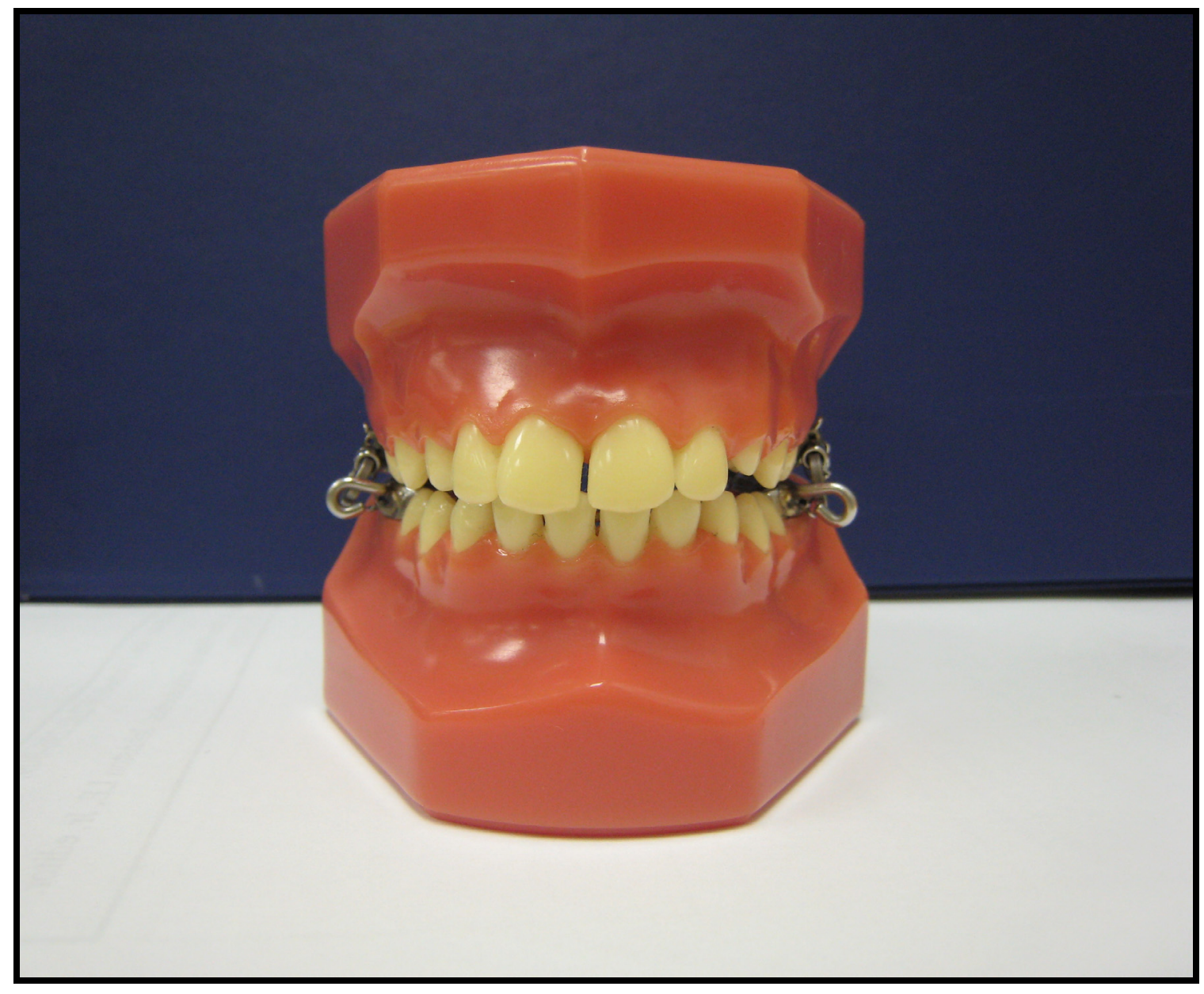

Figure 2. Frontal view of the MARA. 


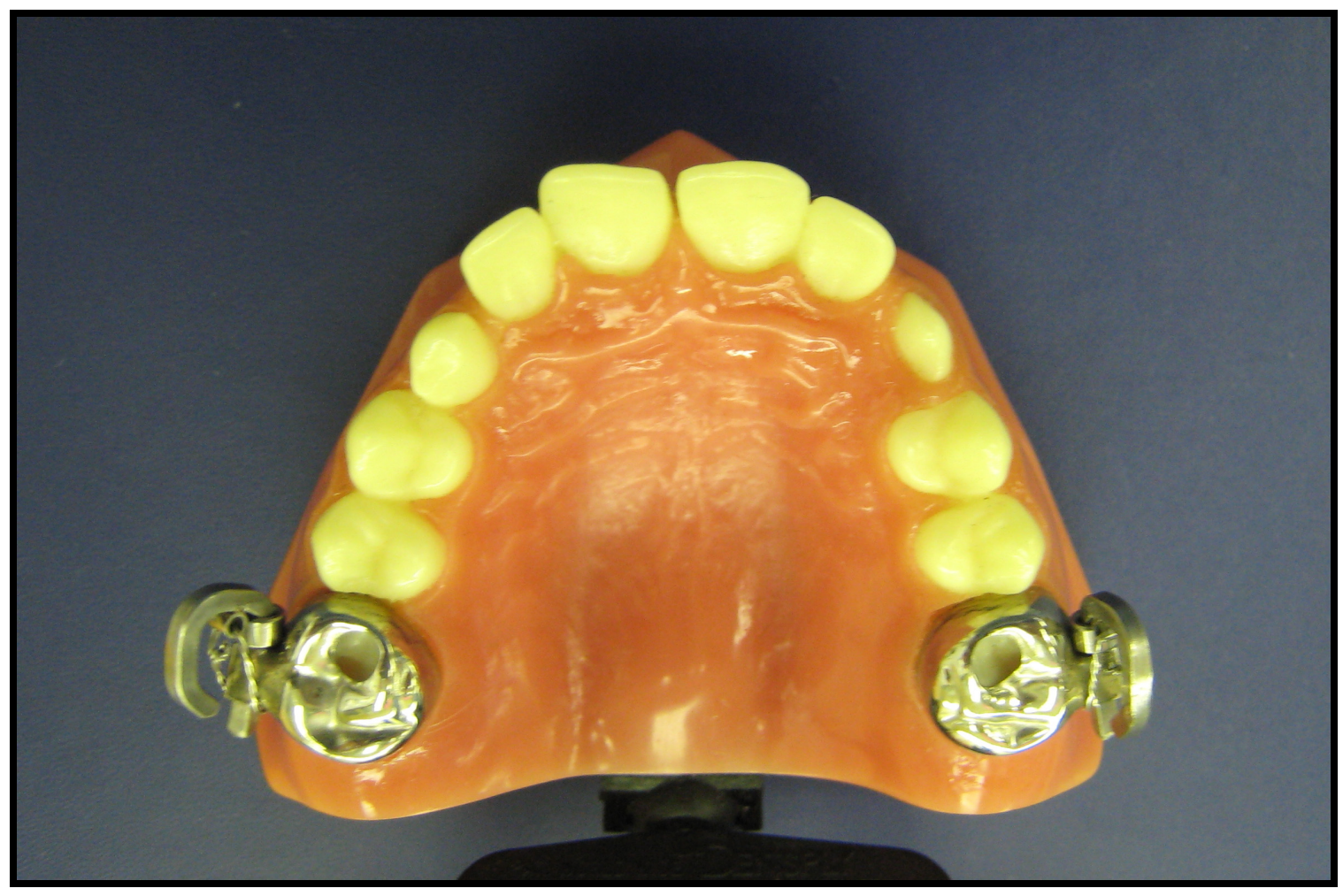

Figure 3. Maxillary occlusal view of MARA. 


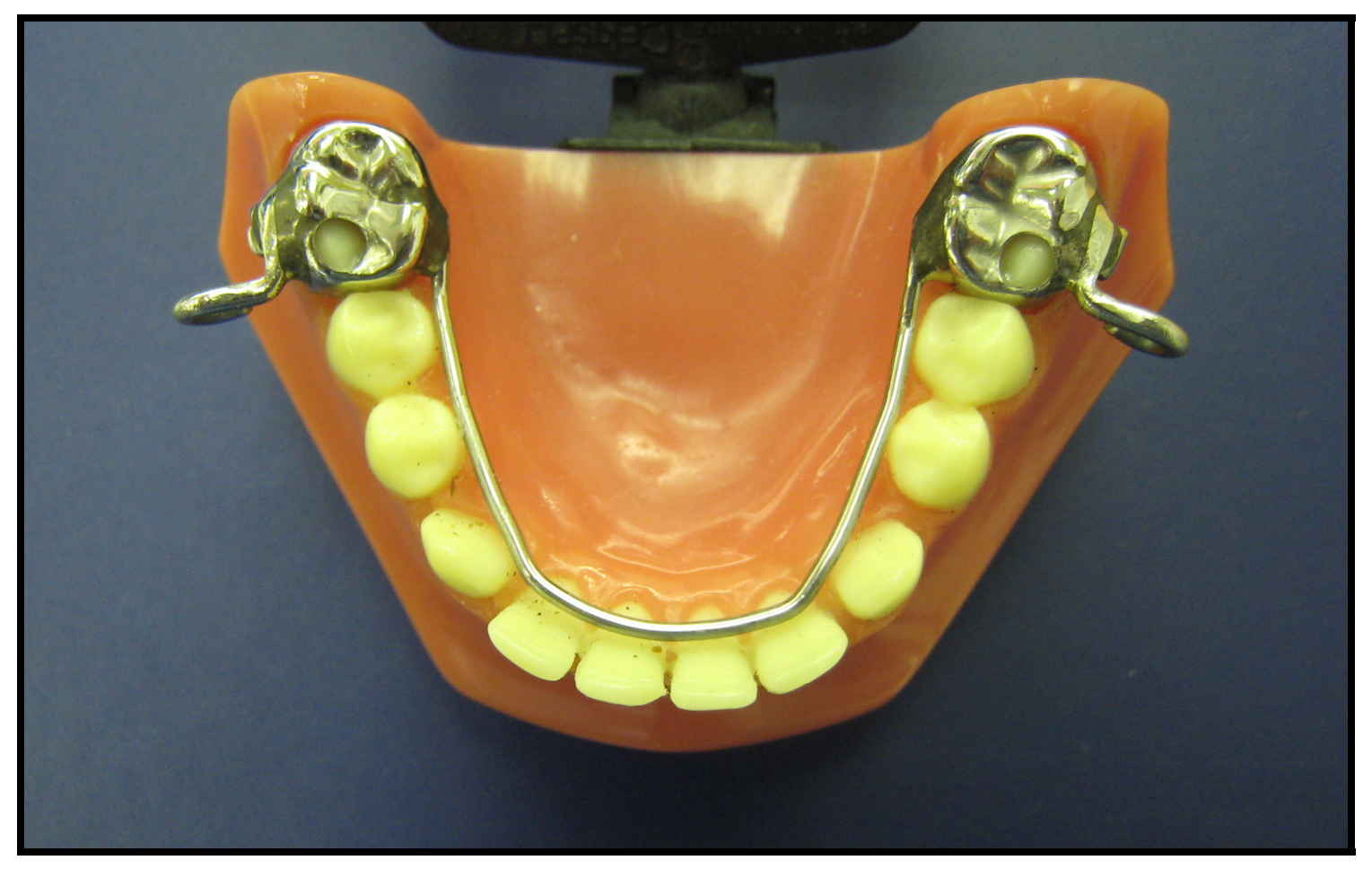

Figure 4. Mandibular occlusal view of the MARA with the lower lingual arch. 


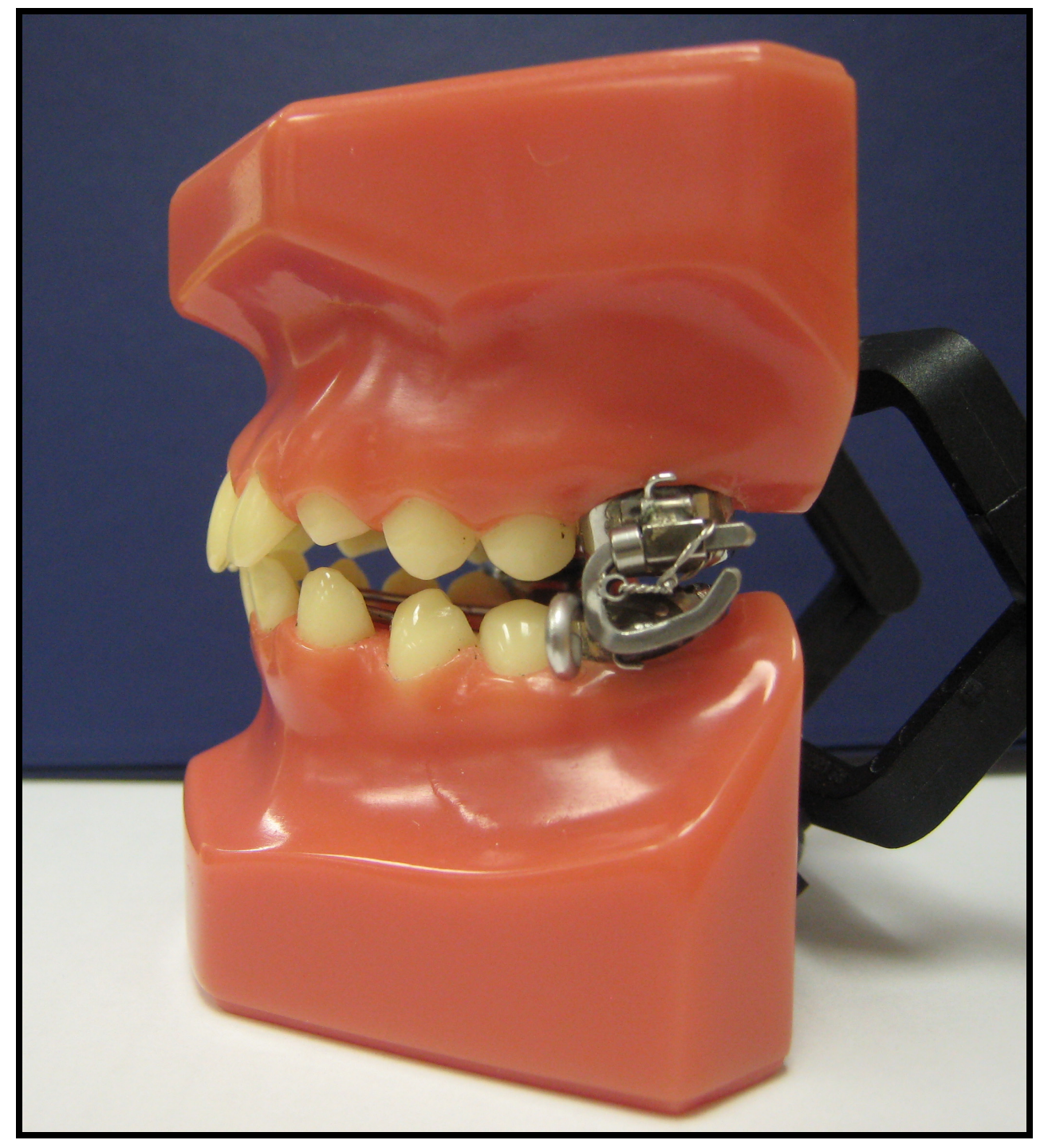

Figure 5. Left lateral view of the MARA. 


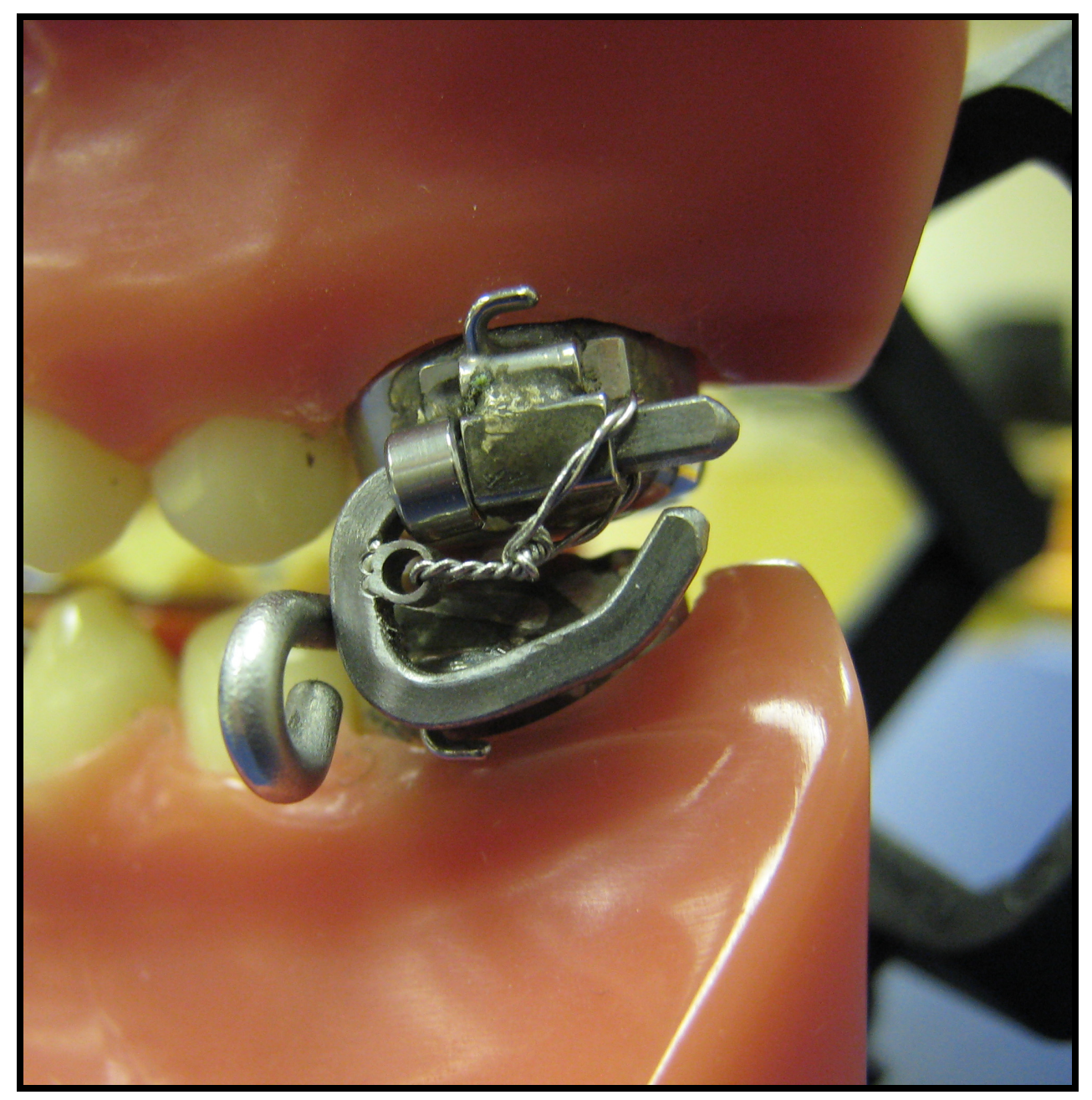

Figure 6. Close-up of left lateral view of MARA. 


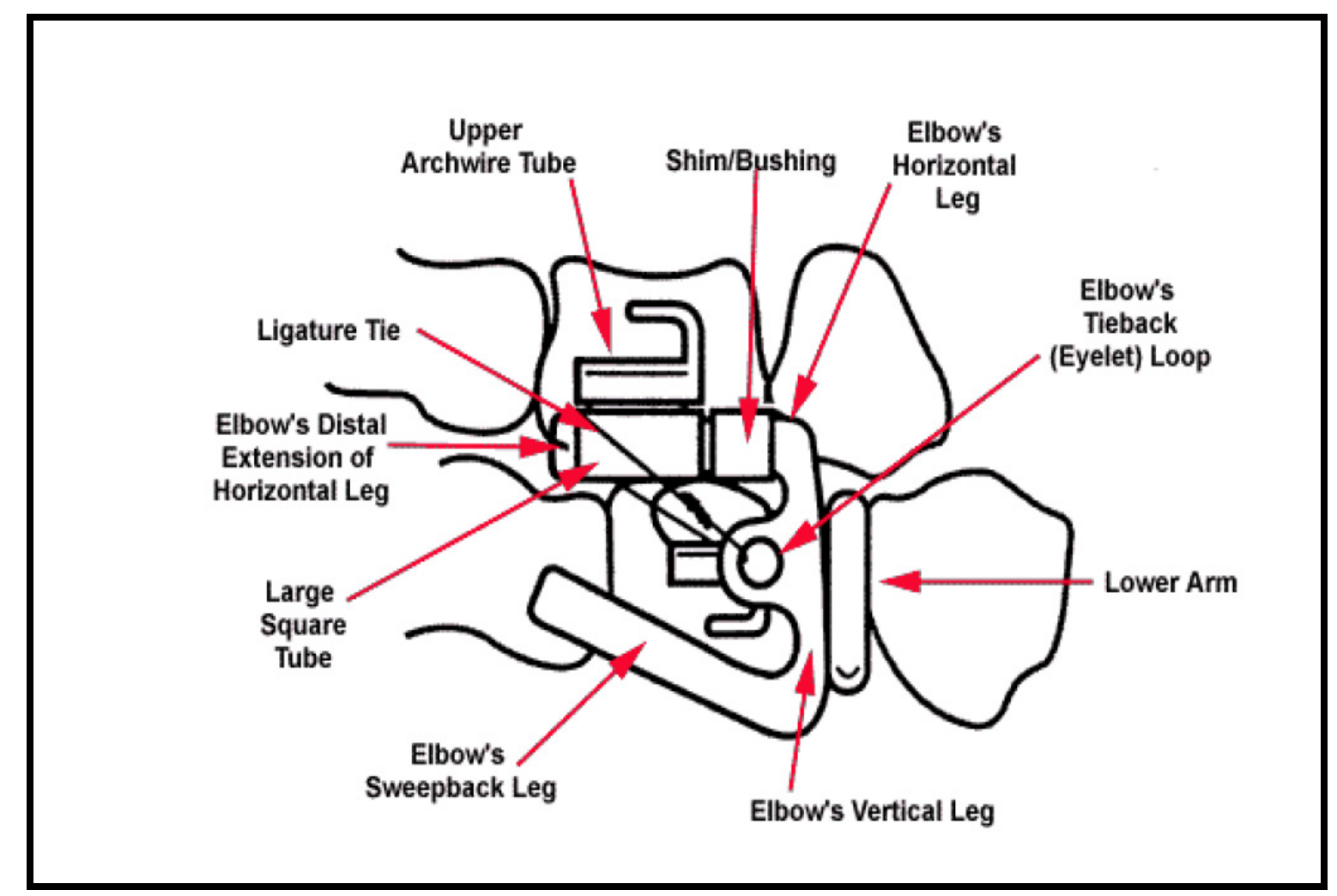

Figure 7. Schematized buccal view and description of MARA. Modified by EF Harris with permission from Allen-Noble PS. Allen-Noble PS. Clinical management of MARA. Allesee Orthodontic Appliances. July 2005. 


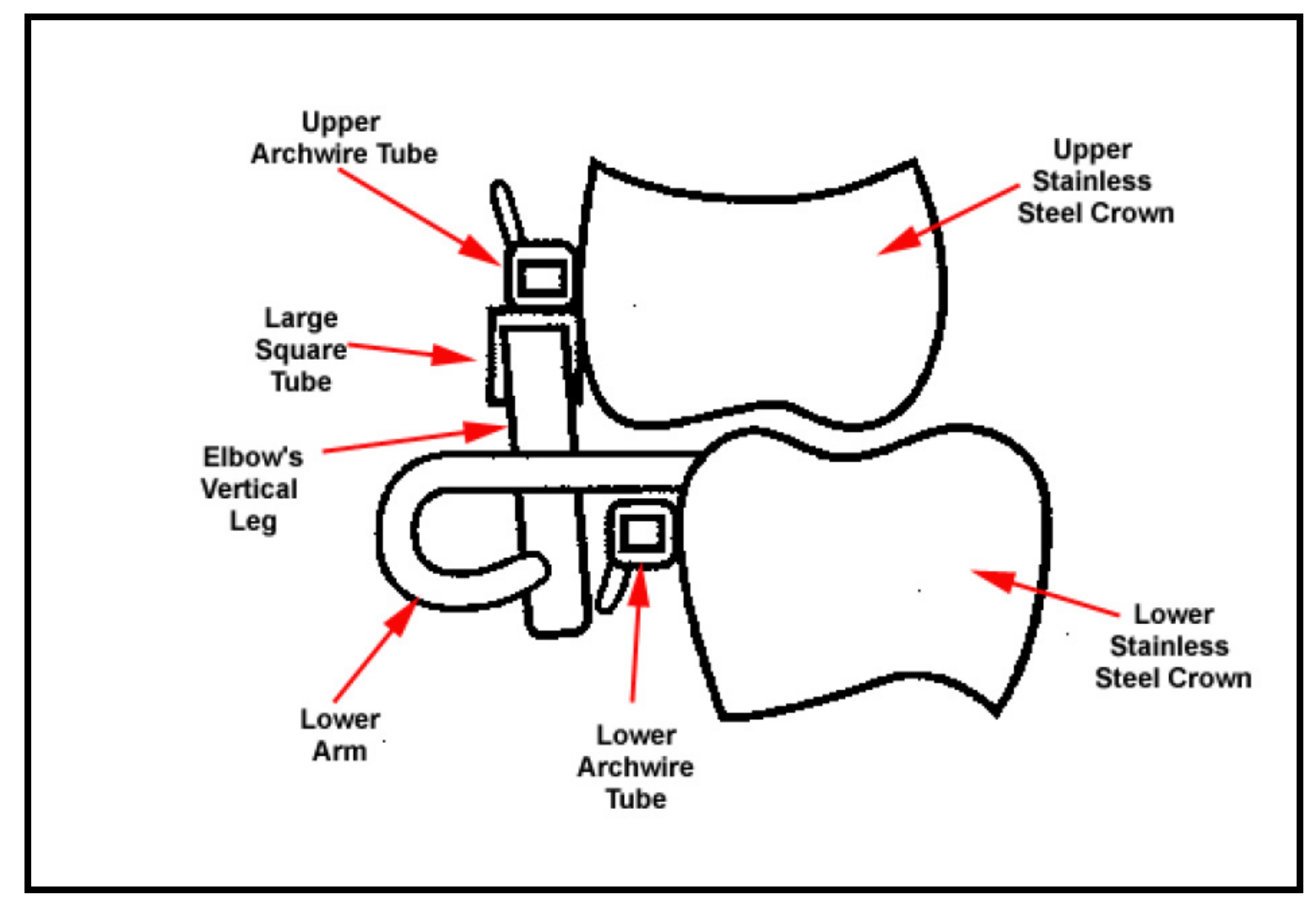

Figure 8. Schematized frontal view and description of MARA. Modified by EF Harris with permission from Allen-Noble PS. Allen-Noble PS. Clinical management of MARA. Allesee Orthodontic Appliances. July 2005. 


\section{Construction of the MARA}

To prepare a patient for a MARA, proper diagnostic records need to be taken, and, obviously, it needs to be deemed that the MARA will be beneficial for that patient. A maxillary and mandibular alginate impression is required free of voids and bubbles, preferably taken in a perforated or metal tray. The casts and prescription sheet will be sent to Allesee Orthodontic Association (AOA) in Wisconsin or the appliance can be made by another laboratory. The bite registration is not necessary when fabricating a MARA. If the overjet is 4 to 5 $\mathrm{mm}$, then advance the MARA to an end-to-end incisor relationship. If the overjet is 8 to $9 \mathrm{~mm}$, then advance the MARA half of this total distance to prevent excessive strain on the temporomandibular joint (TMJ), the musculature, or the appliance, then the remainder of the advancement can be made over the next several months. Separators need to be placed one to two weeks prior to cementation of the MARA.

\section{Goals of MARA Therapy}

Once the MARA is in place, the patient's profile is immediately enhanced due to the mandible being positioned forward hyperpropulsed. Consequently, in terms of differential diagnosis, the patient's maxilla needs to be in a favorable position with only the mandible lacking sufficient length. The MARA will distalize the upper molars, which is desirable when the patient exhibits a Class II molar relationship. Since the MARA is cemented in place, patient compliance is not a factor. Unlike the Herbst appliance, the MARA is more esthetic and can remain in place during the first year of retention to maintain sagittal correction of the Class II malocclusion (Graber et al. 2005). Expectations are that, over the course of treatment of about 12 months, the MARA reshapes the condyle, remodels the fossa, rotates the temporal bone, inhibits maxillary growth, condylar growth, and dentoalveolar movement (Allen-Noble 2005). Figure 9 is an example of the profile enhancement seen on a patient once the MARA is cemented in place.

\section{Matching Criteria}

The main focus of the present research is to evaluate the outcomes of treatment between two samples: (1) patients treated in the early permanent dentition with a MARA coincident or followed by full fixed Edgewise appliances and (2) patients treated conventionally using full fixed Edgewise appliances in the early permanent dentition. 


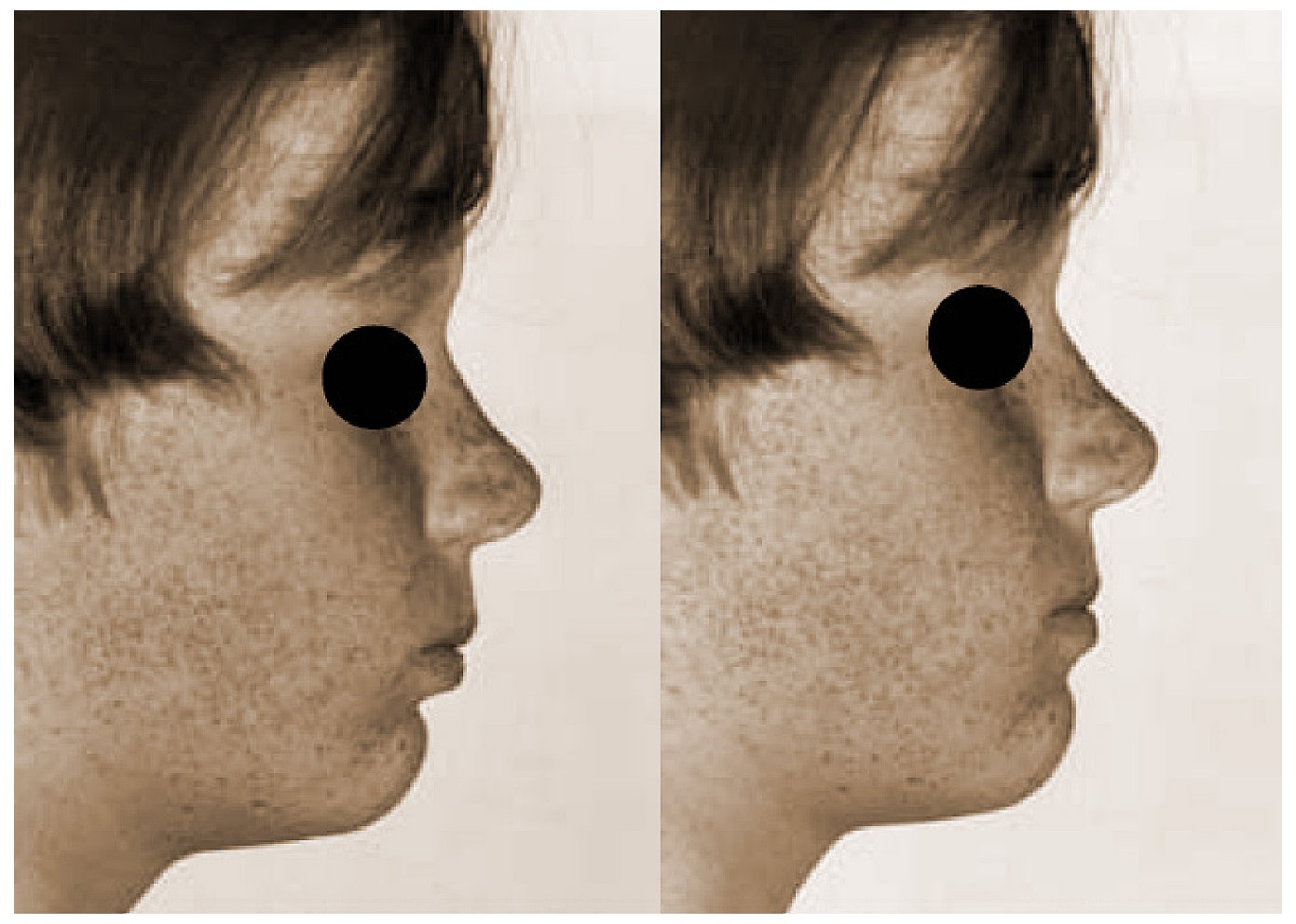

Figure 9. Example of the profile enhancement seen on a patient once the MARA is cemented in place. (Left) Patient with a typical Class II profile and (Right) patient with lower jaw positioned forward to an incisor edge-to edge relationship using the MARA. Modified by EF Harris with permission from Allen-Noble PS. Allen-Noble PS. Clinical management of MARA. Allesee Orthodontic Appliances. July 2005. 
MARA cases $(n=51)$ followed by fixed full appliance were matched caseby-case to 51 conventional fixed Edgewise appliance cases based on five key cephalometric criteria, namely Sella-Nasion to Point A angle (SNA), Sella-Nasion to Point $B$ angle (SNB), ANB angle, A Point to Nasion to Pogonion angle (NAP), and the Frankfort-Mandibular Plane angle (FMA). The average age at the beginning of MARA treatment is 12.6 years, and the average age at the beginning of fixed Edgewise appliance in the sample is 13.1 years.

\section{Chronologic Ages}

Amounts of facial growth depend on the patient's age, so it is important to match the ages between the MARA-Edgewise and the Edgewise-only groups at the start of treatment. Matching was done - as with the cephalometric variables - at the start of treatment while also matching for the patient's sex.

Matching was successful (Table 1). Average age at the start of treatment was about 13 years of age in both groups $(\mathrm{P}=0.11)$. Also, while not matched, results show that the two groups also are comparable at the end of treatment, at about $15 \frac{1}{2}$ years $(P=0.52)$.

With comparable starting and ending ages, it is predictable that the duration of treatment are the same statistically $(\mathrm{P}=0.07)$. Average time in treatment was 2.7 years for the total sample. At face value, treatment time is a lot longer in the MARA series (2.8 vs. 2.5 years), but, again, this difference of threeto-four months is not significant statistically. Also, this difference may represent differences in operators rather than the appliances. Also, the bulk of Edgewiseonly cases were treated with premolar extractions, which tends to shorten treatment times.

\section{Premolar Extractions}

A substantive difference in the two treatment groups is the frequency of premolar extractions- and premolars were the only teeth removed for orthodontic treatment. Just 2 of the 51 cases $(4 \%)$ were treated with extraction, and this contrasts against the majority of cases $(69 \%$; 35/51) treated with extractions in the Edgewise-only group. 
Table 1. Descriptive statistics for ages of the patients in the two, matched treatment groups. ${ }^{1}$

\begin{tabular}{|c|c|c|c|c|c|c|c|c|c|}
\hline \multirow[b]{2}{*}{ Time } & \multicolumn{3}{|c|}{ MARA-Edgewise } & \multicolumn{3}{|c|}{ Edgewise-Alone } & \multicolumn{3}{|c|}{ Paired t-Tests } \\
\hline & $\overline{\bar{x}}$ & $\mathrm{sd}$ & $\overline{\text { sem }}$ & $\overline{\mathrm{x}}$ & sd & sem & t-test & $\mathrm{df}$ & p-value \\
\hline Start Age & 12.58 & 1.08 & 0.15 & 13.14 & 2.07 & 0.29 & 1.63 & 50 & 0.1090 \\
\hline End Age & 15.41 & 1.05 & 0.15 & 15.62 & 1.95 & 0.27 & 0.65 & 50 & 0.5175 \\
\hline TX Duration & 2.83 & 0.90 & 0.13 & 2.48 & 0.96 & 0.13 & -1.85 & 50 & 0.0699 \\
\hline
\end{tabular}

1Statistics are based on 51 paired sets of patients. 


\section{Cephalometric Analysis}

All cephalometric radiographs in this study were evaluated in norma lateralis, so Frankfort Horizontal is oriented horizontally. Two lateral cephalograms were obtained from each case, also at the beginning of the treatment and at the end of full appliance treatment. The Edgewise group, treated with full fixed appliance, also has two lateral cephalograms, namely from the beginning of treatment and the end of treatment. The following is a list, in alphabetical order, of definitions of the cephalometric landmarks used in this study (Figure 10). All dental landmarks are located on the permanent teeth. The schematic diagrams of the lateral cephalograms showing the construction of various angles and linear measurements can be found in Appendix A.

\section{Cephalometric Landmarks}

A Point (Subspinale): the deepest midline point on the ventral maxillary border inferior to the anterior nasal spine (ANS) and superior to Prosthion (Downs 1948).

ANS Anterior Nasal Spine: the spinous process of the maxilla forming the most anterior projection of the floor of the nasal cavity.

Ar Articulare: the intersection of the radiographic shadow of the interior surface of the cranial base and the posterior surfaces of the columns of the mandibular condyles.

B Point (Supramentale): the deepest midline point on the bony curvature of the mandible between Infradentale and Pogonion (Downs 1948).

Ba Basion: the most dorsal point on the anterior rim of the foramen magnum, which corresponds to the anterior limit of the foramen magnum.

$\mathrm{Cd}$ Condylion: the most superior-posterior point on the curvature of the capitulum of the condylar process.

Gn Gnathion (anatomic): the most anterior-inferior point on the exterior surface of the mandibular symphysis.

Go Gonion (anatomic): the most posterior-inferior point on the gonial process of the mandible.

LIE Incision Inferius: the incisal tip of the most anterior mandibular central incisor.

UIE Incision Superius: the incisal tip of the most anterior maxillary central incisor.

L1A Lower Incisor Apex: the apex of the mandibular central incisor.

L6C L6 Cusp: the mesial cusp tip of the mandibular first molar.

Me Menton: the most inferior point on the exterior symphyseal outline as seen in the lateral film (Athanasiou 1995). 


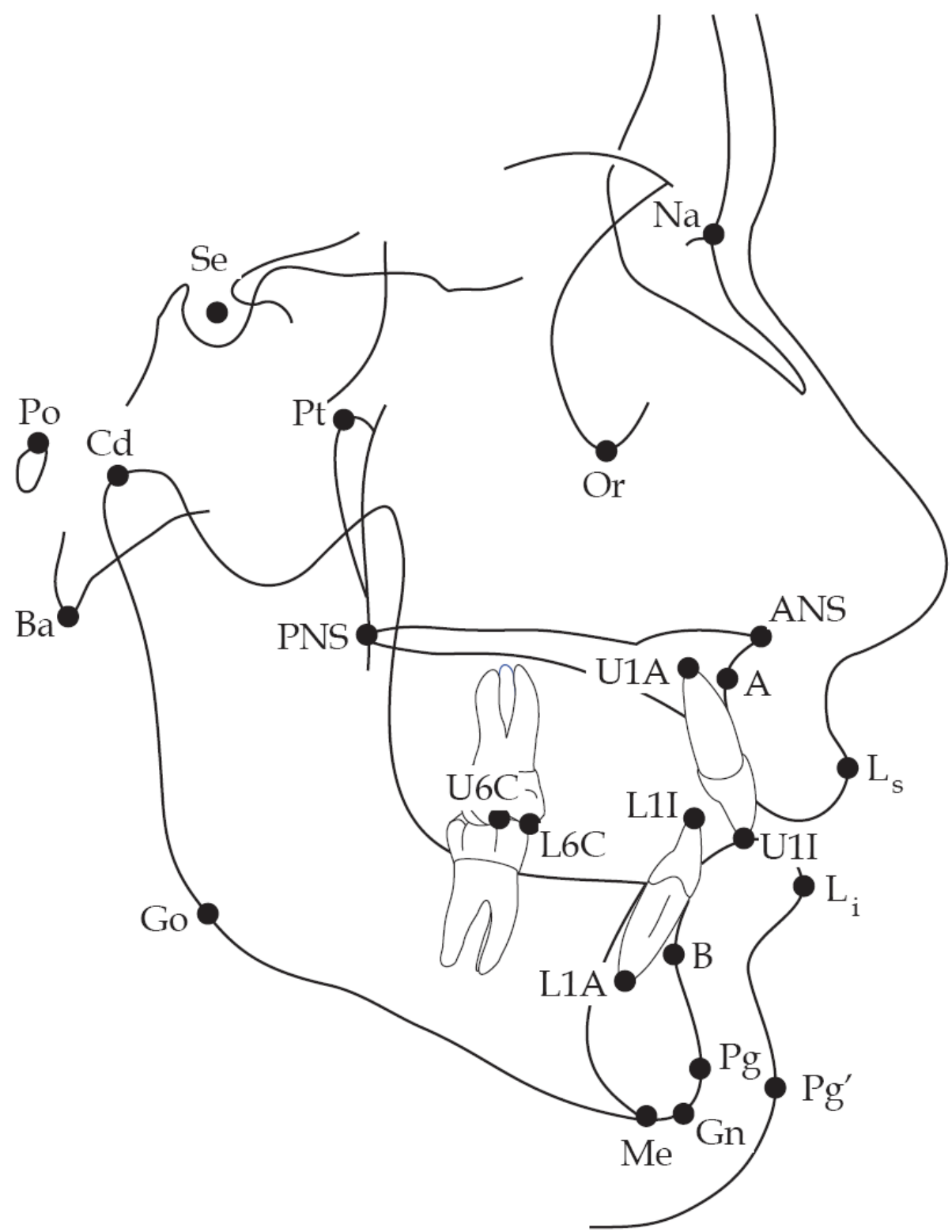

Figure 10. Cephalometric diagram showing the location of the skeletodental landmarks used in this study. All skeletal and dental landmarks were identified with the image orientation in norma lateralis. 
$\mathrm{Na}$ Nasion: the junction of the frontal and nasal bones at the most dorsal point (Athanasiou 1995).

Or Orbitale: the lowest point on the inferior margin of the bony orbit (Athanasiou 1995).

Pg Pogonion: the most anterior point on the anterior contour of the bony chin.

PNS Posterior Nasal Spine: the most posterior point at the midsagittal plane on the bony hard palate.

Po Porion: the most superior point along the superior rim of the external auditory meatus. Anatomic Porion was used, not a mechanical construct (Athanasiou 1995).

$\mathrm{Pt} \quad$ Pterygomaxillary Fissure: the most superior-posterior point on the radiographic outlines of the pterygomaxillary fissure.

Se Sella Turcica: midpoint of the hypophyseal fossa (sella turcica), constructed by inspection (Athanasiou 1995).

UIA Apex of the Maxillary Central Incisor: the apical end of the most anterior maxillary central incisor of the same tooth used to locate $\mathrm{I}_{\mathrm{s}}$.

U6C U6 cusp: the mesiobuccal cusp tip of the maxillary first molar.

\section{Cephalometric Angular Variables}

ANB: the difference between the SNA and SNB angles (Jacobson 1975:126).

$\mathrm{FH}$ to Na-Pg (Facial angle): the inside- inferior angle formed by the intersection of the Frankfort Horizontal and the facial plane, which is defined by Nasion and Pogonion (Athanasiou 1995:255).

FMA (Frankfort-Mandibular Plane Angle): the inferior-anterior angle formed by the intersection of Frankfort horizontal plane (Orbitale-Porion) and the mandibular plane (Gonion-Menton) (Tweed 1969).

FMIA (Frankfort-Mandibular Incisor Angle): the superior-posterior angle formed at the intersection between the line Porion-Orbitale and the line lower incisor edge-lower incisor apex (Baumrind and Frantz 1971:510).

IMPA: the superior-posterior angle determined by lower incisor edge, at the intersection between the line lower incisor edge-lower incisor apex, and the line Gonion-Menton and the point midgonion (Baumrind and Frantz 1971:509).

L1 to NB: the angle formed by the intersection of the line Nasion-Point B and the line lower incisor apex-lower incisor edge (Baumrind and Frantz 1971:510).

Na-Point A-Pogonion (the Angle of Convexity): the superior angle. If A Point is posterior to the line $\mathrm{Na}-\mathrm{Pg}$, the angle of convexity carries a negative sign) (Baumrind and Frantz 1971:509). 
Sella-Nasion-Point A (SNA): the posterior-inferior angle formed by the intersection of the two lines formed by Sella-Nasion and by Nasion-Point A (Steiner 1953).

Sella-Nasion-Point B (SNB): the posterior-inferior angle formed by the intersection of the lines formed by Sella-Nasion and Nasion-Point B (Steiner 1953).

U1 to L1 (Interincisal Angle): the posterior angle formed at the intersection of the long axis of the upper incisor (UIA-UIE) and the log axis of the lower incisor (LIA-LIE) (Baumrind and Frantz 1971).

U1 to Nasion-A Point: the posterior angle determined by Nasion, the intersection of the line Nasion-Point $\mathrm{A}$ and the long axis of the (UIA-UIE) upper incisor (Baumrind and Frantz 1971:510).

U1 to Sella-Nasion: the posterior-inferior angle formed by the line through the long axis of $\mathrm{U} 1$ and the line defined by Sella and Nasion.

Y-Axis: the inferior-posterior angle formed by the intersection between the lines Porion-Orbitale and Sella-Gnathion, the point Gnathion (Baumrind and Frantz 1971).

\section{$\underline{\text { Cephalometric Linear Variables }}$}

A to Nasion Perpendicular: the linear distance from Point A to Nasion when projected perpendicular to the Frankfort Horizontal plane. This is a measure of horizontal maxillary position.

AO-BO: the distance along the occlusal plane of Point A and Point B projected perpendiculary on to the occlusal plane. Jacobson (1975) defined AOBO as the distance along the functional occlusal plane, but Downs' occlusal plane was used in this study.

B to Nasion Perpendicular: the linear distance from Point B to Nasion when projected perpendicular to the Frankfort Horizontal plane. This is a measure of horizontal mandibular position.

Condylion to Gnathion (Mandibular Length): the linear distance from

Condylion to Gnathion. This is a measure of mandibular length.

E-Plane: the line defined by soft tissue Pogonion and Pronasale.

L1 to Nasion- B Point : the distance in millimeters, from the point lower incisor edge to the line Nasion-B Point, measured perpendicular to that line (Baumrind and Frantz 1971:510).

Overbite: (positive overbite; vertical): the vertical overlap between the maxillary and mandibular incisors. This distance is measured perpendicular to Downs' occlusal plane.

Overjet: (horizontal): the distance between the labial surface of the mandibular incisors and the incisal edges of the maxillary incisors. The millimetric distance is measured parallel with Downs' occlusal plane. 
U1 to Nasion-A Point: a millimetric measurement of the relationship of the upper incisors to the line Nasion-A point (Steiner 1953).

\section{$\underline{\text { Data Acquisition }}$}

The cephalometric variables were obtained by the following method. Each cephalometric radiograph was scanned using a UMAX Powerlook III flatbed scanner at a resolution of $300 \mathrm{dpi}$ and 256 gray scale, and the scans were saved as TIFF files. The radiographic TIFF files were imported into Dolphin Imaging ${ }^{\circledR} 10.0$ (Dolphin Imaging and Management Solutions, Chatsworth, California) and traced using the program's digital cephalometric tracing package. A custom analysis was created with the "custom analysis builder" function in Dolphin Imaging to measure the 28 skeletodental angular and linear variables in this study. The measurements were exported from Dolphin Imaging into Microsoft Excel® 2007 (Microsoft, Inc. Redmond, Washington) and then into JMP® 7.0 (SAS Institute Inc., Cary, NC) for statistical analysis.

\section{$\underline{\text { Statistical Design }}$}

Data output from the Dolphin system were entered into an Excel spreadsheet (Microsoft Corporation, Redmond, WA) then transferred to the JMP statistical package (SAS Institute Inc., Cary, NC). Exploratory data analysis (Tukey 1977) was performed, searching for outliers; those due to technical errors were corrected.

Descriptive statistics were computed including arithmetic mean $(\bar{x})$, standard deviation (sd), standard error of mean (sem), lower and upper $95 \%$ confidence limit $\left(\mathrm{L}_{1}, \mathrm{~L}_{2}\right)$, sample size $(\mathrm{n})$, sample variance $\left(\mathrm{s}^{2}\right)$, skewness $\left(\mathrm{g}^{1}\right)$, kurtosis $\left(\mathrm{g}^{2}\right)$, coefficient of variation ( $\left.\mathrm{cv}\right)$, number of cases missing, maximum value, median value (50th percentile), and minimum value. Regarding the sample variance and skewness, the significance of these was not tested. All tests were two-tail evaluated at an alpha of 0.05 . No correction was made for multiple comparisons.

One sample t-tests (Sokal and Rohlf 1995) were used to evaluate whether a cephalometric variable changed systematically from the end of treatment and the recall examination. The null hypothesis was that the change from pre- to posttreatment examination was not statistically different from zero. In practice the changes were calculated in Excel, and the JMP statistical package was used to test whether the change differed from zero. That is, the actual mean change was tested against a hypothesized mean change of zero $(\mathrm{df}=\mathrm{n}-1)$. 
A repeated-measures mixed-model ANOVA design also was used since identically the same patients were followed pre- to posttreatment. This repeatedmeasures aspect of the analysis was combined with the two factorial groupings, namely (A) sex of the subjects was included in the model since boys' and girls' craniofacial growth vectors are apparently different (Harris 2001; van der Linden 1986) and (B) a treatment effect (i.e., Edgewise treatment with or without the MARA) was in the model since the fundamental topic of this research was whether the MARA provides an identifiable difference over conventional fixed treatment alone.

\section{$\underline{\text { ANOVA Design }}$}

Factorial analysis of variance (ANOVA) was the primary statistical approach used to test for group differences. Three sets of variables were evaluated, (1) pretreatment status, (2) posttreatment status, and (3) the intreatment changes. A two-way ANOVA was used, where one axis was sex of the patient (since males are bigger and tend to grow more than females), and the other axis was the variable itself. This is a completely randomized design, so both are evaluated against the same error mean square (Winer et al. 1991).

We initially used the patient's 'age at the start of treatment' as a covariate in the statistical tests, but the tight grouping of ages produced no effect, so the model was simplified.

The ANOVA results disclose whether the variable in question differs in average size between the two treatment groups while controlling for sexual dimorphism. These results are supplemented, as needed, by one-sample t-tests evaluating whether a particular mean differs significantly from zero (e.g., Sokal and Rohlf 1995). These (two-tail) t-tests evaluate whether the in-treatment change in a variable differs from the null hypothesis of "no difference," in which case the change is effectively centered on zero.

\section{The Reliability of Head Film Measurements}

Cephalograms are used extensively in orthodontics in the assessment of growth and malocclusions and the determination of treatment outcomes. A cephalogram is an enlarged two-dimensional image of an object that is threedimensional. 


\section{Landmark Identification}

Several standard anatomic landmarks are located on cephalograms and used to make angular and linear measurements. There unavoidably is some error in the location of all of these landmarks (Houston 1983). Downs (1956) reported that there is known uncertainty in accurately locating Porion (1956). Downs singled out Porion for comment because its location in the petrous portion of the temporal bone made it particularly difficult to locate, especially before the routine use of rare-earth intensifying films.

In a study by Aelbers and Dermaut (1996) the measurement error was quantified for several commonly used angular and linear measurements. The standard error of $1^{\circ}$ was found for the angular measurements and the standard error for linear measurements did not exceed $1 \mathrm{~mm}$, except when measuring mandibular length (Condylion-Gnathion) where the error is $2.3 \mathrm{~mm}$ (1996). One of the problems in measuring mandibular length lies in the uncertainty of accurately locating the head of the condyle because of the lack of radiographic contrast of the structures in the lateral view.

Headfilm measurements involve error (Baumrind and Frantz 1971). Two general classes of error occur in the estimation of cranial proportion from head films. The first class of error is called errors of projection; this results from the head film being a two dimensional shadow of a three dimensional object. The rays that produce the shadow are divergent originating from a very small source, so headfilms are always distorted enlargements. The second class of error is termed errors of identification. Baumrind and Frantz (1971) conducted a study to check operator reliability in the identification of standard cephalometric landmarks. The sample included 20 lateral skull headfilms, selected at random from 122 cases, from the University of California, School of Dentistry between 1954 and 1964. Each film was traced by five postgraduate orthodontic residents, and 16 standard cephalometric landmarks were identified. The study identified five sources of error: representativeness of headfilms, representativeness of judges, machine errors in point location, errors in superimposition of tracings, and errors in location of the reference planes (Baumrind and Frantz 1971). The study found that errors in landmark identification are too great to be overlooked, the magnitude of error varies from landmark to landmark, and the distribution of errors for most landmarks is not random; rather, each landmark has its own characteristics and envelope of error (Baumrind and Frantz 1971). Some landmarks have "confounding noise" from adjacent structures; examples are the lower incisor apex, apices of the roots of posterior teeth, and the positions of various points on the condyle (Baumrind and Frantz 1971). In their study, Porion was defined as the mechanical point (a radiopaque dot on the headholder) rather than the anatomic location. Sella has a unique identification 
problem because it involves the visual estimation of the center of the hypophysis. The identification of Nasion produced a large number of gross errors, which is detrimental since several angular relationships in a cephalometric analysis are based on the Sella-Nasion line. Baumrind and Frantz suggest routinely replicating each landmark to reduce error in landmark identification (1971).

\section{Conventional Angular and Linear Measures}

Baumrind and Frantz (1971) conducted a follow-up study that determined the effects of errors in landmark location on the values of the linear and angular measures of some commonly used head film analyses. There are three types of errors in angular and linear head film measurement: errors of projection, errors of landmark identification, and mechanical errors in drawing lines between points on tracings and in measuring with a ruler or protractor (Baumrind and Frantz 1971). To reduce the errors of projection, angular rather than linear measurements should be made whenever possible. Angular measurements remain the same in spite of the enlargement factor. There are three considerations that determine the impact that the error in identification of a specific landmark has on the linear and angular values involving that particular landmark. First is the actual magnitude of error involved in accurately identifying the specific landmark. Second is the linear distance on the tracing between the landmark point and the point(s) representing other landmarks to which it is connected in the process of computing a given dimension. Third is the direction in which the line between two landmarks intersects the envelope of error for each landmark.

The most striking observation in their study is the realization that angular and linear measurements from cephalograms contain considerable error. These errors are far too great to simply overlook or ignore. There are several steps that can be taken to reduce errors in measurement of the cephalogram. First, the clinician must realize that error exists, so care and concentration are required to obtain a reliable tracing. Second, the clinician needs to embrace the routine use of replicated measurements. With the advent of computer technology, replicated measurements can be done easily.

Houston (1983) looked at the sources of error in angular and linear cephalometric measurements. Cephalometric measurements made with their defining points lying in a plane parallel to the film are not distorted, but if the points are not parallel to the film the linear and angular measurements are affected. It is imperative the operator position the patient so the ear rod axis is perpendicular to the midsagittal plane of the head. Systematic errors can occur when two series of radiographs are analyzed by different observers because the 
observers have different concepts of identifying particular landmarks (Houston 1983). Houston agrees with Baumrind and Frantz (1971) that the greatest source of error involves difficulties in identifying certain landmarks and the ambiguity of some of the definitions. Observer's identification of particular landmarks even vary at different times.

As data are collected, the design of the experiment should include looking for outliers (Tukey 1977). If measurements are more than three standard deviations away from the mean it might be the expression of normal variation, but usually these are the result of "incorrect identification of a point or misreading an instrument" (Houston 1983:385). Random errors can be reduced if measurements are repeated and averaged. This should be done by repeating the tracings and not merely re-measuring the tracings since the greatest error is in point identification (Houston 1983). 


\section{CHAPTER IV. RESULTS}

\section{Comparisons at Start of Treatment}

Comparability of the two samples at the start of treatment is essential. As noted earlier, we matched each of the MARA-treated cases with an Edgewiseonly case based on six criteria, specifically (1) the patient's sex, (2) SNA, (3) SNB, (4) ANB, (5) NAP, and (6) FMA. The strategy was to match the two groups on a case-by-case basis using these cephalometric criteria specifically directed at anteroposterior relationship of the maxilla and mandible. Descriptive statistics, by treatment and sex, for cephalometric variables evaluated at the start of treatment can be found in Appendix B.

We judged the time-intensive effort at matching to be quite successful (Table 2) in that the probabilities (P values) of all five cephalometric variables to be strongly nonsignificant.

It is worth interjecting an explanation of how the statistics in Table 2 were derived. Each row (variable) in the table involves a separate analysis. Repeatedmeasures ANOVA was used to test for a difference between the two treatment groups (MARA-Edgewise and Edgewise-only) and the result is listed in the table under "treatment". Since there was a mix of boys and girls in the sample we also constructed a separate contrast to test whether the cephalometric variables differed by sex- probably due to the groups' growth differences (e.g., Ursi). Thirdly, the "interaction" columns evaluated whether the two sexes responded differently to the treatments. That is, additivity of treatment and sex effects is assumed by ANOVA, and the interaction term tests whether this is indeed true. Of note, none of the interaction terms approached statistical significance.

Conventional fixed orthodontic appliances do not just move teeth. Instead, it has been appreciated and warranted a name change of the American Journal of Orthodontics to include "and Dentofacial Orthopedics" - that orthopedic corrections can also be achieved. "Orthopedics" in this sense involves modifications of the basal bone supporting the teeth proper. Several mechanical devices, such as interarch elastics, headgear and now TADs (temporary anchorage devices), are capable of altering bone growth as well as moving teeth.

It is easy to visualize how a restraining (compressive) force can reduce growth. It is not so obvious from the literature whether tension (or hyperpropulsion) can appreciably enhance bone growth. Meikle's review (2007) 
Table 2. Results of mixed-model analysis of variance tests to evaluate the comparability of the matched samples at the start of treatment.

\begin{tabular}{|c|c|c|c|c|c|c|}
\hline \multirow[b]{2}{*}{ Variable } & \multicolumn{2}{|c|}{$\underline{\text { Sex }}$} & \multicolumn{2}{|c|}{ Treatment } & \multicolumn{2}{|c|}{ Interaction } \\
\hline & $\mathrm{F}$ & $\mathrm{P}$ & $\mathrm{F}$ & $\mathrm{P}$ & $\mathrm{F}$ & $\mathrm{P}$ \\
\hline Z Angle & 0.37 & 0.5456 & 5.98 & 0.0164 & 0.28 & 0.5991 \\
\hline E Plane & 0.37 & 0.5467 & 3.86 & 0.0525 & 0.21 & 0.6475 \\
\hline Y-Axis & 0.41 & 0.5254 & 0.06 & 0.7996 & 0.13 & 0.7208 \\
\hline NAP & 0.09 & 0.7708 & 0.14 & 0.7096 & 0.02 & 0.8753 \\
\hline SNA & 0.37 & 0.5425 & 0.37 & 0.5419 & 0.13 & 0.7177 \\
\hline SNB & 0.00 & 0.9954 & 0.17 & 0.6787 & 0.07 & 0.7894 \\
\hline ANB & 1.58 & 0.2114 & 0.26 & 0.6127 & 0.04 & 0.8488 \\
\hline $\mathrm{AOBO}$ & 4.36 & 0.0393 & 0.32 & 0.5756 & 0.35 & 0.5543 \\
\hline FMA & 0.05 & 0.8307 & 2.25 & 0.1365 & 0.62 & 0.4316 \\
\hline IMPA & 2.19 & 0.1421 & 0.05 & 0.8208 & 2.68 & 0.1047 \\
\hline FMIA & 1.54 & 0.2176 & 0.60 & 0.4421 & 1.17 & 0.2824 \\
\hline $\mathrm{OP} / \mathrm{FH}$ & 0.07 & 0.7965 & 1.49 & 0.2253 & 0.06 & 0.8100 \\
\hline U1/L1 & 1.22 & 0.2716 & 0.64 & 0.4248 & 0.14 & 0.7115 \\
\hline U1/SN & 1.46 & 0.2298 & 2.86 & 0.0941 & 0.99 & 0.3224 \\
\hline U1/NA & 0.30 & 0.5830 & 1.34 & 0.2493 & 0.11 & 0.7405 \\
\hline $\mathrm{U} 1 / \mathrm{NA}(\mathrm{mm})$ & 0.59 & 0.4448 & 3.46 & 0.0658 & 0.31 & 0.5775 \\
\hline L1/NB & 0.81 & 0.3695 & 0.04 & 0.8397 & 1.24 & 0.2681 \\
\hline L1/NB (mm) & 2.11 & 0.1498 & 0.76 & 0.3849 & 0.46 & 0.4983 \\
\hline L1/AP & 1.44 & 0.2336 & 0.05 & 0.8177 & 2.21 & 0.1407 \\
\hline L1/ AP (mm) & 0.15 & 0.6999 & 0.50 & 0.4795 & 0.49 & 0.4835 \\
\hline A-Na-Perp & 0.00 & 0.9773 & 2.34 & 0.129 & 0.06 & 0.8091 \\
\hline B-Na-Perp & 1.03 & 0.3138 & 5.60 & 0.0199 & 0.01 & 0.9435 \\
\hline Fac Ang & 0.23 & 0.6327 & 4.77 & 0.0314 & 0.01 & 0.9176 \\
\hline Md Lgth & 5.48 & 0.0213 & 0.81 & 0.3693 & 0.11 & 0.7385 \\
\hline S-A (mm) & 7.27 & 0.0082 & 1.64 & 0.2038 & 0.65 & 0.4238 \\
\hline S-B (mm) & 4.64 & 0.0338 & 0.10 & 0.7483 & 0.76 & 0.3844 \\
\hline Overbite & 0.35 & 0.5576 & 2.99 & 0.0867 & 0.26 & 0.6119 \\
\hline Overjet & 1.04 & 0.3107 & 5.87 & 0.0173 & 3.55 & 0.0624 \\
\hline
\end{tabular}


notes that the evidence for growth augmentation is fairly compelling in animal trials, but still controversial in humans (Cochrane review).

The purpose of the present study was to test whether the use of one sort of mandible hyperpropulsion device, the MARA, measurably augments mandibular growth, thereby creating a more orthognathic bony facial profile than fixed appliance treatment alone. As detailed previously, this retrospective clinical study measured the skeletal and dental changes during treatment in a cohort treated with a MARA and Edgewise appliance compared to a matched sample of patients treated with Edgewise appliances alone. The remainder of this chapter describes the treatment changes in these two groups.

\section{$\underline{\text { Extractions }}$}

The extractions of some teeth can be used in orthodontic treatment to correct problems, with one obvious problem being tooth-size arch-size discrepancies (TSAD). In this study, extractions as part of orthodontic therapy were far more common in the Edgewise group than in the MARA group. Only premolars were extracted and the frequency was 69\% (35/51 cases) in the Edgewise group compared to $4 \%$ (2/51 cases) in the MARA group.

\section{$\underline{\text { Time in Treatment }}$}

A slightly longer treatment time occurred in the MARA-Edgewise group. The patients treated with the MARA began at an average age of 12.6 years and completed treatment at an average age of 15.4 years, with a total duration of 2.8 years (Table 1). The patients treated with Edgewise-only began at an average age of 13.1 years and completed treatment at an average age of 15.6 years, with a total duration of 2.5 years (Table 1 ).

This difference of about 3 months in average treatment time is consistent enough across samples that it approaches statistical significance $(P=0.07)$, so even though the MARA commonly is used in conjunction with fixed appliances (rather than as a separate phase of treatment), it does take about 5 months longer, at least in this comparison.

\section{Comparisons at End of Treatment}

In the full cephalometric analysis (Table 2), a few of the variables (4/28) do differ significantly between the two groups. The largest differences 
statistically are (1) a more prominent lip (the $\mathrm{Z}$ angle) in the Edgewise group and (2) greater overjet in the Edgewise group. These differences seem unimportant because (1) we did not match on these characteristics and (2) lip position and overjet are controlled by tooth position, which we view as minor, readily managed issues that do not involve the underlying basal bone relationships. Descriptive statistics, by treatment and sex, for cephalometric variables evaluated at the end of treatment can be found in Appendix C.

The "proof of the pudding" is the set of comparisons at the end of treatment (Table 3). Adjunctive use of the MARA was supposed to improve interjaw anteroposterior relationship vis-à-vis Edgewise treatment alone. If true, the improvements ought to be discernible cephalometrically. There are four key variables here, namely (1) NAP, (2) ANB, (3) AOBO and (4) the facial angle (Na$\mathrm{A}-\mathrm{Pg})$, because these define various aspects of the maxilla-mandibular relationships in the AP plane.

These two groups display comparable mean NAP angles $(\mathrm{P}=0.24)$. Riolo et al. (1974) found a mean NAP angle of about $4^{\circ}$, which is quite similar to that found here for these treated cases, especially given Riolo's large standard deviation $\left(\operatorname{SD~} 5^{\circ}-6^{\circ}\right)$.

The angle ANB (Steiner 1953) is marginally smaller in the MARAEdgewise sample $(\mathrm{P}=0.02)$, though the roughly one-degree difference between group means is not compelling. Still, the final mean ANB angle of $3^{\circ}$ in the MARA-Edgewise sample is more desirable than the mean of $4^{\circ}$ in the Edgewiseonly sample.

The Wits discrepancy (AOBO) (Jacobson 1975) is another informative measure of inter-jaw harmony. At face value, mean AOBO is smaller (more harmonious) in the MARA-Edgewise sample, but the intergroup differences is not significant statistically $(\mathrm{P}=0.22)$.

Down's angle of facial convexity (Na-Pg to FH) (Downs 1956) is another measure of interarch relationships. The larger the angle, the more prominent the chin is when viewed in profile. The mean facial angle is significantly larger in the MARA-Edgewise group $\left(\bar{x}=87^{\circ}\right)$ than in the Edgewise-only group $\left(\bar{x}=85^{\circ}\right)$. Riolo et al. (1974) list the average angle at 16 years of age as $81-85^{\circ}$ (larger in girls), so the means found in the present study for both groups are satisfactory outcomes, though again, the MARA does seem to produce more orthognathic facial profiles. 
Table 3. Results of mixed-model analysis of variance tests to evaluate the comparability of the matched samples at the end of treatment.

\begin{tabular}{|c|c|c|c|c|c|c|}
\hline \multirow[b]{2}{*}{ Variable } & \multicolumn{2}{|c|}{$\underline{\text { Sex }}$} & \multicolumn{2}{|c|}{ Treatment } & \multicolumn{2}{|c|}{ Interaction } \\
\hline & $\mathrm{F}$ & $\mathrm{P}$ & $\mathrm{F}$ & $\mathrm{P}$ & $\mathrm{F}$ & $\mathrm{P}$ \\
\hline Z Angle & 4.61 & 0.0344 & 0.00 & 0.9930 & 0.12 & 0.7344 \\
\hline E Plane & 4.28 & 0.0414 & 0.69 & 0.4090 & 0.03 & 0.8664 \\
\hline Y-Axis & 1.33 & 0.2513 & 0.11 & 0.7356 & 0.03 & 0.8524 \\
\hline NAP & 3.16 & 0.0787 & 1.13 & 0.2901 & 0.23 & 0.6300 \\
\hline SNA & 0.04 & 0.8487 & 0.26 & 0.6089 & 0.04 & 0.8484 \\
\hline SNB & 0.80 & 0.3735 & 0.39 & 0.5322 & 0.65 & 0.4220 \\
\hline ANB & 1.60 & 0.2082 & 5.18 & 0.0250 & 1.37 & 0.2450 \\
\hline $\mathrm{AOBO}$ & 0.12 & 0.7260 & 2.18 & 0.1430 & 3.61 & 0.0603 \\
\hline FMA & 0.03 & 0.8740 & 1.12 & 0.2935 & 0.08 & 0.7748 \\
\hline IMPA & 0.33 & 0.5647 & 0.19 & 0.6671 & 0.76 & 0.3859 \\
\hline FMIA & 0.44 & 0.5110 & 0.35 & 0.5563 & 0.36 & 0.5483 \\
\hline $\mathrm{OP} / \mathrm{FH}$ & 0.00 & 0.9474 & 9.47 & 0.0027 & 0.01 & 0.9078 \\
\hline U1/L1 & 2.13 & 0.1479 & 13.01 & 0.0005 & 0.11 & 0.7432 \\
\hline U1/SN & 4.93 & 0.0286 & 12.19 & 0.0007 & 1.00 & 0.3197 \\
\hline U1/NA & 4.34 & 0.0399 & 13.71 & 0.0004 & 0.76 & 0.3864 \\
\hline $\mathrm{U} 1 / \mathrm{NA}(\mathrm{mm})$ & 4.51 & 0.0361 & 24.52 & $<0.0001$ & 0.94 & 0.3350 \\
\hline L1/NB & 0.00 & 0.9471 & 2.39 & 0.1253 & 0.08 & 0.7791 \\
\hline L1/NB (mm) & 0.70 & 0.4040 & 3.44 & 0.0665 & 0.09 & 0.7700 \\
\hline L1/AP & 1.69 & 0.1972 & 4.25 & 0.0420 & 0.07 & 0.7966 \\
\hline L1/ AP (mm) & 0.58 & 0.4498 & 20.15 & $<0.0001$ & 1.06 & 0.3059 \\
\hline A-Na Perp & 3.40 & 0.0683 & 3.75 & 0.0557 & 0.03 & 0.8549 \\
\hline B-Na Perp & 3.51 & 0.0641 & 10.96 & 0.0013 & 0.54 & 0.4645 \\
\hline Fac Ang & 0.70 & 0.4034 & 9.72 & 0.0024 & 0.12 & 0.7344 \\
\hline Md Lgth & 18.56 & $<0.0001$ & 0.05 & 0.8195 & 0.03 & 0.8615 \\
\hline S-A (mm) & 23.34 & $<0.0001$ & 1.88 & 0.1734 & 0.02 & 0.8770 \\
\hline S-B (mm) & 23.60 & $<0.0001$ & 4.57 & 0.0351 & 1.02 & 0.3159 \\
\hline Overbite & 0.66 & 0.4173 & 19.97 & $<0.0001$ & 0.56 & 0.4579 \\
\hline Overjet & 1.43 & 0.2348 & 6.49 & 0.0124 & 0.10 & 0.7513 \\
\hline
\end{tabular}




\section{Nature of the Correction}

A criticism of the conventional treatment of Class II malocclusion is that the "wrong" jaw is treated. Since "growing" a mandible to correct the Class II problem has not been an option, treatment has focused on the restraint of maxillary growth to achieve inter jaw harmony, but this can increase convexity of the facial profile. Descriptive statistics, by treatment and sex, for the intreatment changes in cephalometric variables can be found in Appendix D.

To specifically assess forward (ventral) position of the two jaws, the position of Points A and B were measured relative to FH and Nasionperpendicular (Table 3).

The Edgewise-only sample had A Point a half-millimeter behind Nasionperpendicular at the start of treatment, and A Point was remodeled backwards about $2 \mathrm{~mm}$ during treatment, finishing at a mean of $-2.5 \mathrm{~mm}$. This distal remodeling of A Point probably was achieved with uprighting of the incisor but also with the use of Class II elastics, and, in some cases, HPHG (high pull headgear).

In the MARA-Edgewise sample, A Point was, on the average, about a halfmillimeter ahead of Nasion-perpendicular at the start of treatment, it was remodeled backwards at an average of $1.7 \mathrm{~mm}$ during treatment, finishing at one $\mathrm{mm}$ behind Nasion. In other terms, A Point was located at the same mesiodistal position in both groups at the start of treatment $(\mathrm{P}=0.13)$, and it was moved backwards roughly $2 \mathrm{~mm}$ in both groups using Edgewise mechanics (with a nonsignificant difference between groups; $\mathrm{P}=0.60$ ). Final position was "better" (closer to Nasion-perpendicular) in the MARA-Edgewise group, but not significantly so statistically $(\mathrm{P}=0.08)$.

It can be noted, then, that both treatments restrain forward growth of the maxilla, but the extent is slightly but not significantly less with adjunctive use of the MARA $(P=0.08)$.

As for the mandible, B Point was more retrusive in the Edgewise-only group at the start of treatment $(\bar{x}=10.4 \mathrm{~mm})$, and it fell further behind during treatment, with a mean distance of $-11.2 \mathrm{~mm}$ at the end. The net loss of B Point relative to Nasion-perpendicular was about $1 \mathrm{~mm}$. Use of the MARA caused the advancement of B Point from a mean of $-8.1 \mathrm{~mm}$ to $-7.4 \mathrm{~mm}$ and a mean change of $-0.8 \mathrm{~mm}$ during the course of treatment. It is arguable, then, that (A) the MARA prevented worsening of the mandibular retrognathia seen in the other group and (B) the MARA actually caused a net forward movement of B Point. 
A mean forward movement of one millimeter seems rather trivial and probably not detectable visually- but treatment with the MARA ought also to be credited with preserving the millimeter of loss noted with Edgewise treatment alone, so the real benefit is a forward movement of about $2 \mathrm{~mm}$ with the MARA. This forward movement of B Point with the MARA accounts for much of the correction (reduction) in ANB, which decreased significantly more when using the MARA adjunct.

\section{In-Treatment Changes}

Status of the cephalometric variables at the end of treatment is the most telling aspect of the analysis, but, for completeness the in-treatment changes are also reviewed (Table 4). Mean in-treatment changes, by treatment and sex, of the cephalometric variables can be found in Appendix E.

The change (reduction) in SNA was statistically the same in the two groups. This is not surprising since both cases were treated with Edgewise mechanics and the action of the MARA should not affect remodeling at A Point. This lack of a group difference is confirmed by the measurement of A Point to Nasion-perpendicular, where both groups experienced the same amount of reduction.

Effects of the MARA (compared to Edgewise mechanics alone) seem to be localized to the mandible. There was significantly greater forward growth with the MARA as measured by (1) greater change in ANB $(P=0.02),(2)$ reduction in $\mathrm{AOBO}(\mathrm{P}=0.02)$, and (3) forward growth of $\mathrm{B}$ Point relative to Nasionperpendicular $(\mathrm{P}=0.04)$. In addition, the distance Sella-B Point increased significantly more with the MARA $(P=0.004)$. Whether the greater correction of overjet seen with the MARA $(\mathrm{P}=0.0005)$ may also be relevant (though part of this dental correction may involve group differences in finishing the occlusion).

In passing, it can be noted that most of these group differences are rather small, but certainly detectable statistically. All of the tests reported in this study were evaluated as two-tail tests. Arguably, the P values could be halved since the direction of the improvements with the MARA, if discernible, are known. On the other hand, most of the significant differences (e.g., ANB, AOBO, overjet) are interrelated measures of the same skeletal response. No correction was made in this study for multiple comparisons. 
Table 4. Results of mixed-model analysis of variance tests to evaluate the comparability of the matched samples for the in-treatment changes.

\begin{tabular}{|c|c|c|c|c|c|c|}
\hline \multirow[b]{2}{*}{ Variable } & \multicolumn{2}{|c|}{$\underline{\text { Sex }}$} & \multicolumn{2}{|c|}{ Treatment } & \multicolumn{2}{|c|}{ Interaction } \\
\hline & $\mathrm{F}$ & $\mathrm{P}$ & $\mathrm{F}$ & $\mathrm{P}$ & $\mathrm{F}$ & $\mathrm{P}$ \\
\hline Z Angle & 1.49 & 0.2254 & 5.81 & 0.0181 & 0.04 & 0.8455 \\
\hline E Plane & 0.61 & 0.4359 & 6.12 & 0.0154 & 0.03 & 0.8601 \\
\hline Y-Axis & 1.37 & 0.2439 & 0.03 & 0.8717 & 0.89 & 0.3475 \\
\hline NAP & 8.98 & 0.0035 & 1.01 & 0.3176 & 0.86 & 0.3563 \\
\hline SNA & 0.66 & 0.4190 & 0.02 & 0.8839 & 0.10 & 0.7500 \\
\hline SNB & 3.38 & 0.0689 & 4.32 & 0.0403 & 1.36 & 0.2468 \\
\hline ANB & 11.73 & 0.0009 & 5.78 & 0.0181 & 3.42 & 0.0676 \\
\hline AOBO & 3.36 & 0.0700 & 0.69 & 0.4074 & 5.94 & 0.0166 \\
\hline FMA & 0.26 & 0.6112 & 0.11 & 0.7406 & 0.39 & 0.5326 \\
\hline IMPA & 0.75 & 0.3900 & 0.04 & 0.8369 & 0.52 & 0.4713 \\
\hline FMIA & 0.48 & 0.4888 & 0.07 & 0.7913 & 0.34 & 0.5636 \\
\hline $\mathrm{OP} / \mathrm{FH}$ & 0.05 & 0.8233 & 8.08 & 0.0054 & 0.21 & 0.6468 \\
\hline U1/L1 & 0.00 & 0.9693 & 8.61 & 0.0042 & 0.27 & 0.6025 \\
\hline U1/SN & 0.00 & 0.9912 & 12.24 & 0.0007 & 0.20 & 0.6565 \\
\hline U1/NA & 0.91 & 0.3412 & 11.57 & 0.0010 & 0.10 & 0.7508 \\
\hline $\mathrm{U} 1 / \mathrm{NA}(\mathrm{mm})$ & 0.74 & 0.3932 & 26.44 & $<0.0001$ & 0.04 & 0.8332 \\
\hline $\mathrm{L} 1 / \mathrm{NB}$ & 0.94 & 0.3350 & 1.27 & 0.2634 & 0.78 & 0.3787 \\
\hline L1/NB (mm) & 0.75 & 0.3890 & 5.22 & 0.0245 & 0.84 & 0.3611 \\
\hline L1/AP & 0.02 & 0.9020 & 1.51 & 0.2214 & 1.19 & 0.2790 \\
\hline L1/ AP (mm) & 0.03 & 0.8617 & 18.88 & $<0.0001$ & 2.54 & 0.1143 \\
\hline A-Na Perp & 8.26 & 0.0050 & 0.66 & 0.4196 & 0.00 & 0.9471 \\
\hline B-Na Perp & 2.25 & 0.1365 & 4.01 & 0.0480 & 0.92 & 0.3407 \\
\hline Fac Ang & 0.37 & 0.5471 & 2.95 & 0.0891 & 0.40 & 0.5264 \\
\hline Md Lgth & 7.95 & 0.0058 & 0.46 & 0.5000 & 0.14 & 0.7052 \\
\hline S-A (mm) & 17.29 & $<0.0001$ & 0.17 & 0.6768 & 0.86 & 0.3550 \\
\hline S-B (mm) & 21.81 & $<0.0001$ & 8.93 & 0.0035 & 0.11 & 0.7413 \\
\hline Overbite & 1.00 & 0.3209 & 0.10 & 0.7476 & 0.78 & 0.3797 \\
\hline Overjet & 0.32 & 0.5709 & 12.82 & 0.0005 & 3.37 & 0.0696 \\
\hline
\end{tabular}




\section{CHAPTER V. DISCUSSION}

Class II malocclusions are common, with approximately one-third of youths in the United States having this parasagittal malrelationship (Brunelle et al. 1996; Cozza et al. 2006). In a typical orthodontic practice, the correction of Class II malocclusions may constitute almost half of the cases (Franchi and Baccetti 2001). A Class II malocclusion can stem from diverse skeletodental disharmonies of the two jaws (Moyers et al. 1980), but a common occurrence is insufficient mandibular length rather than excess size of the maxilla (Graber et al. 2005; Moyers et al. 1980). There are several ways for the specialist to treat this sort of skeletal disharmony.

The treatment modalities available to correct a Class II malocclusion include an array of extra-oral appliances, arch expansion appliances, various dental extraction patterns, functional jaw orthopedic appliances, and, most intrusively, orthognathic surgery to advance the mandible (McNamara and Brudon 1993). Each treatment modality "differs in its effect on the skeletal structures of the craniofacial region, sometimes accelerating or limiting the growth of the various structures involved" (McNamara and Brudon 1993). The MARA, mandibular anterior repositioning appliance, is a fixed functional appliance that was introduced in 1991 by Douglass Toll of Germany and was further developed by Jim Eckhart, of California, ORMCO and AOA Laboratory.

To date, there is only one study evaluating the effects of the MARA on patients with Class II malocclusion, and this was written by Pangrazio-Kulbersh et al. in 2003. The purpose of the present study was to add information through the analysis of cephalometric radiographs about the skeletodental effects of the MARA when used in conjunction with conventional Edgewise treatment. The goal of this cephalometric study was to identify any skeletal benefits obtained from the added use of the MARA in the treatment of skeletal Class II subjects. The present author matched each MARA-treated case with an Edgewise-only case based on six criteria, specifically (1) the patient's sex, (2) SNA, (3) SNB, (4) ANB, (5) NAP, and (6) FMA. This matched-pair design permits the statistical detection of smaller differences than with group comparisons.

Dr. Wasson, the single specialist who treated all of the MARA cases, uses a three-tier treatment approach for his treatment of Class II malocclusions. Patients who fall in the first tier usually are in the early mixed dentition stage, with high mandibular plane angles (FMA), openbite tendencies, and deleterious oral habits (e.g., tongue thrusts, digit habits). These patients usually have excessive overjet with constricted or underdeveloped dental arches due to habits and/or poor lip competence. A patient's treatment typically consists of Fränkel 
II (FR-2) wear for approximately 22 hours a day for 1 year followed by a retention phase consisting of wearing the appliance for 8 to 12 hours a day. Once all of the permanent teeth have fully erupted, Edgewise appliances finish the treatment.

The second tier of treatment is aimed at patients exhibiting Class II division 2 malocclusions. These patients have low mandibular plane angles (FMA), deepbite tendencies and a forward growth pattern. These patients are older, 9 to 11 years of age, as compared to the Tier 1 patients but are still in the mixed dentition. First, the Class II division 2 malocclusion is converted to a Class II division 1 malocclusion to facilitate treatment. The patient will then wear a Bionator full time for 9 to 12 months with 6-to 8-week appointment intervals to assess their progress. Once correction is achieved, the appliance is worn only at night for retention while the rest of the permanent dentition erupts. Next, Edgewise appliances are used to detail and finish the case.

The third treatment strategy is aimed at Class II patients in the early permanent dentition (11 to 14 years of age). This group of patients often is treated with fixed functional appliances such as the Herbst appliance or MARA (i.e., mandibular advancement repositioning appliance). These patients can have brackets placed on the upper four incisors to aid in aligning the incisors. Alternatively, full brackets and bands can be placed concomitantly to expedite treatment. Since these patients are older there is less growth that can be harnessed by the appliance, so more of the Class II correction is achieved by dental rather than skeletal change (Harris 2001; Harris and McKinney 2001).

\section{$\underline{\text { Premolar Extractions }}$}

The extraction of specific teeth can be used in orthodontic treatment to correct problems, with one obvious problem being tooth-size arch-size discrepancies (TSASD). There is a perception in the general public that extractions are "wrong" and parents may want their children to be treated without extractions, but there seems to be no scientific basis for retaining all of the premolars. In this study, extractions, as part of orthodontic therapy, were far more common in the Edgewise group than in the MARA group. Premolars were the only teeth extracted in these subjects, and the frequency was $69 \%(35 / 51$ cases) in the Edgewise group compared to a mere $4 \%$ (2/51 cases) in the MARA group.

What does this rather dramatic difference in proportion mean? At one extreme, teeth are functionally unimportant with today's highly-processed diet, where only trivial mastication is required (English et al. 2002). Indeed, only in very recent generations has the expectation of edentulism with dentures 
diminished in the population (Kelly et al. 1967; Kelly and Van Kirk 1973). Arguably, teeth are functionally unimportant, and the loss of teeth in the midarch, which is out of sight even on high-smile, has no esthetic consequence, especially with the extraction space being closed. Certainly there is no comparable concern by the public for retention of third molars (Eklund and Pittman 2001).

Alternatively, there clearly is a popular perception that prophylactic extraction of teeth for orthodontic treatment somehow mutilates the adolescent's dentition. There is no evidence that masticatory efficiency is diminished in those treated with premolar extractions. Iatrogenic consequences of premolar extractions are much lower than for wisdom teeth (Anonymous 1980), and risks for third molars are themselves quite low. Another argument, that premolar extractions increase the risk of TMD have been show to be specious (Behrents and White 1992).

Arguments against premolar extractions seem to be wholly perceptual rather than evidence-based. However, insofar as orthodontic treatment is generally elective, perception of the patient and more critically the adolescent's parents seem to have a real effect on which treatment plan-and which specialist - is chosen.

\section{$\underline{\text { Time in Treatment }}$}

A slightly longer mean treatment time occurred in the MARA-Edgewise group, but the difference is not statistically significant $(P=0.07)$. The patients treated with the MARA began treatment at an average age of 12.6 years and completed treatment at an average age of 15.4 years, with a duration of 2.8 years (Table 1). Patients treated with Edgewise-only began at an average age of 13.1 years and completed treatment at an average age of 15.6 years, with a duration of 2.5 years (Table 1 ). Foremost, the difference is insignificant statistically, so there is no real difference to prefer one approach over the other. It has been popular to discuss treatment "efficiency," which is essentially a code word for how fast (in months of treatment) a case can be completed. Shorter time is supposed to translate into fewer appointments and, thus, greater productivity and/or the opportunity to increase starts-per-year.

A benefit of the MARA over several other hyperpropulsive devices (Brudon and McNamara1993) is that the MARA can be fitted and used in conjunction with other fixed appliances, so there is no sense of two phases of treatment. The MARA achieves whatever hyperpropulsion that it can while the Edgewise appliances are achieving their goals. 


\section{Pattens of Skeletodental Change}

It has been argued that functional appliance therapy corrects skeletal disharmonies primarily by improving mandibular position in relation to the maxillary arch. In contrast, conventional appliances, with use of Class II elastics and/or headgear wear, achieves much of the skeletal correction by restraining maxillary growth. Therefore, the MARA can be a better treatment option because it does not treat the "wrong" jaw by restraining the maxilla. Instead, the intent of the MARA is to encourage forward growth of the retrognathic mandible to fit the maxilla. Cephalometric tracings (superimpositions) can be found in Appendix F.

The unfortunate paradox, as noted by Meikle $(2006,2007)$ and others is that substantive mandibular growth can be achieved in laboratory animals (Bryan and McNamara 1987; Elgoyhen et al. 1972; Graber and McNamara 1975; Luder 1987), while clinical trials have been far less convincing. Indeed, the outcomes of all three randomized clinical trials on this topic (O'Brien et al. 2003; Tulloch et al. 1998; Wheeler et al. 2006) fail to show any enhancement of mandibular growth in children. Random assignment of children to treatments versus differential diagnosis - is a weakness of the RCT "gold standard," but the lack of any augmented mandibular growth is an important consideration.

One consideration that does not seem to have been thought through is that all laboratory animals are nominally "Class I" since skeletal malocclusions are rare. These normative growers may not possess the factors that cause mandibular retrognathia. Lylse Johnson is fond of the phrase that "them that has gets," meaning that clinically it is easier to obtain growth in those who do not exhibit a growth deficiency.

Numerous skeletodental variables were tested in the Results chapter for completeness, but the key purpose of the MARA is to enhance mandibular growth, and it is useful to focus here specifically on this claim. Of the variables measured in this study, five are most relevant to evaluating the skeletal facial profile at the end of treatment, namely ANB, NAP, AOBO, A to Nasionperpendicular, and B to Nasion-perpendicular. The acid test in this study involves whether these skeletal variables are appreciably more harmonious in the MARA sample at the end of treatment.

These findings are depicted in Figure 11. Tests for sexual dimorphism are not significant for these five dimensions, so sexes are pooled to simplify discussion. ANB is significantly smaller at the end of treatment when the MARA was employed. ANB has long been touted as a key measure of facial harmony. 


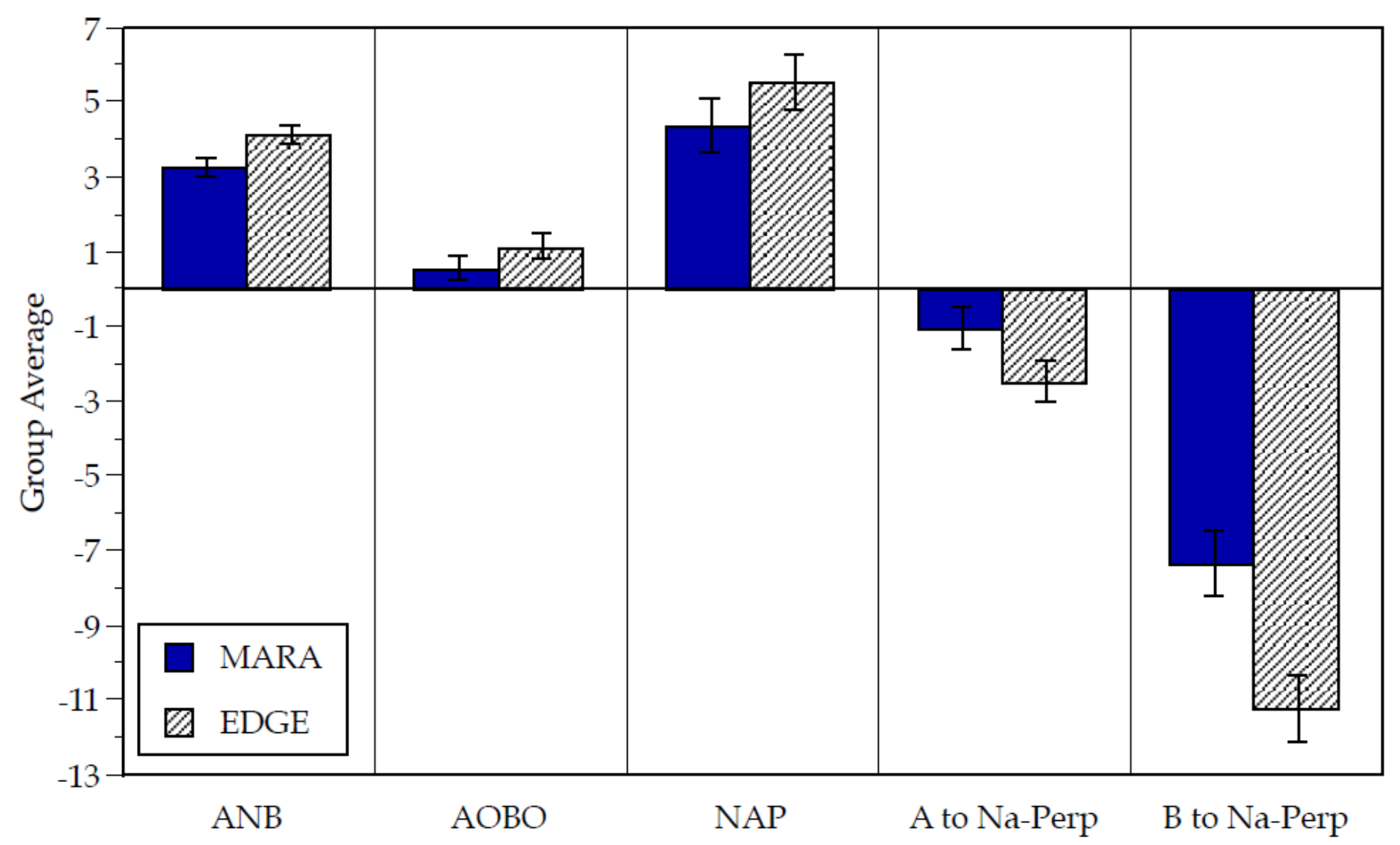

Figure 11. Means of 5 variables, by treatment, at the end of treatment. Error bars are \pm sem. By one-way ANOVA, just 2 of these dimensions are different statistically (ANB and B to Na-Perp). 
(Riedel 1952; Steiner 1953) because it relates the upper, middle, and lower thirds of the face to one another. Since ANB was the same in the two groups at the start of treatment (Table 2), this change argues in favor of the anticipated orthopedic effect of the MARA. Operationally, the elbow device attached to the upper molar does indeed propulse the mandible forward enough on jaw-closure that the mandible grows forward more during treatment than in the Edgewise-only group. The site of action is not known specifically from this study, but suggestions are that (1) the condylar process itself was elongated, (2) shape of the condylar capitulum was altered to permit forward relocation of the mandible, and/or (3) the glenoid fossa was remodeled (anterior resorption; posterior apposition) to position the condyle more ventrally on the temporal bone. Eachor some combination of these three alterations might be involved, but none can be verified cephalometrically. Instead, histological studies of laboratory animals are needed (Voudouris et al. $2003 \mathrm{a}, \mathrm{b}$ ). Analogous with other, better studied hyperpropulsion appliances suggests that all three of these processes could be involved to some extent.

Notably, NAP does not corroborate this conclusion. NAP is Down's angle of convexity (1948), and, while it is about one degree less in the MARA group, this is not significantly different statistically. The obvious difference between ANB and NAP is Pogonion is substituted for B Point in the Angle of Convexity, and the chin point (Pogonion) is more variable among people than B Point, thus accounting for the lack of significance here.

$\mathrm{AOBO}$ (the Wits appraisal) is another valuable measure of jaw harmony (Jacobson 1988), but using the occlusal plane rather than some facial line for inference can produce quite different results. Average AOBO did not differ at the start of treatment in these two groups $(\bar{x}=3.3 \mathrm{~mm})$, nor did it differ at the end of treatment $(0.8 \mathrm{~mm})$ because the MARA in itself has no effect on Points A and $\mathrm{B}$. $\mathrm{AOBO}$ was reduced to a comparable - and quite desirable mean - in both treatments, but in different ways. With the MARA, B Point moved forward (augmented growth) so A Point did not need to be restrained as much to achieve the same overjet at the end of treatment. Edgewise treatment alone involved greater maxillary restraint. AOBO ends up the same in the two groups, but by holding back the upper arch in the Edgewise sample compared to promoting mandibular growth (or, at least, remodeling) with the MARA.

These treatment differences also are evident when looking at a change in $A$ and $B$ relative to Nasion-perpendicular (Figures 12 and 13). A Point was brought back about a millimeter with the MARA but appreciably more $(\bar{x}=2.5$ $\mathrm{mm}$ ) with the Edgewise appliance alone. It is supposed that A Point itself remodels in response to alterations of upper incisor positions and angulations. A Point also moves dorsally due to the orthopedic forces of headgear and of Class 


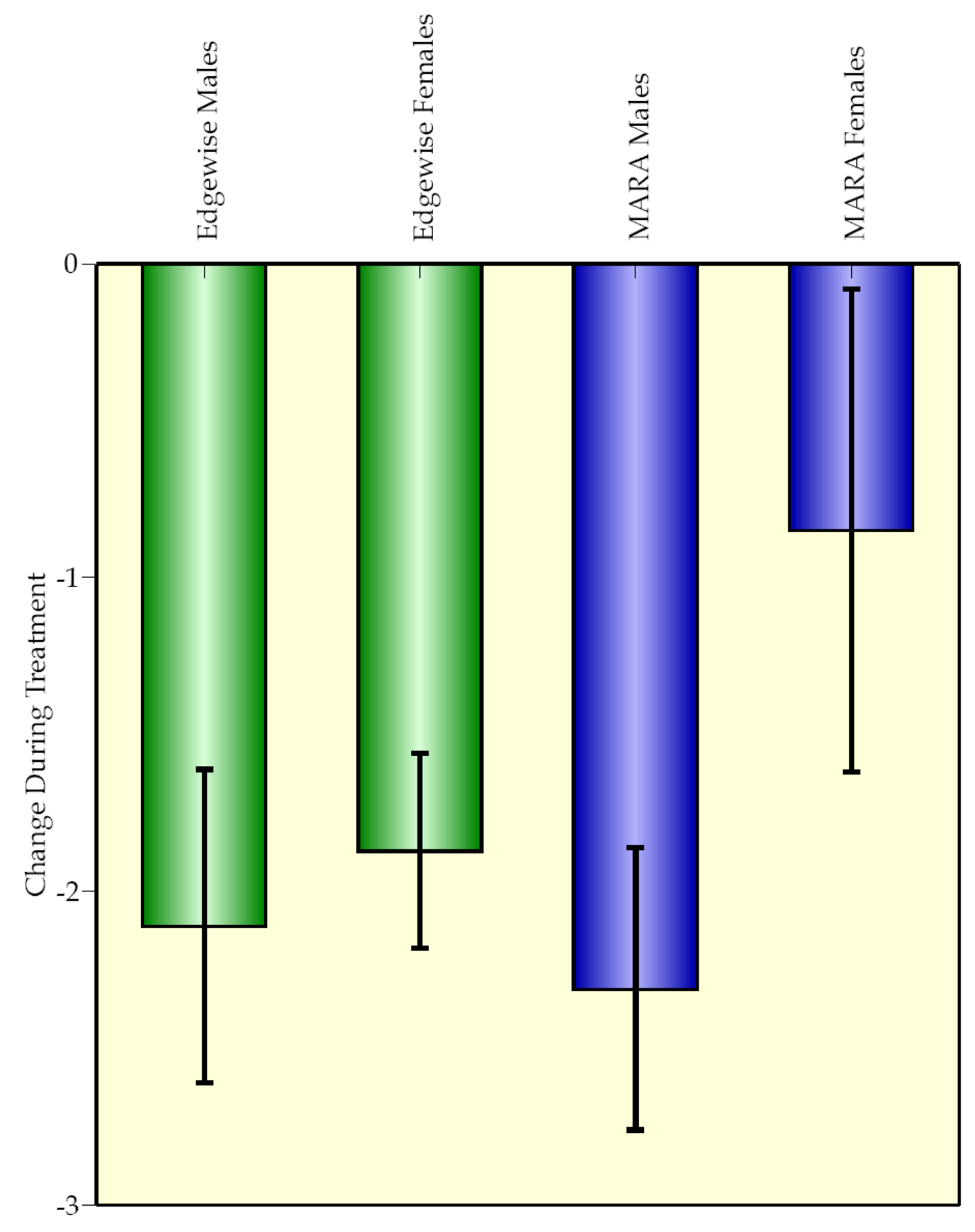

Figure 12. Mean in-treatment changes in A Point to Nasion-perpendicular, by treatment and by sex. Error bars are \pm one standard error of the mean. 


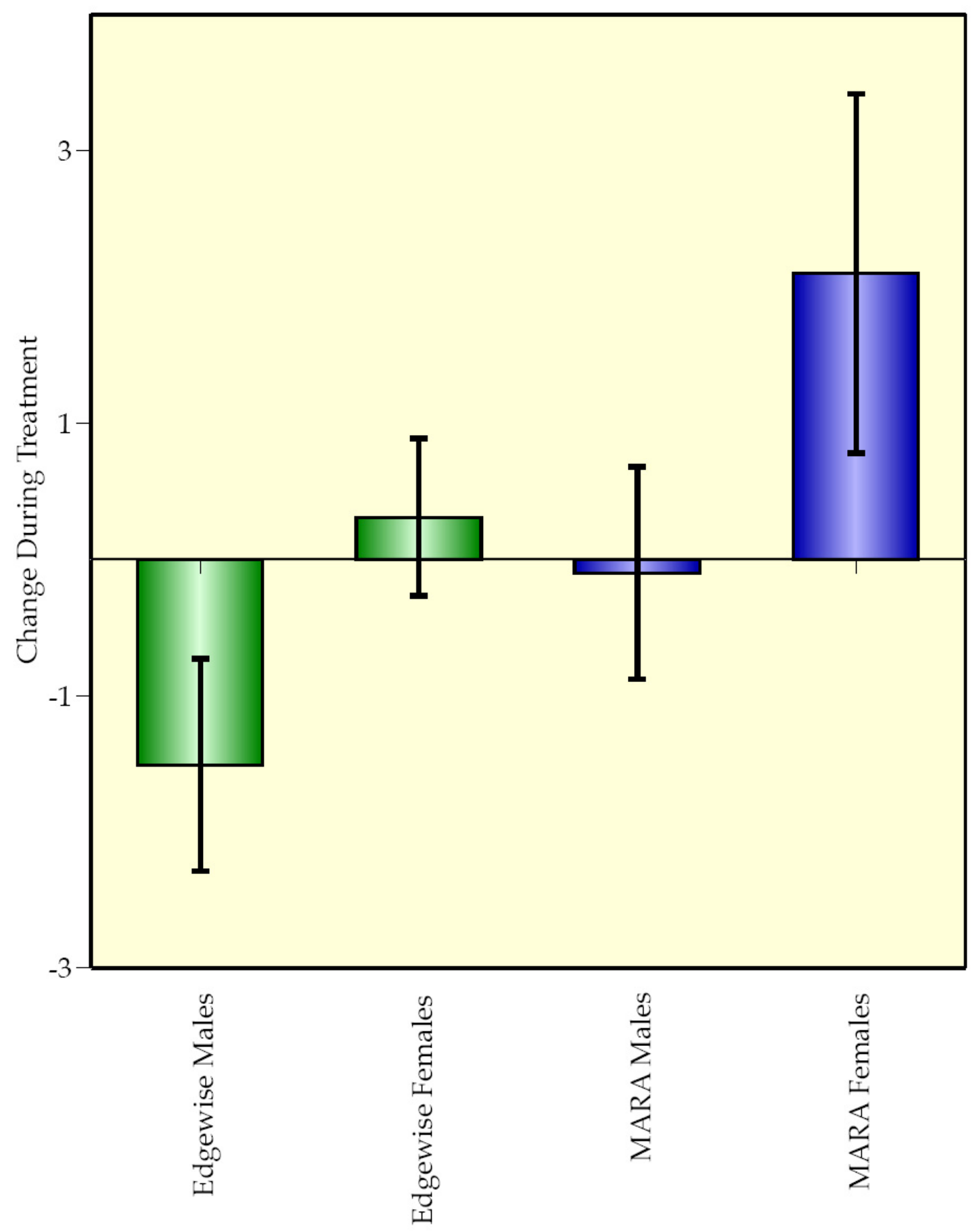

Figure 13. Mean in-treatment changes in B Point to Nasion-perpendicular, by treatment and by sex. Error bars are \pm one standard error of the mean. 
II elastics. Forces from these devices compress the circummaxillary sutures, with actual resorption of bone in the sutural margins (as opposed to simply restraining normal forward growth).

The difference between the means is about $1.5 \mathrm{~mm}$ : At the end of treatment, A Point was just $1.0 \mathrm{~mm}$ behind Nasion-perpendicular line in the MARA-Edgewise sample, but $2.5 \mathrm{~mm}$ behind in the Edgewise-alone group. This again makes the point that conventional treatment typically reduces the jaw discrepancy by bringing the maxilla back towards the lower jaw and, thereby, running the risk of increasing the subject's facial convexity.

The group difference is larger for B Point. At the end of treatment (Figure 13), B Point is, on the average, $11 \mathrm{~mm}$ behind the Nasion-perpendicular line in the Edgewise-only group. The mean is $7 \mathrm{~mm}$ in the MARA-Edgewise group, evidently because the MARA enhanced mandibular growth. "Enhanced growth" is a loaded term in some circles because (as we have noted) RCT studies do not substantiate this claim. This MARA sample used here was selected on the basis of differential diagnosis, not randomization. In this study, the average difference between treatments places the mandible (B Point) $4 \mathrm{~mm}$ closer to Nasion-perpendicular (a highly significant difference).

It will be of interest to determine where the skeletal difference occurs, probably some combination of condylar growth and remodeling of the fossa, but the key clinical issue is that the subjects were treated to a more orthognathic profile when using the MARA.

The MARA actually produced a net forward movement of B Point and prevented worsening of the mandibular retrognathia as seen in the Edgewise group. The MARA and Edgewise subjects were treated to a mean ANB of $3^{\circ}$ and $4^{\circ}$, respectively, which according to Steiner (1953) and Riolo et al. (1974) are within normal limits. From a cephalometric standpoint, both treatments were successful though the MARA did achieve a more orthognathic profile compared to the Edgewise-only group. Both treatment groups were improved cephalometrically at the end of treatment, but in different ways.

The aim of the MARA is directed towards enhancing the growth of the mandible and has no immediate effect on the maxilla. Both treatments restrain forward growth of the maxilla using Edgewise mechanics, but the extent is slightly but not significantly less with the adjunctive use of the MARA. This difference could be attributed to the clinician's technique, since the two treatment groups were treated by different clinicians. 


\section{$\underline{\text { Profile Attractiveness }}$}

In today's society, the public is consumed with beauty, and enhanced facial attractiveness is a driving force for people to seek orthodontic treatment (Shaw 1988). However, there are many aspects of facial esthetics that are outside the orthodontist's control, such as size and conformation of the person's nose, lip, chin, and body weight.

A study by Stock et al. (2006) concluded that growing Class II, division 1 patients typically can reach equivalent improvement in their profile when treated with either a Herbst appliance or headgear with fixed appliances only. Similarly, in a study by Fergus (2007), Edgewise treatment with and without a MARA produced the same esthetic profile when evaluated by experienced orthodontists, graduate orthodontic students, and lay people.

From an orthodontic standpoint, the MARA and the Edgewise subjects reach essentially the same endpoint. Skeletally, both groups finished with the same SNB angle of $76^{\circ}$. Both the MARA and Edgewise subjects finished with an acceptable ANB angle of $3^{\circ}$ and $4^{\circ}$, respectively, and with only $1^{\circ}$ difference between the groups the difference is not discernible clinically nor statistically. Thirdly, the angle of convexity (Na-A-Pg) decreased significantly to approximately the same point $\left(5^{\circ}\right)$ in both groups.

From a dental perspective, the effects of the MARA (in conjunction with Edgewise mechanics) are as follows:

- $\quad$ The interincisal angle (U1/L1) was slightly more acute compared to the Edgewise only cases (probably an operator preference).

- $\quad$ The incisor mandibular plane angle (IMPA) was more proclined at $98^{\circ}$ compared to the ideal angle of $88^{\circ}$ (Merrifield 1996).

- $\quad$ The Frankfort mandibular incisor angle (FMIA) was more acute than the ideal angle of $68^{\circ}$ (Merrifield 1996).

- $\quad$ The Lower 1 to A Point Pogonion angle (L1/AP) was larger than the ideal $22^{\circ}$.

- $\quad$ The Lower 1 to Nasion B Point angle (L1/NB) was larger than the ideal $25^{\circ}$.

- The Occlusal plane to Frankfort horizontal angle (OP/FH) was ideal at $9^{\circ}$.

These differences between treatments are dental, and they do not include key issues such as BSR, overjet, and overbite, where the two groups are indistinguishable. So too, none of these dimensions are attributable to effects of the MARA. The cephalometric analysis used here is based on the Department's battery of variables that is heavily weighted on dental issues that are directly 
under the orthodontist's control. We view these dental differences (1) as primarily due to operator preference and (2) not consequential to MARA treatment.

There are many benefits to the orthodontist and to the patient from use of the MARA. First, the patient has an immediate enhancement in their profile and an immediate reduction in overjet once the appliance is cemented in place. For many patients, the immediate profile enhancement can boost their selfconfidence. Secondly, the MARA patients retain more premolars than patients treated with fixed Edgewise appliances only. Lastly, the Class II correction is addressed early in treatment and does not incorporate patient compliance. 


\section{CHAPTER VI. SUMMARY AND CONCLUSIONS}

There has been considerable attention paid in the orthodontic literature to functional appliances, and the debate is still open as to whether they are a preferable treatment option for the growing Class II patient. The purpose of the present study was to add information through the analysis of cephalometric radiographs about the skeletodental effects of the MARA used in conjunction with conventional Edgewise treatment. The goal was to identify any skeletal benefits obtained from the use of the MARA in combination with Edgewise treatment of skeletal Class II subjects. The author matched each MARA-treated case with an Edgewise-only case based on six criteria, specifically (1) the patient's sex, (2) SNA, (3) SNB, (4) ANB, (5) NAP, and (6) FMA. By pairing each case, small differences could be discerned between the two treatments.

Both groups consisted of 51 subjects. The cephalometric analysis was performed with Dolphin Imaging ${ }^{\circledR}$ to measure 28 skeletodental angular and linear variables. Major findings are:

- There were significantly more premolar extractions in the Edgewise group $(69 \%)$ compared to the MARA subjects $(4 \%)$.

- $\quad$ Average treatment time was the same in both groups $(\bar{x}=2.8)$.

- A Point was remodeled backwards the same amount for both groups.

- There was more forward growth of B Point relative to Nasionperpendicular in the MARA-treated sample.

- $\quad$ The distance Sella-B Point increased significantly more with the MARA.

- There was a greater reduction of the angle ANB in the MARA group. 


\section{LIST OF REFERENCES}

Aelbers CMF, Dermaut LR. Orthopedics in orthodontics: part I, fiction or reality - a review of the literature. Am J Orthod Dentofacial Orthop 1996;110:513-9.

Allen-Noble PS. Clinical management of MARA. Allesee Orthodontic Appliances. July 2005.

[Anonymous] NIH consensus development conference for removal of third molars. J Oral Surg. 1980;38:235-6.

Arvystas MG. The rationale for early orthodontic treatment. Am J Orthod Dentofacial Orthop 1998;113:15-8.

Athanasiou AE. Orthodontic cephalometry. London: Mosby-Wolfe; 1995.

Baccetti T, Franchi L, McNamara JA Jr., Tollaro I. Early dentofacial features of class II malocclusion: a longitudinal study from the deciduous through the mixed dentition. Am J Orthod Dentofacial Orthop 1997;111:502-9.

Barton S, Cook P. Predicting functional appliance treatment outcome in Class II malocclusions - a review. Am J Orthod Dentofacial Orthop 1997;112:2826.

Baume LJ. Physiological tooth migration and its significance for the development of occlusion. I. The biogenetic course of the deciduous dentition. J Dent Res 1950;29:123-32.

Baume LJ. Physiological tooth migration and its significance for the development of occlusion. II. The biogenesis of accessional dentition. J Dent Res 1950;29:331-7.

Baumrind S, Frantz RC. The reliablilty of head film measurements, 1. Landmark identification. Am J Orthod 1971;60:111-27.

Baumrind S, Frantz RC. The reliability of head film measurements, 2. Conventional angular and linear measures. Am J Orthod 1971;60:505-17.

Behrents RG, White RA. TMJ research: responsibility and risk. Am J Orthod Dentofacial Orthop. 1992;101:1-3. 
Bishara SE, Hoppens BJ, Jakobsen JR, Kohout FJ. Changes in the molar relationship between the deciduous and permanent dentitions: a longitudinal study. Am J Orthod Dentofacial Orthop 2002;122:470-6.

Brunelle JA, Bhat M, Lipton JA. Prevalence and distribution of selected occlusal characteristics in the US population, 1988-1991. J Dent Res 1996;75:706-13.

Bussick TJ, McNamara JA Jr. Dentoalveolar changes associated with the pendulum appliance. Am J Orthod Dentofacial Orthop 2000;117:333-43.

Chen JY, Will LA, Niederman R. Analysis of efficacy of functional appliances on mandibular growth. Am J Orthod Dentofacial Orthop 2002;122:470-6.

Cohen L. Social psychological factors associated with malocclusion. Int Dent J 1970;20:643-53.

Cozza P, Baccetti T, Franchi L, Toffol LD, McNamara JA Jr. Mandibular changes produced by functional appliances in Class II malocclusion: a systematic review. Am J Orthod Dentofacial Orthop 2006;129:599.e1-12.

Dolce C, McGorray SP, Brazeau L, King GJ, Wheeler TT. Timing of Class II treatment: skeletal changes comparing 1-phase and 2-phase treatment. Am J Orthod Dentofacial Orthop 2007;132:481-9.

Downs WB. Variations in facial relationships: their significance in treatment and prognosis. Am J Orthod 1948;34:812-53.

Downs WB. Analysis of the dentofacial profile. Angle Orthod 1956;26:191-212.

Dudas M, Sassouni V. The hereditary componenets of mandibular growth, a longitudinal twin study. Angle Orthod 1973;43:314-23.

Eklund SA, Pittman JL. Third-molar removal patterns in an insured population. J Am Dent Assoc 2001;132:469-75.

Elgoyhen JC, Moyers RM, McNamara JA Jr., Riolo ML. Craniofacial adaptation of protrusive function in young rhesus monkeys. Am J Orthod 1972;62:46980.

Elsasser WA, Wylie WL. The craniofacial morphology of mandibular retrusion. J Anthrop 1943;6:461-73. 
English JD, Buschang PH, Throckmorton GS. Does malocclusion affect masticatory performance? Angle Orthod. 2002;72:21-7.

Feldmann I, Lundström F, Peck S. Occlusal changes from adolescence to adulthood in untreated patients with class II division 1 deepbite malocclusion. Angle Orthod 1999;69:33-8.

Franchi L, Baccetti T. Prediction of individual mandibular changes induced by functional jaw orthopedics followed by fixed appliances in Class II patients. Angle Orthod 2006;76:950-4.

Franchi L, Baccetti T. Class II malocclusion. In: McNamara JA Jr., Brudon WL, editors. Orthodontics and dentofacial orthopedics. Ann Arbor: Needham Press, 2001. p. 63-84.

Fränkel R, Fränkel C. Orofacial orthopedics with the function regulator. New York: Karger, 1989.

Gönner U, Özkan V, Jahn E, Toll DE. Effect of the MARA appliance on the position of the lower anteriors in children, adolescents, and adults with Class II malocclusion. J Orofac Orthop 2007;68:397-412.

Graber TM. Functional appliances. In: Graber TM, Vanarsdall RL, Vig KWL, editors. Orthodontics: current principles and techniques. St. Louis: CV Mosby; 2005. p. 493-542.

Graber TM. Treatment of patients in the mixed dentition. In: Graber TM, Vanarsdall RL, Vig KWL, editors. Orthodontics: current principles and techniques. St. Louis: CV Mosby; 2005. p. 543-77.

Hamilton DC. Early treatment - the emancipation of dentofacial orthopedics. In: Graber TM, Rakosi T, Petrovic AG, editors. Dentofacial orthopedics with functional appliances. St. Louis: CV Mosby; 1997. p. 319-35.

Harris DB, Enlow DH. A study of the postnatal growth of the mandible. Am J Orthod 1964;50:25-50.

Harris EF. Effects of patient age and sex on treatment: Correction of Class II cases with the Begg technique. Angle Orthod 2001;71:433-41.

Harrison JE, O'Brien KD, Worthington HV. Orthodontic treatment for prominent upper front teeth in children (review). Wiley, 2009. 
Houston WJ. The analysis of errors in orthodontic measurements. Am J Orthod 1983; 83:382-90.

Jacobson A. The "wits" appraisal of jaw disharmony. Am J Orthod 1975;67:12538.

Jacobson A. Update on the Wits appraisal. Angle Orthod. 1988;58:205-19.

Johnston LE. Growth and the Class II patient: rendering unto caesar. Sem Orthod 1998;4:59-62.

Kelly JE, Van Kirk LE, and Garst CC. Decayed, missing, and filled teeth in adults. Vital and Health Statistics, series 11, number 23. Rockville, MD: U.S. Department of Health, Education, and Welfare, 1967.

Kelly JE, Van Kirk LE. Total loss of teeth in adults, United States - 1960-1962. Vital and Health Statistics, series 11, number 27. Rockville, MD: U.S. Department of Health, Education, and Welfare, 1973.

King GJ, Wheeler TT, McGorray SP, Aiosa LS, Bloom RM, Taylor MG. Orthodontists perceptions of the impact of phase 1 treatment for Class II malocclusions on phase 2 needs. J Dent Res 1999;78:1745-53.

Luder HU. Growth direction in the mandibular condyle of prepubertal and pubertal monkeys (Macaca fascicularis) studied by morphometry and radioautography. Arch Oral Biol 1987;32:239-47.

McKinney JR, Harris EF. Influence of patient age and sex on orthodontic treatment: evaluations among Begg, Tweed, and Straightwire techniques. Am J Orthod Dentofacial Orthop 2001;120:530-41.

McNamara JA Jr. Components of Class II malocclusion in children 8-10 years of age. Angle Orthod 1981;51:177-202.

McNamara JA Jr. Neuromuscular and skeletal adaptations to altered function in the orofacial region. Am J Orthod 1973;64:578-606.

McNamara JA Jr., Brudon WL. Orthodontic and orthopedic treatment in the mixed dentition. Needham Press; 1993. 
McNamara JA Jr., Bryan FA. Long-term mandibular adaptations to protrusive function: an experimental study in Macaca mulatta. Am J Orthod Dentofacial Orthop 1987;92:98-108.

McNamara JA Jr., Graber LW. Mandibular growth in the rhesus monkey (Macaca mulatta). Am J Phys Anthropol 1975;42:15-24.

Maj G, Luzi C. Longitudinal study of mandibular growth between nine and thirteen years as a basis for an attempt of it prediction. Angle Orthod 1964;34:220-30.

Meikle MC. Remodeling the dentofacial skeleton: the biological basis of orthodontics and dentofacial orthopedics. J Dent Res 2007;86:12-24.

Merrifield LL. Differential diagnosis. Semin Orthod 1996;2:241-53.

Mitani H, Sato K. Comparison of mandibular growth with other variables during puberty. Angle Orthod 1992;62:217-22.

Moyers RE, Riolo ML, Guire KE, Wainright RL, Bookstein FL. Differential diagnosis of Class II malocclusions. Part 1 . Facial types associated with Class II malocclusions. Am J Orthod 1980;78;477-94.

O'Brien K, Wright J, Conboy F, Sanjie Y, Mandall N, Chadwick S, Connolly I, et al. Effectiveness of early orthodontic treatment with the Twin-block appliance: a multicenter, randomized, controlled trial. Part 1: Dental and skeletal effects. Am J Orthod Dentofacial Orthop. 2003;124:234-43.

Pangrazio-Kulbersh V, Berger JL, Chermak DS, Kaczynski R, Simon ES, Haerian A. Treatment effects of the mandibular anterior repositioning appliance on patients with Class II malocclusion. Am J Orthod Dentofacial Orthop 2003;123:286-95.

Proffit WR, Tulloch JF. Preadolescent class II problems: treat now or wait? Am J Orthod Dentofacial Orthop 2002;121:560-2.

Riedel RA. The relation of maxillary structures to cranium in malocclusion and in normal occlusion. Angle Orthod 1952;22:142-5. 
Riolo ML, Moyers RE, McNamara JA Jr., Hunter WS. An atlas of craniofacial growth: cephalometric standards from the University School Growth Study, the University of Michigan. Monograph 2, Craniofacial Growth Series. Ann Arbor: Center for Human Growth and Development, University of Michigan; 1974.

Shaw WC. Risk benefit appraisal in orthodontics. In: Moorrees CFA, van der Linden FPGM, editors. Orthodontics: evaluation and future. Nymegen, The Netherlands: University of Nymegen; 1988. p. 63-81.

Sokal RR, Rohlf FJ. Biometry: the principles and practice of statistics in biological research, 3rd ed. San Francisco: WH Freeman and Company; 1995.

Steiner CC. Cephalometrics for you and me. Am J Orthod 1953;39:729-55.

Stock S, Southard K, Sloss E, Qian F, Mann K. Profile attractiveness following treatment with the Herbst appliance versus headgear. J Dent Res 2006; special issue A:Abstract 790.

Tukey JW. Exploratory data analysis. Massachusetts: Addison-Wesley, Reading; 1977.

Tulloch JF, Proffit WR, Phillips C. Benefit of early Class II treatment: Progress report of a two-phase randomized clinical trial. Am J Orthod Dentofacial Orthop 1998;113:62-72.

Voudouris JC, Woodside DG, Altuna G, Angelopoulos G, Bourque PJ, Lacouture CY, Kuftinec MM. Condyle-fossa modifications and muscle interactions during Herbst treatment, Part 2. Results and conclusions. Am J Orthod Dentofacial Orthop. 2003b;124:13-29.

Voudouris JC, Woodside DG, Altuna G, Kuftinec MM, Angelopoulos G, Bourque PJ. Condyle-fossa modifications and muscle interactions during herbst treatment, part 1. New technological methods. Am J Orthod Dentofacial Orthop. 2003a;123:604-13.

Wahl N. Orthodontics in 3 millenia: Functional appliances to midcentury. Am J Orthod Dentofacial Orthop 2006;129:829-33.

Ward DM, Behrents RG, Goldberg JS. Temporomandibular synovial fluid response to altered mandibular positions. Am J Orthod Dentofacial Orthop 1990;98:22-8. 
Wheeler TT, McGorray SP, Dolce C, King GJ. The timing of Class II treatment. Am J Orthod Dentofacial Orthop 2006;129:S66-70.

Winer BJ, Brown DR, Michels KM. Statistical principles in experimental design, 3rd ed. New York: McGraw-Hill Book Company; 1991. 


\section{APPENDIX A. \\ SCHEMATIC DIAGRAMS OF LATERAL CEPHALOGRAMS SHOWING THE CONSTRUCTION OF VARIOUS ANGLES AND LINEAR MEASUREMENTS}




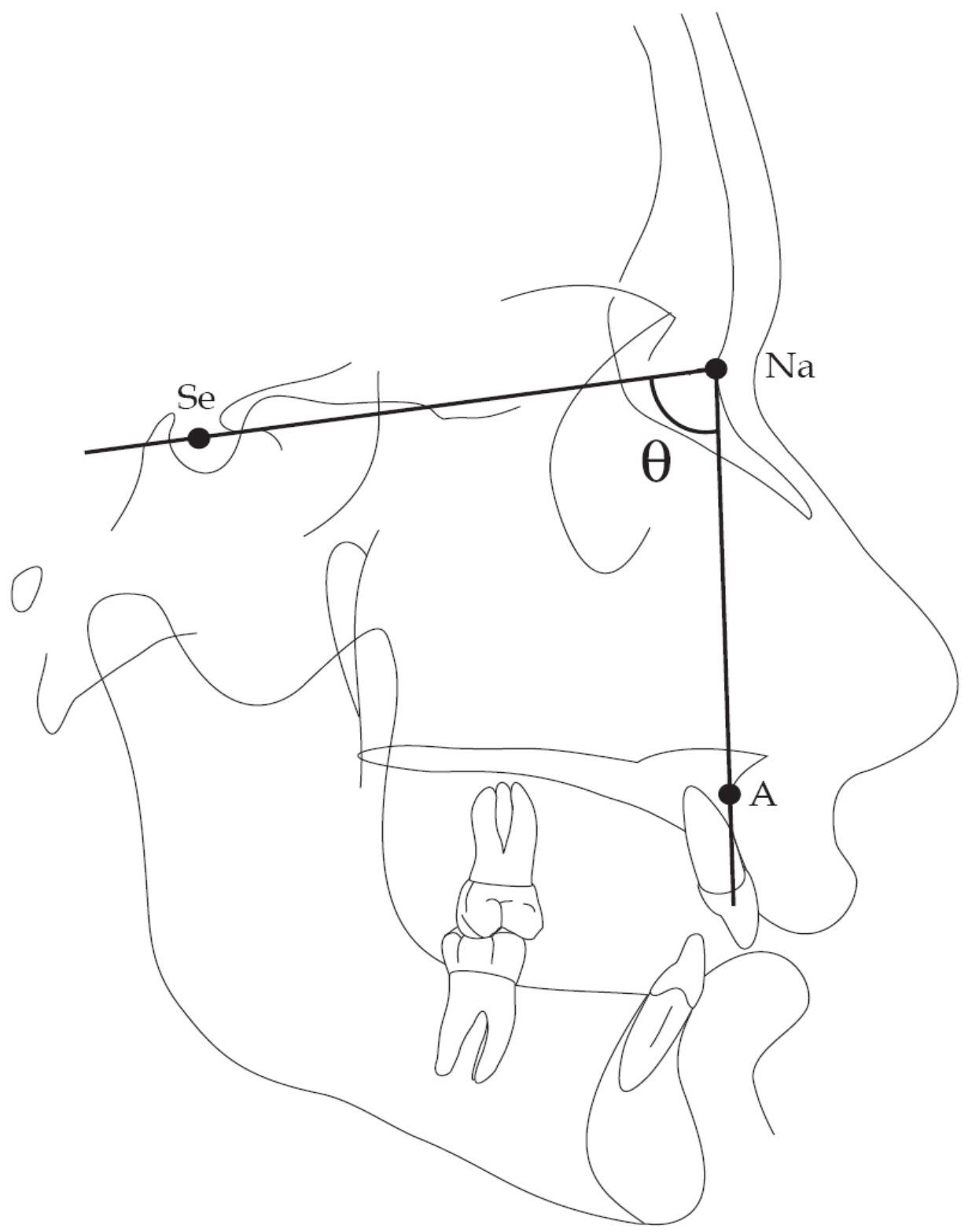

Figure A-1. Schematic tracing of a lateral cephalogram showing construction of the SNA angle. 


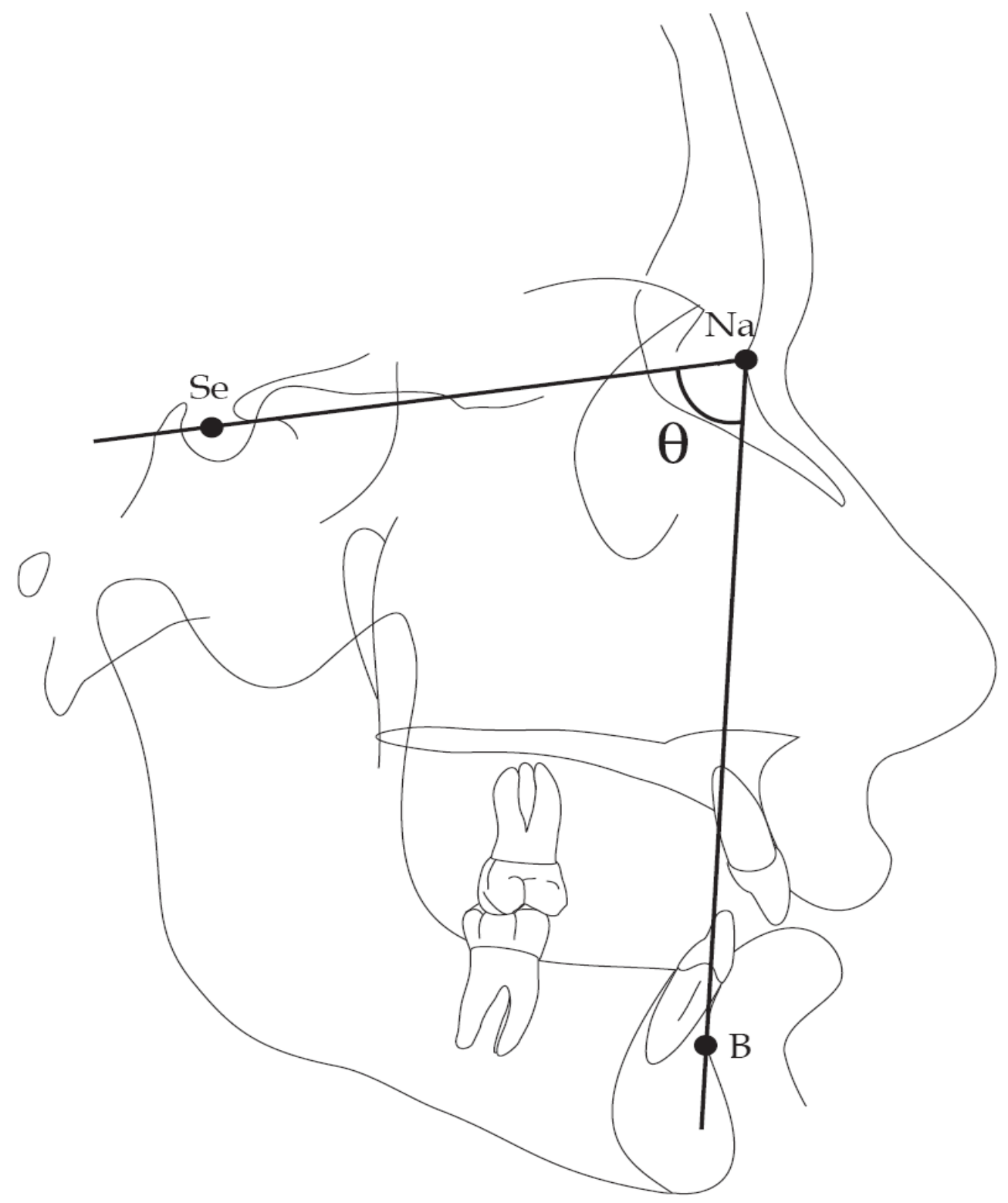

Figure A-2. Schematic tracing of a lateral cephalogram showing construction of the SNB angle. 


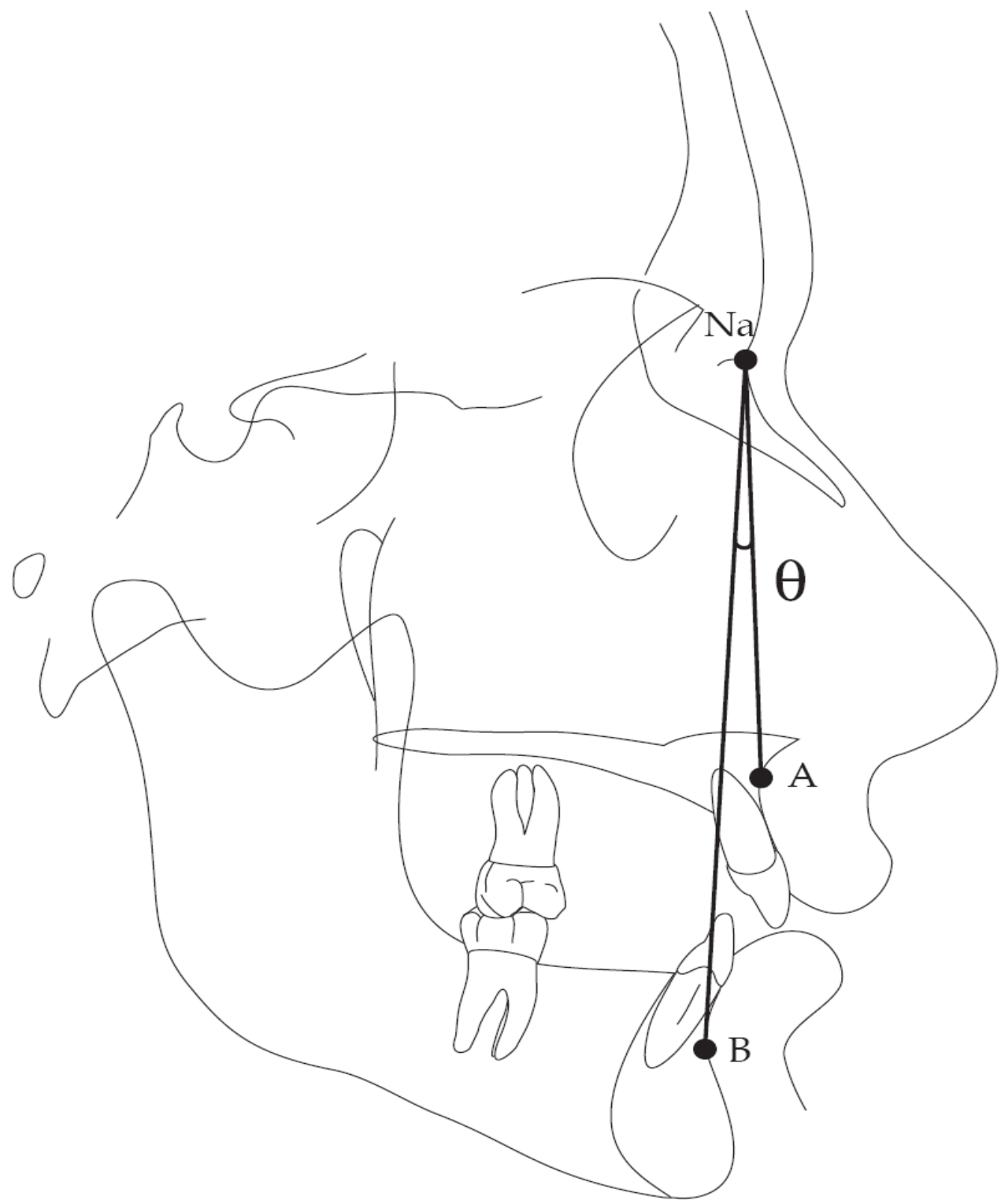

Figure A-3. Schematic tracing of a lateral cephalogram showing construction of the ANB angle. 


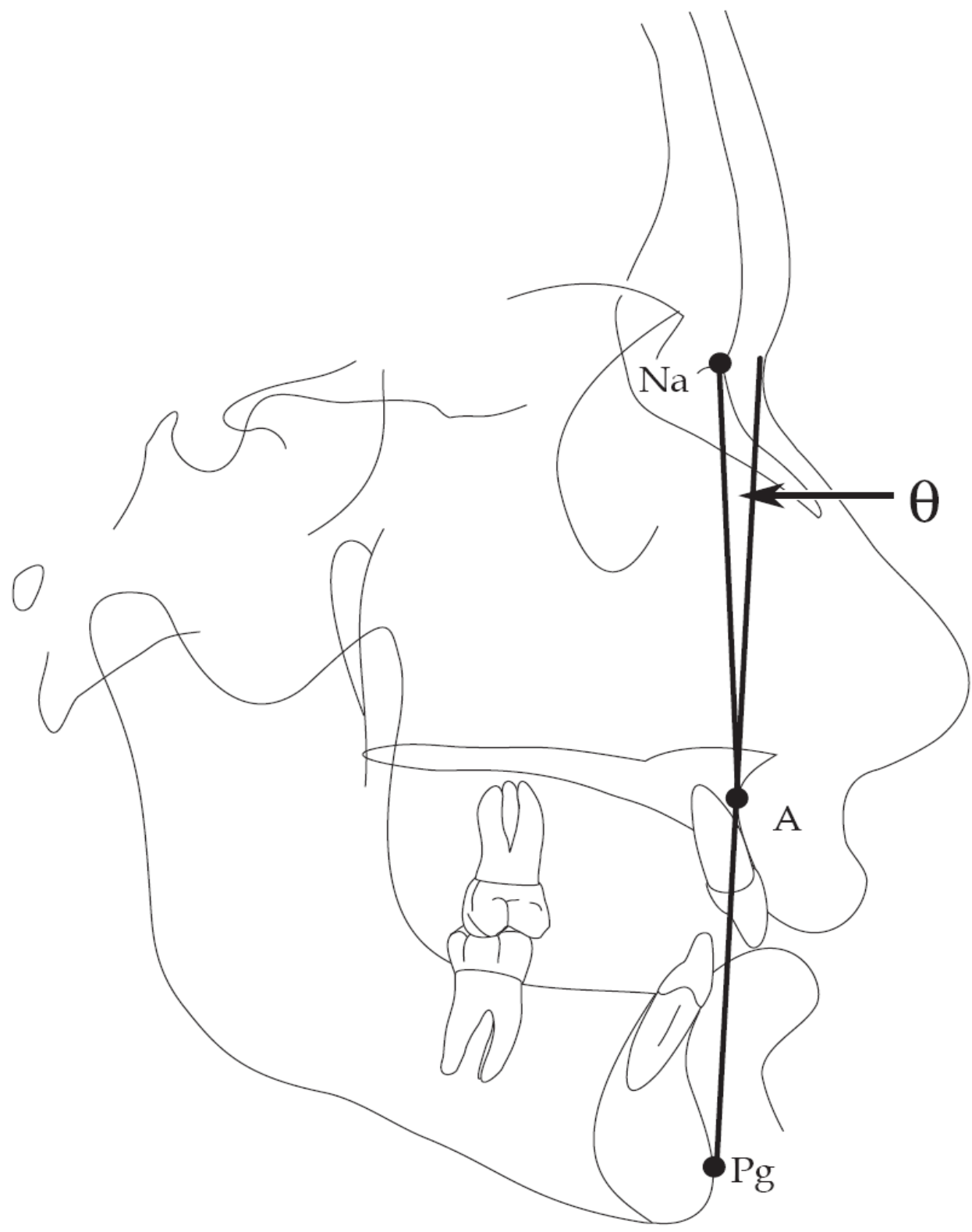

Figure A-4. Schematic tracing of a lateral cephalogram showing construction of the angle of convexity (NAP). This is the superior angle at the intersection of the Nasion-A and the A-Pogonion lines. 


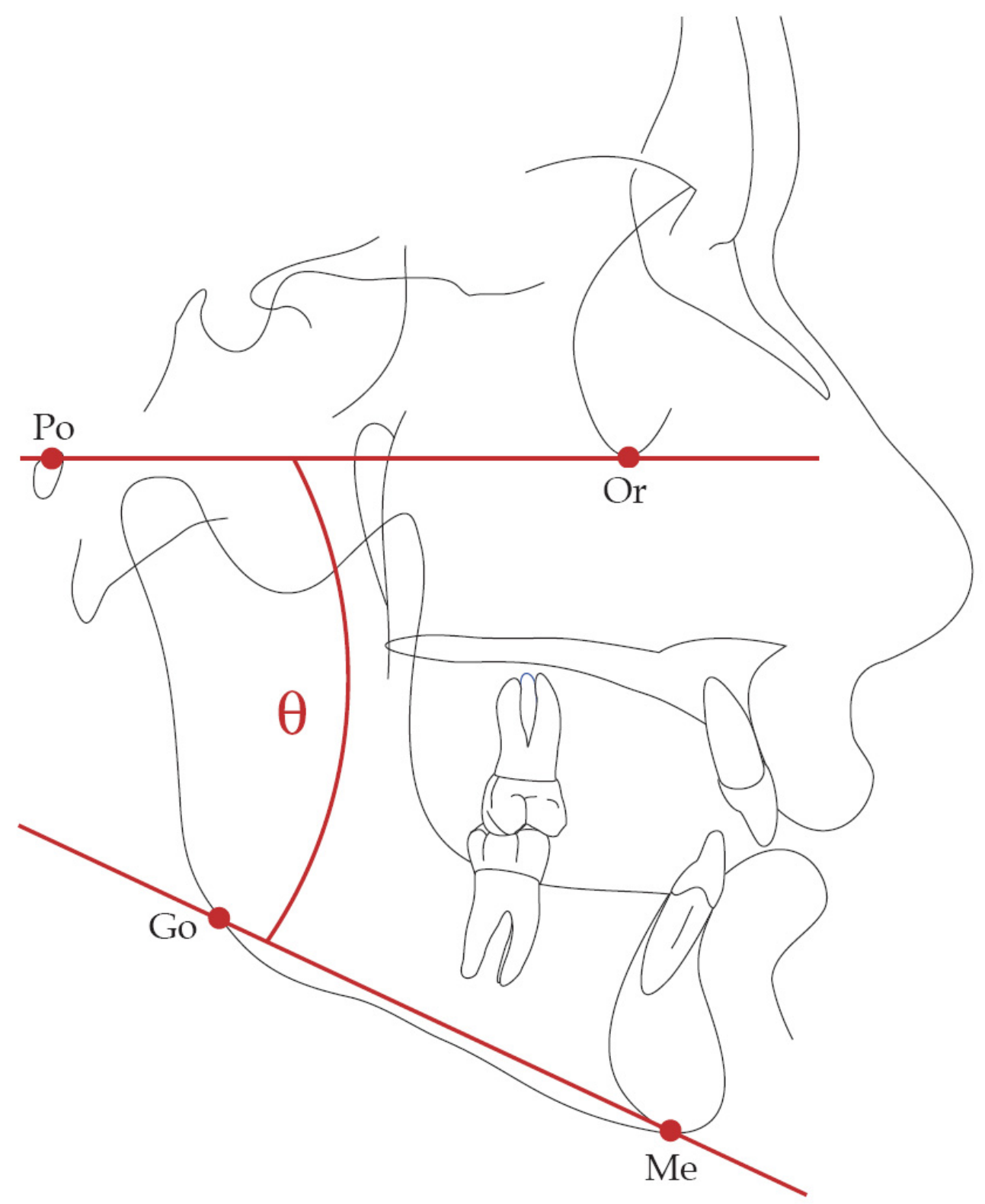

Figure A-5. Schematic tracing of a lateral cephalogram showing construction of FMA. This is the angle formed by the intersection of Frankfort Horizontal and the line through anatomic Gonion and Menton. 


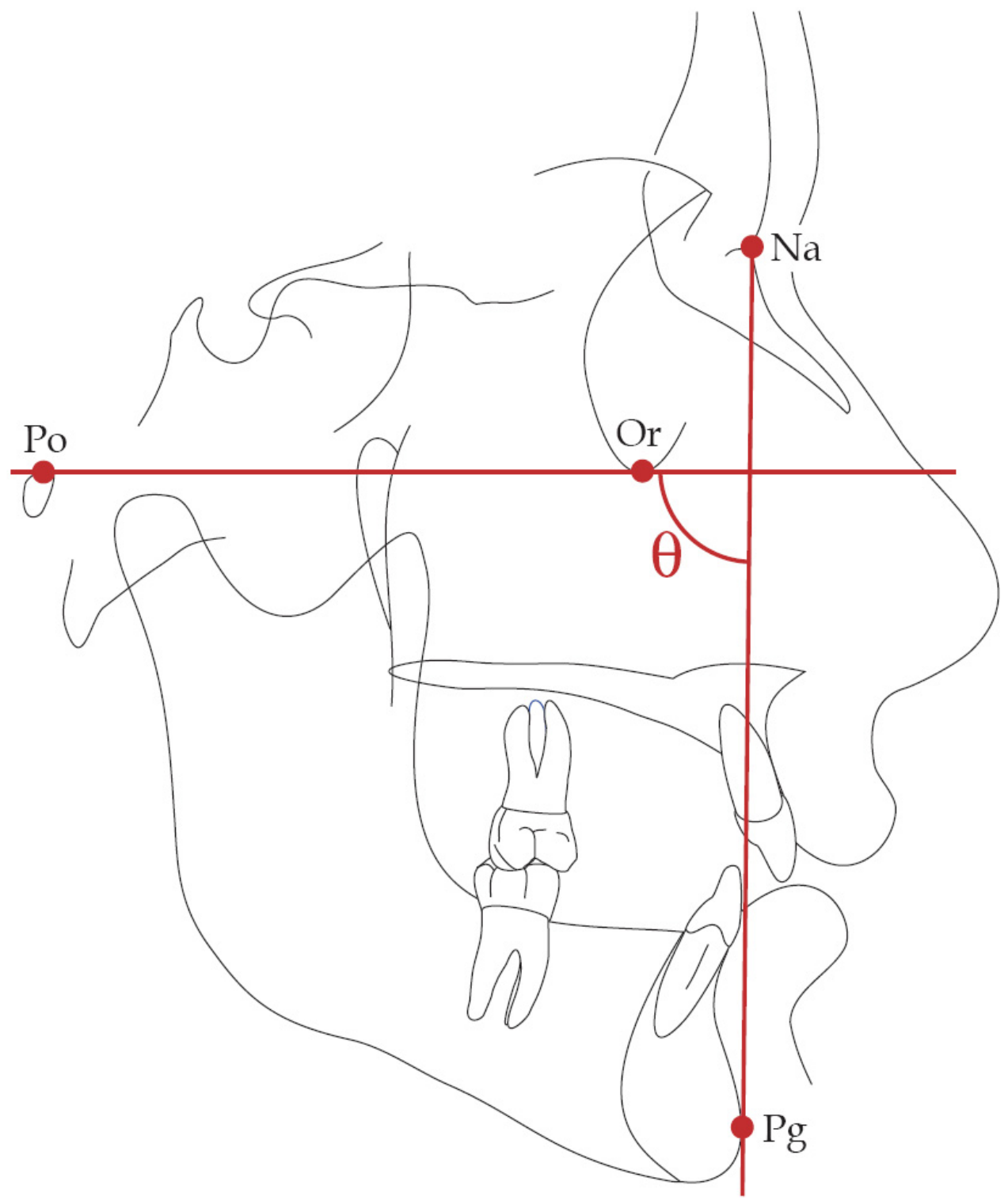

Figure A-6. Schematic tracing of a lateral cephalogram showing construction of Downs' facial angle. This is the angle formed by the posterior-inferior intersection of Frankfort Horizontal and Nasion-Pogonion lines. 


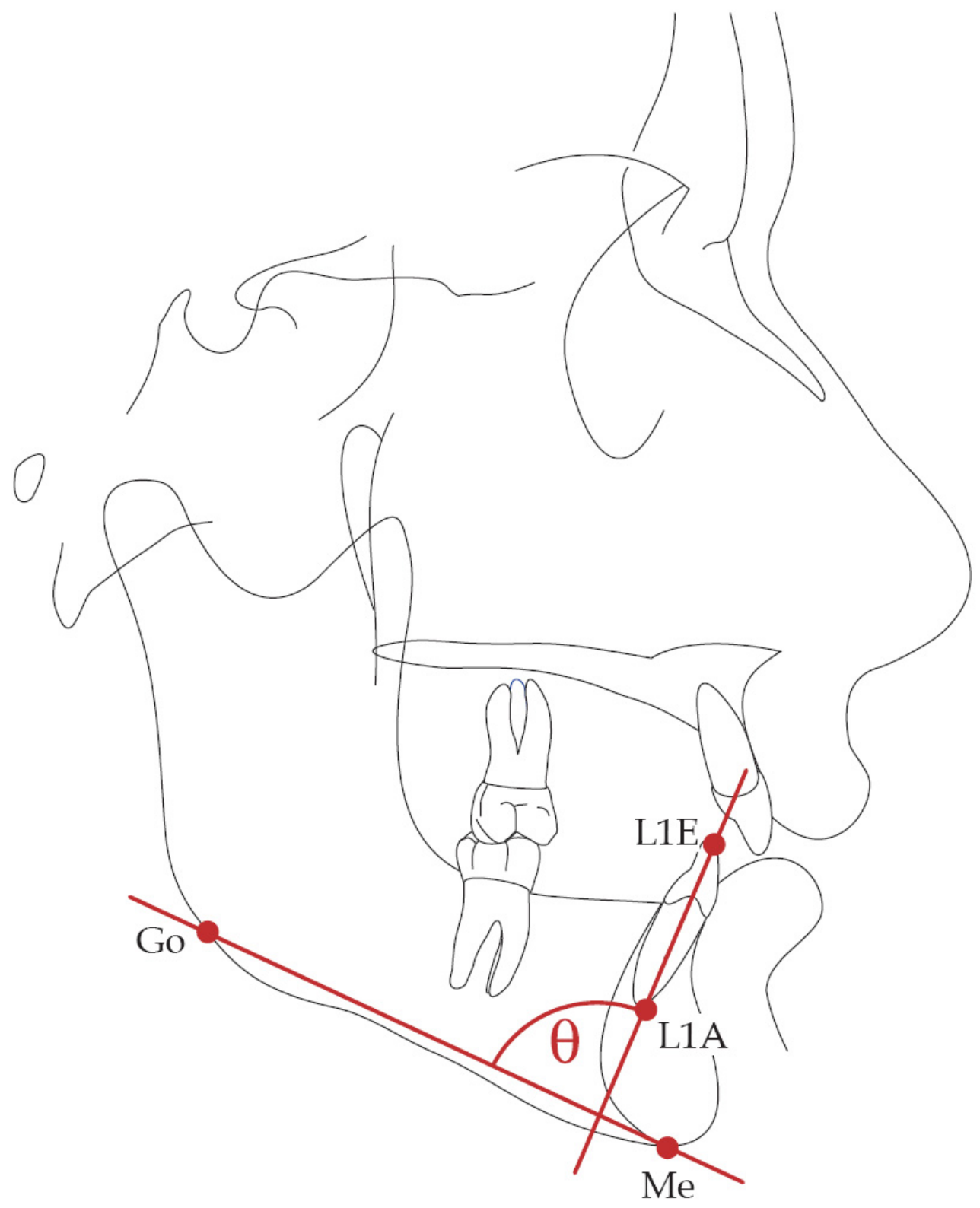

Figure A-7. Schematic tracing of a lateral cephalogram showing construction of the angle of the mandibular incisor and the mandibular plane (IMPA). 


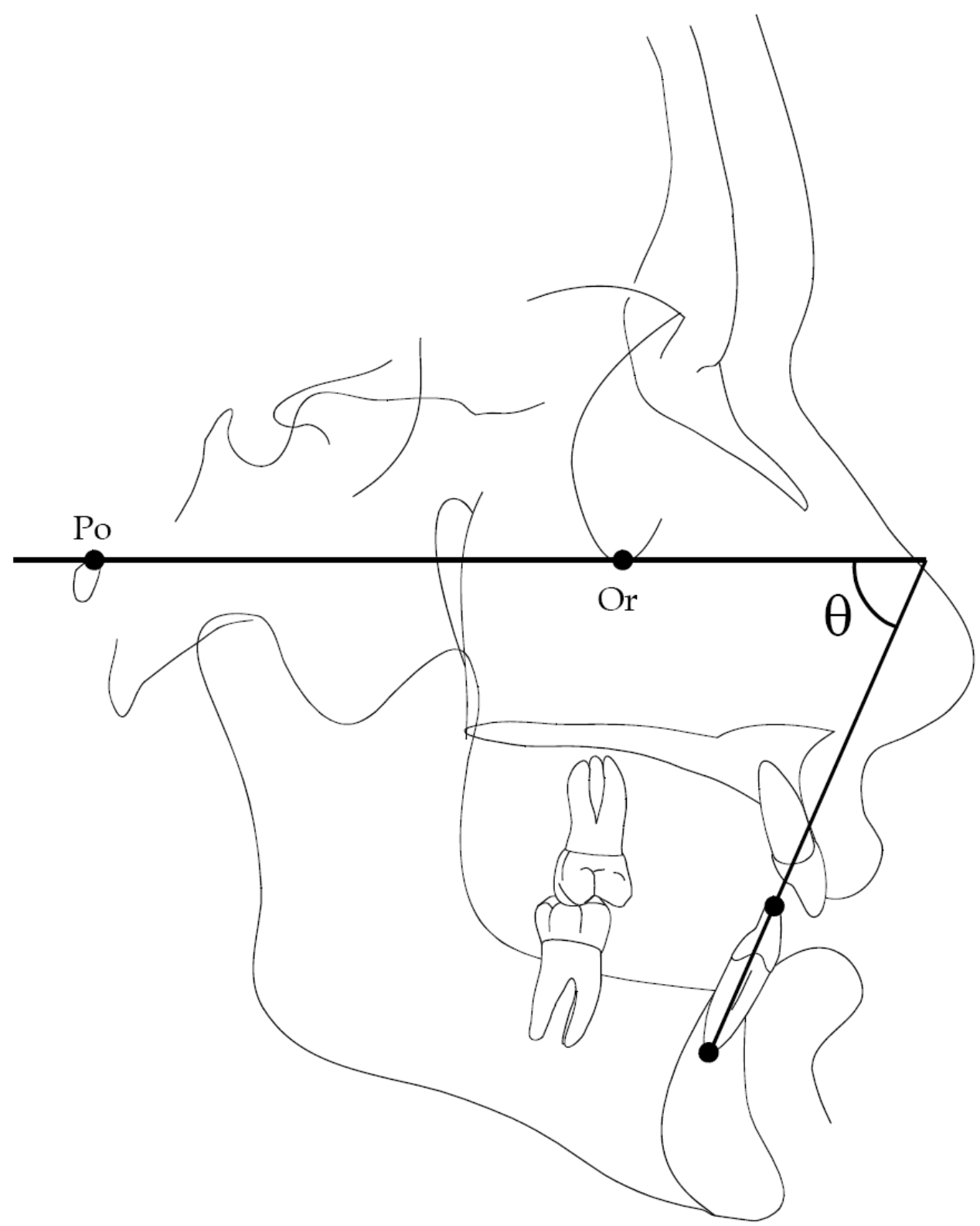

Figure A-8. Schematic tracing of a lateral cephalogram showing construction of FMIA measurement. This is formed by the intersection of Frankfort Horizontal and the long axis of the mandibular incisor (L1 apex and L1 incisal edge). 


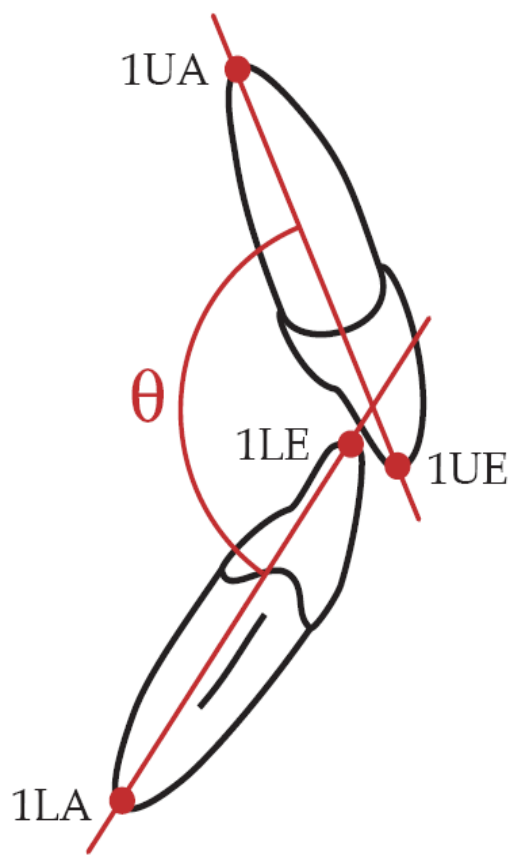

Figure A-9. Schematic tracing of a lateral cephalogram showing construction of the angle between the upper incisor with the lower incisor (i.e., interincisal angle). 


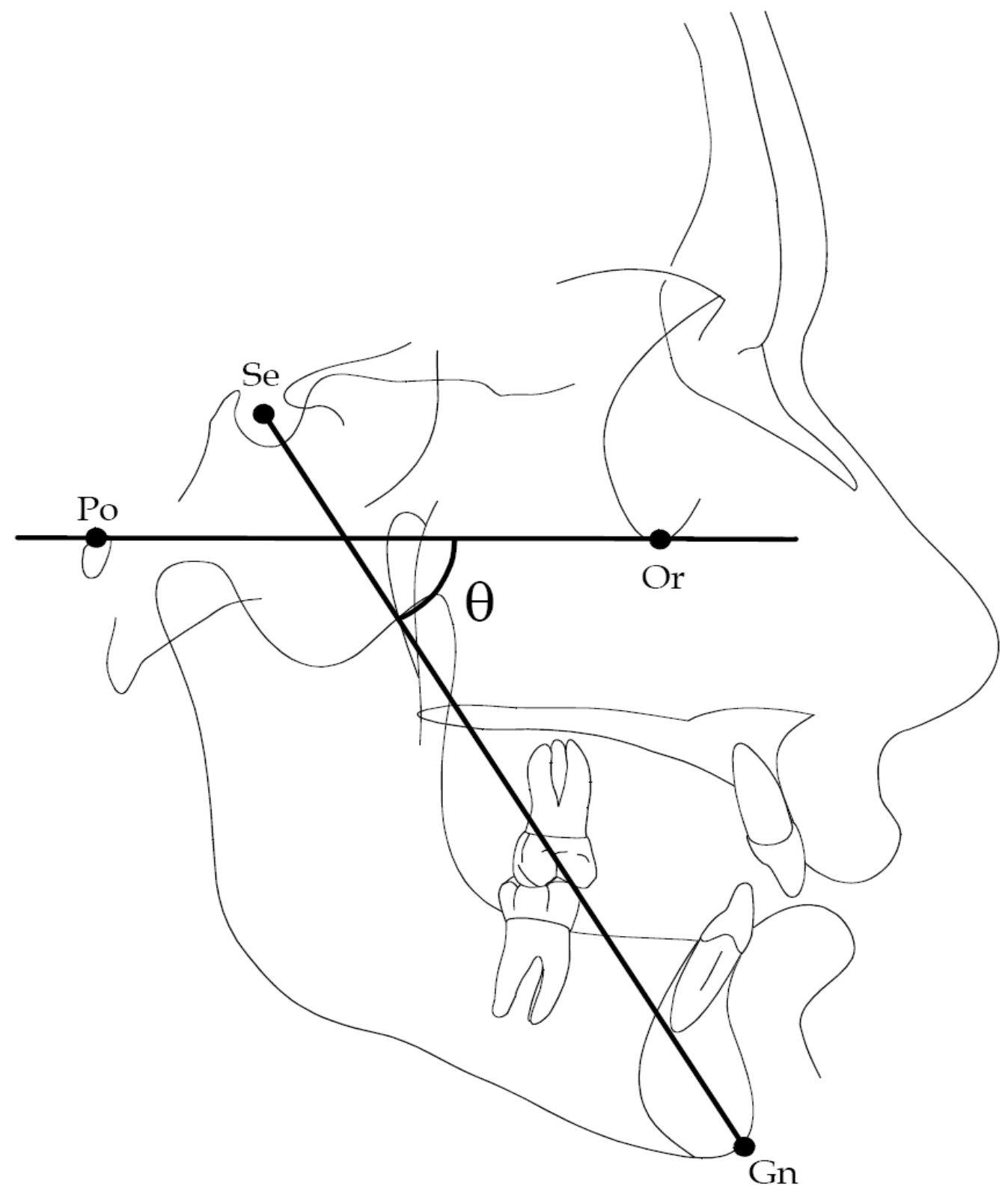

Figure A-10. Schematic tracing of a lateral cephalogram showing construction of the $\mathrm{Y}$-axis angle. This is the anterior-inferior angle at the intersection of Frankfort Horizontal and the Sella-Gnathion line. 


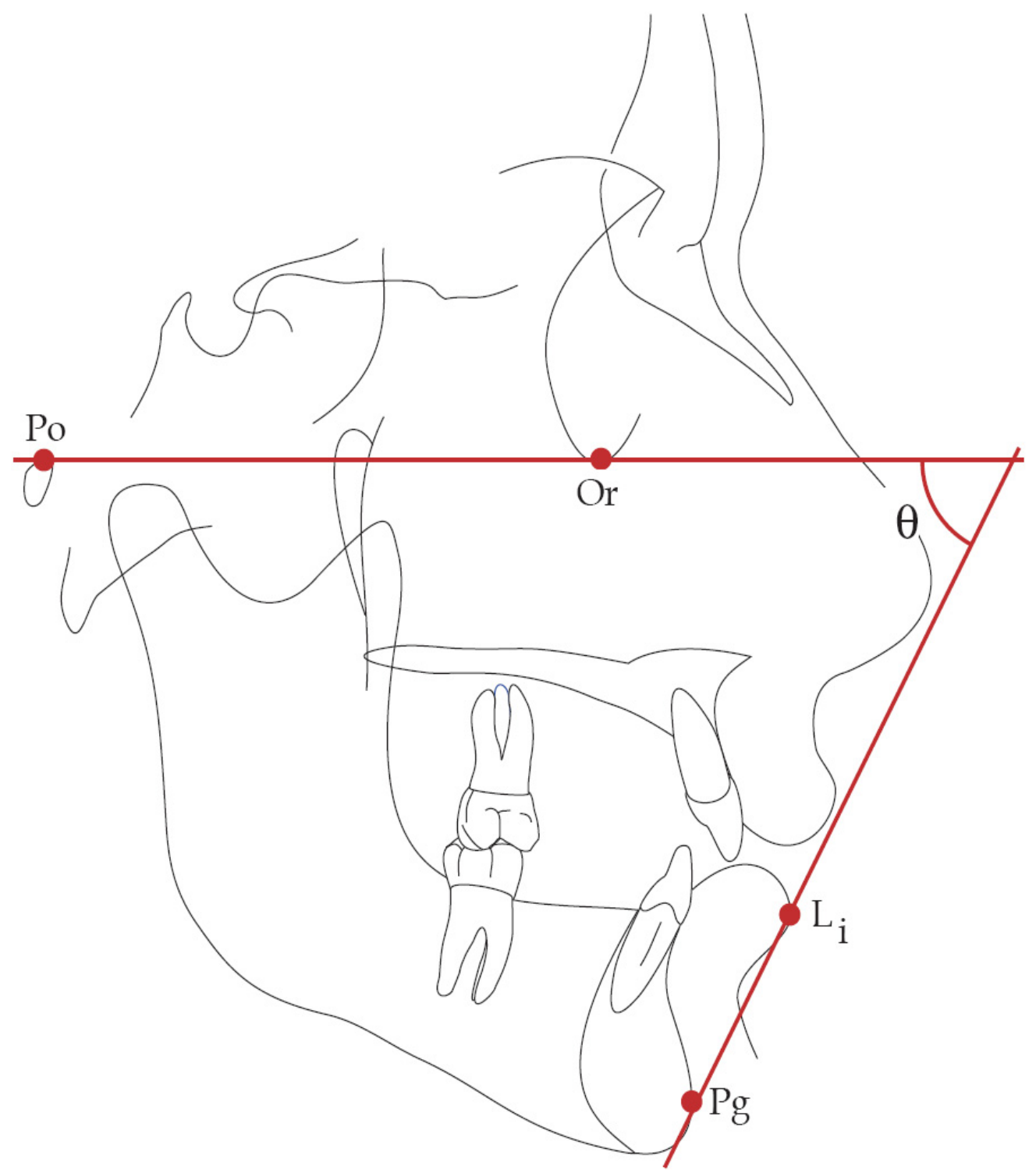

Figure A-11. Merrifield's Z angle, the inferior-posterior angle between Frankfort Horizontal (Porion and Orbitale) and Merrifield's profile line (a line drawn through the most prominent lip and the tangent of the soft tissue chin). In this diagram, both lips are equally prominent (and thus coincident along the line), and the diagram is drawn to Labrale inferius. 


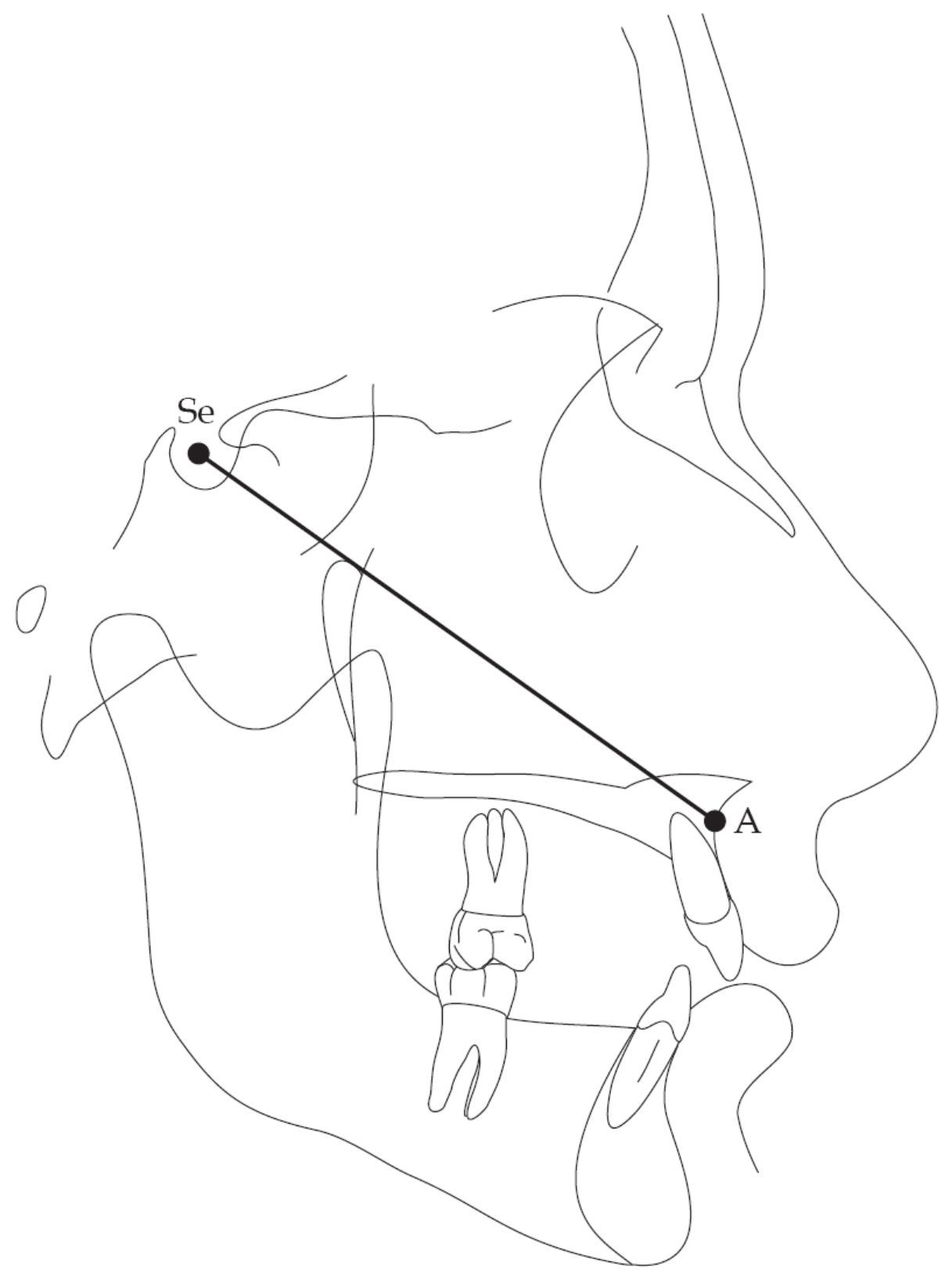

Figure A-12. Schematic tracing of a lateral cephalogram showing construction of Sella-A Point linear distance measurement. The distance between the two points is measured in millimeters. 


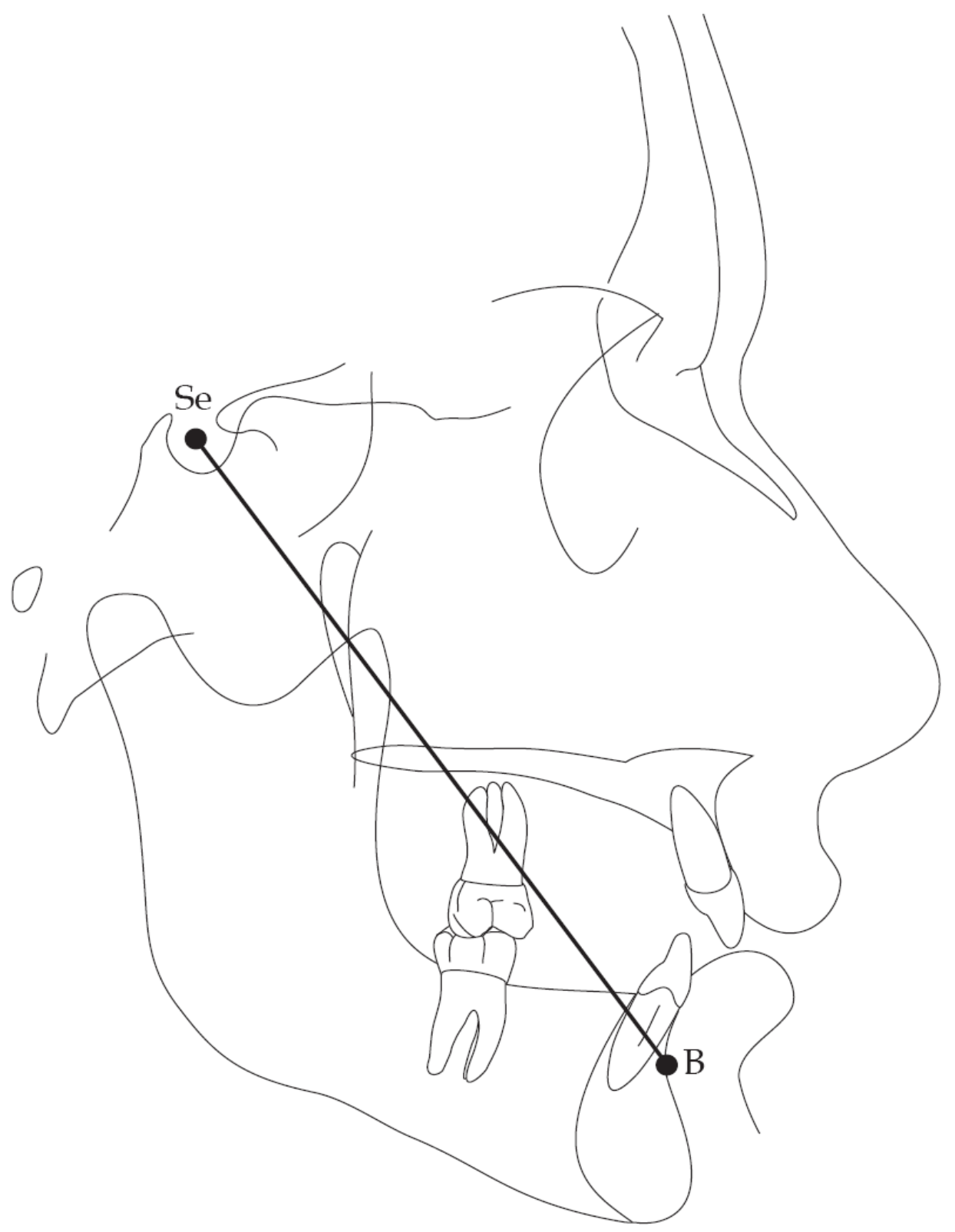

Figure A-13. Schematic tracing of a lateral cephalogram showing construction of Sella-B Point linear distance measurement. The distance between the two points is measured in millimeters. 


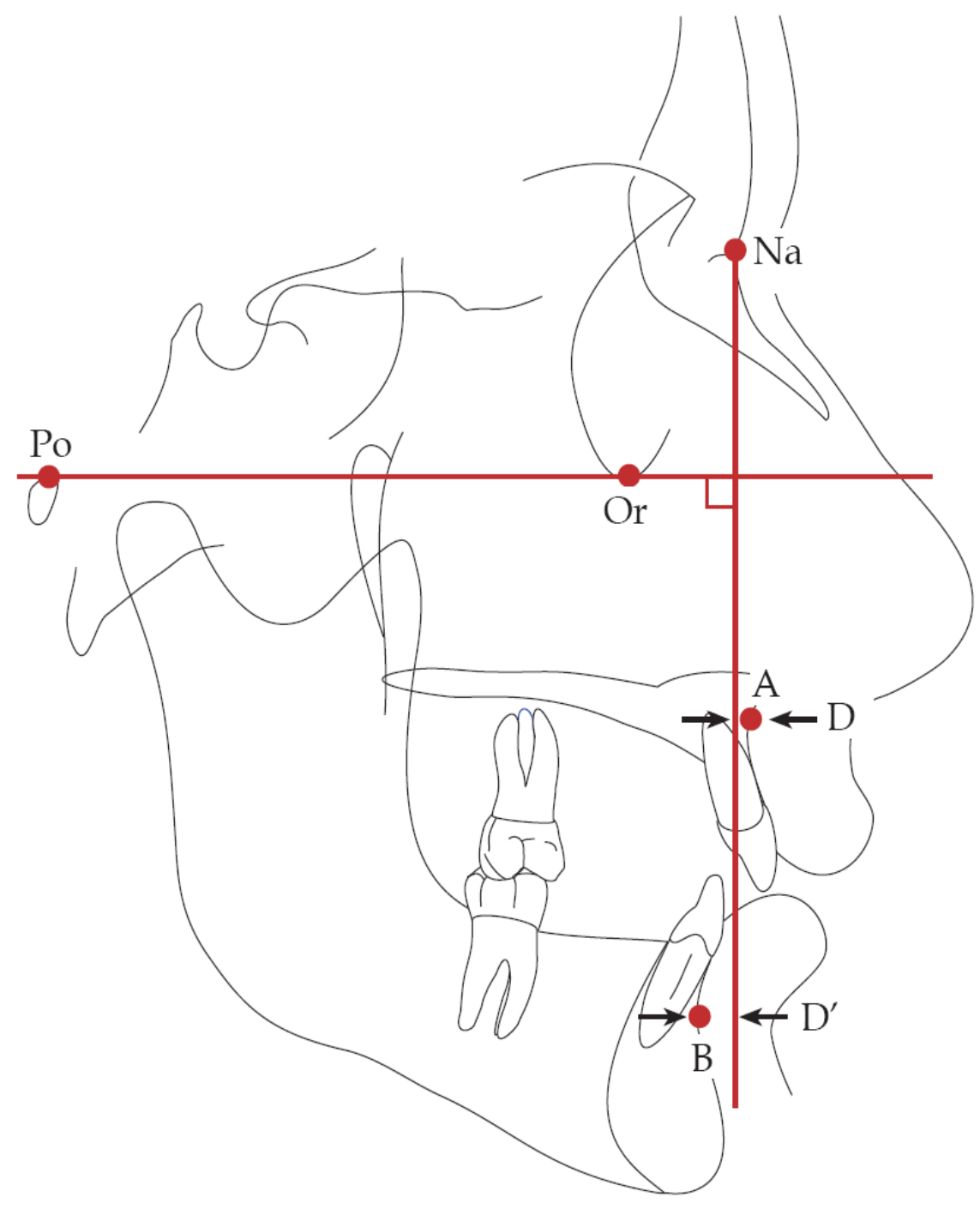

Figure A-14. Schematic tracing of a lateral cephalogram showing construction of the A Point to Nasion-Perpendicular (labeled D) and B Point to NasionPerpendicular (labeled $\mathrm{D}^{\prime}$ ) measurements. Both distances are measured parallel with Frankfort horizontal in millimeters. Values are negative when B Point is distal to Nasion-perpendicular. 


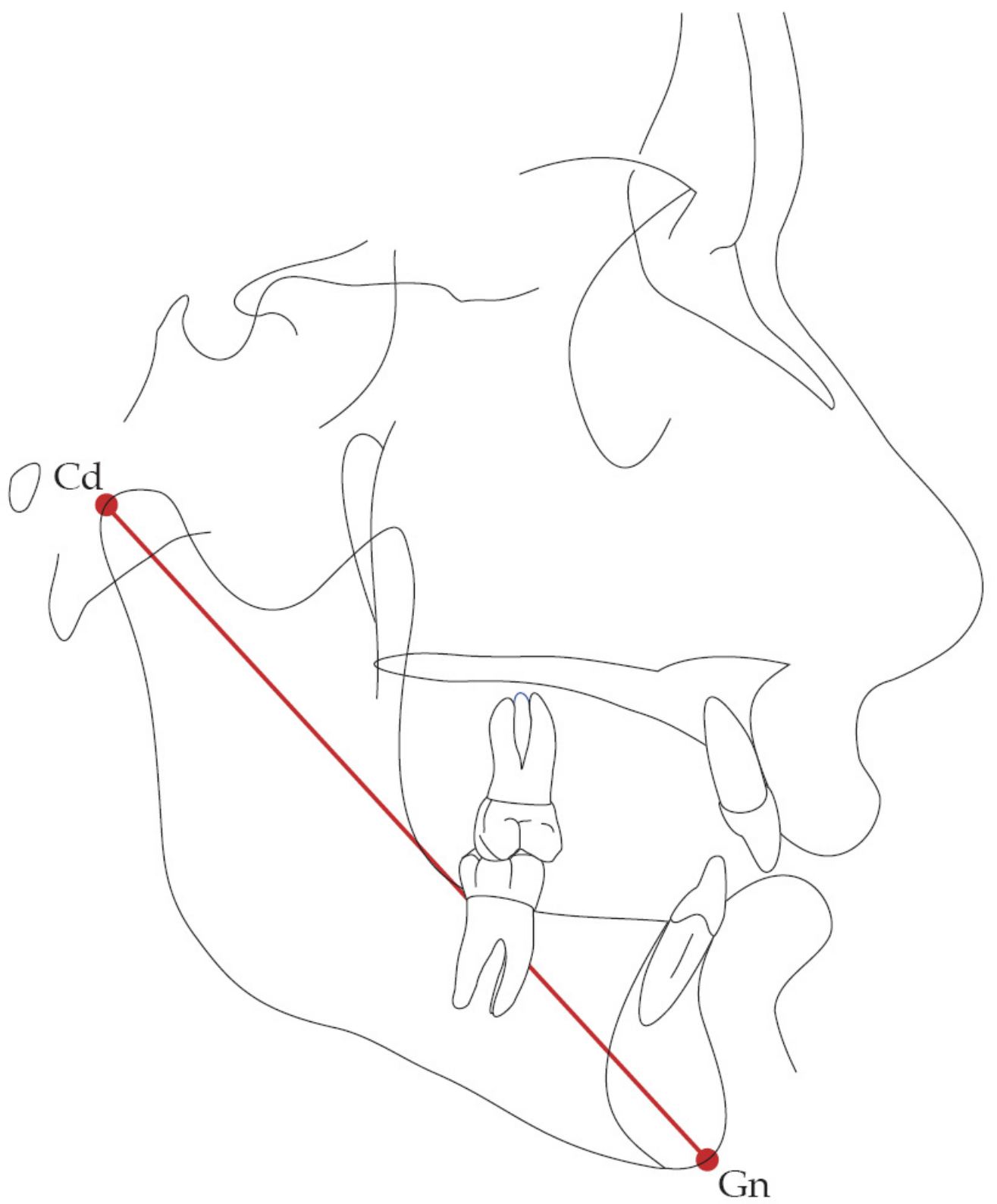

Figure A-15. Schematic tracing of a lateral cephalogram showing construction of the Condylion to Gnathion (also referred to as mandibular length). 

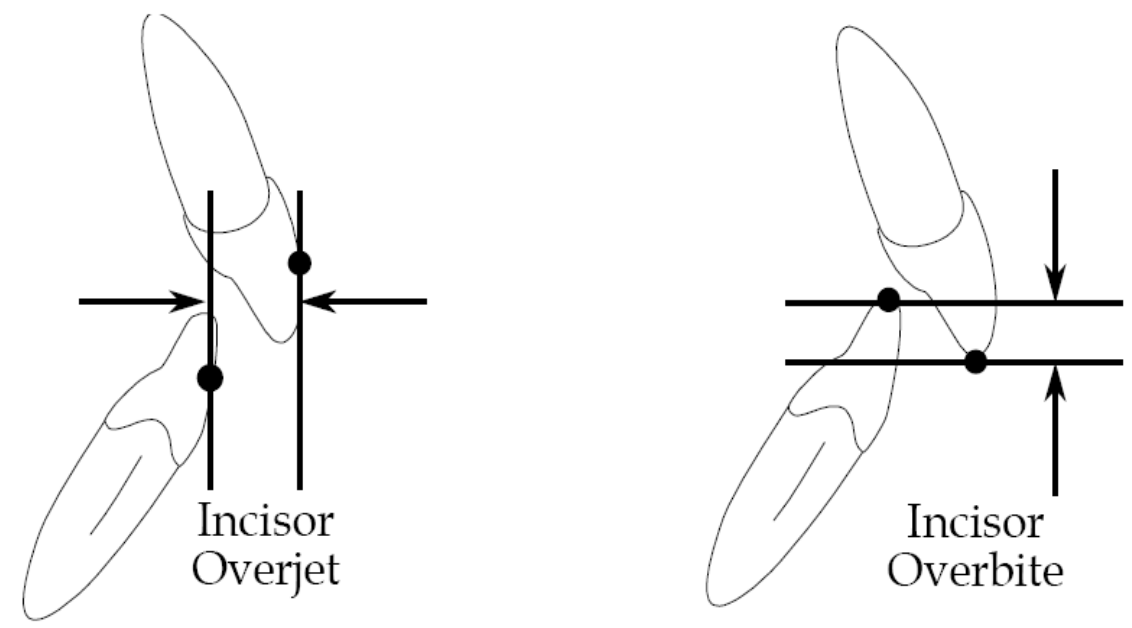

Figure A-16. Schematic diagram showing the overbite and overjet measurements. Distances are measured relative to Downs' occlusal plane. 


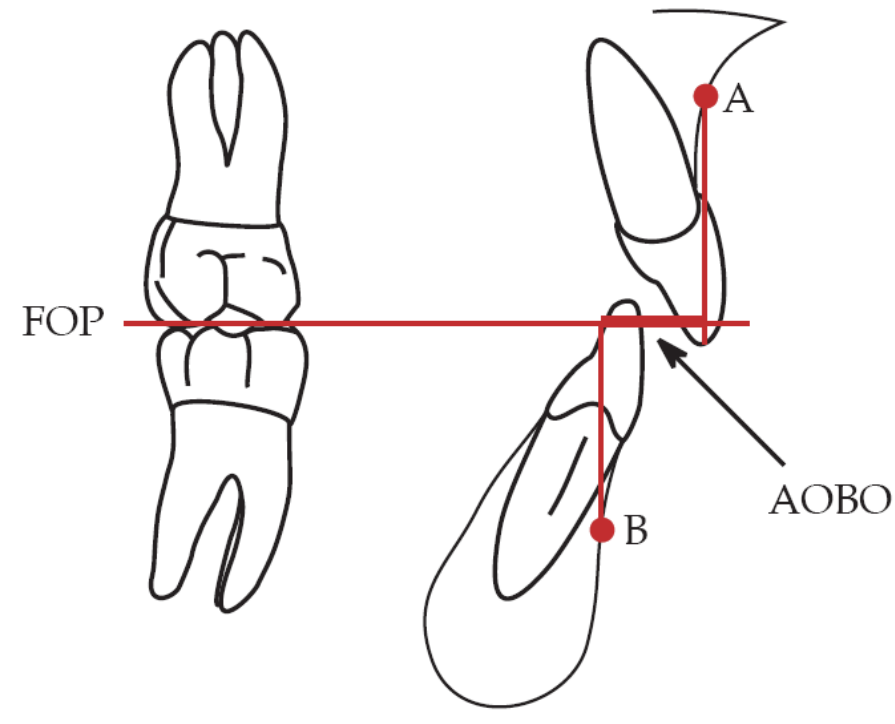

Figure A-17. Schematic of the method of measuring the AOBO discrepancy. Point A and Point $B$ are projected orthogonally onto the functional occlusal plane. AOBO is the millimetric distance between the projected line segment along the occlusal plane. 


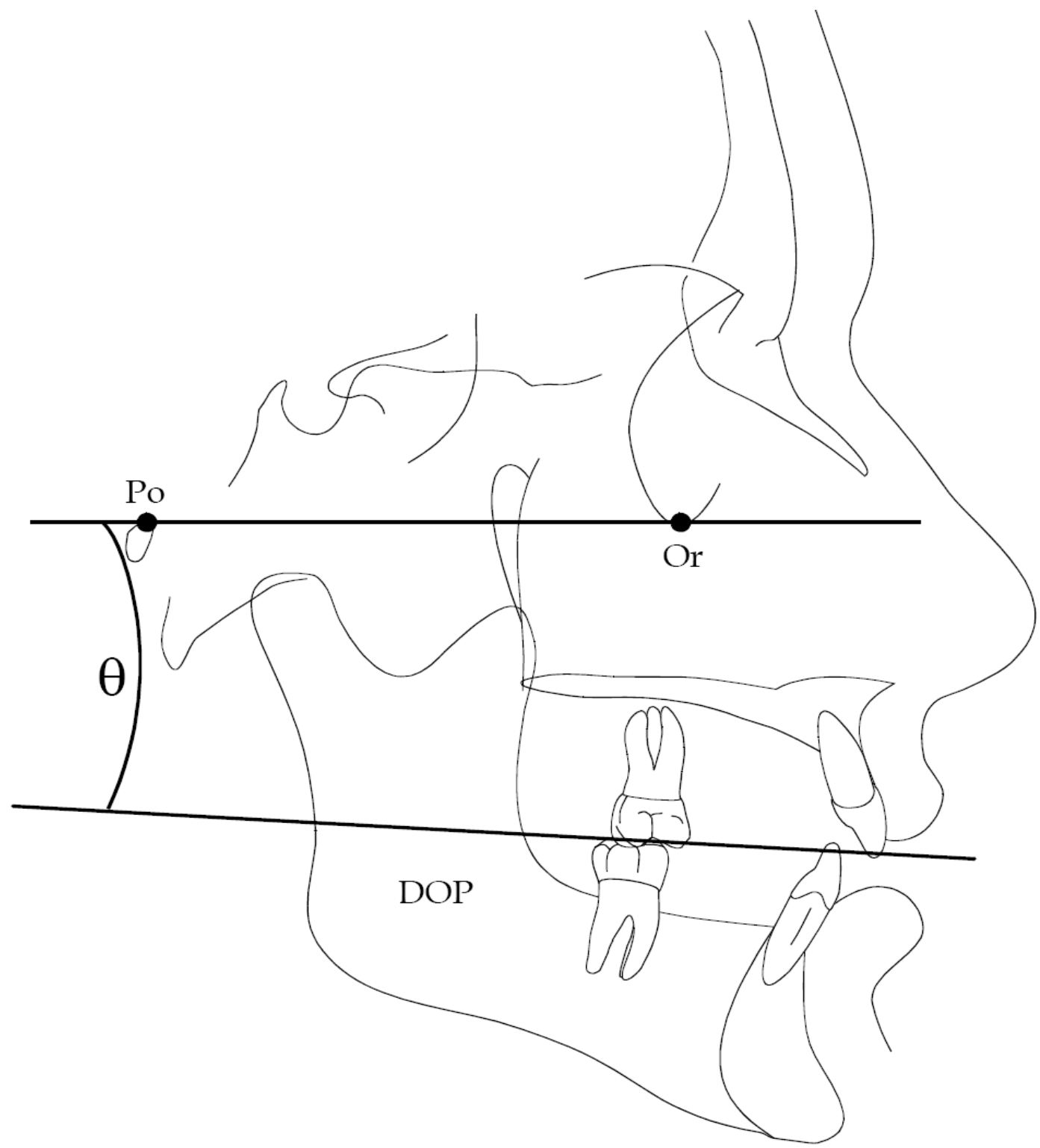

Figure A-18. Schematic tracing of a lateral cephalogram showing construction of the Downs' occlusal plane angle. This is the angle formed by the intersection of Frankfort Horizontal and Downs' occlusal plane. 
APPENDIX B.

DESCRIPTIVE STATISTICS, BY TREATMENT AND SEX, FOR THE CEPHALOMETRIC VARIABLES EVALUATED AT THE START OF TREATMENT 
Table B-1. Descriptive statistics at the pretreatment examination for the $\mathrm{Z}$ angle (degrees).

\begin{tabular}{lccccc}
\hline \multirow{2}{*}{ Variable } & \multicolumn{2}{c}{ MARA } & & \multicolumn{2}{c}{ Edgewise } \\
\cline { 2 - 3 } \cline { 5 - 6 } & Females & Males & & Females & Males \\
\hline Arithmetic mean & 70.31 & 70.15 & & 63.64 & 66.72 \\
Standard deviation & 8.16 & 8.04 & & 12.55 & 7.95 \\
Standard error mean & 1.92 & 1.58 & & 2.81 & 1.45 \\
Upper 95\% CL & 74.36 & 73.40 & 69.51 & 69.69 \\
Lower 95\% CL & 66.25 & 66.91 & 57.76 & 63.75 \\
Sample size & 18 & 26 & 20 & 30 \\
Variance & 66.58 & 64.59 & 157.48 & 63.19 \\
Skewness & 0.15 & 0.41 & 0.32 & 0.58 \\
Kurtosis & 0.31 & -0.99 & 0.27 & 0.01 \\
Number missing & 2 & 5 & 0 & 1 \\
\hline
\end{tabular}


Table B-2. Descriptive statistics at the pretreatment examination for the E Plane (mm).

\begin{tabular}{lccccc}
\hline \multirow{2}{*}{ Variable } & \multicolumn{2}{c}{ MARA } & & \multicolumn{2}{c}{ Edgewise } \\
\cline { 2 - 3 } \cline { 5 - 6 } & Females & Males & & Females & Males \\
\hline Arithmetic mean & -0.24 & -0.16 & & 1.48 & 0.53 \\
Standard deviation & 2.23 & 2.84 & & 3.16 & 2.32 \\
Standard error mean & 0.53 & 0.58 & & 0.71 & 0.42 \\
Upper 95\% CL & 0.87 & 1.04 & & 2.96 & 1.40 \\
Lower 95\% CL & -1.36 & -1.36 & & 0.00 & -0.34 \\
Sample size & 18 & 24 & 20 & 30 \\
Variance & 4.99 & 8.09 & 9.98 & 5.39 \\
Skewness & -0.54 & 0.10 & -0.39 & 0.27 \\
Kurtosis & -0.74 & 0.41 & 0.21 & -0.23 \\
Number missing & 2 & 7 & 0 & 1 \\
\hline
\end{tabular}


Table B-3. Descriptive statistics at the pretreatment examination for the Y-Axis Angle (degrees).

\begin{tabular}{lccccc}
\hline \multirow{2}{*}{ Variable } & \multicolumn{2}{c}{ MARA } & & \multicolumn{2}{c}{ Edgewise } \\
\cline { 2 - 3 } \cline { 5 - 6 } & Females & Males & & Females & Males \\
\hline Arithmetic mean & 69.35 & 68.69 & & 68.83 & 68.87 \\
Standard deviation & 3.40 & 3.53 & & 3.09 & 2.99 \\
Standard error mean & 0.76 & 0.63 & & 0.69 & 0.54 \\
Upper 95\% CL & 70.93 & 69.99 & & 70.27 & 69.97 \\
Lower 95\% CL & 67.76 & 67.40 & & 67.38 & 67.77 \\
Sample size & 20 & 31 & & 20 & 31 \\
Variance & 11.54 & 12.49 & & 9.56 & 8.95 \\
Skewness & -1.09 & 0.44 & 0.03 & -0.08 \\
Kurtosis & 1.69 & -0.68 & -0.32 & -1.00 \\
Number missing & 0 & 0 & 0 & 0 \\
\hline
\end{tabular}


Table B-4. Descriptive statistics at the pretreatment examination for the NAP Angle (degrees).

\begin{tabular}{lccccc}
\hline \multirow{2}{*}{ Variable } & \multicolumn{2}{c}{ MARA } & & \multicolumn{2}{c}{ Edgewise } \\
\cline { 2 - 3 } \cline { 5 - 6 } & Females & Males & & Females & Males \\
Arithmetic mean & 10.16 & 10.29 & & 11.59 & 9.96 \\
Standard deviation & 4.99 & 4.97 & & 5.86 & 4.32 \\
Standard error mean & 1.11 & 0.89 & & 1.31 & 0.78 \\
Upper 95\% CL & 12.49 & 12.11 & & 14.33 & 11.55 \\
Lower 95\% CL & 7.82 & 8.47 & & 8.85 & 8.38 \\
Sample size & 20 & 31 & 20 & 31 \\
Variance & 24.86 & 24.73 & 34.39 & 18.65 \\
Skewness & 0.84 & -0.05 & 0.08 & 0.26 \\
Kurtosis & 0.33 & -0.89 & -0.32 & 0.69 \\
Number missing & 0 & 0 & 0 & 0 \\
\hline
\end{tabular}


Table B-5. Descriptive statistics at the pretreatment examination for the SNA Angle (degrees).

\begin{tabular}{lccccc}
\hline \multirow{2}{*}{ Variable } & \multicolumn{2}{c}{ MARA } & & \multicolumn{2}{c}{ Edgewise } \\
\cline { 2 - 3 } \cline { 5 - 6 } & Females & Males & & Females & Males \\
\hline Arithmetic mean & 81.14 & 81.32 & & 82.39 & 81.24 \\
Standard deviation & 4.12 & 3.80 & & 2.77 & 3.79 \\
Standard error mean & 0.92 & 0.68 & & 0.62 & 0.68 \\
Upper 95\% CL & 83.06 & 82.71 & & 83.69 & 82.63 \\
Lower 95\% CL & 79.21 & 79.93 & & 81.09 & 79.84 \\
Sample size & 20 & 31 & & 20 & 31 \\
Variance & 16.95 & 14.41 & & 7.69 & 14.39 \\
Skewness & 0.81 & 0.25 & -0.67 & 0.25 \\
Kurtosis & 0.62 & -0.93 & -0.89 & -0.14 \\
Number missing & 0 & 0 & 0 & 0 \\
\hline
\end{tabular}


Table B-6. Descriptive statistics at the pretreatment examination for the SNB Angle (degrees).

\begin{tabular}{lccccc}
\hline \multirow{2}{*}{ Variable } & \multicolumn{2}{c}{ MARA } & & \multicolumn{2}{c}{ Edgewise } \\
\cline { 2 - 3 } \cline { 5 - 6 } & Females & Males & & Females & Males \\
\hline Arithmetic mean & 75.78 & 75.61 & & 76.25 & 75.79 \\
Standard deviation & 3.61 & 3.50 & & 2.60 & 3.08 \\
Standard error mean & 0.81 & 0.63 & & 0.58 & 0.55 \\
Upper 95\% CL & 77.47 & 76.89 & & 77.46 & 76.92 \\
Lower 95\% CL & 74.09 & 74.33 & & 75.03 & 74.66 \\
Sample size & 20 & 31 & & 20 & 31 \\
Variance & 13.03 & 12.26 & 6.74 & 9.51 \\
Skewness & 0.94 & -0.38 & -0.08 & -0.17 \\
Kurtosis & 1.02 & -0.82 & -1.10 & -0.79 \\
Number missing & 0 & 0 & 0 & 0 \\
\hline
\end{tabular}


Table B-7. Descriptive statistics at the pretreatment examination for the ANB Angle (degrees).

\begin{tabular}{lccccc}
\hline \multirow{2}{*}{ Variable } & \multicolumn{2}{c}{ MARA } & & \multicolumn{2}{c}{ Edgewise } \\
\cline { 2 - 3 } \cline { 5 - 6 } & Females & Males & & Females & Males \\
\hline Arithmetic mean & 5.33 & 5.73 & & 6.14 & 5.45 \\
Standard deviation & 1.76 & 1.60 & & 2.31 & 1.87 \\
Standard error mean & 0.39 & 0.29 & & 0.52 & 0.34 \\
Upper 95\% CL & 6.15 & 6.31 & & 7.22 & 6.14 \\
Lower 95\% CL & 4.50 & 5.14 & & 5.06 & 4.76 \\
Sample size & 20 & 31 & & 20 & 31 \\
Variance & 3.11 & 2.55 & & 5.34 & 3.51 \\
Skewness & 0.72 & -0.16 & 0.47 & 0.92 \\
Kurtosis & 0.40 & -0.81 & 0.12 & 1.70 \\
Number missing & 0 & 0 & 0 & 0 \\
\hline
\end{tabular}


Table B-8. Descriptive statistics at the pretreatment examination for the AOBO (mm).

\begin{tabular}{lccccc}
\hline \multirow{2}{*}{ Variable } & \multicolumn{2}{c}{ MARA } & & \multicolumn{2}{c}{ Edgewise } \\
\cline { 2 - 3 } \cline { 5 - 6 } & Females & Males & & Females & Males \\
\hline Arithmetic mean & 2.76 & 3.52 & & 3.56 & 3.35 \\
Standard deviation & 2.08 & 1.92 & & 2.69 & 3.24 \\
Standard error mean & 0.46 & 0.34 & & 0.60 & 0.58 \\
Upper 95\% CL & 3.73 & 4.22 & & 4.82 & 4.54 \\
Lower 95\% CL & 1.79 & 2.81 & & 2.30 & 2.17 \\
Sample size & 20 & 31 & & 20 & 31 \\
Variance & 4.32 & 3.69 & & 7.26 & 10.51 \\
Skewness & -1.13 & 0.17 & 0.03 & 0.53 \\
Kurtosis & 1.50 & -0.25 & -0.11 & -0.13 \\
Number missing & 0 & 0 & 0 & 0 \\
\hline
\end{tabular}


Table B-9. Descriptive statistics at the pretreatment examination for the FMA Angle (degrees).

\begin{tabular}{lccccc}
\hline \multirow{2}{*}{ Variable } & \multicolumn{2}{c}{ MARA } & & \multicolumn{2}{c}{ Edgewise } \\
\cline { 2 - 3 } \cline { 5 - 6 } & Females & Males & & Females & Males \\
\hline Arithmetic mean & 25.33 & 24.35 & & 26.90 & 25.94 \\
Standard deviation & 5.76 & 4.61 & & 5.59 & 4.00 \\
Standard error mean & 1.29 & 0.83 & & 1.25 & 0.72 \\
Upper 95\% CL & 28.03 & 26.04 & & 29.51 & 27.41 \\
Lower 95\% CL & 22.63 & 22.65 & & 24.28 & 24.48 \\
Sample size & 20 & 31 & 20 & 31 \\
Variance & 33.16 & 21.24 & 31.22 & 15.96 \\
Skewness & -0.39 & -0.01 & -0.51 & -0.64 \\
Kurtosis & -0.98 & -0.97 & 0.21 & 0.13 \\
Number missing & 0 & 0 & 0 & 0 \\
\hline
\end{tabular}


Table B-10. Descriptive statistics at the pretreatment examination for the IMPA (degrees).

\begin{tabular}{lccccc}
\hline \multirow{2}{*}{ Variable } & \multicolumn{2}{c}{ MARA } & & \multicolumn{2}{c}{ Edgewise } \\
\cline { 2 - 3 } \cline { 5 - 6 } & Females & Males & & Females & Males \\
\hline Arithmetic mean & 93.43 & 97.75 & & 95.15 & 95.35 \\
Standard deviation & 5.64 & 7.20 & & 7.63 & 6.86 \\
Standard error mean & 1.26 & 1.29 & & 1.71 & 1.23 \\
Upper 95\% CL & 96.07 & 100.39 & & 98.72 & 97.87 \\
Lower 95\% CL & 90.79 & 95.11 & & 91.58 & 92.84 \\
Sample size & 20 & 31 & & 20 & 31 \\
Variance & 31.80 & 51.88 & & 58.29 & 47.04 \\
Skewness & -0.54 & -0.33 & 0.67 & -0.44 \\
Kurtosis & 0.33 & -0.80 & -0.03 & 0.09 \\
Number missing & 0 & 0 & 0 & 0 \\
\hline
\end{tabular}


Table B-11. Descriptive statistics at the pretreatment examination for the FMIA (degrees).

\begin{tabular}{lccccc}
\hline \multirow{2}{*}{ Variable } & \multicolumn{2}{c}{ MARA } & & \multicolumn{2}{c}{ Edgewise } \\
\cline { 2 - 3 } \cline { 5 - 6 } & Females & Males & & Females & Males \\
\hline Arithmetic mean & 61.26 & 57.91 & & 57.95 & 58.81 \\
Standard deviation & 6.19 & 7.88 & & 8.45 & 6.06 \\
Standard error mean & 1.38 & 1.42 & & 1.89 & 1.09 \\
Upper 95\% CL & 64.15 & 60.80 & & 61.90 & 61.03 \\
Lower 95\% CL & 58.36 & 55.02 & & 53.99 & 56.59 \\
Sample size & 20 & 31 & & 20 & 31 \\
Variance & 38.26 & 62.08 & & 71.37 & 36.69 \\
Skewness & 0.42 & -0.08 & -0.54 & 0.37 \\
Kurtosis & -0.51 & -0.79 & -0.51 & -0.14 \\
Number missing & 0 & 0 & 0 & 0 \\
\hline
\end{tabular}


Table B-12. Descriptive statistics at the pretreatment examination for the OP to FH Angle (degrees).

\begin{tabular}{lccccc}
\hline \multirow{2}{*}{ Variable } & \multicolumn{2}{c}{ MARA } & & \multicolumn{2}{c}{ Edgewise } \\
\cline { 2 - 3 } \cline { 5 - 6 } & Females & Males & & Females & Males \\
\hline Arithmetic mean & 8.76 & 8.74 & & 10.00 & 9.44 \\
Standard deviation & 4.61 & 3.59 & & 3.70 & 3.28 \\
Standard error mean & 1.03 & 0.65 & & 0.83 & 0.59 \\
Upper 95\% CL & 10.91 & 10.06 & & 11.73 & 10.64 \\
Lower 95\% CL & 6.60 & 7.42 & & 8.27 & 8.24 \\
Sample size & 20 & 31 & & 20 & 31 \\
Variance & 21.26 & 12.90 & & 13.65 & 10.73 \\
Skewness & -0.13 & -0.15 & -0.78 & -0.25 \\
Kurtosis & -1.15 & -0.84 & 0.28 & 0.09 \\
Number missing & 0 & 0 & 0 & 0 \\
\hline
\end{tabular}


Table B-13. Descriptive statistics at the pretreatment examination for the U1 to SeNa Angle (degrees).

\begin{tabular}{lccccc}
\hline \multirow{2}{*}{ Variable } & \multicolumn{2}{c}{ MARA } & & \multicolumn{2}{c}{ Edgewise } \\
\cline { 2 - 3 } \cline { 5 - 6 } & Females & Males & & Females & Males \\
\hline Arithmetic mean & 101.88 & 102.44 & & 103.26 & 108.92 \\
Standard deviation & 8.41 & 7.93 & & 9.19 & 20.28 \\
Standard error mean & 1.88 & 1.42 & & 2.05 & 3.64 \\
Upper 95\% CL & 105.81 & 105.35 & & 107.55 & 116.35 \\
Lower 95\% CL & 97.94 & 99.53 & & 98.96 & 101.48 \\
Sample size & 20 & 31 & & 20 & 31 \\
Variance & 70.75 & 62.94 & 84.41 & 411.24 \\
Skewness & -0.54 & 0.80 & -0.77 & 4.17 \\
Kurtosis & 0.06 & 1.02 & 0.66 & 20.58 \\
Number missing & 0 & 0 & 0 & 0 \\
\hline
\end{tabular}


Table B-14. Descriptive statistics at the pretreatment examination for the Interincisal Angle (degrees).

\begin{tabular}{lccccc}
\hline \multirow{2}{*}{ Variable } & \multicolumn{2}{c}{ MARA } & & \multicolumn{2}{c}{ Edgewise } \\
\cline { 2 - 3 } \cline { 5 - 6 } & Females & Males & & Females & Males \\
\hline Arithmetic mean & 129.71 & 126.19 & & 127.32 & 125.18 \\
Standard deviation & 10.47 & 12.46 & & 14.92 & 9.76 \\
Standard error mean & 2.34 & 2.24 & & 3.34 & 1.75 \\
Upper 95\% CL & 134.60 & 130.76 & & 134.30 & 128.76 \\
Lower 95\% CL & 124.81 & 121.62 & 120.33 & 121.61 \\
Sample size & 20 & 31 & 20 & 31 \\
Variance & 109.55 & 155.30 & 222.68 & 95.19 \\
Skewness & 0.69 & -0.42 & 0.26 & 1.44 \\
Kurtosis & -0.18 & -0.07 & 0.02 & 3.57 \\
Number missing & 0 & 0 & 0 & 0 \\
\hline
\end{tabular}


Table B-15. Descriptive statistics at the pretreatment examination for the U1 to $\mathrm{Na}$ A Pt Angle (degrees).

\begin{tabular}{lccccc}
\hline \multirow{2}{*}{ Variable } & \multicolumn{2}{c}{ MARA } & & \multicolumn{2}{c}{ Edgewise } \\
\cline { 2 - 3 } \cline { 5 - 6 } & Females & Males & & Females & Males \\
\hline Arithmetic mean & 20.75 & 21.12 & & 20.88 & 24.28 \\
Standard deviation & 8.79 & 8.41 & & 8.54 & 8.52 \\
Standard error mean & 1.97 & 1.51 & & 1.91 & 1.53 \\
Upper 95\% CL & 24.86 & 24.21 & & 24.88 & 27.41 \\
Lower 95\% CL & 16.63 & 18.04 & & 16.88 & 21.16 \\
Sample size & 20 & 31 & 20 & 31 \\
Variance & 77.30 & 70.71 & 73.01 & 72.59 \\
Skewness & 0.31 & 0.54 & -1.13 & 0.43 \\
Kurtosis & -0.23 & 0.17 & 1.13 & 0.86 \\
Number missing & 0 & 0 & 0 & 0 \\
\hline
\end{tabular}


Table B-16. Descriptive statistics at the pretreatment examination for the U1 to $\mathrm{Na}$ A Pt (mm).

\begin{tabular}{lccccc}
\hline \multirow{2}{*}{ Variable } & \multicolumn{2}{c}{ MARA } & & \multicolumn{2}{c}{ Edgewise } \\
\cline { 2 - 3 } \cline { 5 - 6 } & & & & & \\
Arithmetes & Males & & & \\
Standard deviation & 3.03 & 2.57 & & 3.18 & 3.28 \\
Standard error mean & 0.68 & 0.46 & & 0.71 & 0.59 \\
Upper 95\% CL & 3.92 & 3.57 & & 4.16 & 5.57 \\
Lower 95\% CL & 1.07 & 1.68 & & 1.19 & 3.17 \\
Sample size & 20 & 31 & & 20 & 31 \\
Variance & 9.21 & 6.62 & 10.12 & 10.73 \\
Skewness & -0.06 & 0.40 & -1.29 & 0.27 \\
Kurtosis & -1.03 & -0.38 & 1.20 & 0.13 \\
Number missing & 0 & 0 & 0 & 0 \\
\hline
\end{tabular}


Table B-17. Descriptive statistics at the pretreatment examination for the L1 to A Pg Angle (degrees).

\begin{tabular}{lccccc}
\hline \multirow{2}{*}{ Variable } & \multicolumn{2}{c}{ MARA } & & \multicolumn{2}{c}{ Edgewise } \\
\cline { 2 - 3 } \cline { 5 - 6 } & & & & & \\
& & & & & \\
Arithmales & Males & & & \\
Standard mean & 19.18 & 22.41 & & 20.28 & 20.66 \\
Standard error mean & 0.78 & 1.16 & & 1.65 & 1.05 \\
Upper 95\% CL & 20.82 & 24.79 & & 23.73 & 22.80 \\
Lower 95\% CL & 17.53 & 20.03 & & 16.83 & 18.52 \\
Sample size & 20 & 31 & & 20 & 31 \\
Variance & 12.32 & 42.07 & 54.31 & 34.07 \\
Skewness & -0.42 & -0.72 & 0.47 & -0.51 \\
Kurtosis & 0.42 & 1.09 & -0.39 & 0.38 \\
Number missing & 0 & 0 & 0 & 0 \\
\hline
\end{tabular}


Table B-18. Descriptive statistics at the pretreatment examination for the L1 to A $\operatorname{Pg}(\mathrm{mm})$.

\begin{tabular}{lccccc}
\hline \multirow{2}{*}{ Variable } & \multicolumn{2}{c}{ MARA } & & \multicolumn{2}{c}{ Edgewise } \\
\cline { 2 - 3 } \cline { 5 - 6 } & & & & & \\
Arithmetes & Males & & & \\
Standard mean & -0.04 & 0.50 & & 0.75 & 0.46 \\
Standard error mean & 0.40 & 0.51 & & 0.65 & 0.37 \\
Upper 95\% CL & 0.80 & 1.53 & & 2.11 & 1.23 \\
Lower 95\% CL & -0.88 & -0.54 & & -0.61 & -0.30 \\
Sample size & 20 & 31 & & 20 & 31 \\
Variance & 3.24 & 7.95 & & 8.49 & 4.33 \\
Skewness & 0.42 & 0.29 & 0.07 & -0.35 \\
Kurtosis & 1.12 & 0.41 & -0.37 & -0.18 \\
Number missing & 0 & 0 & 0 & 0 \\
\hline
\end{tabular}


Table B-19. Descriptive statistics at the pretreatment examination for the L1 to Na B Pt (degrees).

\begin{tabular}{lccccc}
\hline \multirow{2}{*}{ Variable } & \multicolumn{2}{c}{ MARA } & & \multicolumn{2}{c}{ Edgewise } \\
\cline { 2 - 3 } \cline { 5 - 6 } & & & & & \\
Arithmetes & Males & & & \\
Standard deviation & 4.15 & 7.68 & & 3.05 & 2.58 \\
Standard error mean & 0.93 & 1.38 & & 0.68 & 0.46 \\
Upper 95\% CL & 26.17 & 29.77 & & 6.27 & 5.45 \\
Lower 95\% CL & 22.28 & 24.13 & & 3.42 & 3.56 \\
Sample size & 20 & 31 & & 20 & 31 \\
Variance & 17.23 & 59.00 & & 9.33 & 6.68 \\
Skewness & -0.35 & 0.11 & 0.82 & 0.49 \\
Kurtosis & -0.40 & -0.22 & 1.40 & 0.90 \\
Number missing & 0 & 0 & 0 & 0 \\
\hline
\end{tabular}


Table B-20. Descriptive statistics at the pretreatment examination for the L1 to Na B Pt (degrees).

\begin{tabular}{lccccc}
\hline \multirow{2}{*}{ Variable } & \multicolumn{2}{c}{ MARA } & & \multicolumn{2}{c}{ Edgewise } \\
\cline { 2 - 3 } \cline { 5 - 6 } & Females & Males & & Females & Males \\
\hline Arithmetic mean & 3.60 & 4.74 & & 25.68 & 25.07 \\
Standard deviation & 1.48 & 3.08 & & 7.89 & 6.25 \\
Standard error mean & 0.33 & 0.55 & & 1.76 & 1.12 \\
Upper 95\% CL & 4.29 & 5.87 & & 29.37 & 27.36 \\
Lower 95\% CL & 2.90 & 3.61 & & 21.99 & 22.78 \\
Sample size & 20 & 31 & & 20 & 31 \\
Variance & 2.18 & 9.47 & 62.24 & 39.01 \\
Skewness & -0.30 & 0.03 & 0.82 & -0.58 \\
Kurtosis & -0.77 & 0.15 & 0.71 & -0.05 \\
Number missing & 0 & 0 & 0 & 0 \\
\hline
\end{tabular}


Table B-21. Descriptive statistics at the pretreatment examination for the Facial Angle (degrees).

\begin{tabular}{lccccc}
\hline \multirow{2}{*}{ Variable } & \multicolumn{2}{c}{ MARA } & & \multicolumn{2}{c}{ Edgewise } \\
\cline { 2 - 3 } \cline { 5 - 6 } & Females & Males & & Females & Males \\
\hline Arithmetic mean & 85.98 & 85.75 & & 84.21 & 84.68 \\
Standard deviation & 3.64 & 3.30 & & 2.64 & 2.83 \\
Standard error mean & 0.81 & 0.59 & & 0.59 & 0.51 \\
Upper 95\% CL & 87.68 & 86.96 & & 85.45 & 85.72 \\
Lower 95\% CL & 84.28 & 84.53 & & 82.97 & 83.64 \\
Sample size & 20 & 31 & 20 & 31 \\
Variance & 13.25 & 10.92 & 6.97 & 8.03 \\
Skewness & 0.53 & 0.46 & 0.05 & -0.61 \\
Kurtosis & -0.17 & 0.57 & -0.46 & -0.11 \\
Number missing & 0 & 0 & 0 & 0 \\
\hline
\end{tabular}


Table B-22. Descriptive statistics at the pretreatment examination for the Mandibular length (mm).

\begin{tabular}{lccccc}
\hline \multirow{2}{*}{ Variable } & \multicolumn{2}{c}{ MARA } & & \multicolumn{2}{c}{ Edgewise } \\
\cline { 2 - 3 } \cline { 5 - 6 } & Females & Males & & Females & Males \\
\hline Arithmetic mean & 109.70 & 112.48 & & 109.80 & 109.93 \\
Standard deviation & 6.72 & 6.87 & 6.07 & 7.91 \\
Standard error mean & 1.50 & 1.23 & & 1.36 & 1.42 \\
Upper 95\% CL & 112.84 & 115.00 & & 112.64 & 112.83 \\
Lower 95\% CL & 106.56 & 109.96 & 106.95 & 107.03 \\
Sample size & 20 & 31 & 20 & 31 \\
Variance & 45.15 & 47.14 & 36.88 & 62.53 \\
Skewness & -0.23 & 0.44 & 0.51 & 0.47 \\
Kurtosis & 1.68 & -0.33 & -0.91 & 1.52 \\
Number missing & 0 & 0 & 0 & 0 \\
\hline
\end{tabular}


Table B-23. Descriptive statistics at the pretreatment examination for the Sella-toA Point (mm).

\begin{tabular}{lccccc}
\hline \multirow{2}{*}{ Variable } & \multicolumn{2}{c}{ MARA } & & \multicolumn{2}{c}{ Edgewise } \\
\cline { 2 - 3 } \cline { 5 - 6 } & Females & Males & & Females & Males \\
\hline Arithmetic mean & 85.53 & 87.24 & & 85.27 & 85.25 \\
Standard deviation & 3.51 & 4.19 & & 4.86 & 5.53 \\
Standard error mean & 0.78 & 0.75 & & 1.09 & 0.99 \\
Upper 95\% CL & 87.17 & 88.78 & & 87.54 & 87.28 \\
Lower 95\% CL & 83.88 & 85.70 & & 82.99 & 83.22 \\
Sample size & 20 & 31 & 20 & 31 \\
Variance & 12.31 & 17.56 & 23.59 & 30.56 \\
Skewness & -0.05 & 0.20 & -0.74 & 0.42 \\
Kurtosis & -0.38 & -0.52 & 0.33 & 1.82 \\
Number missing & 0 & 0 & 0 & 0 \\
\hline
\end{tabular}


Table B-24. Descriptive statistics at the pretreatment examination for the Sella-toB Point (mm).

\begin{tabular}{lccccc}
\hline \multirow{2}{*}{ Variable } & \multicolumn{2}{c}{ MARA } & & \multicolumn{2}{c}{ Edgewise } \\
\cline { 2 - 3 } \cline { 5 - 6 } & Females & Males & & Females & Males \\
\hline Arithmetic mean & 103.98 & 105.53 & & 103.85 & 104.75 \\
Standard deviation & 5.17 & 5.13 & 6.85 & 7.27 \\
Standard error mean & 1.16 & 0.92 & & 1.53 & 1.31 \\
Upper 95\% CL & 106.40 & 107.41 & & 107.05 & 107.42 \\
Lower 95\% CL & 101.55 & 103.65 & 100.64 & 102.09 \\
Sample size & 20 & 31 & 20 & 31 \\
Variance & 26.75 & 26.31 & 46.95 & 52.86 \\
Skewness & 0.13 & 0.22 & -0.90 & -0.44 \\
Kurtosis & -0.30 & -0.71 & 1.79 & 1.14 \\
Number missing & 0 & 0 & 0 & 0 \\
\hline
\end{tabular}


Table B-25. Descriptive statistics at the pretreatment examination for the Overbite (mm).

\begin{tabular}{lccccc}
\hline \multirow{2}{*}{ Variable } & \multicolumn{2}{c}{ MARA } & & \multicolumn{2}{c}{ Edgewise } \\
\cline { 2 - 3 } \cline { 5 - 6 } & & & & & \\
Arithmetes & Males & & & \\
Semales & Males \\
Standard deviation & 1.91 & 1.96 & & 2.54 & 1.74 \\
Standard error mean & 0.43 & 0.35 & & 0.57 & 0.31 \\
Upper 95\% CL & 4.70 & 4.56 & & 4.25 & 3.82 \\
Lower 95\% CL & 2.92 & 3.12 & & 1.88 & 2.54 \\
Sample size & 20 & 31 & & 20 & 31 \\
Variance & 3.63 & 3.84 & 6.46 & 3.04 \\
Skewness & 0.22 & -0.44 & -0.22 & -0.25 \\
Kurtosis & 0.46 & -0.14 & -0.85 & 0.06 \\
Number missing & 0 & 0 & 0 & 0 \\
\hline
\end{tabular}


Table B-26. Descriptive statistics at the pretreatment examination for the Overjet (mm).

\begin{tabular}{lccccc}
\hline \multirow{2}{*}{ Variable } & \multicolumn{2}{c}{ MARA } & & \multicolumn{2}{c}{ Edgewise } \\
\cline { 2 - 3 } \cline { 5 - 6 } & Females & Males & & Females & Males \\
\hline Arithmetic mean & 6.59 & 6.16 & & 6.64 & 8.17 \\
Standard deviation & 2.07 & 1.94 & & 2.68 & 2.89 \\
Standard error mean & 0.46 & 0.35 & & 0.60 & 0.52 \\
Upper 95\% CL & 7.56 & 6.87 & & 7.89 & 9.23 \\
Lower 95\% CL & 5.61 & 5.45 & & 5.39 & 7.11 \\
Sample size & 20 & 31 & 20 & 31 \\
Variance & 4.30 & 3.77 & 7.18 & 8.35 \\
Skewness & 0.63 & 0.43 & 0.37 & 0.46 \\
Kurtosis & -0.62 & -0.31 & -0.65 & -0.55 \\
Number missing & 0 & 0 & 0 & 0 \\
\hline
\end{tabular}


Table B-27. Descriptive statistics at the pretreatment examination for the A Pt to Na-Perp (mm).

\begin{tabular}{lccccc}
\hline \multirow{2}{*}{ Variable } & \multicolumn{2}{c}{ MARA } & & \multicolumn{2}{c}{ Edgewise } \\
\cline { 2 - 3 } \cline { 5 - 6 } & Females & Males & & Females & Males \\
\hline Arithmetic mean & 0.81 & 0.60 & & -0.25 & -0.60 \\
Standard deviation & 4.03 & 3.95 & & 3.03 & 3.96 \\
Standard error mean & 0.90 & 0.71 & & 0.68 & 0.71 \\
Upper 95\% CL & 2.69 & 2.05 & & 1.17 & 0.85 \\
Lower 95\% CL & -1.07 & -0.85 & & -1.66 & -2.05 \\
Sample size & 20 & 31 & 20 & 31 \\
Variance & 16.21 & 15.63 & 9.20 & 15.65 \\
Skewness & 0.68 & 0.64 & 0.81 & -0.32 \\
Kurtosis & 0.24 & 1.14 & 0.94 & -0.23 \\
Number missing & 0 & 0 & 0 & 0 \\
\hline
\end{tabular}


Table B-28. Descriptive statistics at the pretreatment examination for the B Pt to Na-Perp (mm).

\begin{tabular}{lccccc}
\hline \multirow{2}{*}{ Variable } & \multicolumn{2}{c}{ MARA } & & \multicolumn{2}{c}{ Edgewise } \\
\cline { 2 - 3 } \cline { 5 - 6 } & Females & Males & & Females & Males \\
\hline Arithmetic mean & -7.47 & -8.56 & & -10.56 & -10.33 \\
Standard deviation & 5.69 & 5.31 & & 4.21 & 4.86 \\
Standard error mean & 1.27 & 0.95 & & 0.94 & 0.87 \\
Upper 95\% CL & -4.80 & -6.61 & & -8.58 & -8.55 \\
Lower 95\% CL & -10.13 & -10.51 & -12.53 & -12.11 \\
Sample size & 20 & 31 & & 20 & 31 \\
Variance & 32.34 & 28.23 & 17.75 & 23.64 \\
Skewness & 0.43 & 0.50 & 0.20 & -0.23 \\
Kurtosis & -0.36 & 0.93 & 0.70 & -0.62 \\
Number missing & 0 & 0 & 0 & 0 \\
\hline
\end{tabular}


APPENDIX C.

DESCRIPTIVE STATISTICS, BY TREATMENT AND SEX, FOR THE CEPHALOMETRIC VARIABLES EVALUATED AT THE END OF TREATMENT 
Table C-1. Descriptive statistics at the posttreatment examination for the Z Angle (degrees).

\begin{tabular}{lccccc}
\hline \multirow{2}{*}{ Variable } & \multicolumn{2}{c}{ MARA } & & \multicolumn{2}{c}{ Edgewise } \\
\cline { 2 - 3 } \cline { 5 - 6 } & Females & Males & & Females & Males \\
\hline Arithmetic mean & 75.47 & 71.36 & & 71.01 & 74.87 \\
Standard deviation & 7.99 & 7.79 & & 9.01 & 7.56 \\
Standard error mean & 1.83 & 1.47 & & 2.07 & 1.36 \\
Upper 95\% CL & 79.32 & 74.38 & & 75.35 & 77.64 \\
Lower 95\% CL & 71.62 & 68.34 & & 66.66 & 72.09 \\
Sample size & 19 & 28 & & 19 & 31 \\
Variance & 63.80 & 60.61 & 81.18 & 57.14 \\
Skewness & -0.27 & 0.18 & 0.16 & 0.51 \\
Kurtosis & -0.61 & -0.95 & -0.97 & -0.41 \\
Number missing & 1 & 3 & 1 & 0 \\
\hline
\end{tabular}


Table C-2. Descriptive statistics at the posttreatment examination for the E Plane (mm).

\begin{tabular}{lccccc}
\hline \multirow{2}{*}{ Variable } & \multicolumn{2}{c}{ MARA } & & \multicolumn{2}{c}{ Edgewise } \\
\cline { 2 - 3 } \cline { 5 - 6 } & Females & Males & & Females & Males \\
\hline Arithmetic mean & -2.02 & -1.01 & & -0.94 & -2.46 \\
Standard deviation & 1.75 & 2.07 & & 2.24 & 2.22 \\
Standard error mean & 0.40 & 0.42 & & 0.51 & 0.40 \\
Upper 95\% CL & -1.17 & -0.14 & & 0.14 & -1.65 \\
Lower 95\% CL & -2.86 & -1.89 & -2.02 & -3.28 \\
Sample size & 19 & 24 & & 19 & 31 \\
Variance & 3.06 & 4.28 & & 5.03 & 4.93 \\
Skewness & -0.67 & -0.81 & -0.17 & -0.32 \\
Kurtosis & 0.05 & 0.13 & -0.68 & -0.66 \\
Number missing & 1 & 7 & 1 & 0 \\
\hline
\end{tabular}


Table C-3. Descriptive statistics at the posttreatment examination for the Y-Axis Angle (degrees).

\begin{tabular}{lccccc}
\hline \multirow{2}{*}{ Variable } & \multicolumn{2}{c}{ MARA } & & \multicolumn{2}{c}{ Edgewise } \\
\cline { 2 - 3 } \cline { 5 - 6 } & Females & Males & & Females & Males \\
\hline Arithmetic mean & 69.73 & 69.03 & & 68.95 & 69.24 \\
Standard deviation & 3.87 & 3.88 & & 3.49 & 3.23 \\
Standard error mean & 0.87 & 0.71 & & 0.78 & 0.58 \\
Upper 95\% CL & 71.54 & 70.48 & & 70.58 & 70.42 \\
Lower 95\% CL & 67.92 & 67.58 & & 67.32 & 68.05 \\
Sample size & 20 & 30 & & 20 & 31 \\
Variance & 15.00 & 15.07 & & 12.16 & 10.44 \\
Skewness & -0.83 & 0.40 & 0.04 & 0.15 \\
Kurtosis & 1.71 & -0.74 & -0.98 & -0.47 \\
Number missing & 0 & 1 & 0 & 0 \\
\hline
\end{tabular}


Table C-4. Descriptive statistics at the posttreatment examination for the NAP Angle (degrees).

\begin{tabular}{lccccc}
\hline \multirow{2}{*}{ Variable } & \multicolumn{2}{c}{ MARA } & & \multicolumn{2}{c}{ Edgewise } \\
\cline { 2 - 3 } \cline { 5 - 6 } & Females & Males & & Females & Males \\
\hline Arithmetic mean & 5.14 & 3.83 & & 5.43 & 5.61 \\
Standard deviation & 4.40 & 5.04 & & 5.85 & 5.09 \\
Standard error mean & 0.98 & 0.90 & & 1.31 & 0.91 \\
Upper 95\% CL & 7.20 & 5.68 & & 8.17 & 7.48 \\
Lower 95\% CL & 3.08 & 1.98 & & 2.68 & 3.74 \\
Sample size & 20 & 31 & & 20 & 31 \\
Variance & 19.36 & 25.39 & 34.28 & 25.95 \\
Skewness & 0.20 & -0.12 & 0.17 & -0.28 \\
Kurtosis & -0.70 & -0.83 & -0.96 & 0.54 \\
Number missing & 0 & 0 & 0 & 0 \\
\hline
\end{tabular}


Table C-5. Descriptive statistics at the posttreatment examination for the SNA Angle (degrees).

\begin{tabular}{lccccc}
\hline \multirow{2}{*}{ Variable } & \multicolumn{2}{c}{ MARA } & & \multicolumn{2}{c}{ Edgewise } \\
\cline { 2 - 3 } \cline { 5 - 6 } & Females & Males & & Females & Males \\
Arithmetic mean & 79.61 & 79.61 & & 80.11 & 79.95 \\
Standard deviation & 4.13 & 4.22 & & 3.06 & 3.82 \\
Standard error mean & 0.92 & 0.76 & & 0.68 & 0.69 \\
Upper 95\% CL & 81.54 & 81.16 & & 81.54 & 81.35 \\
Lower 95\% CL & 77.68 & 78.06 & & 78.67 & 78.55 \\
Sample size & 20 & 31 & & 20 & 31 \\
Variance & 17.06 & 17.84 & 9.36 & 14.57 \\
Skewness & 0.44 & 0.34 & -0.11 & -0.03 \\
Kurtosis & 0.40 & -0.53 & -0.39 & 0.66 \\
Number missing & 0 & 0 & 0 & 0 \\
\hline
\end{tabular}


Table C-6. Descriptive statistics at the posttreatment examination for the SNB Angle (degrees).

\begin{tabular}{lccccc}
\hline \multirow{2}{*}{ Variable } & \multicolumn{2}{c}{ MARA } & & \multicolumn{2}{c}{ Edgewise } \\
\cline { 2 - 3 } \cline { 5 - 6 } & & & & & \\
& & & & & \\
Arithmales & Males & & & \\
Standard mean & 76.31 & 76.37 & & 76.10 & 75.77 \\
Standard error mean & 0.94 & 0.72 & & 0.64 & 0.56 \\
Upper 95\% CL & 78.27 & 77.84 & & 77.44 & 76.91 \\
Lower 95\% CL & 74.34 & 74.89 & & 74.76 & 74.63 \\
Sample size & 20 & 31 & & 20 & 31 \\
Variance & 17.55 & 16.21 & & 8.18 & 9.66 \\
Skewness & 1.20 & -0.04 & 0.12 & -0.55 \\
Kurtosis & 1.33 & -0.87 & -0.97 & 0.34 \\
Number missing & 0 & 0 & & 0 & 0 \\
\hline
\end{tabular}


Table C-7. Descriptive statistics at the posttreatment examination for the ANB Angle (degrees).

\begin{tabular}{lccccc}
\hline \multirow{2}{*}{ Variable } & \multicolumn{2}{c}{ MARA } & & \multicolumn{2}{c}{ Edgewise } \\
\cline { 2 - 3 } \cline { 5 - 6 } Females & Males & & Females & Males \\
Arithmetic mean & 3.29 & 3.25 & & 4.01 & 4.19 \\
Standard deviation & 1.70 & 1.71 & & 2.09 & 2.10 \\
Standard error mean & 0.38 & 0.31 & & 0.47 & 0.38 \\
Upper 95\% CL & 4.08 & 3.87 & & 4.98 & 4.96 \\
Lower 95\% CL & 2.49 & 2.62 & & 3.03 & 3.42 \\
Sample size & 20 & 31 & & 20 & 31 \\
Variance & 2.88 & 2.91 & & 4.37 & 4.40 \\
Skewness & 0.08 & -0.28 & 0.27 & 0.22 \\
Kurtosis & -0.57 & -0.82 & -1.09 & -0.70 \\
Number missing & 0 & 0 & 0 & 0 \\
\hline
\end{tabular}


Table C-8. Descriptive statistics at the posttreatment examination for the AOBO (mm).

\begin{tabular}{lccccc}
\hline \multirow{2}{*}{ Variable } & \multicolumn{2}{c}{ MARA } & & \multicolumn{2}{c}{ Edgewise } \\
\cline { 2 - 3 } \cline { 5 - 6 } & Females & Males & & Females & Males \\
Arithmetic mean & -0.08 & 0.97 & & 1.15 & 1.12 \\
Standard deviation & 1.88 & 2.06 & & 3.19 & 2.24 \\
Standard error mean & 0.42 & 0.37 & & 0.71 & 0.40 \\
Upper 95\% CL & 0.80 & 1.73 & & 2.64 & 1.94 \\
Lower 95\% CL & -0.96 & 0.22 & -0.35 & 0.30 \\
Sample size & 20 & 31 & 20 & 31 \\
Variance & 3.54 & 4.23 & 10.19 & 5.00 \\
Skewness & -0.63 & -0.02 & -0.41 & -0.11 \\
Kurtosis & -0.19 & -0.78 & 0.45 & -0.96 \\
Number missing & 0 & 0 & 0 & 0 \\
\hline
\end{tabular}


Table C-9. Descriptive statistics at the posttreatment examination for the FMA Angle (degrees).

\begin{tabular}{lccccc}
\hline \multirow{2}{*}{ Variable } & \multicolumn{2}{c}{ MARA } & & \multicolumn{2}{c}{ Edgewise } \\
\cline { 2 - 3 } \cline { 5 - 6 } & Females & Males & & Females & Males \\
\hline Arithmetic mean & 24.45 & 24.31 & & 25.02 & 25.92 \\
Standard deviation & 6.09 & 5.39 & & 6.17 & 4.94 \\
Standard error mean & 1.36 & 0.98 & & 1.38 & 0.89 \\
Upper 95\% CL & 27.30 & 26.32 & & 27.91 & 27.73 \\
Lower 95\% CL & 21.60 & 22.30 & & 22.13 & 24.11 \\
Sample size & 20 & 30 & 20 & 31 \\
Variance & 37.14 & 29.01 & 38.10 & 24.43 \\
Skewness & -0.28 & -0.09 & -0.58 & -0.66 \\
Kurtosis & -0.66 & -1.21 & 0.76 & 0.68 \\
Number missing & 0 & 1 & 0 & 0 \\
\hline
\end{tabular}


Table C-10. Descriptive statistics at the posttreatment examination for the IMPA (degrees).

\begin{tabular}{lccccc}
\hline \multirow{2}{*}{ Variable } & \multicolumn{2}{c}{ MARA } & & \multicolumn{2}{c}{ Edgewise } \\
\cline { 2 - 3 } \cline { 5 - 6 } & Females & Males & & Females & Males \\
\hline Arithmetic mean & 97.46 & 99.53 & & 97.88 & 97.87 \\
Standard deviation & 7.92 & 6.33 & & 7.26 & 7.17 \\
Standard error mean & 1.77 & 1.15 & & 1.62 & 1.29 \\
Upper 95\% CL & 101.17 & 101.89 & & 101.27 & 100.50 \\
Lower 95\% CL & 93.75 & 97.17 & 94.48 & 95.24 \\
Sample size & 20 & 30 & 20 & 31 \\
Variance & 62.68 & 40.02 & 52.77 & 51.43 \\
Skewness & -0.51 & -0.19 & 1.21 & -0.66 \\
Kurtosis & -0.56 & -0.42 & 1.43 & 0.11 \\
Number missing & 0 & 1 & 0 & 0 \\
\hline
\end{tabular}


Table C-11. Descriptive statistics at the posttreatment examination for the FMIA (degrees).

\begin{tabular}{lccccc}
\hline \multirow{2}{*}{ Variable } & \multicolumn{2}{c}{ MARA } & & \multicolumn{2}{c}{ Edgewise } \\
\cline { 2 - 3 } \cline { 5 - 6 } & Females & Males & & Females & Males \\
\hline Arithmetic mean & 58.10 & 56.50 & & 57.09 & 56.20 \\
Standard deviation & 6.29 & 6.85 & & 4.90 & 6.57 \\
Standard error mean & 1.41 & 1.23 & & 1.10 & 1.18 \\
Upper 95\% CL & 61.04 & 59.01 & & 59.38 & 58.61 \\
Lower 95\% CL & 55.16 & 53.99 & & 54.80 & 53.79 \\
Sample size & 20 & 31 & 20 & 31 \\
Variance & 39.52 & 46.95 & 24.00 & 43.15 \\
Skewness & 0.21 & 0.33 & 0.06 & 0.76 \\
Kurtosis & -0.16 & -0.50 & -0.14 & 0.11 \\
Number missing & 0 & 0 & 0 & 0 \\
\hline
\end{tabular}


Table C-12. Descriptive statistics at the posttreatment examination for the OP to FH Angle (degrees).

\begin{tabular}{lccccc}
\hline \multirow{2}{*}{ Variable } & \multicolumn{2}{c}{ MARA } & & \multicolumn{2}{c}{ Edgewise } \\
\cline { 2 - 3 } \cline { 5 - 6 } & & & & & \\
& & & & & \\
Arithmales & Males & & & \\
Standard mean & 8.43 & 8.27 & & 10.08 & 11.60 \\
Standard error mean & 0.98 & 0.84 & & 1.00 & 0.64 \\
Upper 95\% CL & 10.48 & 9.98 & & 12.18 & 12.91 \\
Lower 95\% CL & 6.37 & 6.55 & & 7.98 & 10.28 \\
Sample size & 20 & 31 & & 20 & 31 \\
Variance & 19.27 & 21.84 & 20.09 & 12.82 \\
Skewness & 0.15 & 0.16 & -0.58 & 0.07 \\
Kurtosis & -0.24 & -1.04 & -0.37 & 0.26 \\
Number missing & 0 & 0 & & 0 & 0 \\
\hline
\end{tabular}


Table C-13. Descriptive statistics at the posttreatment examination for the U1 to SeNa Angle (degrees).

\begin{tabular}{lccccc}
\hline \multirow{2}{*}{ Variable } & \multicolumn{2}{c}{ MARA } & & \multicolumn{2}{c}{ Edgewise } \\
\cline { 2 - 3 } \cline { 5 - 6 } & Females & Males & & Females & Males \\
Arithmetic mean & 103.50 & 105.24 & & 99.14 & 99.61 \\
Standard deviation & 7.48 & 6.56 & & 8.30 & 7.25 \\
Standard error mean & 1.67 & 1.18 & & 1.86 & 1.30 \\
Upper 95\% CL & 106.99 & 107.65 & & 103.02 & 102.27 \\
Lower 95\% CL & 100.00 & 102.83 & & 95.25 & 96.95 \\
Sample size & 20 & 31 & 20 & 31 \\
Variance & 55.89 & 43.08 & 68.91 & 52.61 \\
Skewness & -0.08 & -0.13 & 0.53 & 0.40 \\
Kurtosis & -0.87 & 0.70 & 0.66 & 0.03 \\
Number missing & 0 & 0 & 0 & 0 \\
\hline
\end{tabular}


Table C-14. Descriptive statistics at the posttreatment examination for the Interincisal Angle (degrees).

\begin{tabular}{lccccc}
\hline \multirow{2}{*}{ Variable } & \multicolumn{2}{c}{ MARA } & & \multicolumn{2}{c}{ Edgewise } \\
\cline { 2 - 3 } \cline { 5 - 6 } & Females & Males & & Females & Males \\
\hline Arithmetic mean & 124.28 & 122.32 & & 130.05 & 129.18 \\
Standard deviation & 7.77 & 7.26 & & 9.02 & 10.26 \\
Standard error mean & 1.74 & 1.30 & & 2.02 & 1.84 \\
Upper 95\% CL & 127.92 & 124.99 & & 134.27 & 132.95 \\
Lower 95\% CL & 120.64 & 119.66 & & 125.83 & 125.42 \\
Sample size & 20 & 31 & 20 & 31 \\
Variance & 60.34 & 52.73 & 81.38 & 105.35 \\
Skewness & -0.02 & -0.98 & -0.42 & -0.34 \\
Kurtosis & -0.58 & 1.69 & 0.25 & -0.89 \\
Number missing & 0 & 0 & 0 & 0 \\
\hline
\end{tabular}


Table C-15. Descriptive statistics at the posttreatment examination for the U1 to Na A Pt Angle (degrees).

\begin{tabular}{lccccc}
\hline \multirow{2}{*}{ Variable } & \multicolumn{2}{c}{ MARA } & & \multicolumn{2}{c}{ Edgewise } \\
\cline { 2 - 3 } \cline { 5 - 6 } & Females & Males & & Females & Males \\
\hline Arithmetic mean & 23.87 & 25.63 & & 19.03 & 19.66 \\
Standard deviation & 6.38 & 6.67 & & 8.47 & 7.99 \\
Standard error mean & 1.43 & 1.20 & & 1.89 & 1.43 \\
Upper 95\% CL & 26.86 & 28.08 & & 22.99 & 22.59 \\
Lower 95\% CL & 20.88 & 23.19 & & 15.07 & 16.73 \\
Sample size & 20 & 31 & 20 & 31 \\
Variance & 40.70 & 44.44 & 71.72 & 63.80 \\
Skewness & -0.49 & 0.40 & 0.47 & -0.21 \\
Kurtosis & -0.73 & 0.29 & 0.50 & -0.78 \\
Number missing & 0 & 0 & 0 & 0 \\
\hline
\end{tabular}


Table C-16. Descriptive statistics at the posttreatment examination for the U1 to $\mathrm{Na}$ A Pt (mm).

\begin{tabular}{lccccc}
\hline \multirow{2}{*}{ Variable } & \multicolumn{2}{c}{ MARA } & & \multicolumn{2}{c}{ Edgewise } \\
\cline { 2 - 3 } \cline { 5 - 6 } & Females & Males & & Females & Males \\
Arithmetic mean & 3.66 & 4.24 & & 1.42 & 1.47 \\
Standard deviation & 1.99 & 2.36 & & 2.79 & 3.00 \\
Standard error mean & 0.44 & 0.42 & & 0.62 & 0.54 \\
Upper 95\% CL & 4.58 & 5.11 & & 2.72 & 2.57 \\
Lower 95\% CL & 2.73 & 3.38 & & 0.11 & 0.37 \\
Sample size & 20 & 31 & 20 & 31 \\
Variance & 3.94 & 5.58 & & 7.81 & 9.00 \\
Skewness & -0.05 & 0.21 & -0.08 & -0.21 \\
Kurtosis & -0.70 & -0.72 & -0.63 & -0.73 \\
Number missing & 0 & 0 & 0 & 0 \\
\hline
\end{tabular}


Table C-17. Descriptive statistics at the posttreatment examination for the L1 to A Pg Angle (degrees).

\begin{tabular}{lccccc}
\hline \multirow{2}{*}{ Variable } & \multicolumn{2}{c}{ MARA } & & \multicolumn{2}{c}{ Edgewise } \\
\cline { 2 - 3 } \cline { 5 - 6 } & & & & & \\
& & & & & \\
Arithmales & Males & & & \\
Standard mean & 26.69 & 28.21 & & 25.27 & 25.55 \\
Standard error mean & 1.00 & 0.77 & & 1.16 & 0.99 \\
Upper 95\% CL & 28.78 & 29.78 & & 27.70 & 27.57 \\
Lower 95\% CL & 24.59 & 26.64 & & 22.84 & 23.53 \\
Sample size & 20 & 31 & & 20 & 31 \\
Variance & 20.08 & 18.28 & 26.98 & 30.30 \\
Skewness & 0.02 & 0.59 & 0.48 & -0.26 \\
Kurtosis & -0.09 & -0.72 & -0.29 & -0.10 \\
Number missing & 0 & 0 & 0 & 0 \\
\hline
\end{tabular}


Table C-18. Descriptive statistics at the posttreatment examination for the L1 to A Pg (mm).

\begin{tabular}{lccccc}
\hline \multirow{2}{*}{ Variable } & \multicolumn{2}{c}{ MARA } & & \multicolumn{2}{c}{ Edgewise } \\
\cline { 2 - 3 } \cline { 5 - 6 } & & & & & \\
& & & & & \\
Arithmemales & Males & & & Males \\
Standard deviation & 1.05 & 1.49 & & 2.37 & 1.96 \\
Standard error mean & 0.24 & 0.27 & & 0.53 & 0.35 \\
Upper 95\% CL & 3.01 & 2.97 & & 2.02 & 1.59 \\
Lower 95\% CL & 2.02 & 1.87 & & -0.21 & 0.16 \\
Sample size & 20 & 31 & 20 & 31 \\
Variance & 1.11 & 2.23 & & 5.63 & 3.83 \\
Skewness & -0.39 & -0.77 & -0.05 & 0.14 \\
Kurtosis & -0.10 & 0.31 & -0.57 & -1.15 \\
Number missing & 0 & 0 & 0 & 0 \\
\hline
\end{tabular}


Table C-19. Descriptive statistics at the posttreatment examination for the L1 to Na B Pt Angle (degrees).

\begin{tabular}{lccccc}
\hline \multirow{2}{*}{ Variable } & \multicolumn{2}{c}{ MARA } & & \multicolumn{2}{c}{ Edgewise } \\
\cline { 2 - 3 } \cline { 5 - 6 } & Females & Males & & Females & Males \\
\hline Arithmetic mean & 28.55 & 28.80 & & 26.76 & 26.98 \\
Standard deviation & 4.88 & 5.38 & & 6.09 & 6.32 \\
Standard error mean & 1.09 & 0.97 & & 1.36 & 1.14 \\
Upper 95\% CL & 30.84 & 30.77 & & 29.61 & 29.30 \\
Lower 95\% CL & 26.26 & 26.82 & & 23.91 & 24.66 \\
Sample size & 20 & 31 & 20 & 31 \\
Variance & 23.86 & 28.92 & 37.10 & 39.96 \\
Skewness & -0.45 & -0.11 & 0.57 & -0.60 \\
Kurtosis & -0.92 & -0.53 & 0.72 & 0.05 \\
Number missing & 0 & 0 & 0 & 0 \\
\hline
\end{tabular}


Table C-20. Descriptive statistics at the posttreatment examination for the L1 to $\mathrm{Na} B \mathrm{Pt}(\mathrm{mm})$.

\begin{tabular}{lccccc}
\hline \multirow{2}{*}{ Variable } & \multicolumn{2}{c}{ MARA } & & \multicolumn{2}{c}{ Edgewise } \\
\cline { 2 - 3 } \cline { 5 - 6 } & Females & Males & & Females & Males \\
Arithmetic mean & 5.16 & 5.37 & & 4.39 & 4.64 \\
Standard deviation & 1.28 & 2.16 & & 2.01 & 2.04 \\
Standard error mean & 0.29 & 0.39 & & 0.45 & 0.37 \\
Upper 95\% CL & 5.75 & 6.16 & & 5.33 & 5.39 \\
Lower 95\% CL & 4.56 & 4.58 & & 3.44 & 3.89 \\
Sample size & 20 & 31 & & 20 & 31 \\
Variance & 1.63 & 4.67 & & 4.05 & 4.18 \\
Skewness & 0.65 & -0.63 & 0.49 & -0.22 \\
Kurtosis & -0.39 & 0.60 & 0.87 & -0.87 \\
Number missing & 0 & 0 & 0 & 0 \\
\hline
\end{tabular}


Table C-21. Descriptive statistics at the posttreatment examination for the Facial Angle (degrees).

\begin{tabular}{lccccc}
\hline \multirow{2}{*}{ Variable } & \multicolumn{2}{c}{ MARA } & & \multicolumn{2}{c}{ Edgewise } \\
\cline { 2 - 3 } \cline { 5 - 6 } & Females & Males & & Females & Males \\
\hline Arithmetic mean & 87.51 & 86.68 & & 85.28 & 84.67 \\
Standard deviation & 3.97 & 3.67 & & 3.27 & 3.09 \\
Standard error mean & 0.89 & 0.66 & & 0.73 & 0.56 \\
Upper 95\% CL & 89.37 & 88.03 & & 86.81 & 85.81 \\
Lower 95\% CL & 85.65 & 85.34 & & 83.75 & 83.54 \\
Sample size & 20 & 31 & 20 & 31 \\
Variance & 15.78 & 13.47 & 10.68 & 9.56 \\
Skewness & 0.43 & 0.04 & 0.26 & -0.75 \\
Kurtosis & 0.34 & -0.89 & -0.77 & 0.51 \\
Number missing & 0 & 0 & 0 & 0 \\
\hline
\end{tabular}


Table C-22. Descriptive statistics at the posttreatment examination for the Mand Length (mm).

\begin{tabular}{lccccc}
\hline \multirow{2}{*}{ Variable } & \multicolumn{2}{c}{ MARA } & & \multicolumn{2}{c}{ Edgewise } \\
\cline { 2 - 3 } \cline { 5 - 6 } & & & & & \\
& & & & & \\
Arithmemales & Males & & & \\
Standard deviation & 6.37 & 6.47 & & 6.88 & 8.98 \\
Standard error mean & 1.42 & 1.18 & & 1.54 & 1.61 \\
Upper 95\% CL & 116.71 & 121.99 & 120.13 & 119.36 \\
Lower 95\% CL & 110.76 & 117.17 & 113.69 & 112.77 \\
Sample size & 20 & 30 & 20 & 31 \\
Variance & 40.54 & 41.80 & 47.32 & 80.60 \\
Skewness & -0.10 & -0.73 & 1.70 & -0.26 \\
Kurtosis & -0.38 & 1.45 & 4.27 & 0.35 \\
Number missing & 0 & 1 & 0 & 0 \\
\hline
\end{tabular}


Table C-23. Descriptive statistics at the posttreatment examination for the Sellato-A Point (mm).

\begin{tabular}{lccccc}
\hline \multirow{2}{*}{ Variable } & \multicolumn{2}{c}{ MARA } & & \multicolumn{2}{c}{ Edgewise } \\
\cline { 2 - 3 } \cline { 5 - 6 } & Females & Males & & Females & Males \\
\hline Arithmetic mean & 85.69 & 90.50 & & 87.18 & 86.45 \\
Standard deviation & 4.10 & 4.64 & 6.11 & 6.44 \\
Standard error mean & 0.92 & 0.83 & & 1.37 & 1.16 \\
Upper 95\% CL & 87.60 & 92.20 & & 90.04 & 88.81 \\
Lower 95\% CL & 83.77 & 88.80 & & 84.31 & 84.08 \\
Sample size & 20 & 31 & 20 & 31 \\
Variance & 16.82 & 21.49 & 37.35 & 41.51 \\
Skewness & -0.85 & -0.37 & -0.34 & 0.06 \\
Kurtosis & 1.24 & -0.50 & 2.60 & 0.86 \\
Number missing & 0 & 0 & 0 & 0 \\
\hline
\end{tabular}


Table C-24. Descriptive statistics at the posttreatment examination for the Sellato-B Point (mm).

\begin{tabular}{lccccc}
\hline \multirow{2}{*}{ Variable } & \multicolumn{2}{c}{ MARA } & & \multicolumn{2}{c}{ Edgewise } \\
\cline { 2 - 3 } \cline { 5 - 6 } & Females & Males & & Females & Males \\
\hline Arithmetic mean & 108.71 & 113.80 & & 108.95 & 108.22 \\
Standard deviation & 5.91 & 5.53 & 8.19 & 8.50 \\
Standard error mean & 1.32 & 0.99 & & 1.83 & 1.53 \\
Upper 95\% CL & 111.48 & 115.83 & & 112.78 & 111.34 \\
Lower 95\% CL & 105.94 & 111.77 & 105.12 & 105.10 \\
Sample size & 20 & 31 & 20 & 31 \\
Variance & 34.96 & 30.61 & 67.05 & 72.23 \\
Skewness & -0.33 & 0.43 & -0.20 & 0.33 \\
Kurtosis & -0.02 & -0.11 & 1.60 & 1.94 \\
Number missing & 0 & 0 & 0 & 0 \\
\hline
\end{tabular}


Table C-25. Descriptive statistics at the posttreatment examination for the Overbite (mm).

\begin{tabular}{lccccc}
\hline \multirow{2}{*}{ Variable } & \multicolumn{2}{c}{ MARA } & & \multicolumn{2}{c}{ Edgewise } \\
\cline { 2 - 3 } \cline { 5 - 6 } & & & & & \\
Arithmetes & Males & & & \\
Standard deviation & 0.88 & 0.89 & & 1.01 & 0.92 \\
Standard error mean & 0.20 & 0.16 & & 0.23 & 0.16 \\
Upper 95\% CL & 2.26 & 2.16 & & 1.36 & 1.43 \\
Lower 95\% CL & 1.43 & 1.51 & & 0.42 & 0.76 \\
Sample size & 20 & 31 & & 20 & 31 \\
Variance & 0.78 & 0.79 & & 1.02 & 0.84 \\
Skewness & -0.23 & 0.47 & 0.46 & 1.18 \\
Kurtosis & -0.51 & -1.12 & -0.65 & 1.53 \\
Number missing & 0 & 0 & 0 & 0 \\
\hline
\end{tabular}


Table C-26. Descriptive statistics at the posttreatment examination for the Overjet (mm).

\begin{tabular}{lccccc}
\hline \multirow{2}{*}{ Variable } & \multicolumn{2}{c}{ MARA } & & \multicolumn{2}{c}{ Edgewise } \\
\cline { 2 - 3 } \cline { 5 - 6 } & Females & Males & & Females & Males \\
Arithmetic mean & 3.42 & 3.59 & & 2.75 & 3.18 \\
Standard deviation & 0.70 & 0.74 & & 1.06 & 1.21 \\
Standard error mean & 0.16 & 0.13 & & 0.24 & 0.22 \\
Upper 95\% CL & 3.75 & 3.86 & & 3.25 & 3.63 \\
Lower 95\% CL & 3.09 & 3.32 & & 2.25 & 2.74 \\
Sample size & 20 & 31 & 20 & 31 \\
Variance & 0.49 & 0.54 & & 1.13 & 1.46 \\
Skewness & 0.49 & 0.84 & 0.25 & 0.61 \\
Kurtosis & 0.49 & 0.95 & -0.03 & 0.04 \\
Number missing & 0 & 0 & 0 & 0 \\
\hline
\end{tabular}


Table C-27. Descriptive statistics at the posttreatment examination for the A Pt to Na-Perp (mm).

\begin{tabular}{lccccc}
\hline \multirow{2}{*}{ Variable } & \multicolumn{2}{c}{ MARA } & & \multicolumn{2}{c}{ Edgewise } \\
\cline { 2 - 3 } \cline { 5 - 6 } & Females & Males & & Females & Males \\
\hline Arithmetic mean & -0.04 & -1.70 & & -2.12 & -2.71 \\
Standard deviation & 3.59 & 4.17 & & 3.94 & 4.48 \\
Standard error mean & 0.80 & 0.75 & & 0.88 & 0.80 \\
Upper 95\% CL & 1.64 & -0.18 & & -0.27 & -1.06 \\
Lower 95\% CL & -1.72 & -3.23 & -3.96 & -4.35 \\
Sample size & 20 & 31 & 20 & 31 \\
Variance & 12.91 & 17.36 & 15.54 & 20.03 \\
Skewness & 0.66 & 0.19 & 0.85 & -0.53 \\
Kurtosis & -0.33 & -0.35 & 0.76 & -0.19 \\
Number missing & 0 & 0 & 0 & 0 \\
\hline
\end{tabular}


Table C-28. Descriptive statistics at the posttreatment examination for the B Pt to Na-Perp (mm).

\begin{tabular}{lccccc}
\hline \multirow{2}{*}{ Variable } & \multicolumn{2}{c}{ MARA } & & \multicolumn{2}{c}{ Edgewise } \\
\cline { 2 - 3 } \cline { 5 - 6 } & Females & Males & & Females & Males \\
\hline Arithmetic mean & -5.37 & -8.66 & & -10.25 & -11.84 \\
Standard deviation & 6.88 & 6.86 & & 5.46 & 5.82 \\
Standard error mean & 1.54 & 1.23 & & 1.22 & 1.05 \\
Upper 95\% CL & -2.15 & -6.15 & & -7.69 & -9.70 \\
Lower 95\% CL & -8.58 & -11.18 & -12.80 & -13.97 \\
Sample size & 20 & 31 & & 20 & 31 \\
Variance & 47.28 & 47.05 & 29.85 & 33.91 \\
Skewness & 0.48 & -0.11 & 0.21 & -0.71 \\
Kurtosis & -0.33 & -0.54 & 0.00 & -0.14 \\
Number missing & 0 & 0 & 0 & 0 \\
\hline
\end{tabular}




\section{APPENDIX D.}

DESCRIPTIVE STATISTICS, BY TREATMENT AND SEX, FOR THE INTREATMENT CHANGE IN CEPHALOMETRIC VARIABLES. 
Table D-1. Descriptive statistics at the in-treatment changes for the $\mathrm{Z}$ angle (degrees).

\begin{tabular}{lccccc}
\hline \multirow{2}{*}{ Variable } & \multicolumn{2}{c}{ MARA } & & \multicolumn{2}{c}{ Edgewise } \\
\cline { 2 - 3 } \cline { 5 - 6 } & Females & Males & & Females & Males \\
\hline Arithmetic mean & 4.58 & 2.09 & & 6.38 & 8.32 \\
Standard deviation & 6.52 & 8.64 & & 7.71 & 9.24 \\
Standard error mean & 1.54 & 1.80 & & 1.77 & 1.69 \\
Upper 95\% CL & 7.82 & 5.82 & & 10.09 & 11.77 \\
Lower 95\% CL & 1.34 & -1.65 & & 2.66 & 4.86 \\
Sample size & 18 & 23 & & 19 & 30 \\
Variance & 42.45 & 74.60 & & 59.38 & 85.45 \\
Skewness & 0.20 & -1.20 & -0.27 & 0.24 \\
Kurtosis & 1.29 & 1.94 & -0.55 & 2.96 \\
Number missing & 2 & 8 & 1 & 1 \\
T-test (one-sample) & 2.98 & 1.16 & 3.61 & 4.93 \\
Probability value & 0.0083 & 0.2590 & 0.0020 & $<0.0001$ \\
\hline
\end{tabular}


Table D-2. Descriptive statistics at the in-treatment changes for the E Plane (mm).

\begin{tabular}{lccccc}
\hline \multirow{2}{*}{ Variable } & \multicolumn{2}{c}{ MARA } & & \multicolumn{2}{c}{ Edgewise } \\
\cline { 2 - 3 } \cline { 5 - 6 } & Females & Males & & Females & Males \\
Arithmetic mean & -1.64 & -1.34 & & -2.22 & -3.04 \\
Standard deviation & 1.68 & 2.95 & & 2.09 & 2.21 \\
Standard error mean & 0.40 & 0.68 & & 0.48 & 0.40 \\
Upper 95\% CL & -0.81 & 0.08 & & -1.21 & -2.22 \\
Lower 95\% CL & -2.48 & -2.76 & -3.22 & -3.87 \\
Sample size & 18 & 19 & & 19 & 30 \\
Variance & 2.82 & 8.70 & & 4.37 & 4.88 \\
Skewness & 0.29 & 0.73 & 0.24 & 0.44 \\
Kurtosis & -0.15 & 1.11 & -0.08 & 0.37 \\
Number missing & 2 & 12 & 1 & 1 \\
T-test (one-sample) & -4.16 & -1.98 & -4.62 & -7.55 \\
Probability value & 0.0007 & 0.0628 & 0.0002 & $<0.0001$ \\
\hline
\end{tabular}


Table D-3. Descriptive statistics at the in-treatment changes for the Y-Axis Angle (degrees).

\begin{tabular}{lccccc}
\hline \multirow{2}{*}{ Variable } & \multicolumn{2}{c}{ MARA } & & \multicolumn{2}{c}{ Edgewise } \\
\cline { 2 - 3 } \cline { 5 - 6 } Females & Males & & Females & Males \\
Arithmetic mean & 0.39 & 0.30 & & 0.13 & 0.37 \\
Standard deviation & 1.99 & 1.46 & & 1.46 & 2.32 \\
Standard error mean & 0.45 & 0.27 & & 0.33 & 0.42 \\
Upper 95\% CL & 1.32 & 0.84 & & 0.81 & 1.22 \\
Lower 95\% CL & -0.55 & -0.24 & -0.56 & -0.48 \\
Sample size & 20 & 30 & 20 & 31 \\
Variance & 3.97 & 2.13 & & 2.13 & 5.39 \\
Skewness & -0.19 & 0.53 & -0.59 & -0.83 \\
Kurtosis & 0.51 & 0.06 & -0.18 & 4.08 \\
Number missing & 0 & 1 & 0 & 0 \\
T-test (one-sample) & 0.86 & 1.13 & 0.38 & 0.89 \\
Probability value & 0.3983 & 0.2693 & 0.7058 & 0.3805 \\
\hline
\end{tabular}


Table D-4. Descriptive statistics at the in-treatment changes for the NAP Angle (degrees).

\begin{tabular}{lccccc}
\hline \multirow{2}{*}{ Variable } & \multicolumn{2}{c}{ MARA } & & \multicolumn{2}{c}{ Edgewise } \\
\cline { 2 - 3 } \cline { 5 - 6 } & & & & & \\
Arithmetic mean & -5.02 & -6.46 & & -6.17 & -4.35 \\
Standard deviation & 3.71 & 3.71 & & 3.72 & 3.18 \\
Standard error mean & 0.83 & 0.67 & & 0.83 & 0.57 \\
Upper 95\% CL & -3.28 & -5.10 & & -4.42 & -3.18 \\
Lower 95\% CL & -6.75 & -7.82 & -7.91 & -5.52 \\
Sample size & 20 & 31 & & 20 & 31 \\
Variance & 13.74 & 13.80 & & 13.85 & 10.12 \\
Skewness & -0.37 & -0.23 & -0.33 & -1.01 \\
Kurtosis & -0.47 & -0.49 & -0.62 & 1.60 \\
Number missing & 0 & 0 & 0 & 0 \\
T-test (one-sample) & -6.05 & -9.68 & -7.41 & -7.62 \\
Probability value & $<0.0001$ & $<0.0001$ & $<0.0001$ & $<0.0001$ \\
\hline
\end{tabular}


Table D-5. Descriptive statistics at the in-treatment changes for the SNA Angle (degrees).

\begin{tabular}{lccccc}
\hline \multirow{2}{*}{ Variable } & \multicolumn{2}{c}{ MARA } & & \multicolumn{2}{c}{ Edgewise } \\
\cline { 2 - 3 } \cline { 5 - 6 } & Females & Males & & Females & Males \\
Arithmetic mean & -1.53 & -1.71 & & -2.29 & -1.28 \\
Standard deviation & 2.40 & 1.83 & & 1.24 & 1.78 \\
Standard error mean & 0.54 & 0.33 & & 0.28 & 0.32 \\
Upper 95\% CL & -0.40 & -1.04 & & -1.71 & -0.63 \\
Lower 95\% CL & -2.65 & -2.38 & -2.86 & -1.94 \\
Sample size & 20 & 31 & 20 & 31 \\
Variance & 5.76 & 3.34 & & 1.53 & 3.17 \\
Skewness & 0.40 & 0.59 & 0.11 & 0.61 \\
Kurtosis & 0.00 & -0.49 & -0.65 & 1.24 \\
Number missing & 0 & 0 & 0 & 0 \\
T-test (one-sample) & -2.84 & -5.21 & -8.26 & -4.02 \\
Probability value & 0.0104 & $<0.0001$ & $<0.0001$ & 0.0004 \\
\hline
\end{tabular}


Table D-6. Descriptive statistics at the in-treatment changes for the SNB Angle (degrees).

\begin{tabular}{lccccc}
\hline \multirow{2}{*}{ Variable } & \multicolumn{2}{c}{ MARA } & & \multicolumn{2}{c}{ Edgewise } \\
\cline { 2 - 3 } \cline { 5 - 6 } & Females & Males & & Females & Males \\
Arithmetic mean & 0.53 & 0.76 & & -0.15 & -0.02 \\
Standard deviation & 2.12 & 1.62 & & 1.19 & 1.95 \\
Standard error mean & 0.47 & 0.29 & & 0.27 & 0.35 \\
Upper 95\% CL & 1.52 & 1.35 & & 0.41 & 0.70 \\
Lower 95\% CL & -0.47 & 0.16 & & -0.70 & -0.73 \\
Sample size & 20 & 31 & 20 & 31 \\
Variance & 4.48 & 2.62 & & 1.42 & 3.80 \\
Skewness & -0.24 & 0.04 & -0.38 & 1.63 \\
Kurtosis & -0.26 & 0.27 & -1.06 & 5.79 \\
Number missing & 0 & 0 & 0 & 0 \\
T-test (one-sample) & 1.11 & 2.61 & -0.54 & -0.05 \\
Probability value & 0.2812 & 0.0140 & 0.5931 & 0.9636 \\
\hline
\end{tabular}


Table D-7. Descriptive statistics at the in-treatment changes for the ANB Angle (degrees).

\begin{tabular}{lccccc}
\hline \multirow{2}{*}{ Variable } & \multicolumn{2}{c}{ MARA } & & \multicolumn{2}{c}{ Edgewise } \\
\cline { 2 - 3 } \cline { 5 - 6 } & Females & Males & & Females & Males \\
Arithmetic mean & -2.04 & -2.48 & & -2.14 & -1.26 \\
Standard deviation & 1.63 & 1.36 & & 1.43 & 1.42 \\
Standard error mean & 0.36 & 0.24 & & 0.32 & 0.26 \\
Upper 95\% CL & -1.28 & -1.98 & -1.46 & -0.74 \\
Lower 95\% CL & -2.80 & -2.98 & -2.81 & -1.79 \\
Sample size & 20 & 31 & 20 & 31 \\
Variance & 2.65 & 1.86 & 2.06 & 2.02 \\
Skewness & -0.39 & 0.07 & -0.15 & -0.49 \\
Kurtosis & -0.66 & -1.01 & -1.38 & 0.29 \\
Number missing & 0 & 0 & 0 & 0 \\
T-test (one-sample) & -5.60 & -10.12 & -6.66 & -4.95 \\
Probability value & $<0.0001$ & $<0.0001$ & $<0.0001$ & $<0.0001$ \\
\hline
\end{tabular}


Table D-8. Descriptive statistics at the in-treatment changes for the AOBO (mm).

\begin{tabular}{lccccc}
\hline \multirow{2}{*}{ Variable } & \multicolumn{2}{c}{ MARA } & & \multicolumn{2}{c}{ Edgewise } \\
\cline { 2 - 3 } \cline { 5 - 6 } & Females & Males & & Females & Males \\
\hline Arithmetic mean & -2.84 & -2.55 & & -2.42 & -2.24 \\
Standard deviation & 2.55 & 2.29 & & 2.33 & 2.88 \\
Standard error mean & 0.57 & 0.41 & & 0.52 & 0.52 \\
Upper 95\% CL & -1.65 & -1.70 & & -1.32 & -1.18 \\
Lower 95\% CL & -4.03 & -3.39 & & -3.51 & -3.29 \\
Sample size & 20 & 31 & & 20 & 31 \\
Variance & 6.50 & 5.25 & & 5.44 & 8.29 \\
Skewness & -0.23 & -0.11 & -0.17 & -0.31 \\
Kurtosis & 1.07 & -0.96 & -0.84 & 0.67 \\
Number missing & 0 & 0 & 0 & 0 \\
T-test (one-sample) & -4.98 & -6.18 & -4.63 & -4.33 \\
Probability value & $<0.0001$ & $<0.0001$ & 0.0002 & 0.0002 \\
\hline
\end{tabular}


Table D-9. Descriptive statistics at the in-treatment changes for the FMA Angle (degrees).

\begin{tabular}{lccccc}
\hline \multirow{2}{*}{ Variable } & \multicolumn{2}{c}{ MARA } & & \multicolumn{2}{c}{ Edgewise } \\
\cline { 2 - 3 } \cline { 5 - 6 } Females & Males & & Females & Males \\
Arithmetic mean & -0.88 & -0.25 & & -1.88 & -0.02 \\
Standard deviation & 3.33 & 2.82 & & 2.46 & 2.21 \\
Standard error mean & 0.74 & 0.52 & & 0.55 & 0.40 \\
Upper 95\% CL & 0.68 & 0.81 & & -0.72 & 0.79 \\
Lower 95\% CL & -2.44 & -1.30 & -3.03 & -0.83 \\
Sample size & 20 & 30 & 20 & 31 \\
Variance & 11.09 & 7.97 & & 6.06 & 4.87 \\
Skewness & -0.44 & -0.82 & -0.08 & 0.55 \\
Kurtosis & 0.74 & 0.51 & 0.06 & 0.91 \\
Number missing & 0 & 1 & 0 & 0 \\
T-test (one-sample) & -1.18 & -0.48 & -3.41 & -0.06 \\
Probability value & 0.2518 & 0.6359 & 0.0030 & 0.9550 \\
\hline
\end{tabular}


Table D-10. Descriptive statistics at the in-treatment changes for the IMPA (degrees).

\begin{tabular}{lccccc}
\hline \multirow{2}{*}{ Variable } & \multicolumn{2}{c}{ MARA } & & \multicolumn{2}{c}{ Edgewise } \\
\cline { 2 - 3 } \cline { 5 - 6 } Females & Males & & Females & Males \\
Arithmetic mean & 4.03 & 1.77 & & 2.73 & 2.52 \\
Standard deviation & 5.59 & 7.45 & & 8.16 & 6.85 \\
Standard error mean & 1.25 & 1.36 & & 1.82 & 1.23 \\
Upper 95\% CL & 6.65 & 4.55 & & 6.54 & 5.04 \\
Lower 95\% CL & 1.41 & -1.02 & & -1.09 & 0.01 \\
Sample size & 20 & 30 & & 20 & 31 \\
Variance & 31.22 & 55.57 & 66.60 & 46.99 \\
Skewness & 0.25 & 0.47 & 0.17 & -0.08 \\
Kurtosis & -0.85 & -0.05 & -0.21 & -0.82 \\
Number missing & 0 & 1 & 0 & 0 \\
T-test (one-sample) & 3.23 & 1.30 & 1.49 & 2.05 \\
Probability value & 0.0045 & 0.2045 & 0.1518 & 0.0493 \\
\hline
\end{tabular}


Table D-11. Descriptive statistics at the in-treatment changes for the FMIA (degrees).

\begin{tabular}{lccccc}
\hline \multirow{2}{*}{ Variable } & \multicolumn{2}{c}{ MARA } & & \multicolumn{2}{c}{ Edgewise } \\
\cline { 2 - 3 } \cline { 5 - 6 } & & & & & \\
Females & Males & & & \\
Arithmetic mean & -3.16 & -1.41 & & -0.86 & -2.61 \\
Standard deviation & 5.34 & 7.31 & & 7.54 & 6.57 \\
Standard error mean & 1.19 & 1.31 & & 1.69 & 1.18 \\
Upper 95\% CL & -0.66 & 1.27 & & 2.67 & -0.20 \\
Lower 95\% CL & -5.65 & -4.09 & & -4.38 & -5.02 \\
Sample size & 31 & 31 & & 20 & 31 \\
Variance & 28.50 & 53.46 & & 56.84 & 43.20 \\
Skewness & -0.43 & 0.07 & -0.07 & 0.09 \\
Kurtosis & -0.44 & -0.44 & -0.02 & -0.90 \\
Number missing & 0 & 0 & 0 & 0 \\
T-test (one-sample) & -2.64 & -1.08 & -0.51 & -2.21 \\
Probability value & 0.0160 & 0.2905 & 0.6179 & 0.0350 \\
\hline
\end{tabular}


Table D-12. Descriptive statistics at the in-treatment changes for the OP to FH Angle (degrees).

\begin{tabular}{lccccc}
\hline \multirow{2}{*}{ Variable } & \multicolumn{2}{c}{ MARA } & & \multicolumn{2}{c}{ Edgewise } \\
\cline { 2 - 3 } \cline { 5 - 6 } Females & Males & & Females & Males \\
Arithmetic mean & -0.33 & -0.47 & & 0.08 & 2.15 \\
Standard deviation & 3.53 & 2.78 & & 2.73 & 2.90 \\
Standard error mean & 0.79 & 0.50 & & 0.61 & 0.52 \\
Upper 95\% CL & 1.32 & 0.55 & & 1.36 & 3.22 \\
Lower 95\% CL & -1.98 & -1.49 & -1.20 & 1.09 \\
Sample size & 20 & 31 & 20 & 31 \\
Variance & 12.49 & 7.73 & & 7.46 & 8.38 \\
Skewness & 0.11 & -0.06 & -0.25 & 0.93 \\
Kurtosis & 0.48 & -0.69 & -0.38 & 0.34 \\
Number missing & 0 & 0 & 0 & 0 \\
T-test (one-sample) & -0.42 & -0.95 & 0.13 & 4.14 \\
Probability value & 0.6809 & 0.3499 & 0.8972 & 0.0003 \\
\hline
\end{tabular}


Table D-13. Descriptive statistics at the in-treatment changes for the U1 to SeNa Angle (degrees).

\begin{tabular}{lccccc}
\hline \multirow{2}{*}{ Variable } & \multicolumn{2}{c}{ MARA } & & \multicolumn{2}{c}{ Edgewise } \\
\cline { 2 - 3 } \cline { 5 - 6 } & Females & Males & & Females & Males \\
\hline Arithmetic mean & 1.62 & 2.80 & & -4.12 & -9.30 \\
Standard deviation & 9.14 & 9.13 & & 12.10 & 18.86 \\
Standard error mean & 2.04 & 1.64 & & 2.71 & 3.39 \\
Upper 95\% CL & 5.90 & 6.15 & & 1.54 & -2.38 \\
Lower 95\% CL & -2.66 & -0.55 & & -9.78 & -16.22 \\
Sample size & 20 & 31 & & 20 & 31 \\
Variance & 83.58 & 83.44 & & 146.37 & 355.77 \\
Skewness & -0.27 & 0.06 & 0.36 & -2.89 \\
Kurtosis & 0.69 & 0.33 & -1.38 & 11.84 \\
Number missing & 0 & 0 & 0 & 0 \\
T-test (one-sample) & 0.79 & 1.70 & -1.52 & -2.75 \\
Probability value & 0.4379 & 0.0986 & 0.1442 & 0.0101 \\
\hline
\end{tabular}


Table D-14. Descriptive statistics at the in-treatment changes for the Interincisal Angle (degrees).

\begin{tabular}{lccccc}
\hline \multirow{2}{*}{ Variable } & \multicolumn{2}{c}{ MARA } & & \multicolumn{2}{c}{ Edgewise } \\
\cline { 2 - 3 } \cline { 5 - 6 } & Females & Males & & Females & Males \\
\hline Arithmetic mean & -5.43 & -3.86 & & 2.74 & 4.00 \\
Standard deviation & 11.53 & 14.48 & & 17.11 & 12.24 \\
Standard error mean & 2.58 & 2.60 & & 3.83 & 2.20 \\
Upper 95\% CL & -0.03 & 1.45 & & 10.74 & 8.49 \\
Lower 95\% CL & -10.82 & -9.17 & & -5.27 & -0.49 \\
Sample size & 20 & 31 & 20 & 31 \\
Variance & 132.94 & 209.58 & 292.70 & 149.77 \\
Skewness & 0.03 & -0.23 & -0.24 & -0.17 \\
Kurtosis & 0.37 & 0.23 & -0.92 & -1.13 \\
Number missing & 0 & 0 & 0 & 0 \\
T-test (one-sample) & -2.10 & -1.49 & 0.71 & 1.82 \\
Probability value & 0.0489 & 0.1476 & 0.4833 & 0.0790 \\
\hline
\end{tabular}


Table D-15. Descriptive statistics at the in-treatment changes for the U1 to Na A Pt Angle (degrees).

\begin{tabular}{lccccc}
\hline \multirow{2}{*}{ Variable } & \multicolumn{2}{c}{ MARA } & & \multicolumn{2}{c}{ Edgewise } \\
\cline { 2 - 3 } \cline { 5 - 6 } & Females & Males & & Females & Males \\
Arithmetic mean & 3.13 & 4.51 & & -1.85 & -4.63 \\
Standard deviation & 9.41 & 9.65 & & 12.33 & 11.74 \\
Standard error mean & 2.10 & 1.73 & & 2.76 & 2.11 \\
Upper 95\% CL & 7.53 & 8.05 & & 3.92 & -0.32 \\
Lower 95\% CL & -1.28 & 0.97 & -7.62 & -8.93 \\
Sample size & 20 & 31 & 20 & 31 \\
Variance & 88.54 & 93.05 & & 152.04 & 137.77 \\
Skewness & -0.27 & 0.12 & 0.33 & -0.39 \\
Kurtosis & 0.99 & 0.27 & -1.25 & -0.53 \\
Number missing & 0 & 0 & 0 & 0 \\
T-test (one-sample) & 1.49 & 2.60 & -0.67 & -2.19 \\
Probability value & 0.1539 & 0.0142 & 0.5103 & 0.0361 \\
\hline
\end{tabular}


Table D-16. Descriptive statistics at the in-treatment changes for the U1 to Na A Pt (mm).

\begin{tabular}{lccccc}
\hline \multirow{2}{*}{ Variable } & \multicolumn{2}{c}{ MARA } & & \multicolumn{2}{c}{ Edgewise } \\
\cline { 2 - 3 } \cline { 5 - 6 } & Females & Males & & Females & Males \\
Arithmetic mean & 1.16 & 1.62 & & -1.26 & -2.90 \\
Standard deviation & 3.30 & 2.96 & & 3.66 & 4.01 \\
Standard error mean & 0.74 & 0.53 & & 0.82 & 0.72 \\
Upper 95\% CL & 2.71 & 2.70 & & 0.46 & -1.43 \\
Lower 95\% CL & -0.39 & 0.54 & -2.98 & -4.37 \\
Sample size & 20 & 31 & & 20 & 31 \\
Variance & 10.91 & 8.74 & & 13.43 & 16.06 \\
Skewness & 1.03 & -0.18 & 0.17 & 0.15 \\
Kurtosis & 0.39 & 0.45 & -0.65 & -0.72 \\
Number missing & 0 & 0 & 0 & 0 \\
T-test (one-sample) & 1.57 & 3.05 & -1.54 & -4.03 \\
Probability value & 0.1328 & 0.0047 & 0.1406 & 0.0004 \\
\hline
\end{tabular}


Table D-17. Descriptive statistics at the in-treatment changes for the L1 to A Pg Angle (degrees).

\begin{tabular}{lccccc}
\hline \multirow{2}{*}{ Variable } & \multicolumn{2}{c}{ MARA } & & \multicolumn{2}{c}{ Edgewise } \\
\cline { 2 - 3 } \cline { 5 - 6 } & & & & & \\
& & & & & \\
Arithmetes & & & & & \\
Sales & & & & \\
Standard deviation & 5.51 & 7.65 & & 7.84 & 6.89 \\
Standard error mean & 1.23 & 1.37 & & 1.75 & 1.23 \\
Upper 95\% CL & 10.09 & 8.60 & & 8.66 & 7.39 \\
Lower 95\% CL & 4.93 & 2.99 & & 1.32 & 2.38 \\
Sample size & 20 & 31 & & 20 & 31 \\
Variance & 30.32 & 58.50 & 61.43 & 46.59 \\
Skewness & 0.05 & 0.78 & -0.20 & 0.04 \\
Kurtosis & -0.49 & 0.91 & -0.34 & -1.05 \\
Number missing & 0 & 0 & 0 & 0 \\
T-test (one-sample) & 6.10 & 4.22 & 2.85 & 3.99 \\
Probability value & $<0.0001$ & 0.0002 & 0.0103 & 0.0004 \\
\hline
\end{tabular}


Table D-18. Descriptive statistics at the in-treatment changes for the L1 to A Pg (mm).

\begin{tabular}{lccccc}
\hline \multirow{2}{*}{ Variable } & \multicolumn{2}{c}{ MARA } & & \multicolumn{2}{c}{ Edgewise } \\
\cline { 2 - 3 } \cline { 5 - 6 } & & & & & \\
& & & & & \\
Arithmetes & & & & \\
Sales & & & & \\
Standard deviation & 1.75 & 2.42 & & 2.30 & 2.28 \\
Standard error mean & 0.39 & 0.44 & & 0.52 & 0.41 \\
Upper 95\% CL & 3.37 & 2.81 & & 1.23 & 1.25 \\
Lower 95\% CL & 1.74 & 1.03 & & -0.92 & -0.43 \\
Sample size & 20 & 31 & & 20 & 31 \\
Variance & 3.06 & 5.88 & & 5.31 & 5.21 \\
Skewness & -0.15 & -0.77 & -0.34 & -0.05 \\
Kurtosis & 0.56 & 0.46 & -0.45 & -0.22 \\
Number missing & 0 & 0 & 0 & 0 \\
T-test (one-sample) & 6.53 & 4.41 & 0.30 & 1.00 \\
Probability value & $<0.0001$ & 0.0001 & 0.7669 & 0.3256 \\
\hline
\end{tabular}


Table D-19. Descriptive statistics at the in-treatment changes for the L1 to Na B Pt Angle (degrees).

\begin{tabular}{lccccc}
\hline \multirow{2}{*}{ Variable } & \multicolumn{2}{c}{ MARA } & & \multicolumn{2}{c}{ Edgewise } \\
\cline { 2 - 3 } \cline { 5 - 6 } & Females & Males & & Females & Males \\
Arithmetic mean & 4.33 & 1.85 & & 1.08 & 1.91 \\
Standard deviation & 5.27 & 7.09 & & 7.65 & 6.33 \\
Standard error mean & 1.18 & 1.27 & & 1.71 & 1.14 \\
Upper 95\% CL & 6.79 & 4.45 & & 4.66 & 4.23 \\
Lower 95\% CL & 1.86 & -0.75 & & -2.50 & -0.41 \\
Sample size & 20 & 31 & & 20 & 31 \\
Variance & 27.79 & 50.27 & & 58.50 & 40.02 \\
Skewness & -0.16 & 0.11 & 0.10 & -0.02 \\
Kurtosis & 0.11 & -0.78 & 0.03 & -0.74 \\
Number missing & 0 & 0 & 0 & 0 \\
T-test (one-sample) & 3.67 & 1.45 & 0.63 & 1.68 \\
Probability value & 0.0016 & 0.1570 & 0.5352 & 0.1037 \\
\hline
\end{tabular}


Table D-20. Descriptive statistics at the in-treatment changes for the L1 to Na B $\mathrm{Pt}(\mathrm{mm})$.

\begin{tabular}{lccccc}
\hline \multirow{2}{*}{ Variable } & \multicolumn{2}{c}{ MARA } & & \multicolumn{2}{c}{ Edgewise } \\
\cline { 2 - 3 } \cline { 5 - 6 } & & & & & \\
& & & & & \\
Arithmales & Males & & & \\
Standard mean & 1.56 & 0.63 & & -0.46 & 0.14 \\
Standard error mean & 0.35 & 0.64 & & 0.54 & 0.37 \\
Upper 95\% CL & 2.29 & 1.93 & & 0.67 & 0.88 \\
Lower 95\% CL & 0.83 & -0.68 & & -1.59 & -0.61 \\
Sample size & 20 & 31 & & 20 & 31 \\
Variance & 2.43 & 12.65 & & 5.80 & 4.16 \\
Skewness & -0.09 & -0.25 & -0.71 & -1.07 \\
Kurtosis & -0.93 & 0.58 & -0.06 & 2.79 \\
Number missing & 0 & 0 & 0 & 0 \\
T-test (one-sample) & 4.47 & 0.98 & -0.85 & 0.37 \\
Probability value & 0.0003 & 0.3326 & 0.4035 & 0.7142 \\
\hline
\end{tabular}


Table D-21. Descriptive statistics at the in-treatment changes for the Facial Angle (degrees).

\begin{tabular}{lccccc}
\hline \multirow{2}{*}{ Variable } & \multicolumn{2}{c}{ MARA } & & \multicolumn{2}{c}{ Edgewise } \\
\cline { 2 - 3 } \cline { 5 - 6 } Females & Males & & Females & Males \\
Arithmetic mean & 1.53 & 0.94 & & 1.07 & -0.01 \\
Standard deviation & 2.64 & 2.43 & & 1.88 & 2.34 \\
Standard error mean & 0.59 & 0.44 & & 0.42 & 0.42 \\
Upper 95\% CL & 2.77 & 1.83 & & 1.95 & 0.85 \\
Lower 95\% CL & 0.29 & 0.05 & 0.19 & -0.86 \\
Sample size & 20 & 31 & 20 & 31 \\
Variance & 6.98 & 5.91 & 3.52 & 5.46 \\
Skewness & 0.25 & 0.23 & -0.20 & -0.19 \\
Kurtosis & 0.26 & -0.98 & -1.13 & 0.41 \\
Number missing & 0 & 0 & 0 & 0 \\
T-test (one-sample) & 2.59 & 2.15 & 2.55 & -0.02 \\
Probability value & 0.0179 & 0.0398 & 0.0195 & 0.9878 \\
\hline
\end{tabular}


Table D-22. Descriptive statistics at the in-treatment changes for the Mandibular length (mm).

\begin{tabular}{lccccc}
\hline \multirow{2}{*}{ Variable } & \multicolumn{2}{c}{ MARA } & & \multicolumn{2}{c}{ Edgewise } \\
\cline { 2 - 3 } \cline { 5 - 6 } Females & Males & & Females & Males \\
Arithmetic mean & 4.04 & 7.49 & & 7.12 & 6.14 \\
Standard deviation & 4.45 & 4.60 & & 5.90 & 6.38 \\
Standard error mean & 1.00 & 0.84 & & 1.32 & 1.15 \\
Upper 95\% CL & 6.12 & 9.21 & & 9.88 & 8.48 \\
Lower 95\% CL & 1.95 & 5.77 & & 4.35 & 3.80 \\
Sample size & 20 & 30 & & 20 & 31 \\
Variance & 19.84 & 21.15 & 34.86 & 40.71 \\
Skewness & -0.61 & -0.48 & 0.67 & 0.33 \\
Kurtosis & -0.13 & -0.28 & 1.03 & 0.58 \\
Number missing & 0 & 1 & 0 & 0 \\
T-test (one-sample) & 4.05 & 8.92 & 5.39 & 5.35 \\
Probability value & 0.0007 & $<0.0001$ & $<0.0001$ & $<0.0001$ \\
\hline
\end{tabular}


Table D-23. Descriptive statistics at the in-treatment changes for the Sella-to-A Point (mm).

\begin{tabular}{lccccc}
\hline \multirow{2}{*}{ Variable } & \multicolumn{2}{c}{ MARA } & & \multicolumn{2}{c}{ Edgewise } \\
\cline { 2 - 3 } \cline { 5 - 6 } & Females & Males & & Females & Males \\
Arithmetic mean & 0.16 & 3.26 & & 1.91 & 1.20 \\
Standard deviation & 1.74 & 2.73 & & 3.15 & 4.01 \\
Standard error mean & 0.39 & 0.49 & & 0.70 & 0.72 \\
Upper 95\% CL & 0.97 & 4.27 & & 3.38 & 2.67 \\
Lower 95\% CL & -0.65 & 2.26 & & 0.44 & -0.27 \\
Sample size & 20 & 31 & 20 & 31 \\
Variance & 3.03 & 7.45 & & 9.93 & 16.06 \\
Skewness & -1.81 & 0.65 & 0.69 & 0.88 \\
Kurtosis & 5.18 & -0.06 & 2.30 & 3.10 \\
Number missing & 0 & 0 & 0 & 0 \\
T-test (one-sample) & 0.41 & 6.66 & 2.71 & 1.66 \\
Probability value & 0.6854 & $<0.0001$ & 0.0139 & 0.1068 \\
\hline
\end{tabular}


Table D-24. Descriptive statistics at the in-treatment changes for the Sella-to-B Point (mm).

\begin{tabular}{lccccc}
\hline \multirow{2}{*}{ Variable } & \multicolumn{2}{c}{ MARA } & & \multicolumn{2}{c}{ Edgewise } \\
\cline { 2 - 3 } \cline { 5 - 6 } & & & & & \\
Females & Males & & & \\
Arithmetic mean & 4.74 & 8.27 & & 5.11 & 3.46 \\
Standard deviation & 2.44 & 3.36 & & 4.58 & 5.61 \\
Standard error mean & 0.55 & 0.60 & & 1.02 & 1.01 \\
Upper 95\% CL & 5.88 & 9.50 & & 7.25 & 5.52 \\
Lower 95\% CL & 3.59 & 7.04 & & 2.96 & 1.41 \\
Sample size & 20 & 31 & & 20 & 31 \\
Variance & 5.95 & 11.26 & & 21.01 & 31.51 \\
Skewness & 1.15 & 0.41 & 0.14 & 0.85 \\
Kurtosis & 2.79 & -0.33 & 0.04 & 2.66 \\
Number missing & 0 & 0 & 0 & 0 \\
T-test (one-sample) & 8.68 & 13.72 & 4.98 & 3.44 \\
Probability value & $<0.0001$ & $<0.0001$ & $<0.0001$ & 0.0017 \\
\hline
\end{tabular}


Table D-25. Descriptive statistics at the in-treatment changes for the Overbite (mm).

\begin{tabular}{lccccc}
\hline \multirow{2}{*}{ Variable } & \multicolumn{2}{c}{ MARA } & & \multicolumn{2}{c}{ Edgewise } \\
\cline { 2 - 3 } \cline { 5 - 6 } & Females & Males & & Females & Males \\
Arithmetic mean & -1.97 & -2.01 & & -2.18 & -2.08 \\
Standard deviation & 1.79 & 1.68 & & 2.54 & 1.87 \\
Standard error mean & 0.40 & 0.30 & & 0.57 & 0.34 \\
Upper 95\% CL & -1.13 & -1.39 & & -0.99 & -1.40 \\
Lower 95\% CL & -2.80 & -2.63 & & -3.36 & -2.77 \\
Sample size & 20 & 31 & & 20 & 31 \\
Variance & 3.22 & 2.82 & & 6.46 & 3.49 \\
Skewness & 0.26 & 0.36 & -0.16 & 0.13 \\
Kurtosis & -0.09 & -0.54 & 0.56 & -0.76 \\
Number missing & 0 & 0 & 0 & 0 \\
T-test (one-sample) & -4.90 & -6.66 & -3.83 & -6.21 \\
Probability value & $<0.0001$ & $<0.0001$ & 0.0011 & $<0.0001$ \\
\hline
\end{tabular}


Table D-26. Descriptive statistics at the in-treatment changes for the Overjet (mm).

\begin{tabular}{lccccc}
\hline \multirow{2}{*}{ Variable } & \multicolumn{2}{c}{ MARA } & & \multicolumn{2}{c}{ Edgewise } \\
\cline { 2 - 3 } \cline { 5 - 6 } & Females & Males & & Females & Males \\
Arithmetic mean & -3.17 & -2.57 & & -3.89 & -4.99 \\
Standard deviation & 1.86 & 1.75 & & 2.62 & 2.89 \\
Standard error mean & 0.42 & 0.31 & & 0.59 & 0.52 \\
Upper 95\% CL & -2.29 & -1.93 & & -2.66 & -3.93 \\
Lower 95\% CL & -4.04 & -3.21 & & -5.12 & -6.05 \\
Sample size & 20 & 31 & & 20 & 31 \\
Variance & 3.47 & 3.05 & & 6.87 & 8.35 \\
Skewness & -0.53 & -0.48 & -0.08 & -0.61 \\
Kurtosis & -0.90 & 0.11 & -0.22 & -0.14 \\
Number missing & 0 & 0 & 0 & 0 \\
T-test (one-sample) & -7.60 & -8.20 & -6.64 & -9.61 \\
Probability value & $<0.0001$ & $<0.0001$ & $<0.0001$ & $<0.0001$ \\
\hline
\end{tabular}


Table D-27. Descriptive statistics at the in-treatment changes for the A Pt to NaPerp (mm).

\begin{tabular}{lccccc}
\hline \multirow{2}{*}{ Variable } & \multicolumn{2}{c}{ MARA } & & \multicolumn{2}{c}{ Edgewise } \\
\cline { 2 - 3 } \cline { 5 - 6 } & Females & Males & & Females & Males \\
Arithmetic mean & -0.85 & -2.31 & & -1.87 & -2.11 \\
Standard deviation & 3.43 & 2.51 & & 1.38 & 2.81 \\
Standard error mean & 0.77 & 0.45 & & 0.31 & 0.50 \\
Upper 95\% CL & 0.76 & -1.39 & & -1.22 & -1.08 \\
Lower 95\% CL & -2.46 & -3.23 & -2.52 & -3.14 \\
Sample size & 20 & 31 & & 20 & 31 \\
Variance & 11.79 & 6.29 & & 1.91 & 7.89 \\
Skewness & 0.60 & 0.16 & 0.51 & -0.44 \\
Kurtosis & 0.50 & -0.35 & 0.35 & -0.28 \\
Number missing & 0 & 0 & 0 & 0 \\
T-test (one-sample) & -1.11 & -5.12 & -6.05 & -4.18 \\
Probability value & 0.2821 & $<0.0001$ & $<0.0001$ & 0.0002 \\
\hline
\end{tabular}


Table D-28. Descriptive statistics at the in-treatment changes for the B Pt to NaPerp (mm).

\begin{tabular}{lccccc}
\hline \multirow{2}{*}{ Variable } & \multicolumn{2}{c}{ MARA } & & \multicolumn{2}{c}{ Edgewise } \\
\cline { 2 - 3 } \cline { 5 - 6 } & Females & Males & & Females & Males \\
Arithmetic mean & 2.10 & -0.10 & & 0.31 & -1.51 \\
Standard deviation & 5.90 & 4.33 & & 2.58 & 4.35 \\
Standard error mean & 1.32 & 0.78 & & 0.58 & 0.78 \\
Upper 95\% CL & 4.86 & 1.49 & & 1.52 & 0.09 \\
Lower 95\% CL & -0.66 & -1.69 & & -0.90 & -3.10 \\
Sample size & 20 & 31 & & 20 & 31 \\
Variance & 34.78 & 18.71 & & 6.63 & 18.91 \\
Skewness & 0.59 & 0.12 & -0.63 & -0.08 \\
Kurtosis & 1.45 & -0.59 & -0.62 & -0.09 \\
Number missing & 0 & 0 & 0 & 0 \\
T-test (one-sample) & 1.59 & -0.13 & 0.54 & -1.93 \\
Probability value & 0.1278 & 0.8984 & 0.5966 & 0.0627 \\
\hline
\end{tabular}


APPENDIX E.

MEAN IN-TREATMENT CHANGES, BY TREATMENT AND SEX, OF THE CEPHALOMETRIC VARIABLES. 


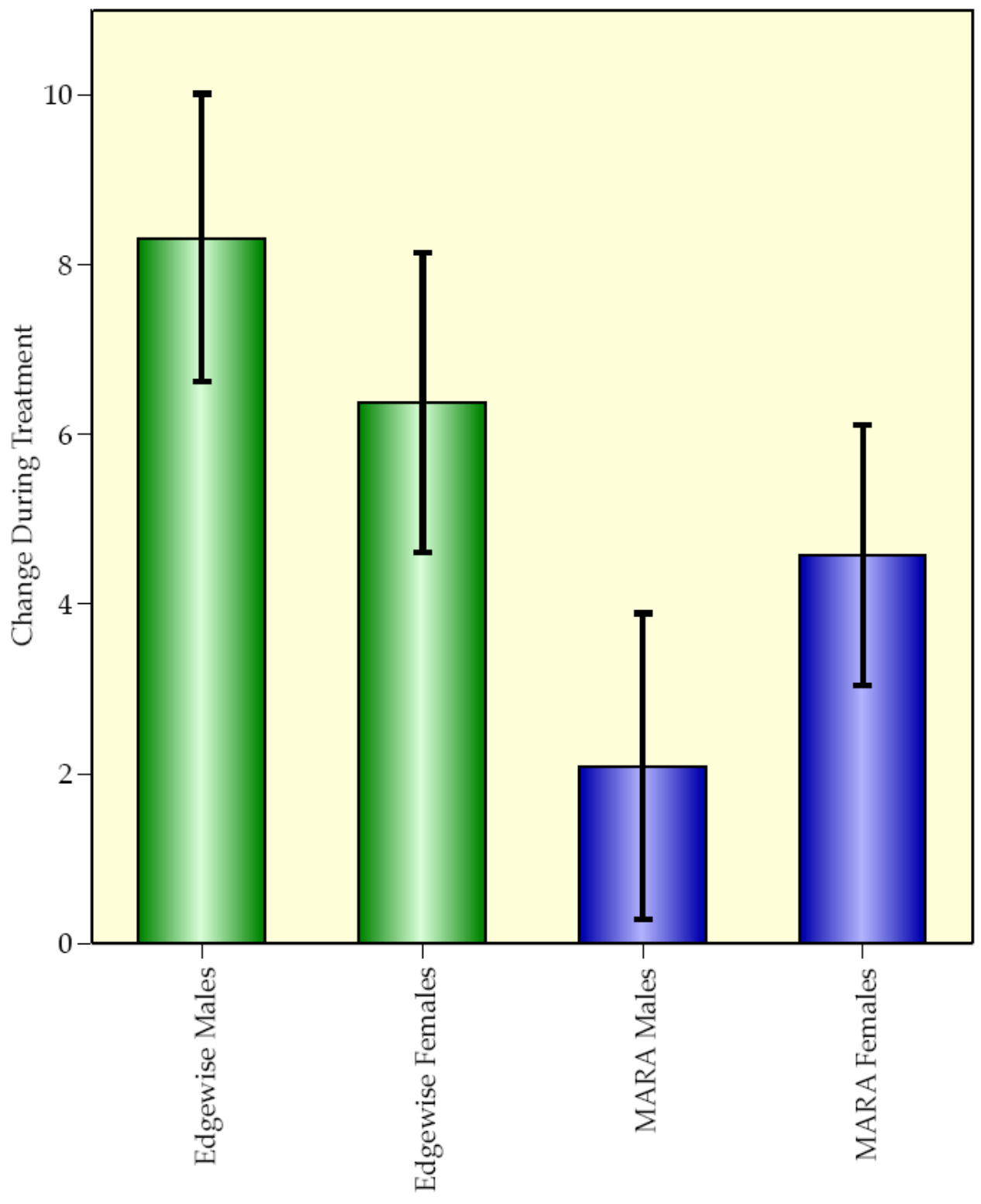

Figure E-1. Mean in-treatment changes in the Z Angle, by treatment and by sex. Error bars are \pm one standard error of the mean. 


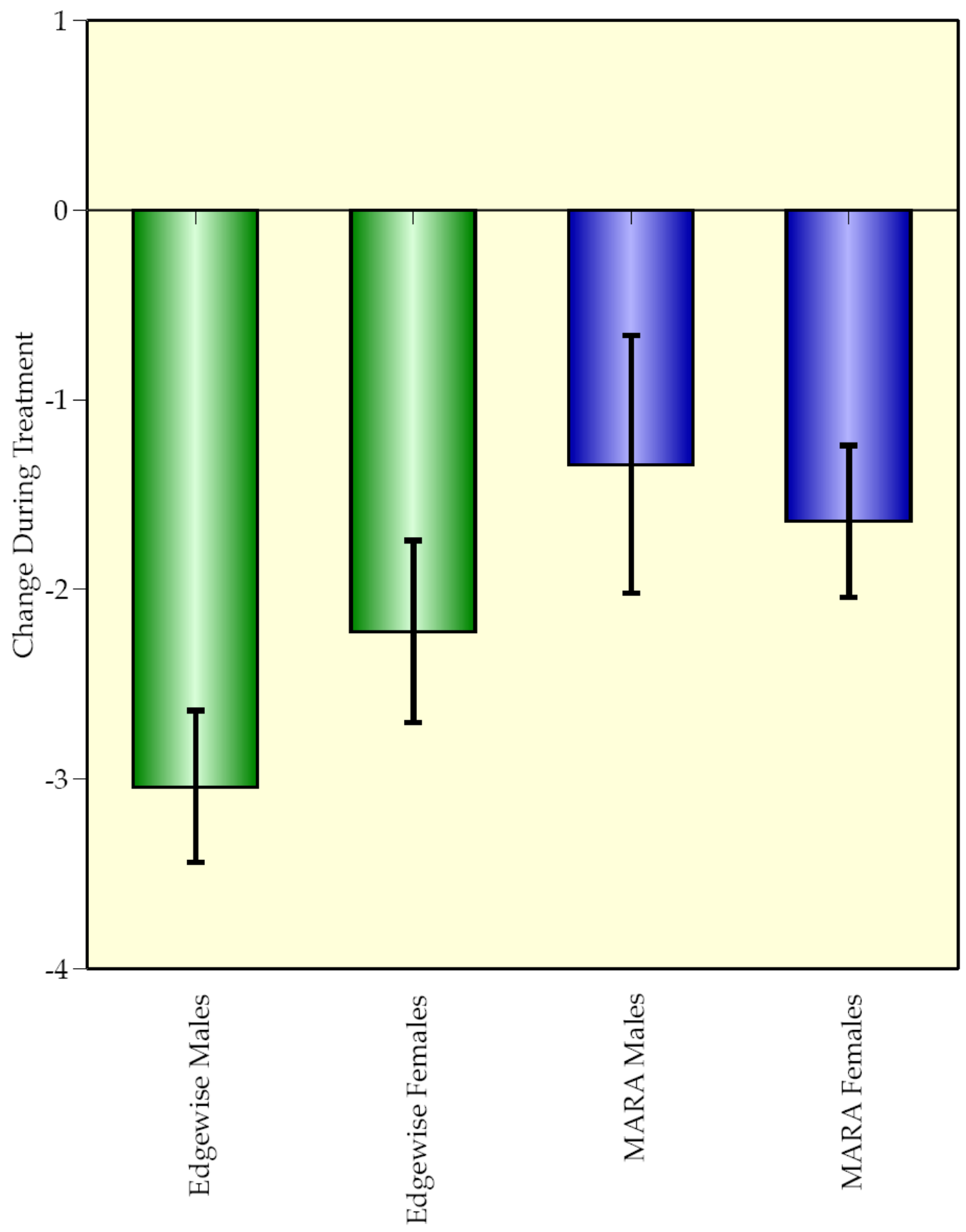

Figure E-2. Mean in-treatment changes in the E Plane, by treatment and by sex. Error bars are \pm one standard error of the mean. 


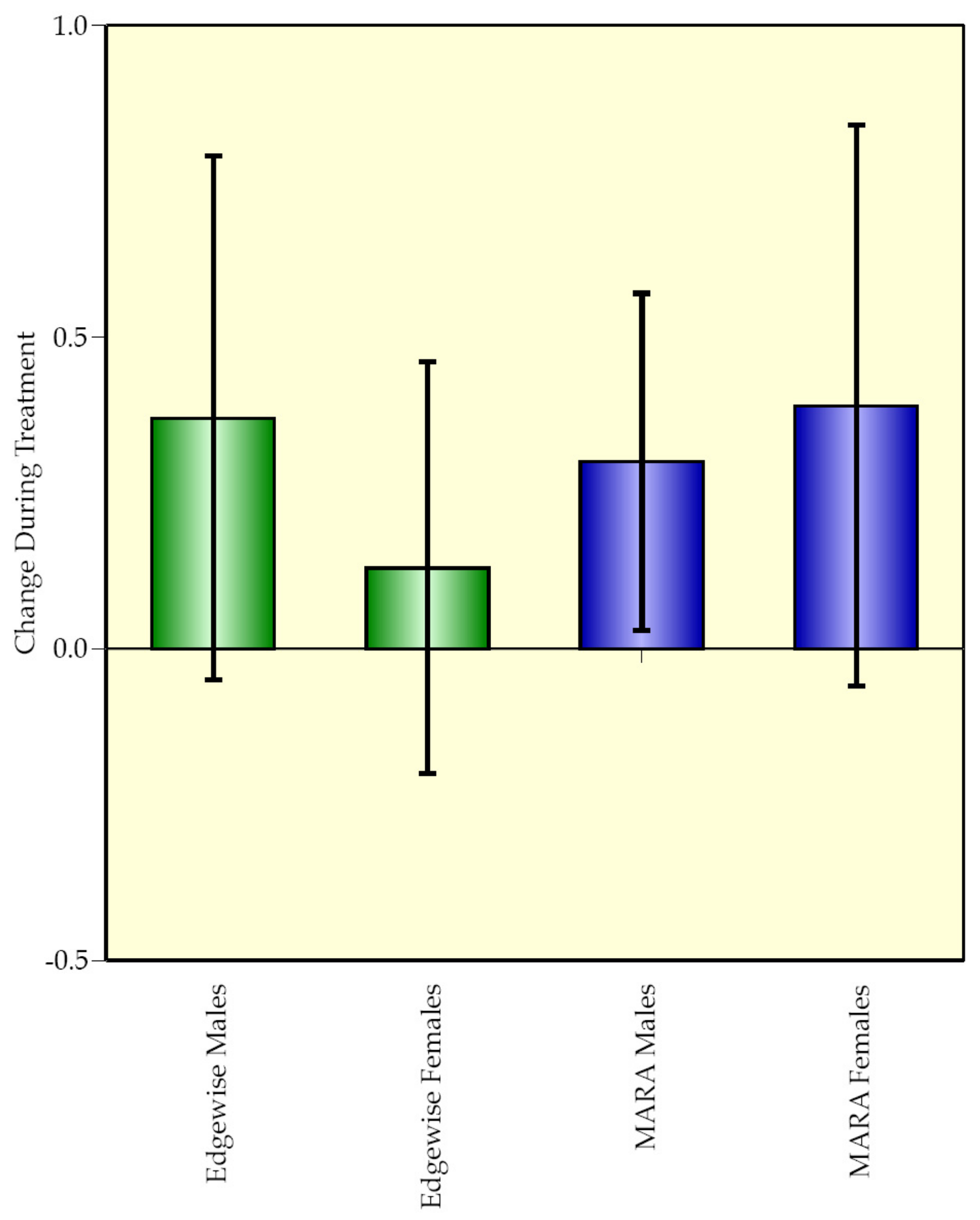

Figure E-3. Mean in-treatment change in the Y-axis, by treatment and by sex. Error bars are \pm one standard error of the mean. 


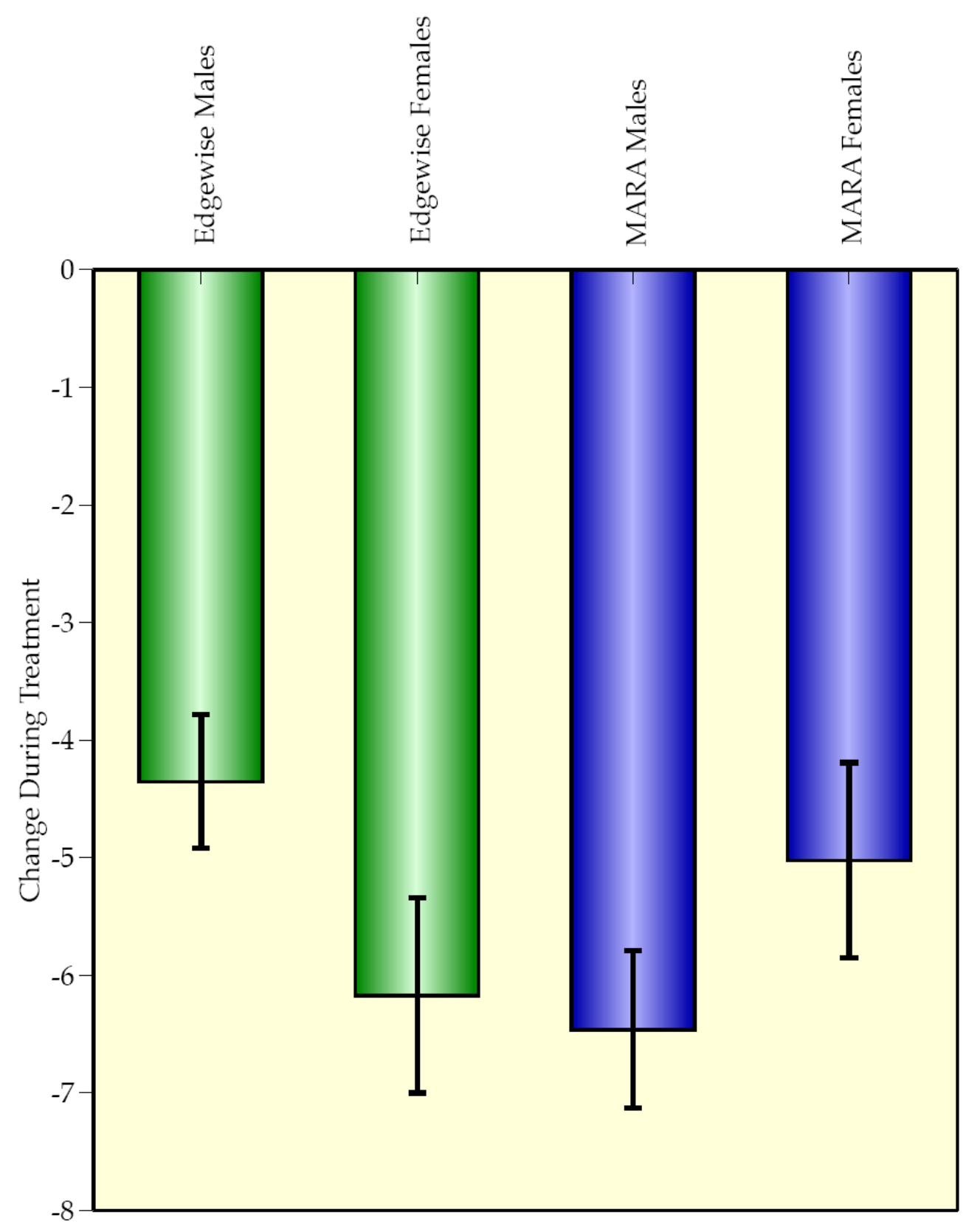

Figure E-4. Mean in-treatment changes for the NaPg Angle, by treatment and by sex. Error bars are \pm one standard error of the mean. 


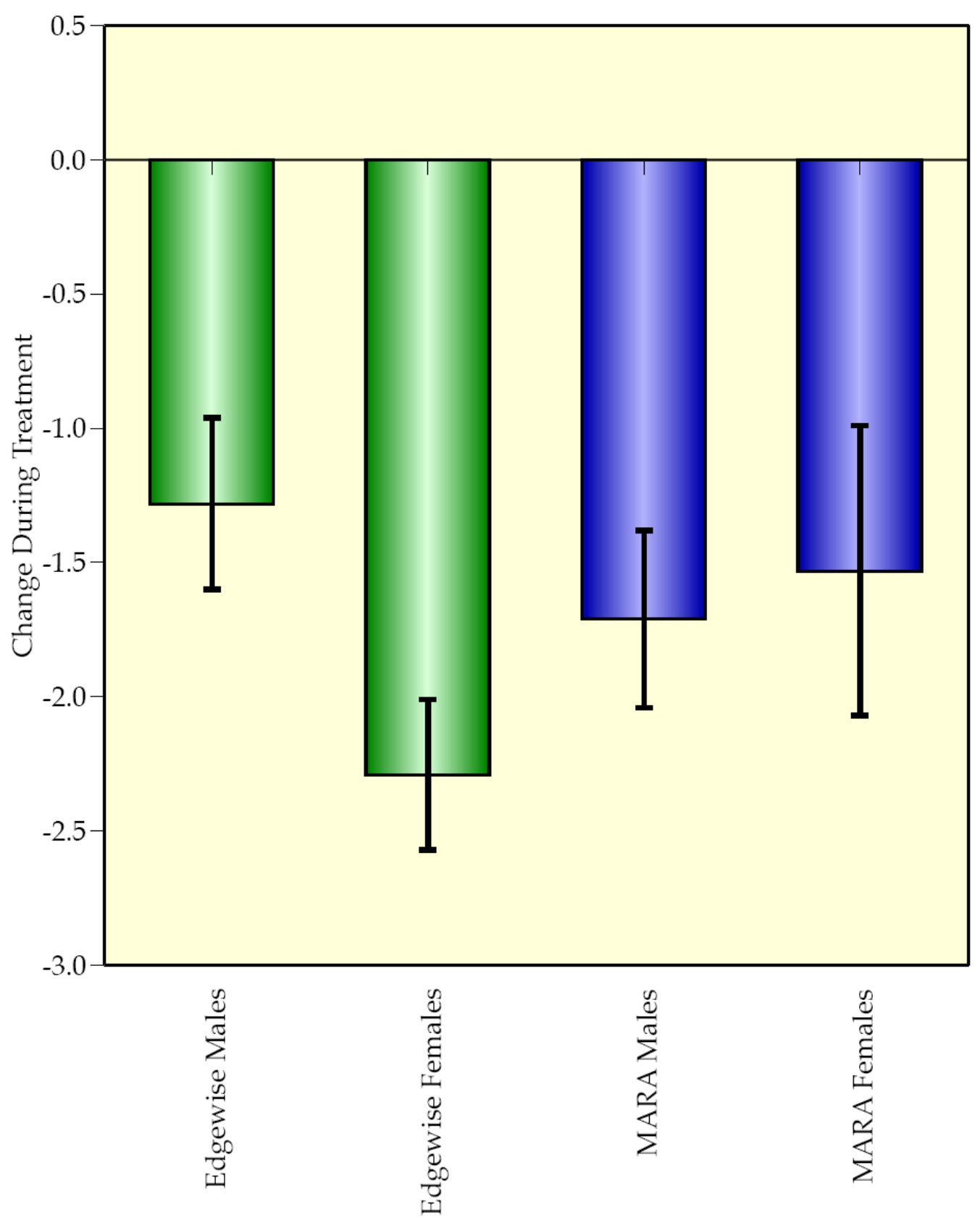

Figure E-5. Mean in-treatment changes in the SNA Angle, by treatment and by sex. Error bars are \pm one standard error of the mean. 


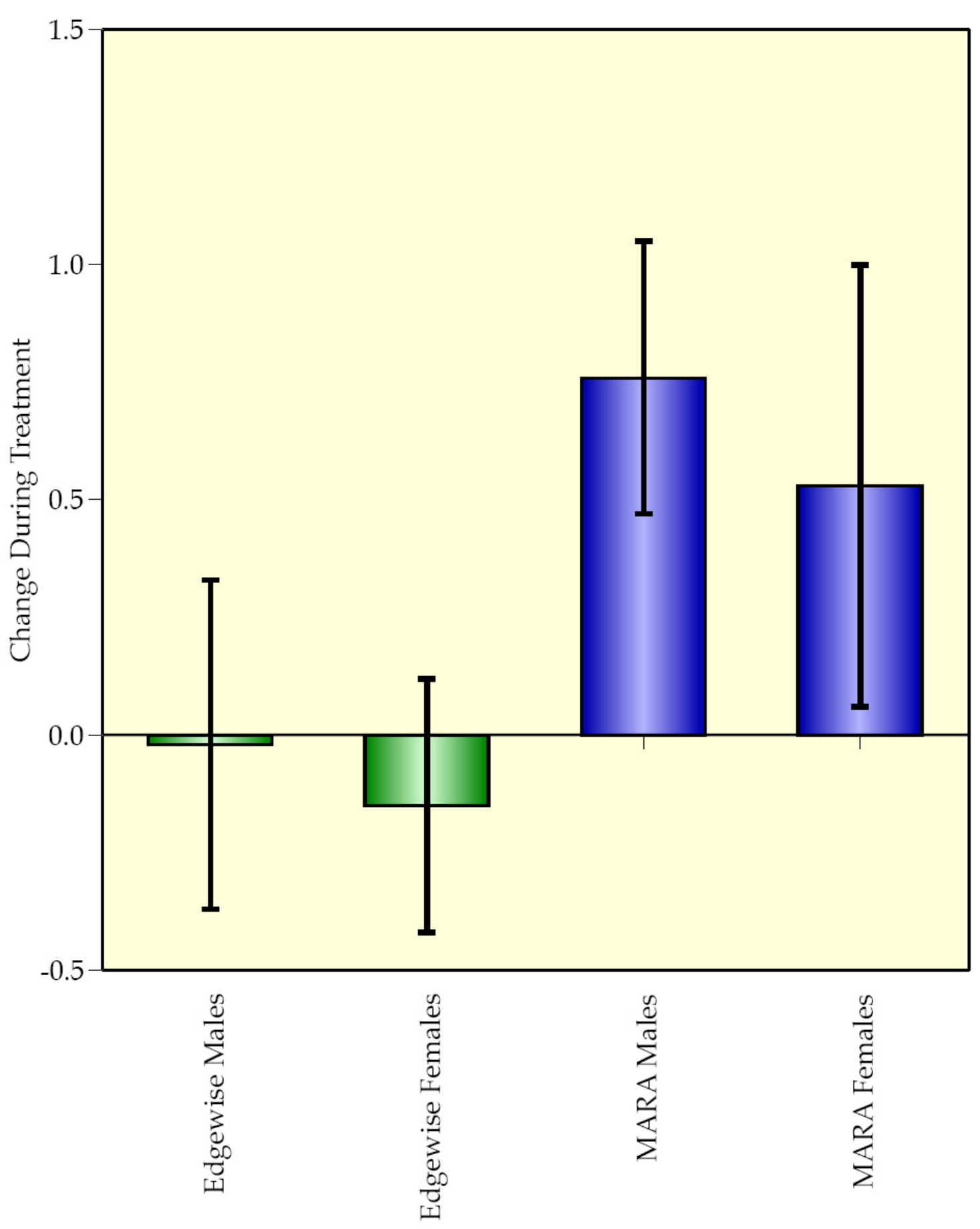

Figure E-6. Mean in-treatment changes in the SNB Angle, by treatment and by sex. Error bars are \pm one standard error of the mean. 


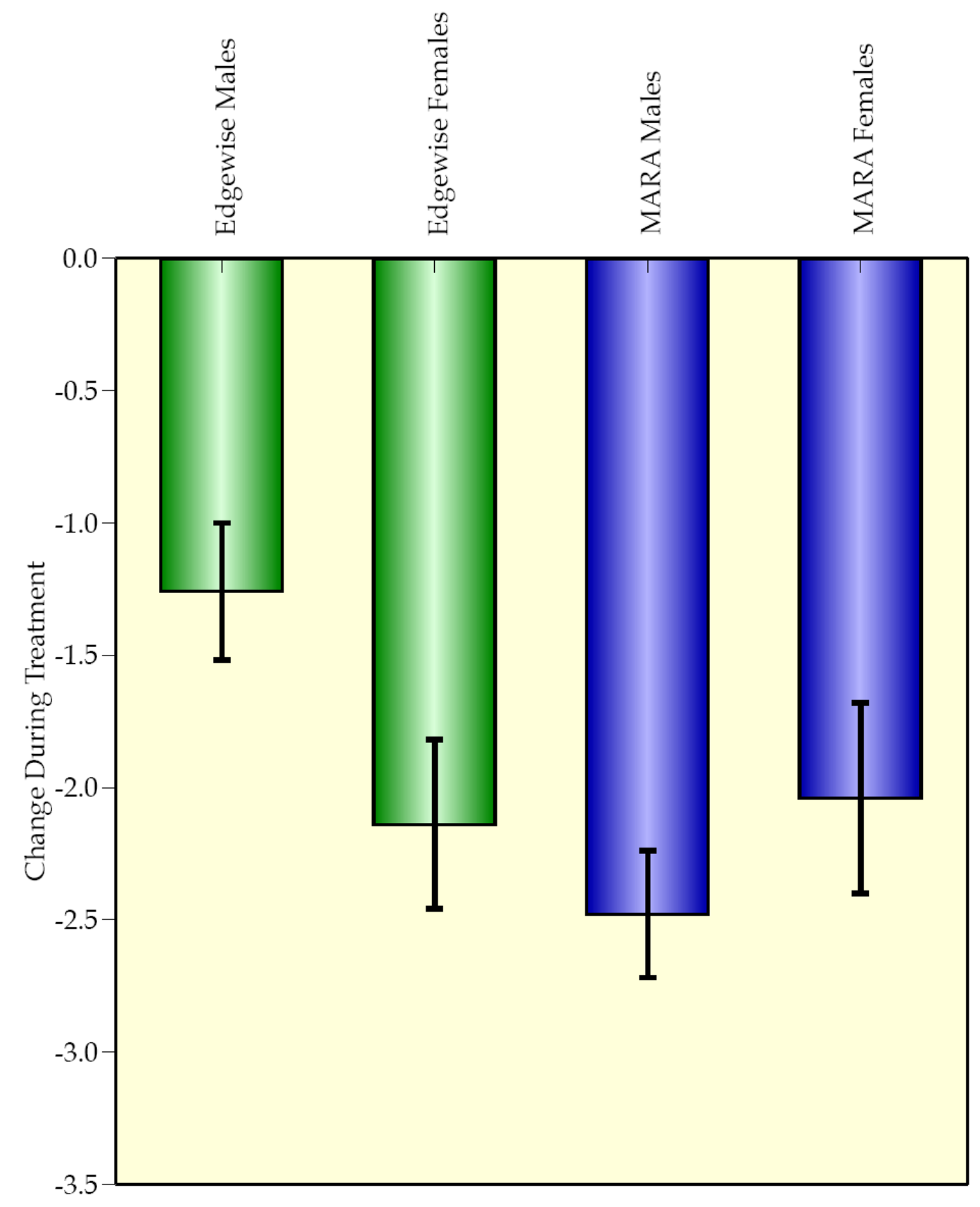

Figure E-7. Mean in-treatment changes in the ANB Angle, by treatment and by sex. Error bars are \pm one standard error of the mean. 


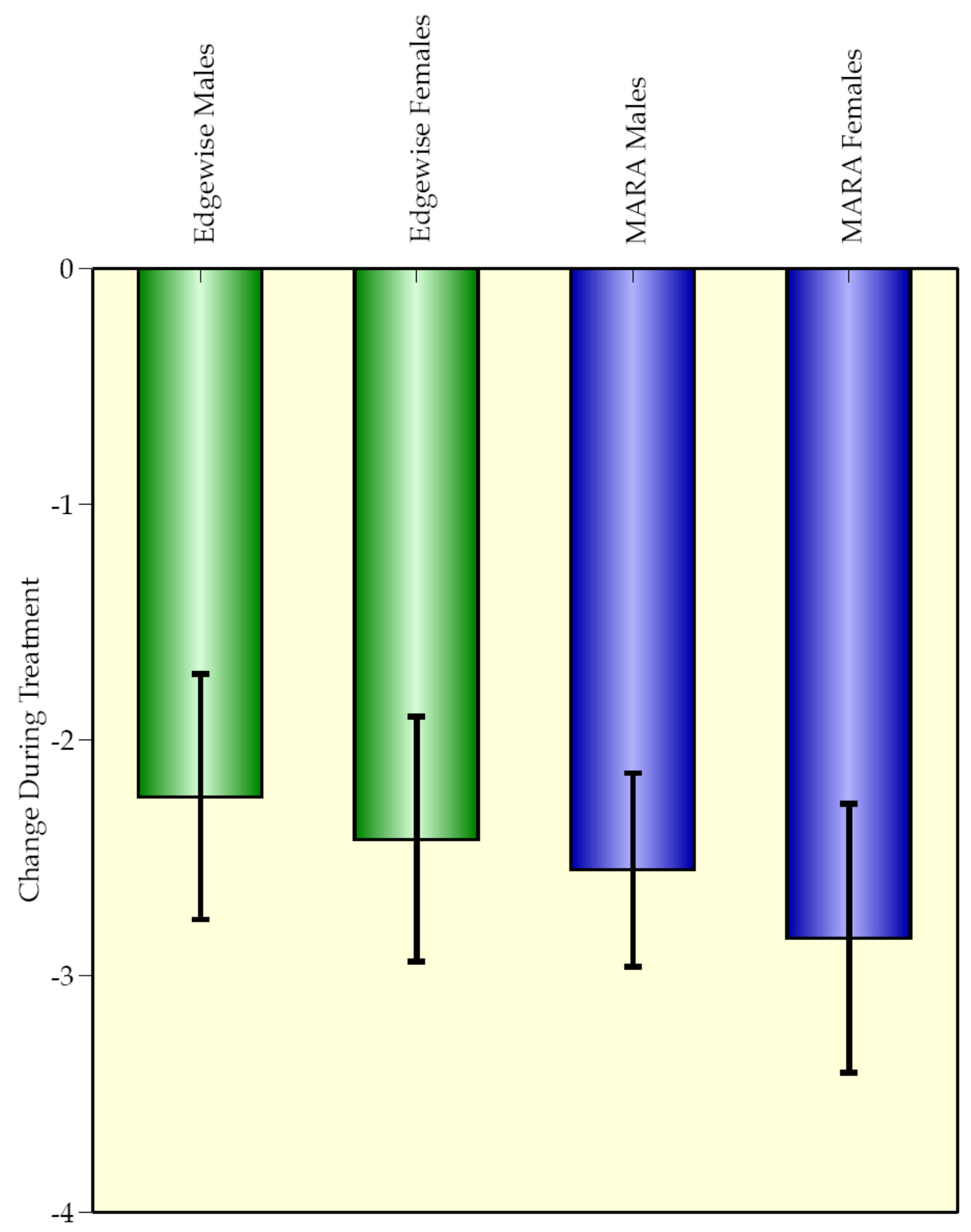

Figure E-7. Mean in-treatment changes in the AOBO, by treatment and by sex. Error bars are \pm one standard error of the mean. 


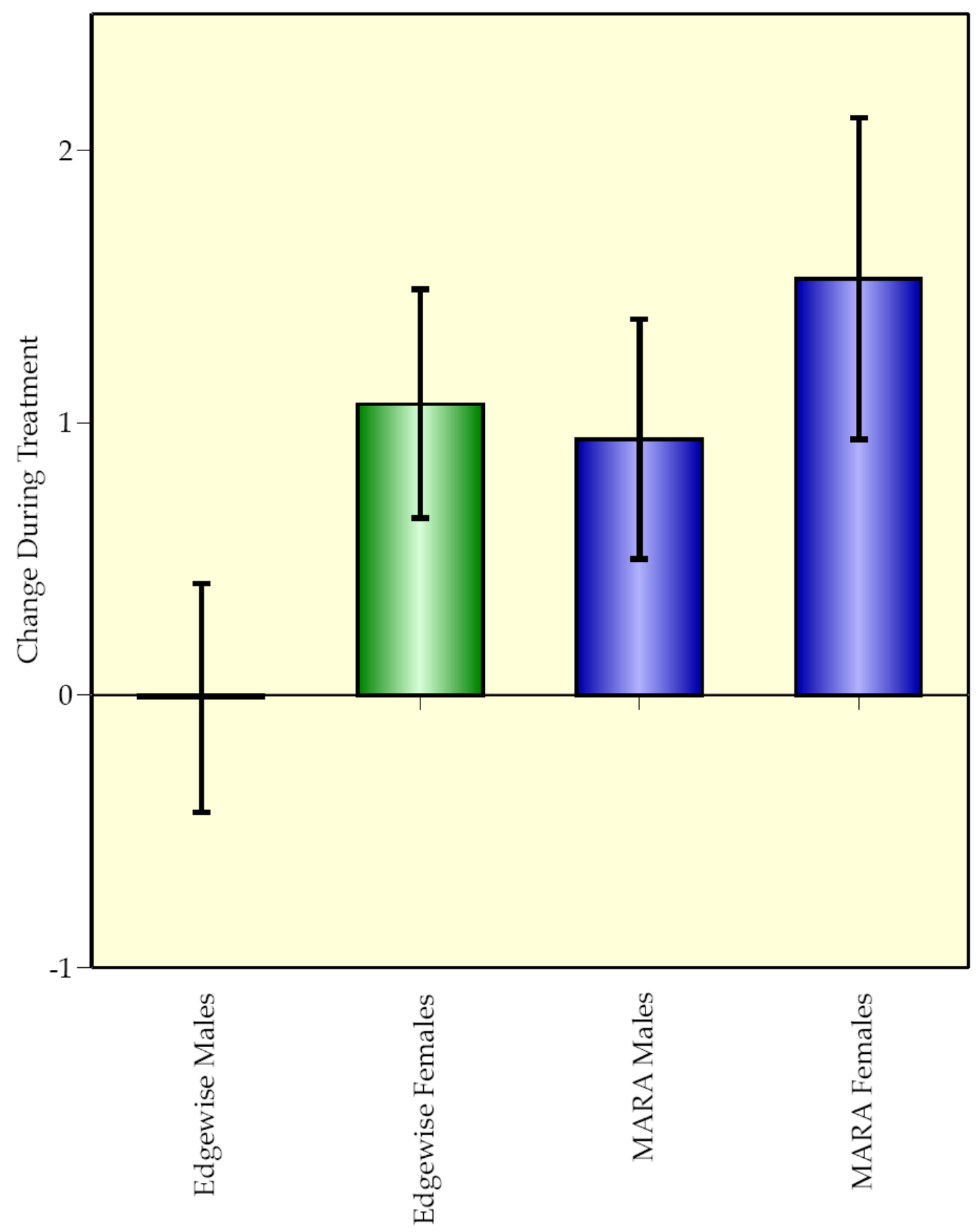

Figure E-8. Mean in-treatment changes in Facial angle, by treatment and by sex. Error bars are \pm one standard error of the mean. 


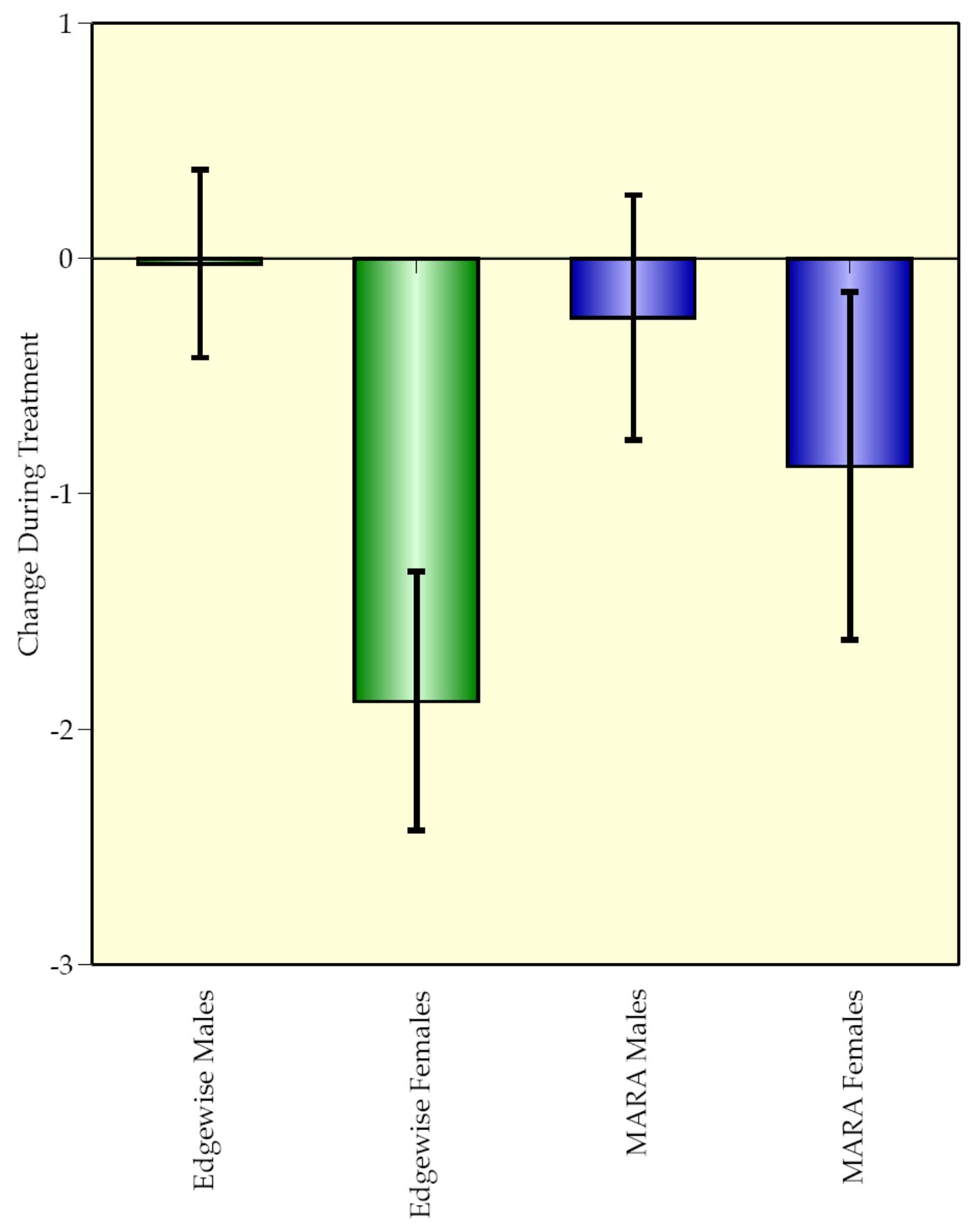

Figure E-9. Mean in-treatment changes for FMA, by treatment and by sex. Error bars are \pm one standard error of the mean. 


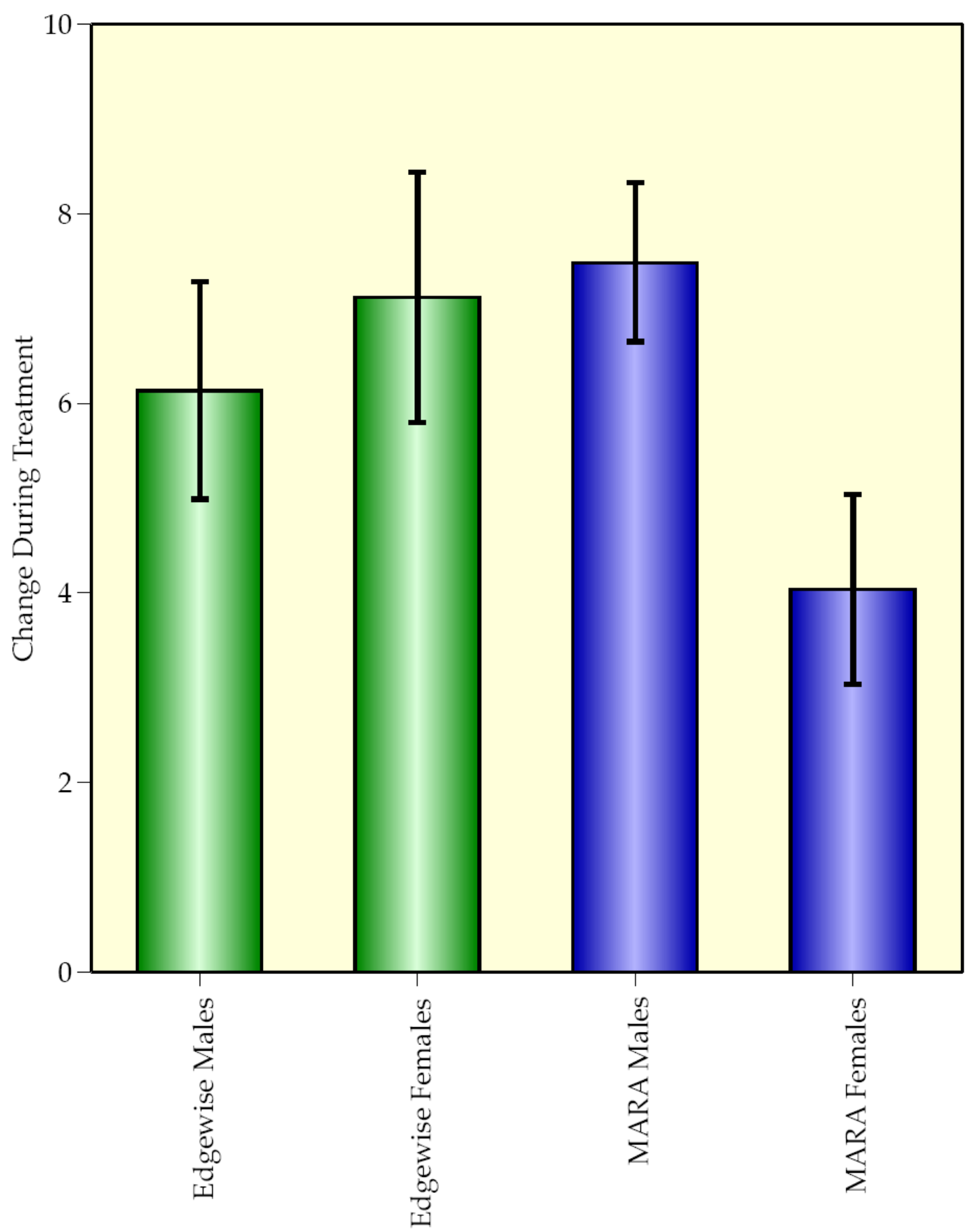

Figure E-10. Mean in-treatment changes for mandibular length, by treatment and by sex. Error bars are \pm one standard error of the mean. 


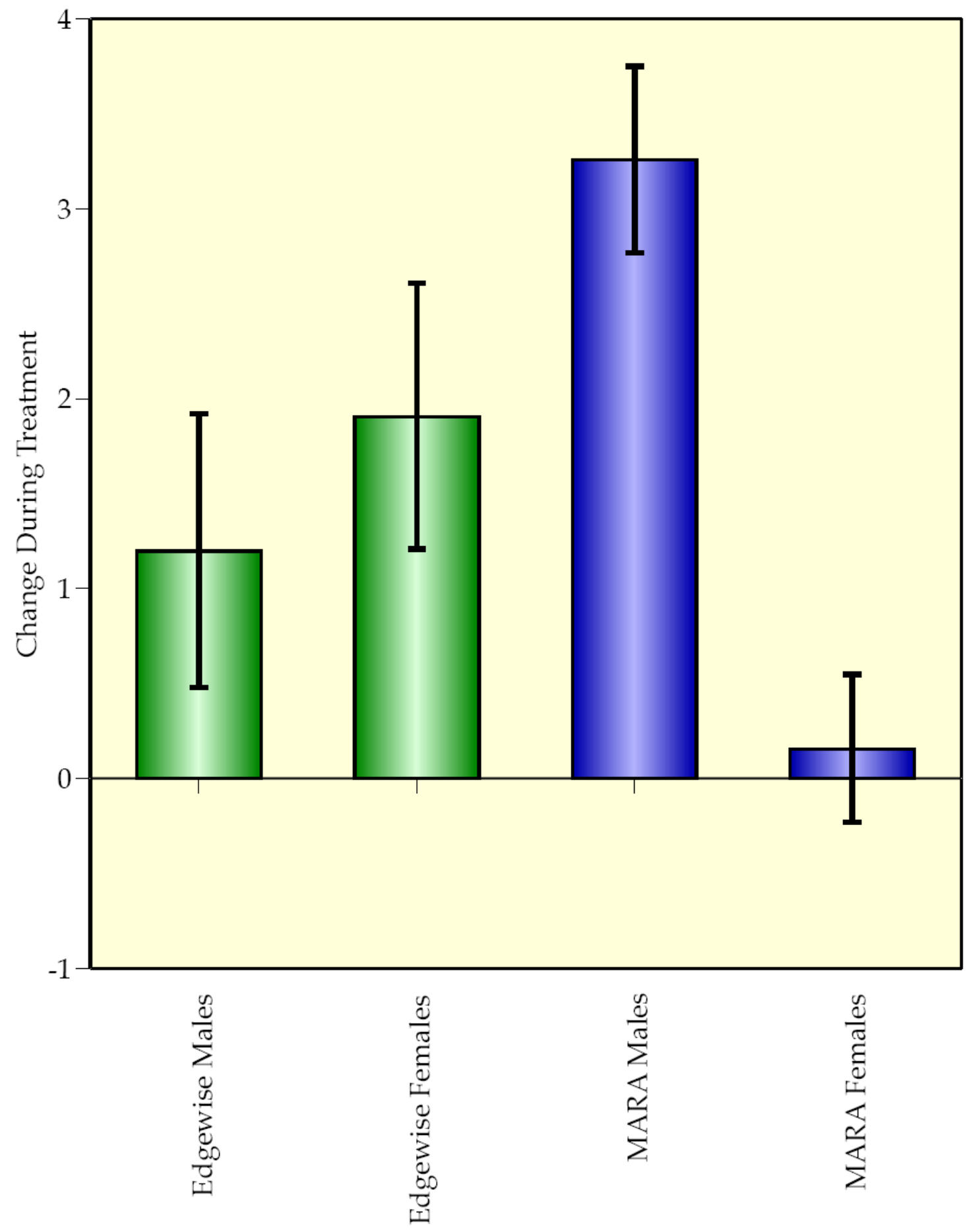

Figure E-11. Mean in-treatment changes in Sella-A Point length, by treatment and by sex. Error bars are \pm one standard error of the mean. 


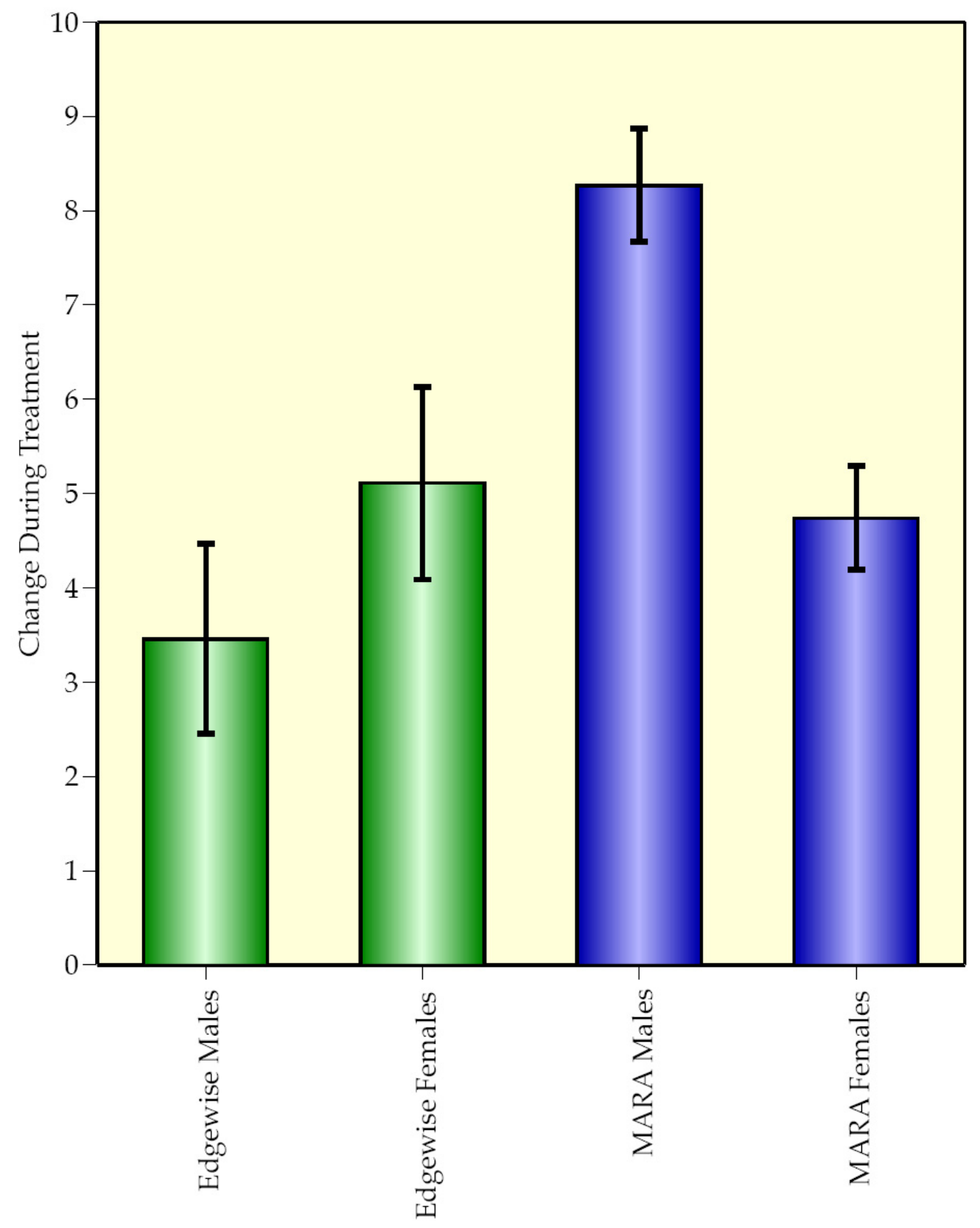

Figure E-12. Mean in-treatment changes in Sella-B Point length, by treatment and by sex. Error bars are \pm one standard error of the mean. 


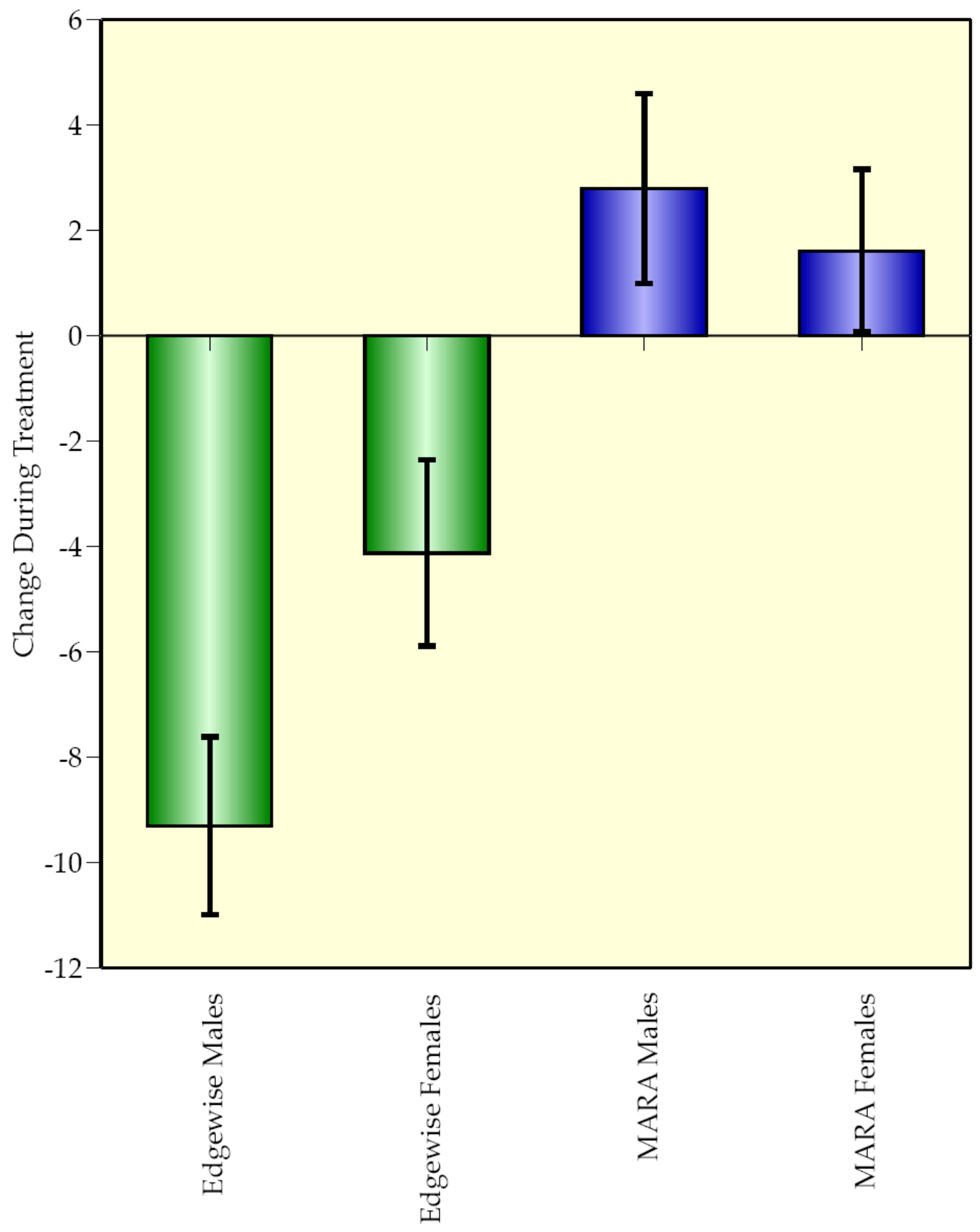

Figure E-13. Mean in-treatment changes in U1 to Sella Nasion Angle, by treatment and by sex. Error bars are \pm one standard error of the mean. 


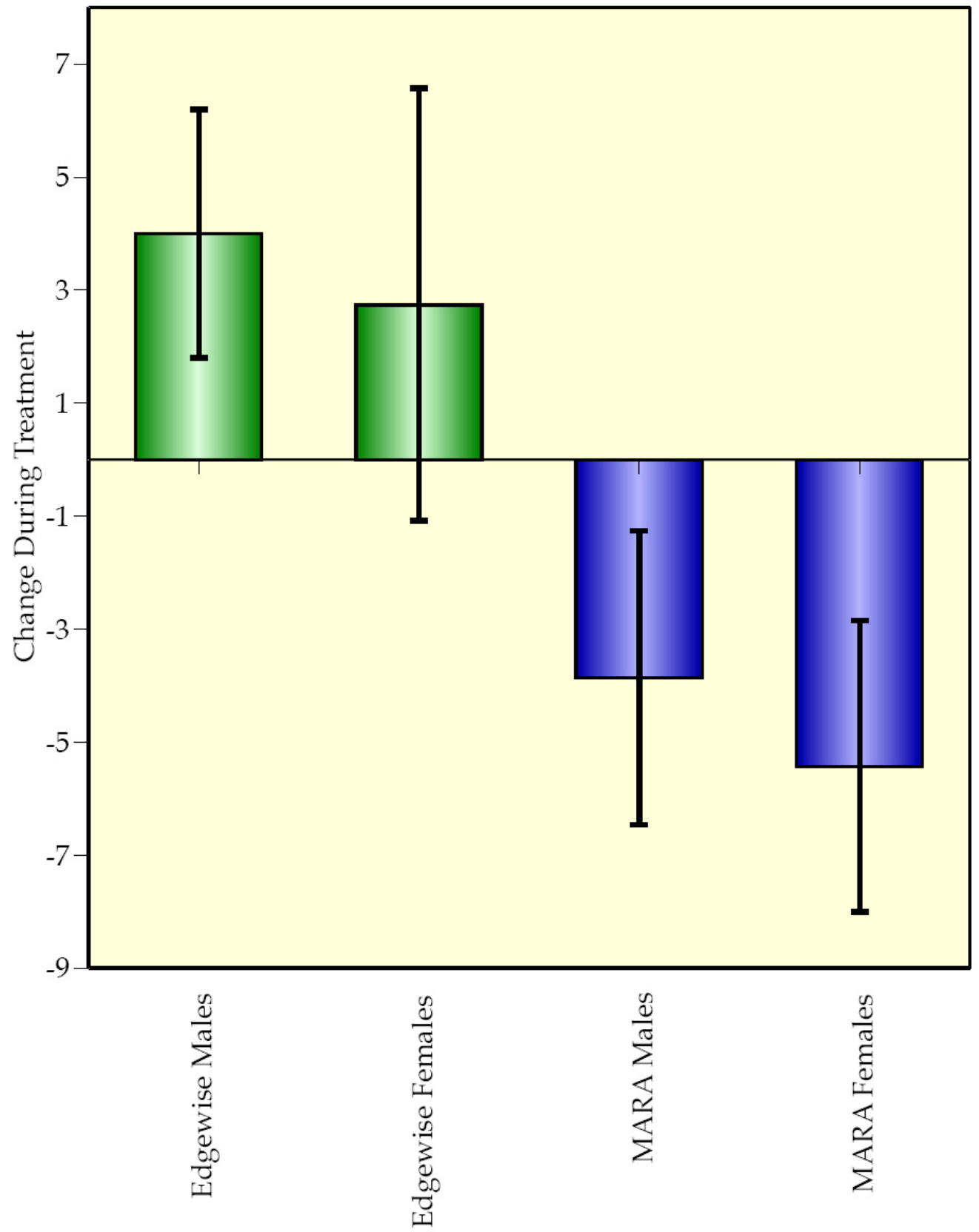

Figure E-14. Mean in-treatment changes in Interincisal Angle, by treatment and by sex. Error bars are \pm one standard error of the mean. 


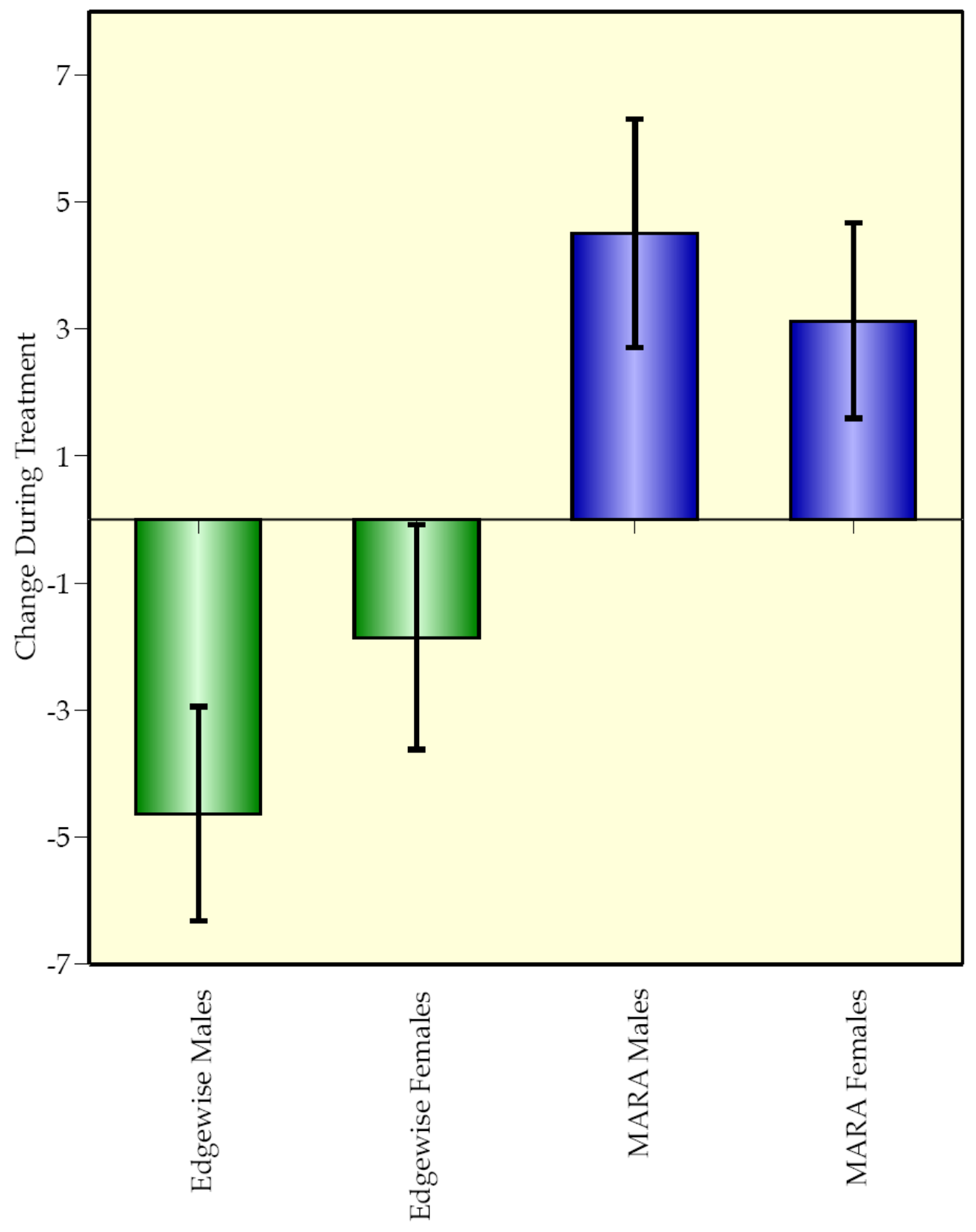

Figure E-15. Mean in-treatment changes in U1 to Na A Point Angle, by treatment and by sex. Error bars are \pm one standard error of the mean. 


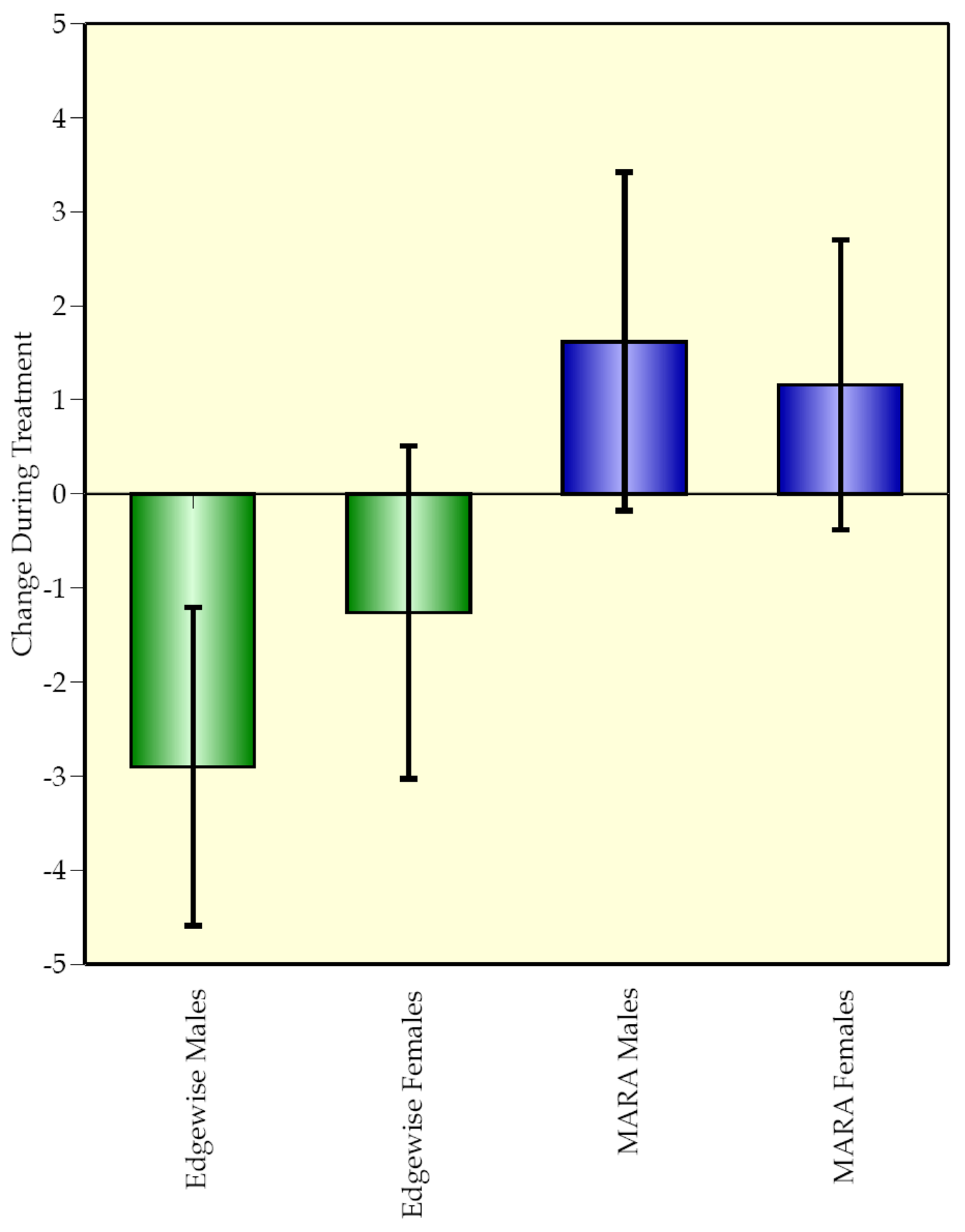

Figure E-16. Mean in-treatment changes in U1 to Na A Point (mm), by treatment and by sex. Error bars are \pm one standard error of the mean. 


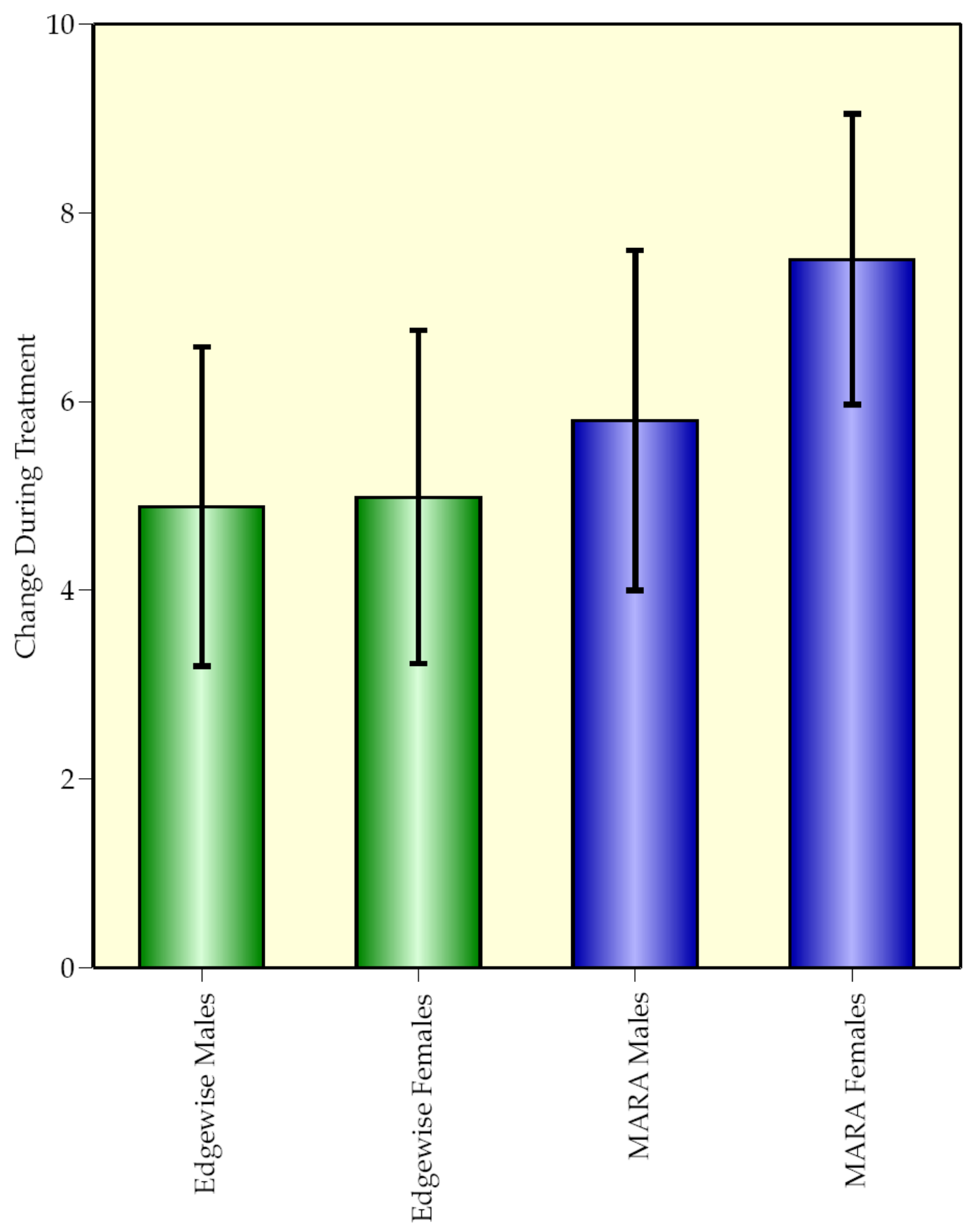

Figure E-17. Mean in-treatment changes in L1 to A Pogonion Angle, by treatment and by sex. Error bars are \pm one standard error of the mean. 


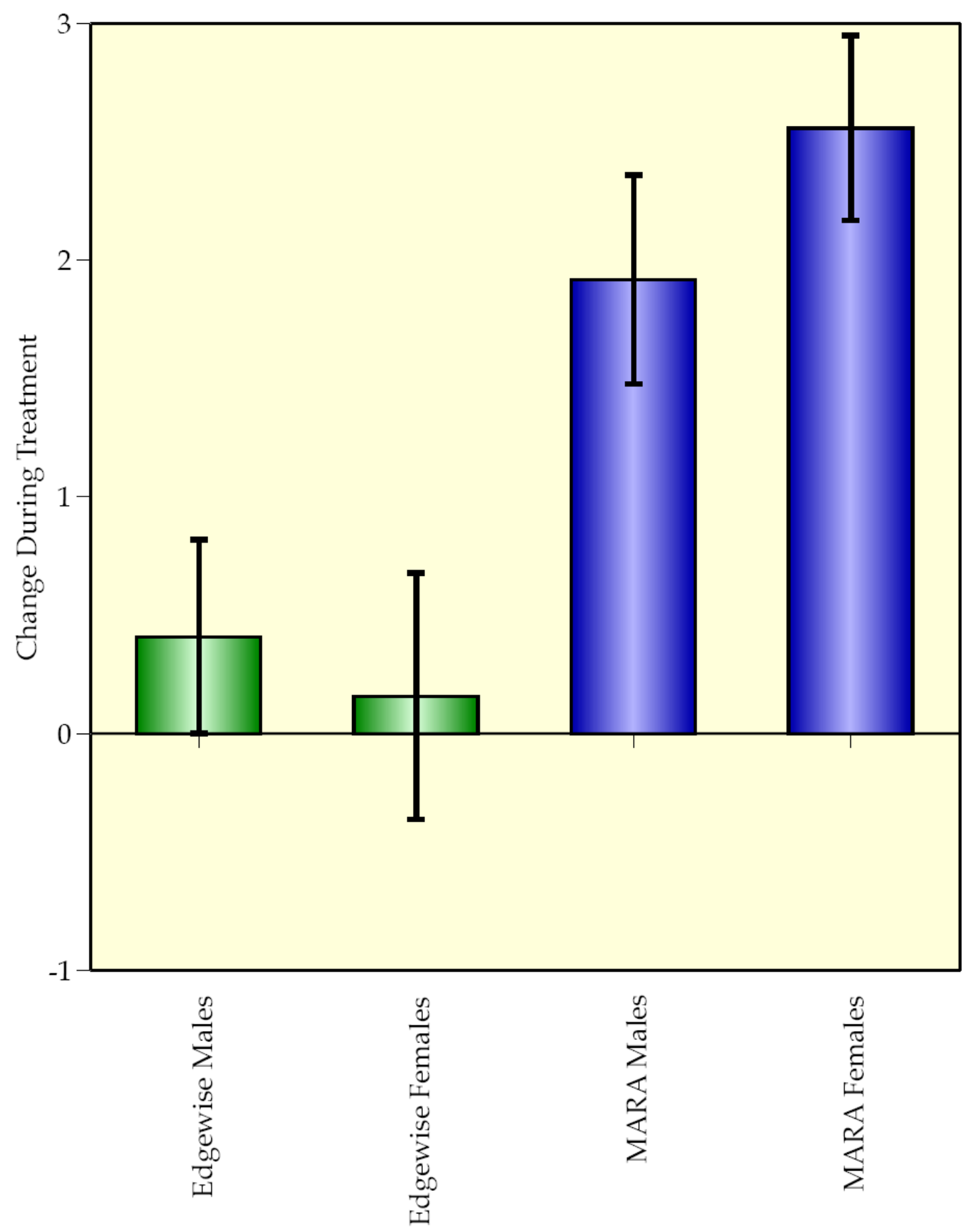

Figure E-18. Mean in-treatment changes in L1 to A Pogonion (mm), by treatment and by sex. Error bars are \pm one standard error of the mean. 


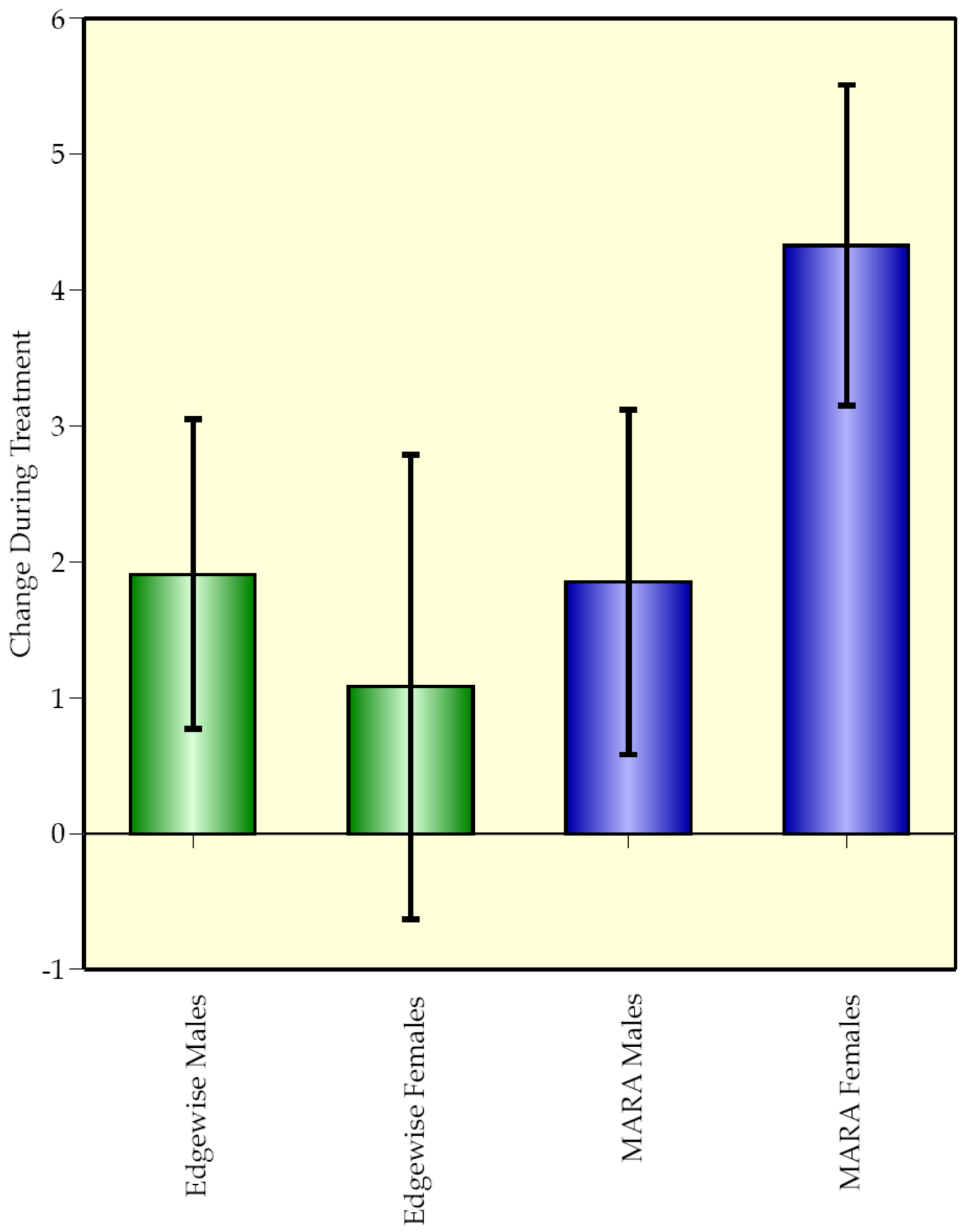

Figure E-19. Mean in-treatment changes in L1 to Nasion B Point Angle, by treatment and by sex. Error bars are \pm one standard error of the mean. 


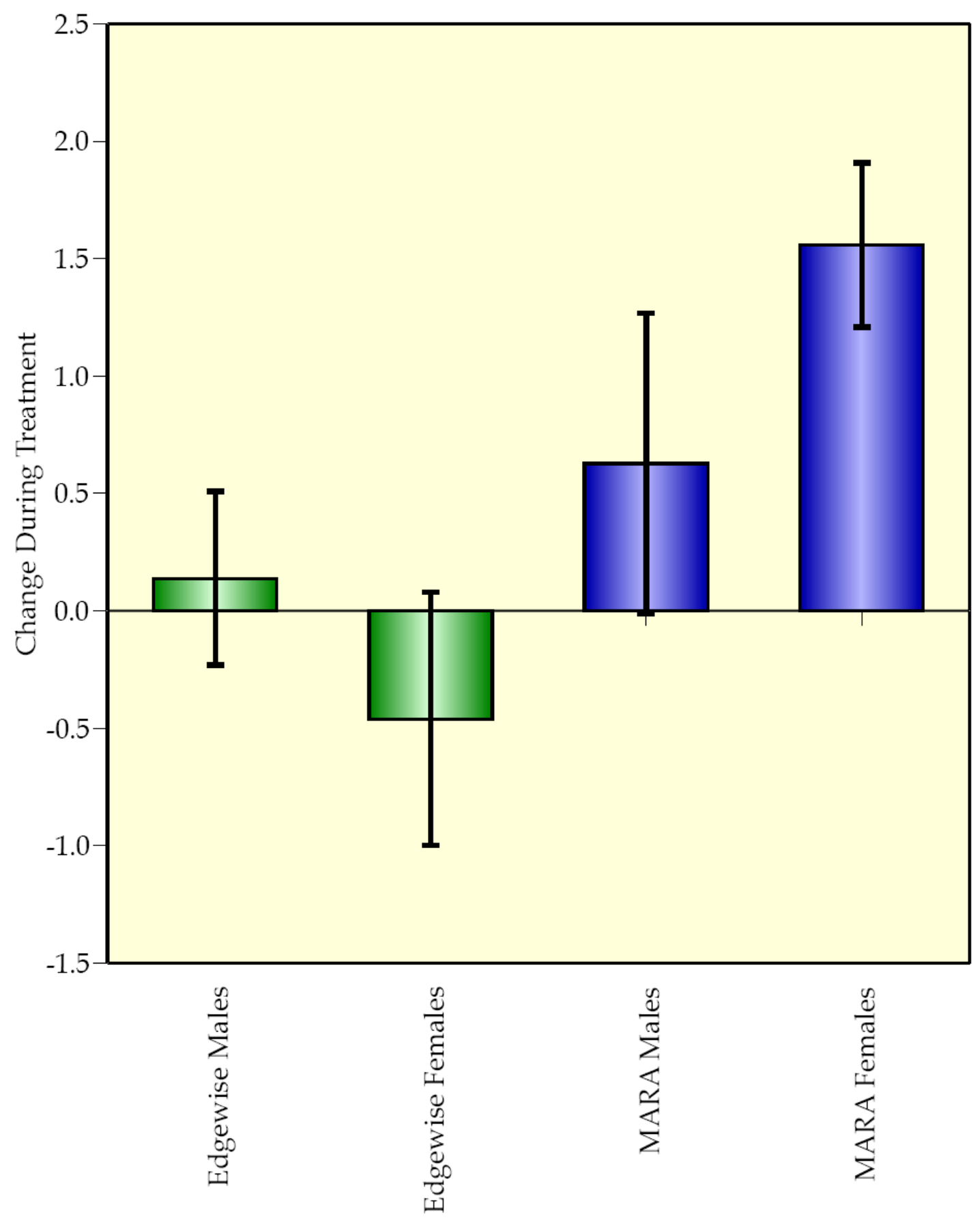

Figure E-20. Mean in-treatment changes in L1 to Nasion B Point (mm), by treatment and by sex. Error bars are \pm one standard error of the mean. 


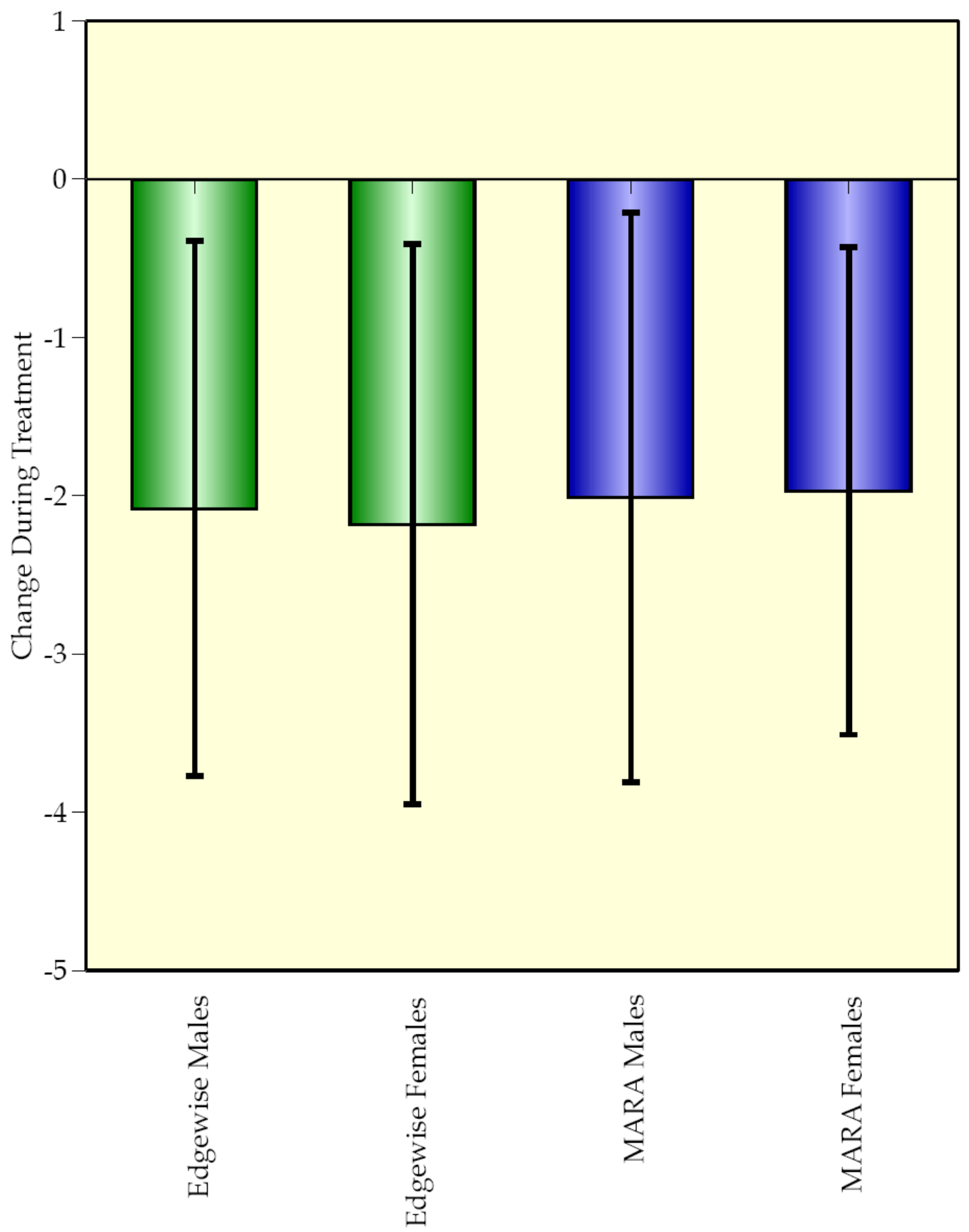

Figure E-21. Mean in-treatment changes in overbite, by treatment and by sex. Error bars are \pm one standard error of the mean. 


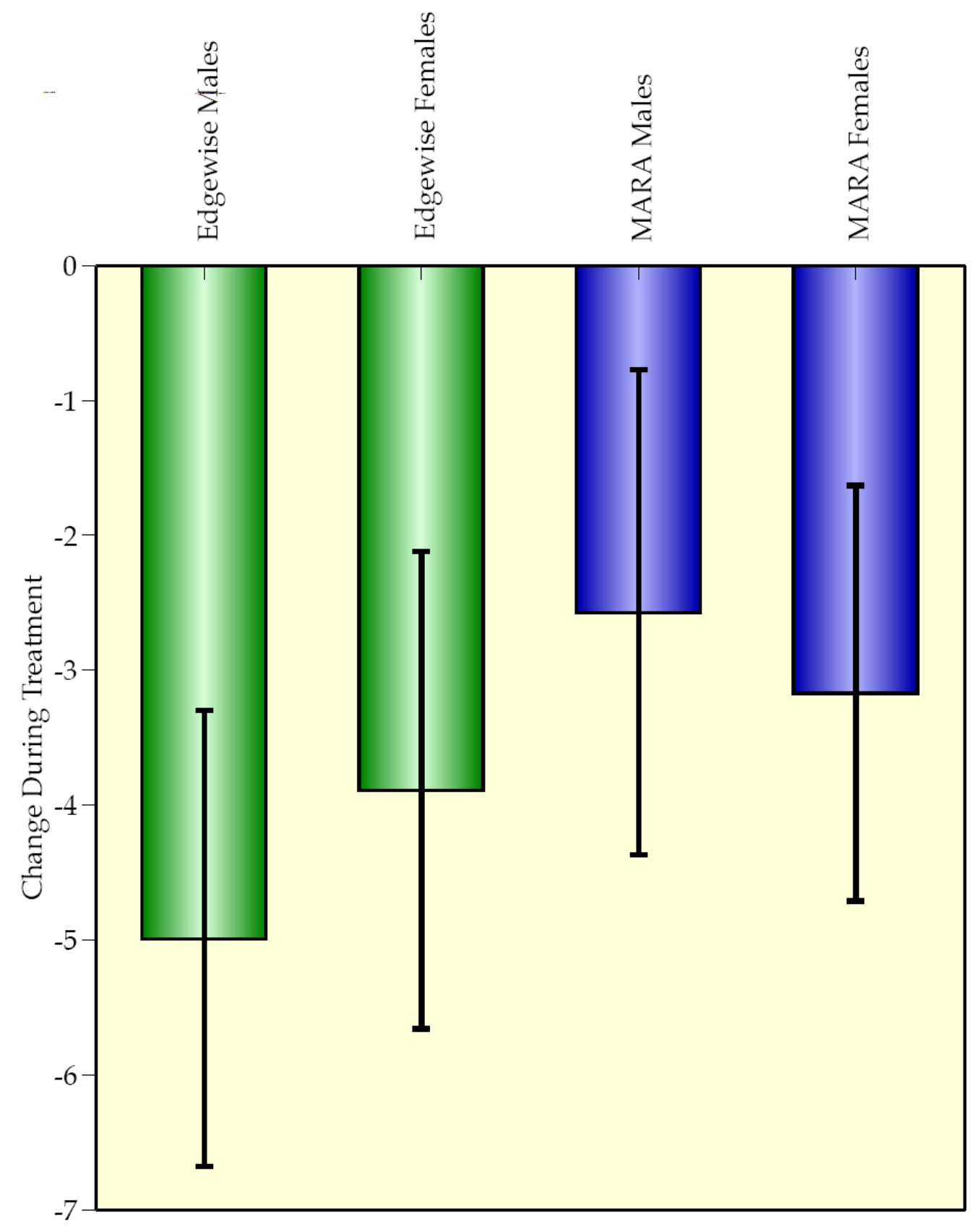

Figure E-22. Mean in-treatment changes in overjet, by treatment and by sex. Error bars are \pm one standard error of the mean. 


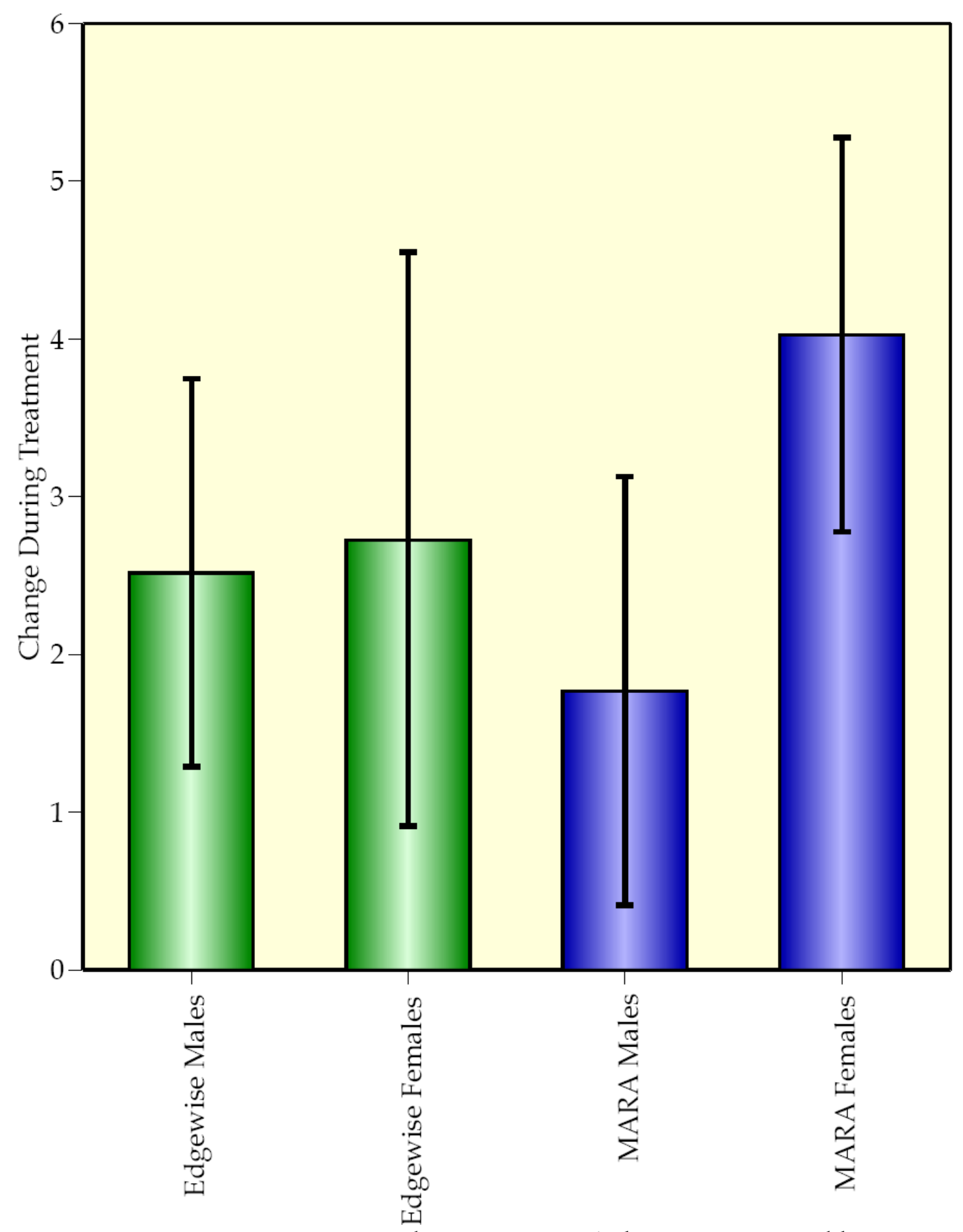

Figure E-23. Mean in-treatment changes in IMPA, by treatment and by sex. Error bars are \pm one standard error of the mean. 


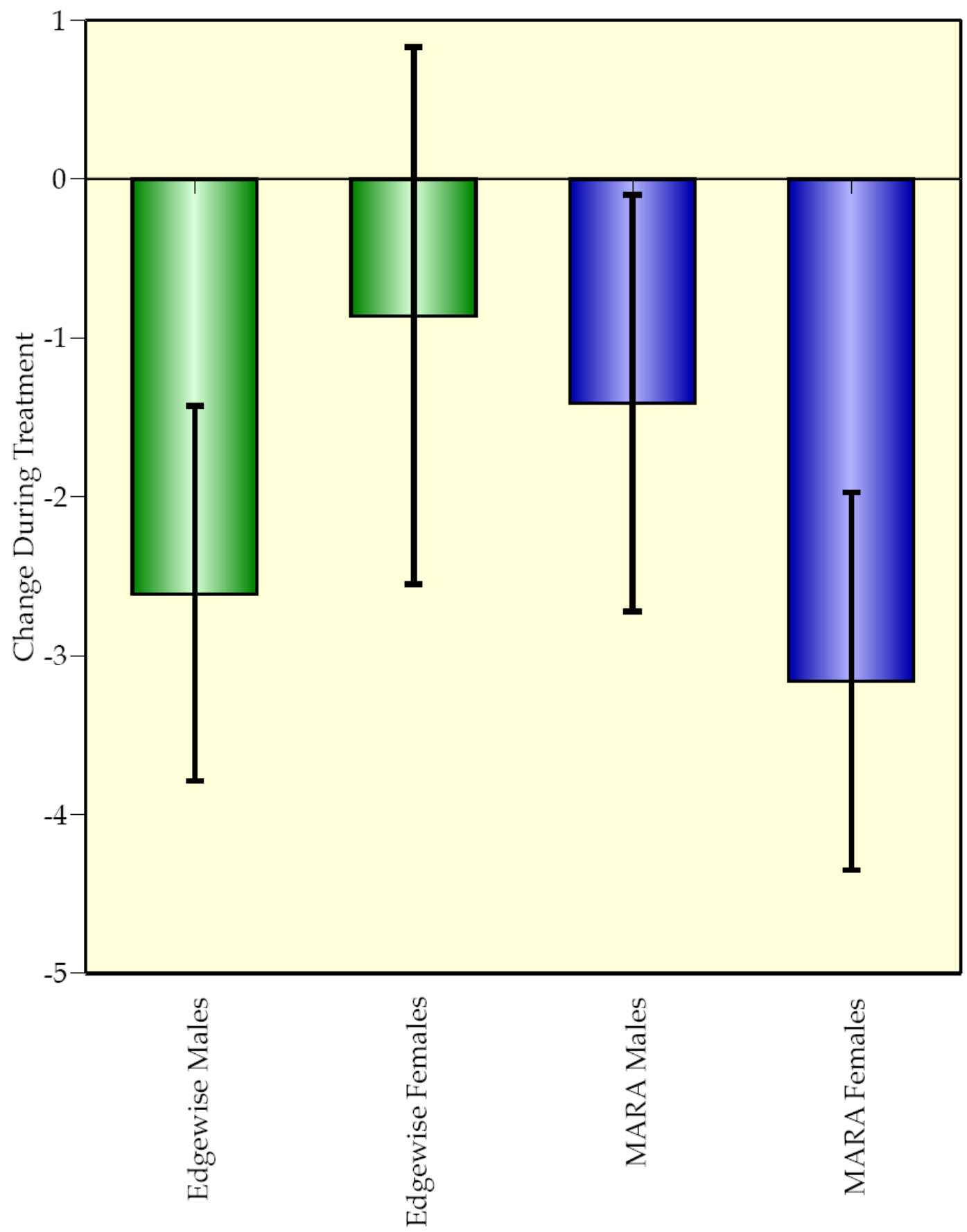

Figure E-24. Mean in-treatment changes in FMIA, by treatment and by sex. Error bars are \pm one standard error of the mean. 


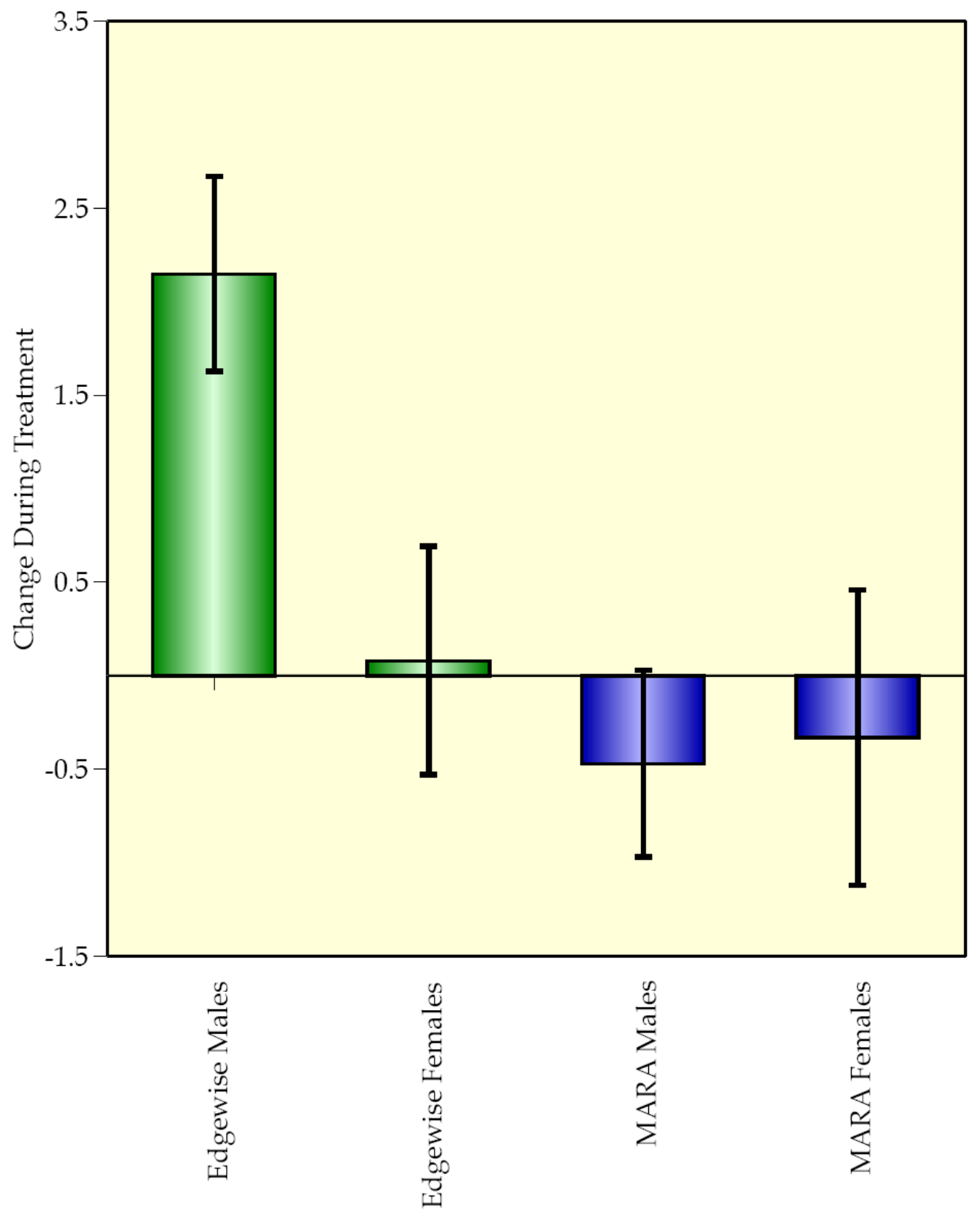

Figure E-25. Mean in-treatment changes in OP to FH, by treatment and by sex. Error bars are \pm one standard error of the mean. 


\section{APPENDIX F. CEPHALOMETRIC TRACINGS}

Prints of the cephalometric tracings (superimpositions) of the subjects used in the present study. For the MARA sample, the black lines are at pretreatment and the red lines are at end of treatment. For the Edgewise sample, the black lines are at pre-treatment and the red lines are at end of treatment. Each superimposition is followed by its matched counterpart superimposition. All tracings are printed at true size (i.e., 1:1 with the original radiograph). 


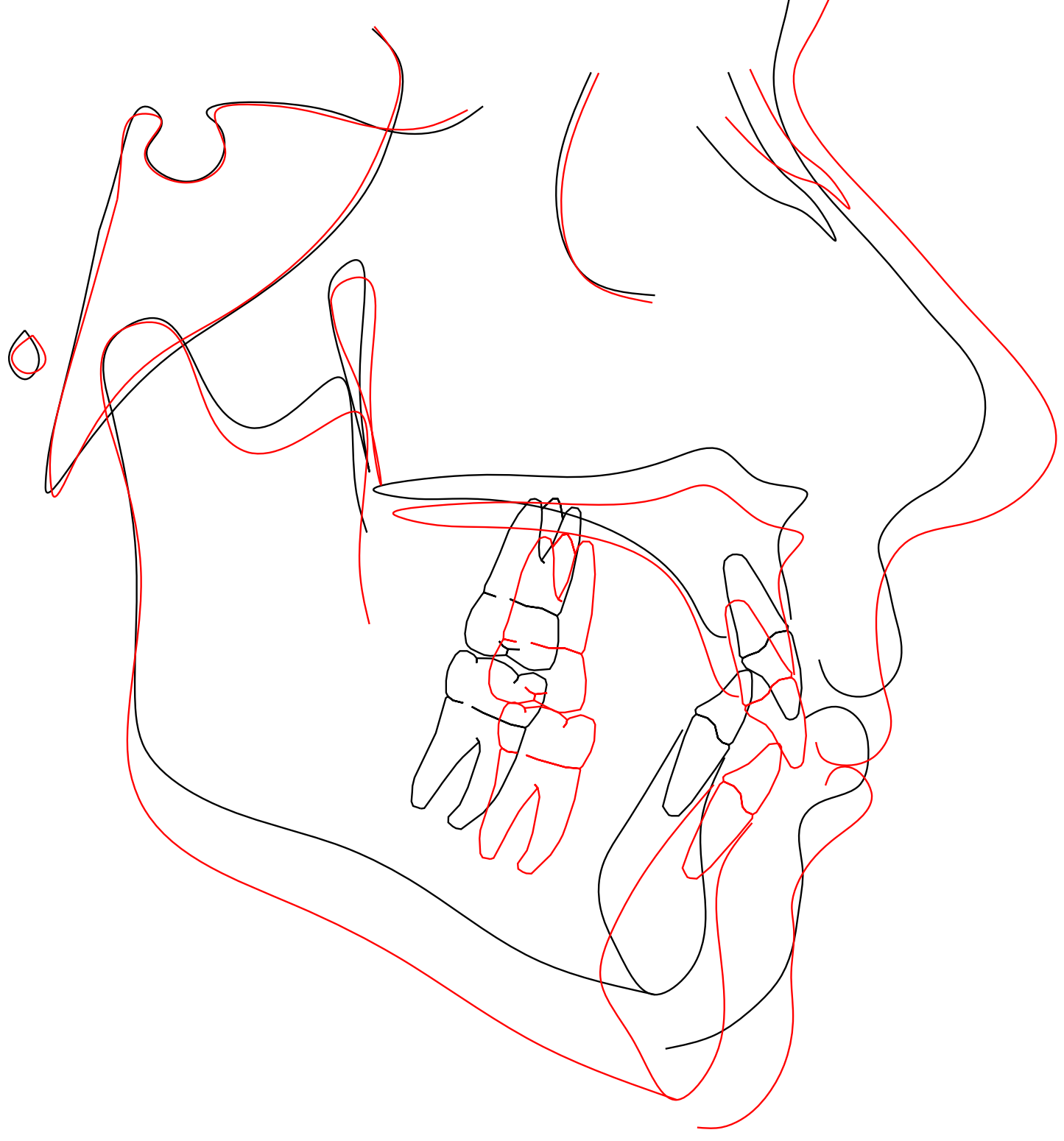

Figure F-1. Print of cephalometric tracing superimposition subject 01: (tracing is 1:1 with original radiograph). 


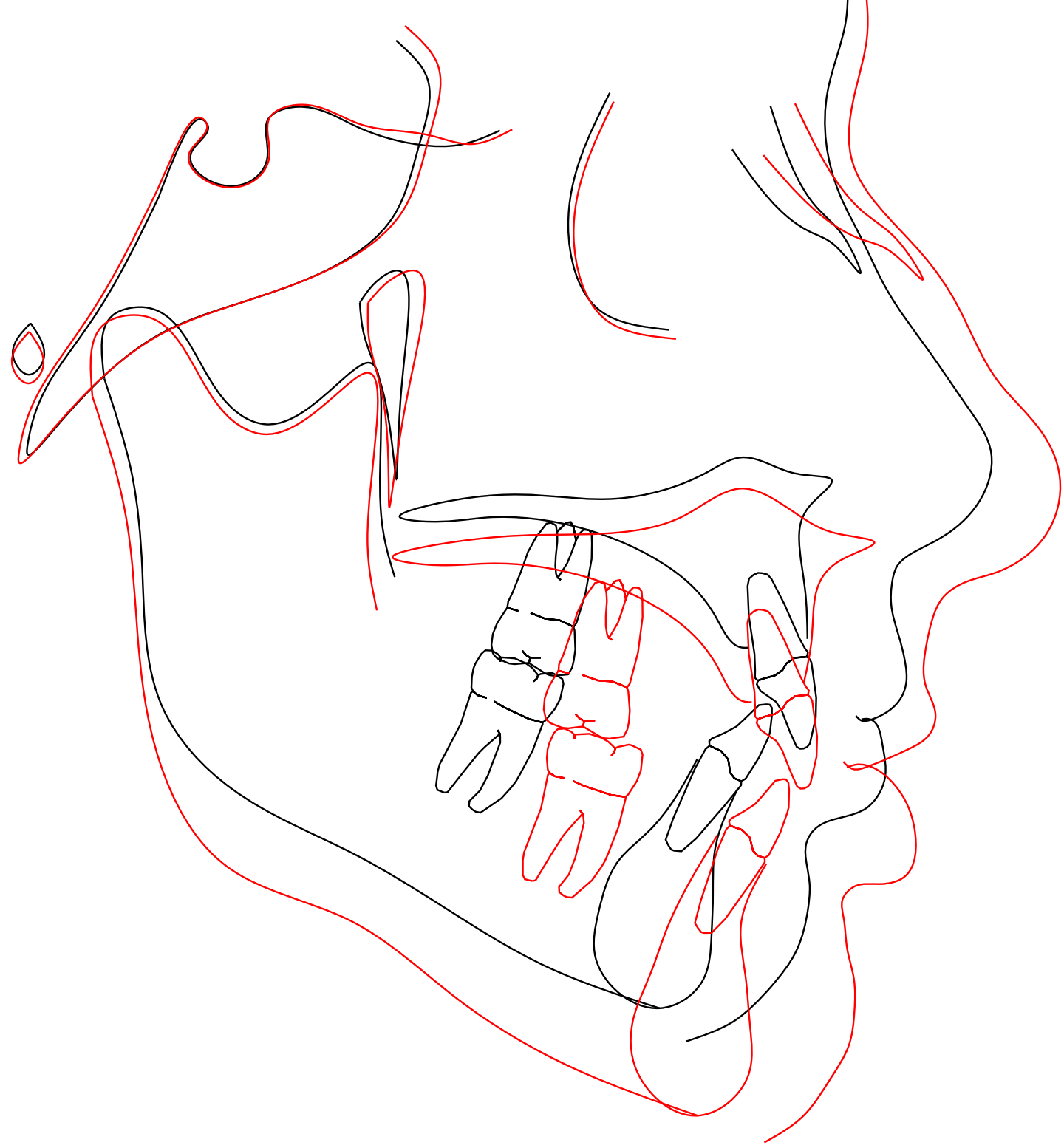

Figure F-2. Print of cephalometric tracing superimposition subject 02: (tracing is 1:1 with original radiograph). 


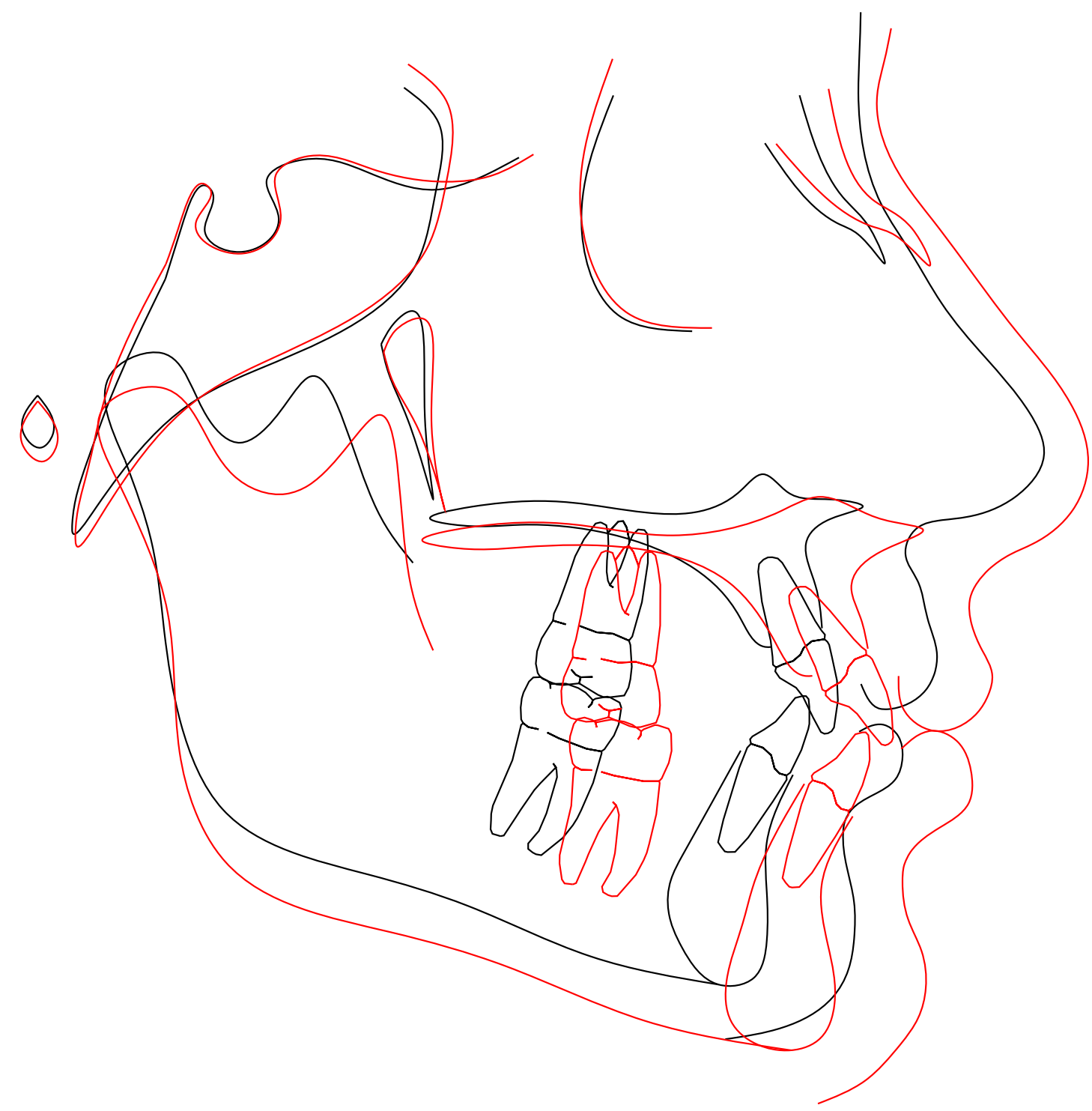

Figure F-3. Print of cephalometric tracing superimposition subject 03: (tracing is 1:1 with original radiograph). 


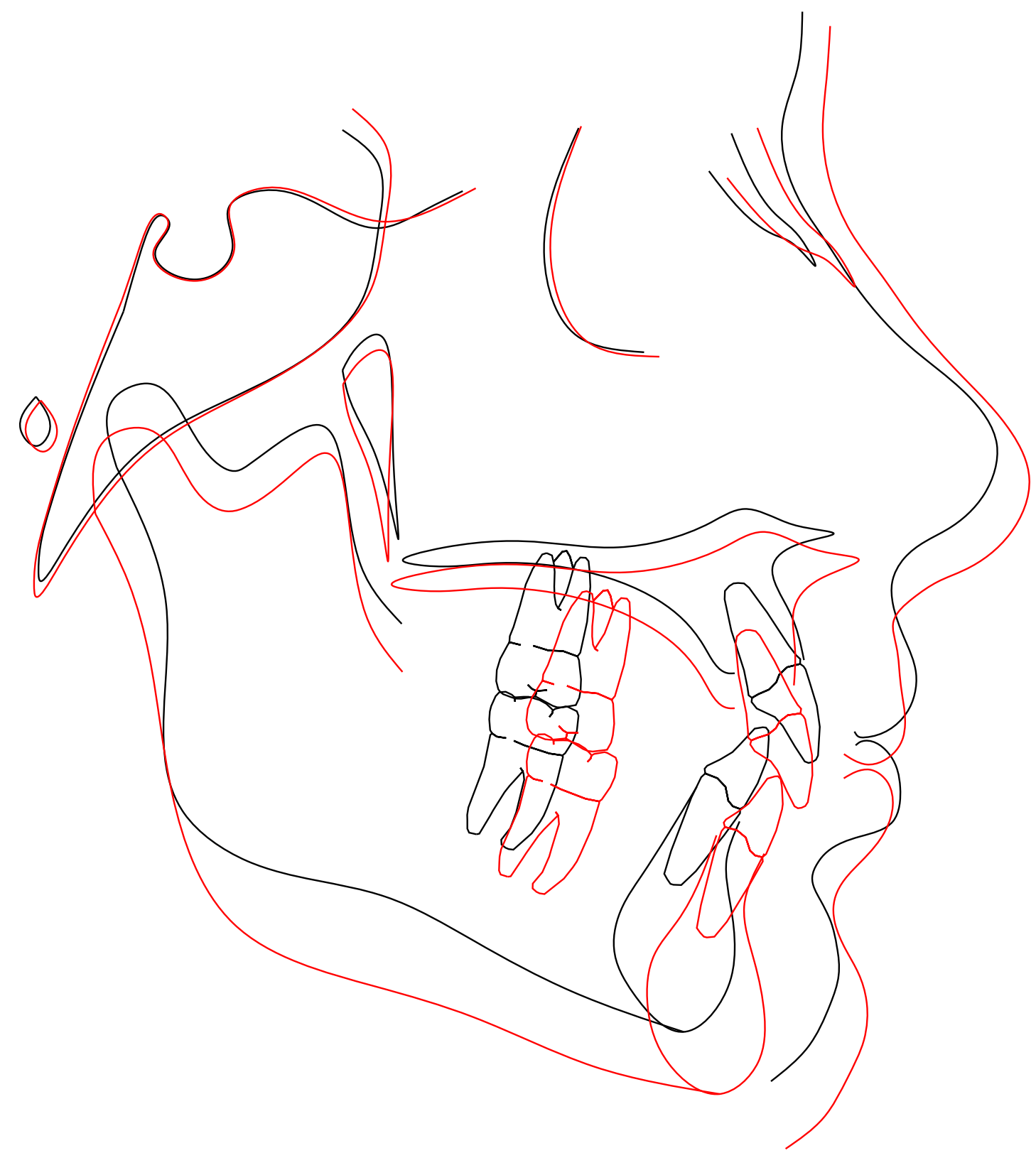

Figure F-4. Print of cephalometric tracing superimposition subject 04: (tracing is 1:1 with original radiograph). 


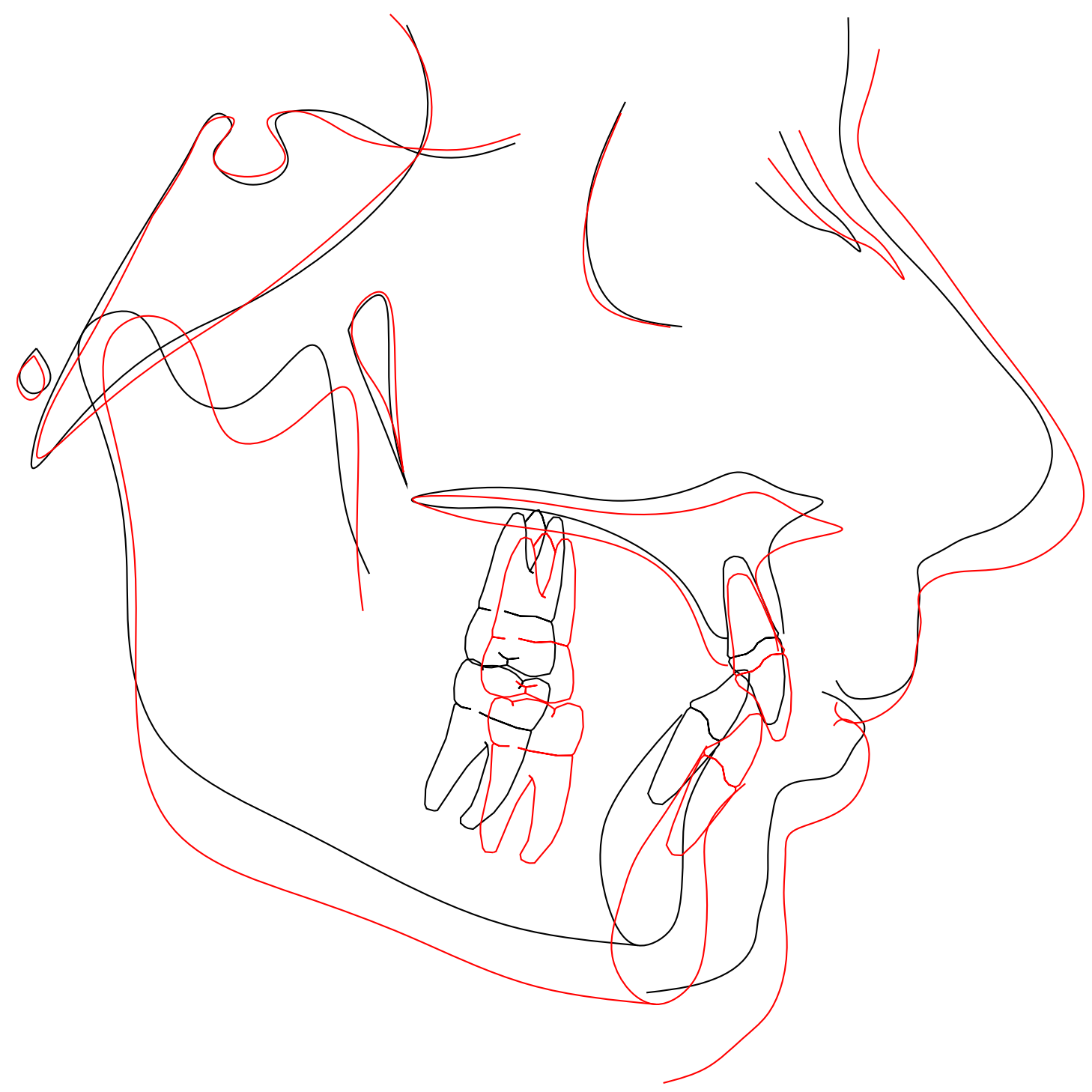

Figure F-5. Print of cephalometric tracing superimposition subject 05: (tracing is 1:1 with original radiograph). 


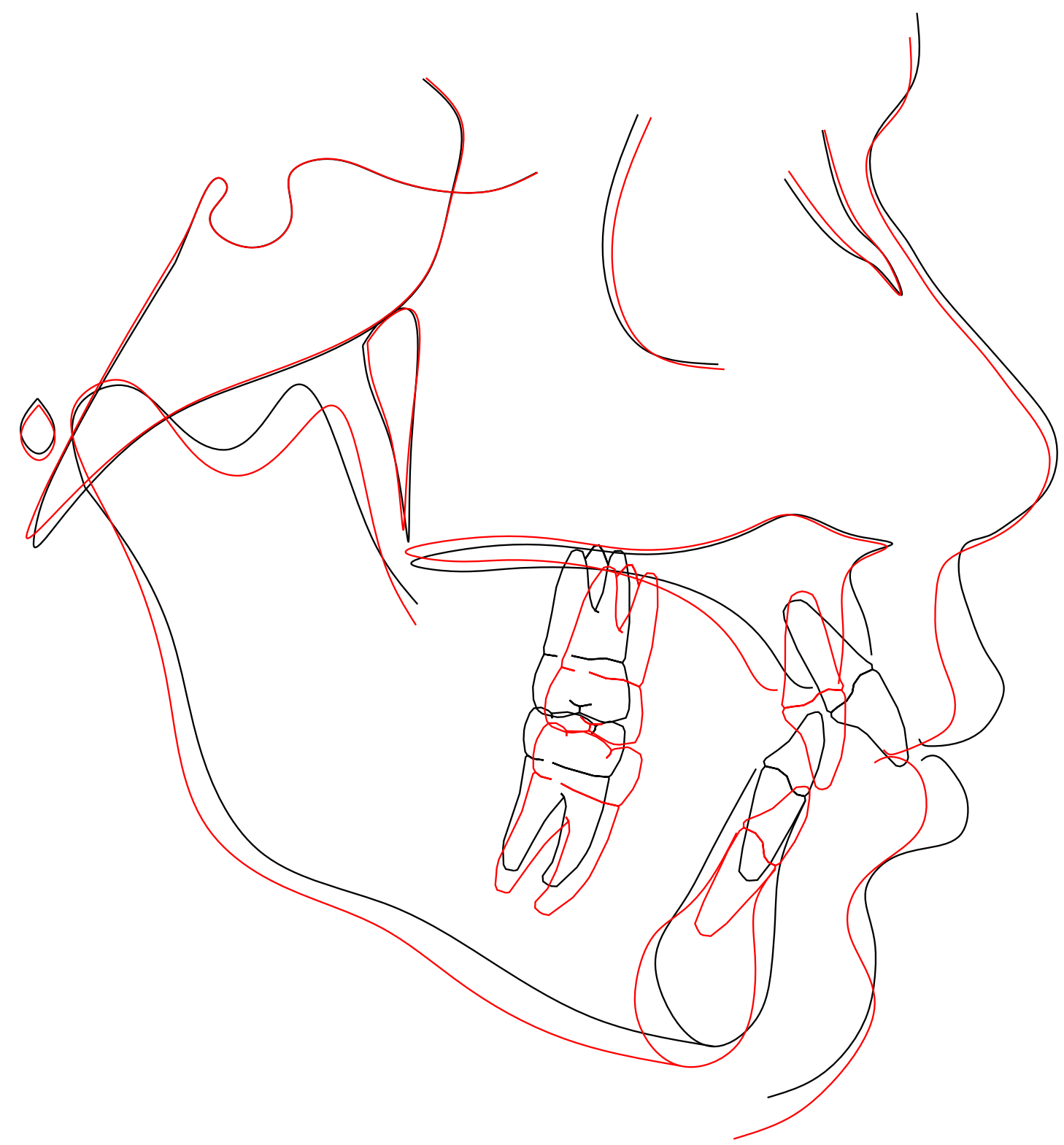

Figure F-6. Print of cephalometric tracing superimposition subject 06: (tracing is 1:1 with original radiograph). 


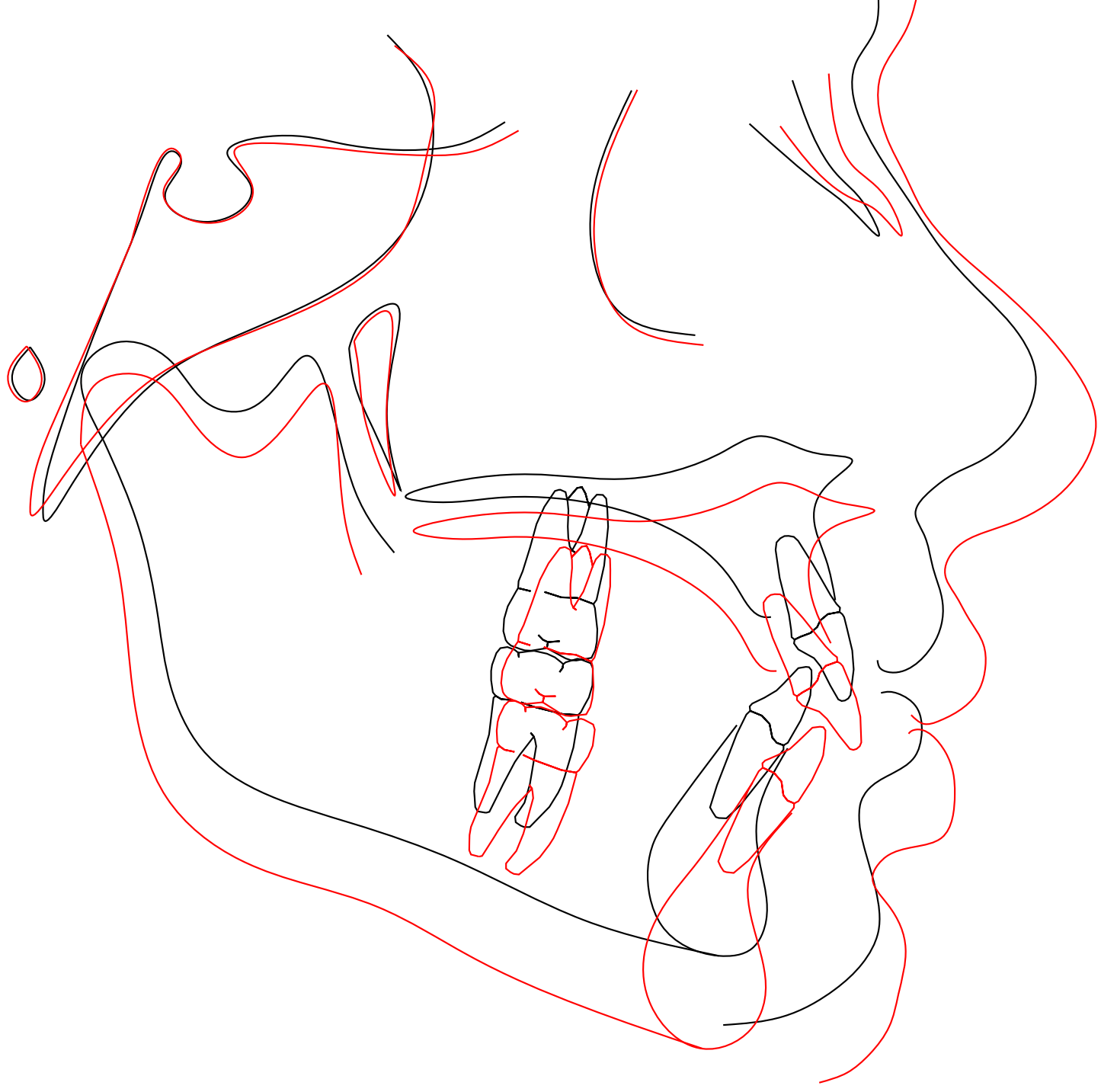

Figure F-7. Print of cephalometric tracing superimposition subject 07: (tracing is 1:1 with original radiograph). 


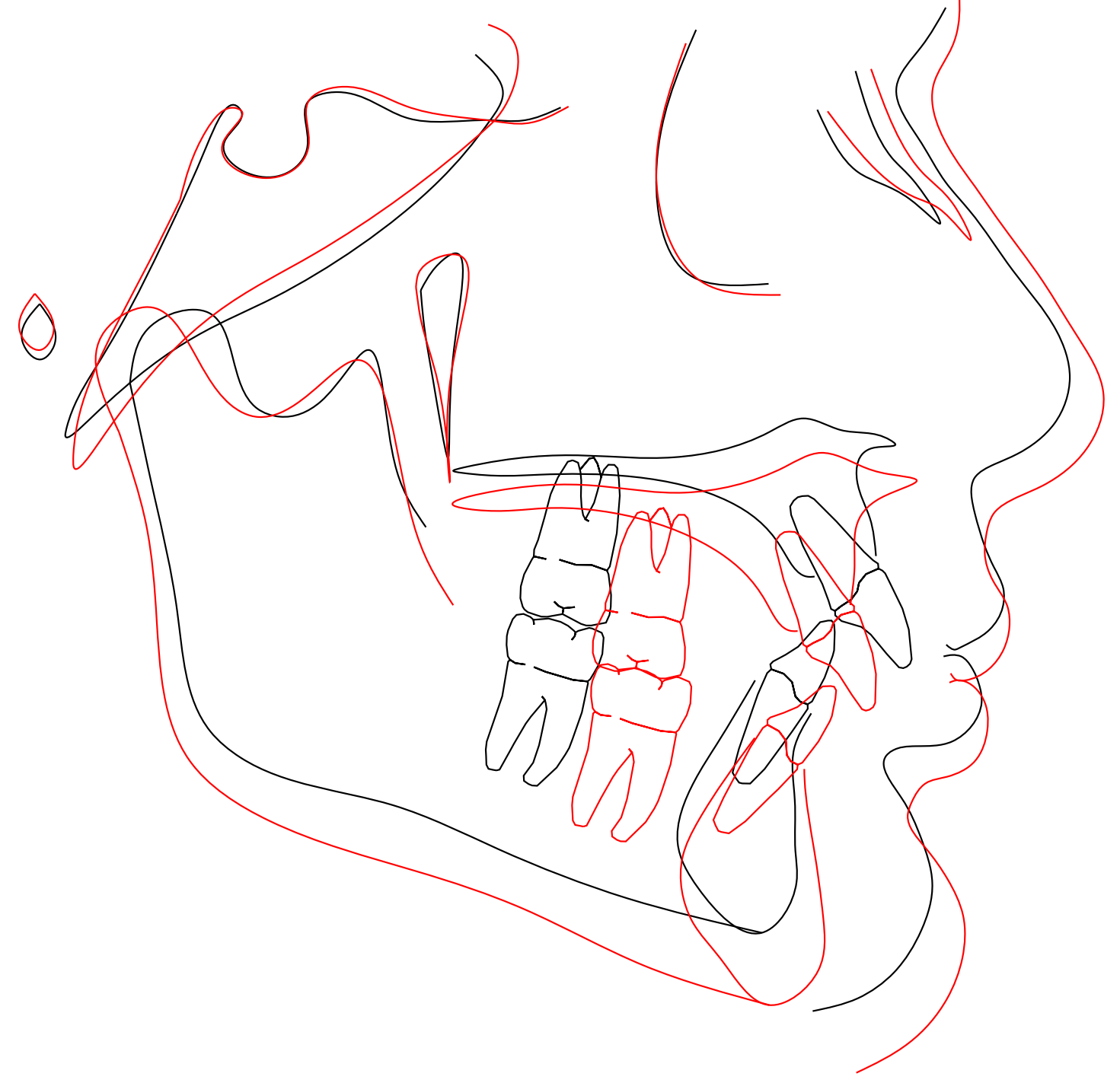

Figure F-8. Print of cephalometric tracing superimposition subject 08: (tracing is 1:1 with original radiograph). 


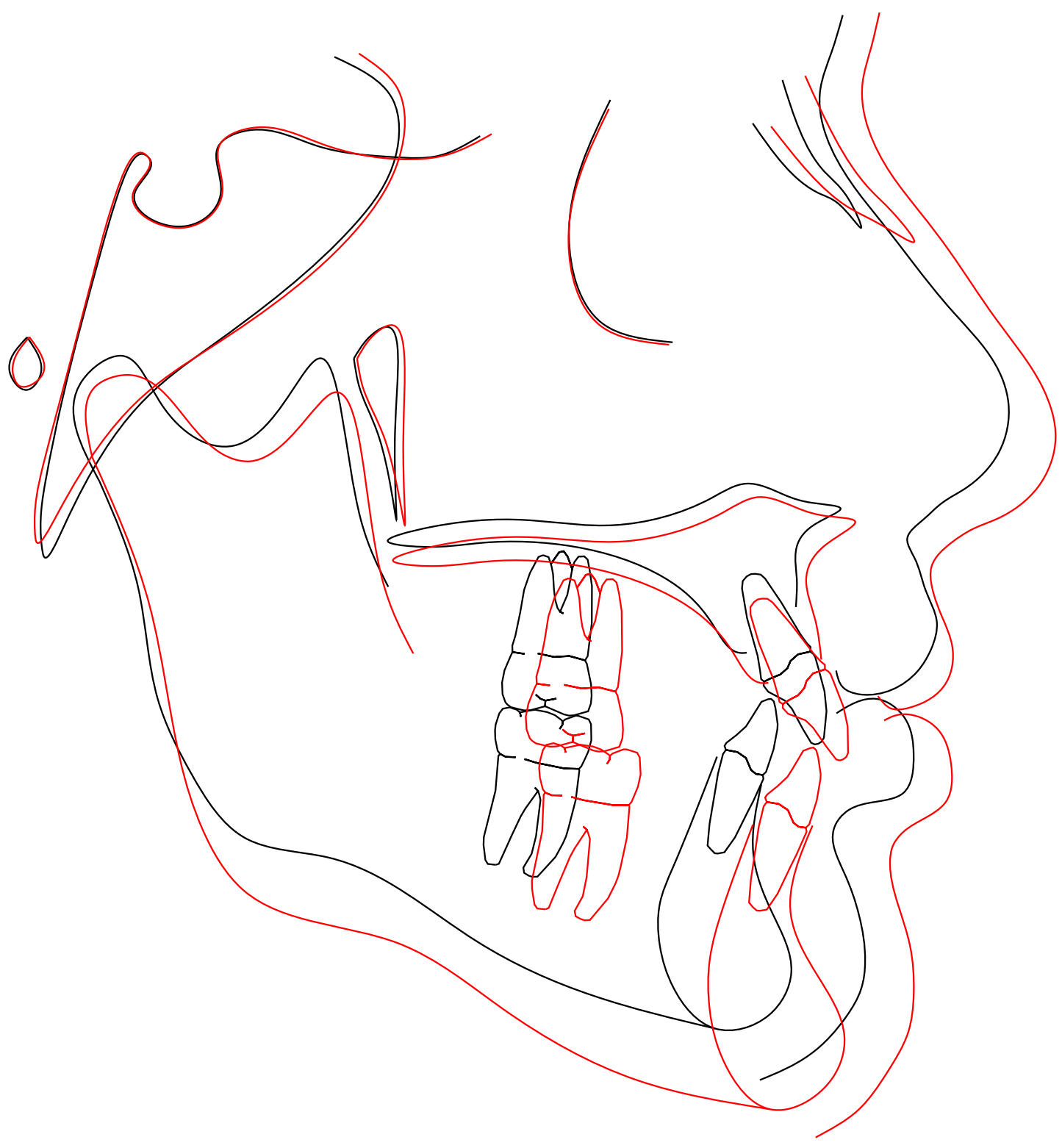

Figure F-9. Print of cephalometric tracing superimposition subject 09: (tracing is 1:1 with original radiograph). 


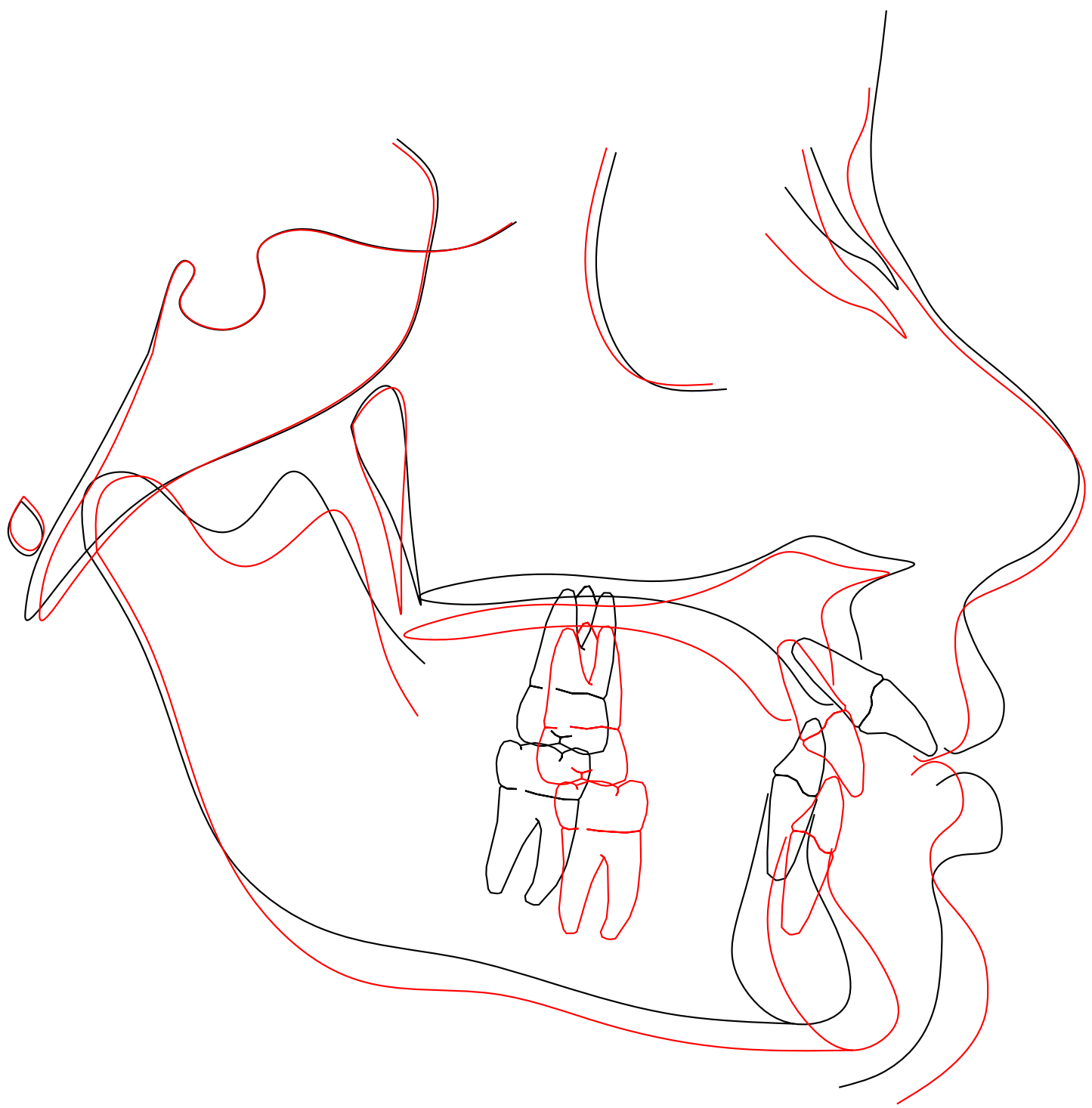

Figure F-10. Print of cephalometric tracing superimposition subject 10: (tracing is 1:1 with original radiograph). 


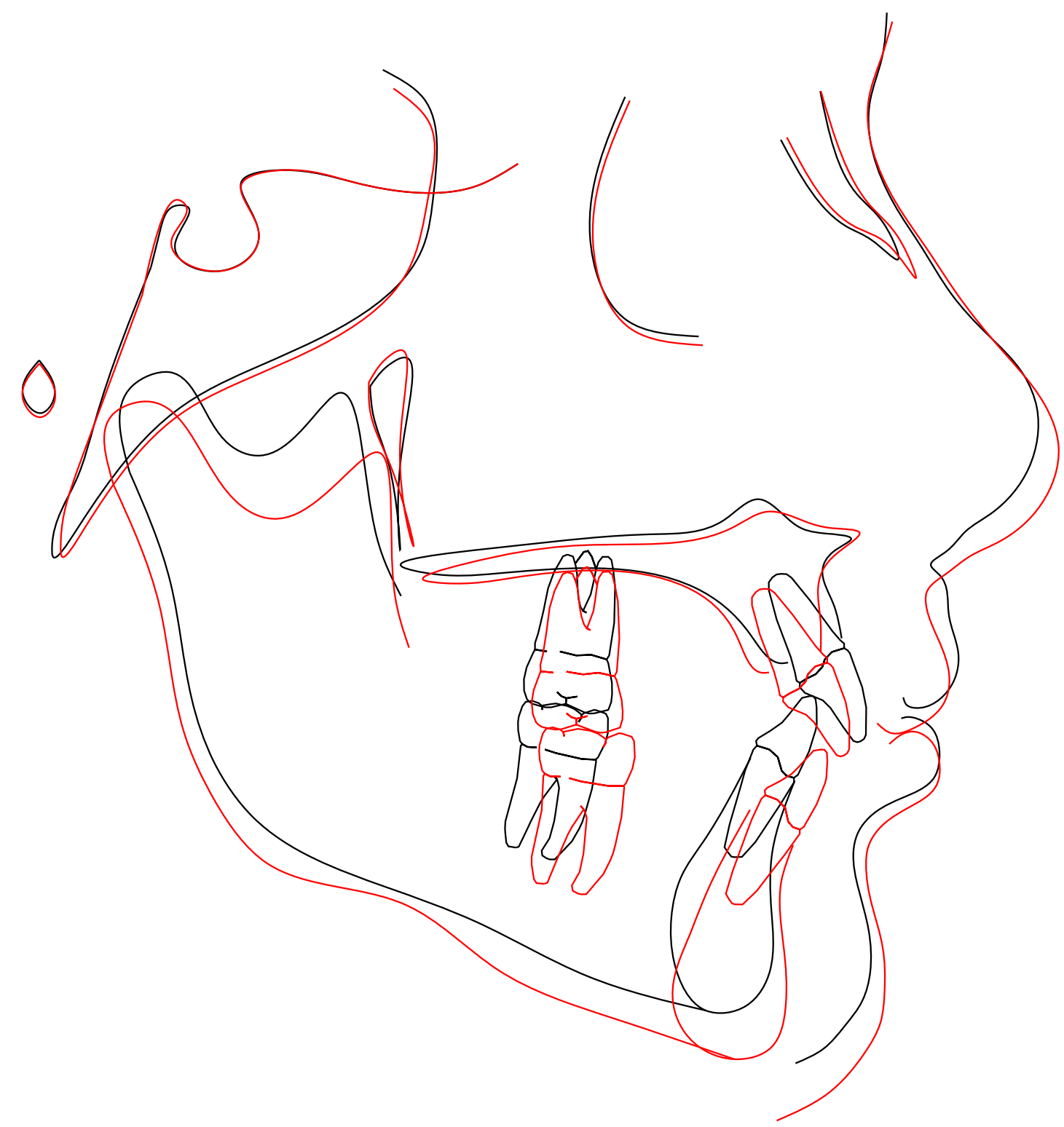

Figure F-11. Print of cephalometric tracing superimposition subject 11: (tracing is 1:1 with original radiograph). 


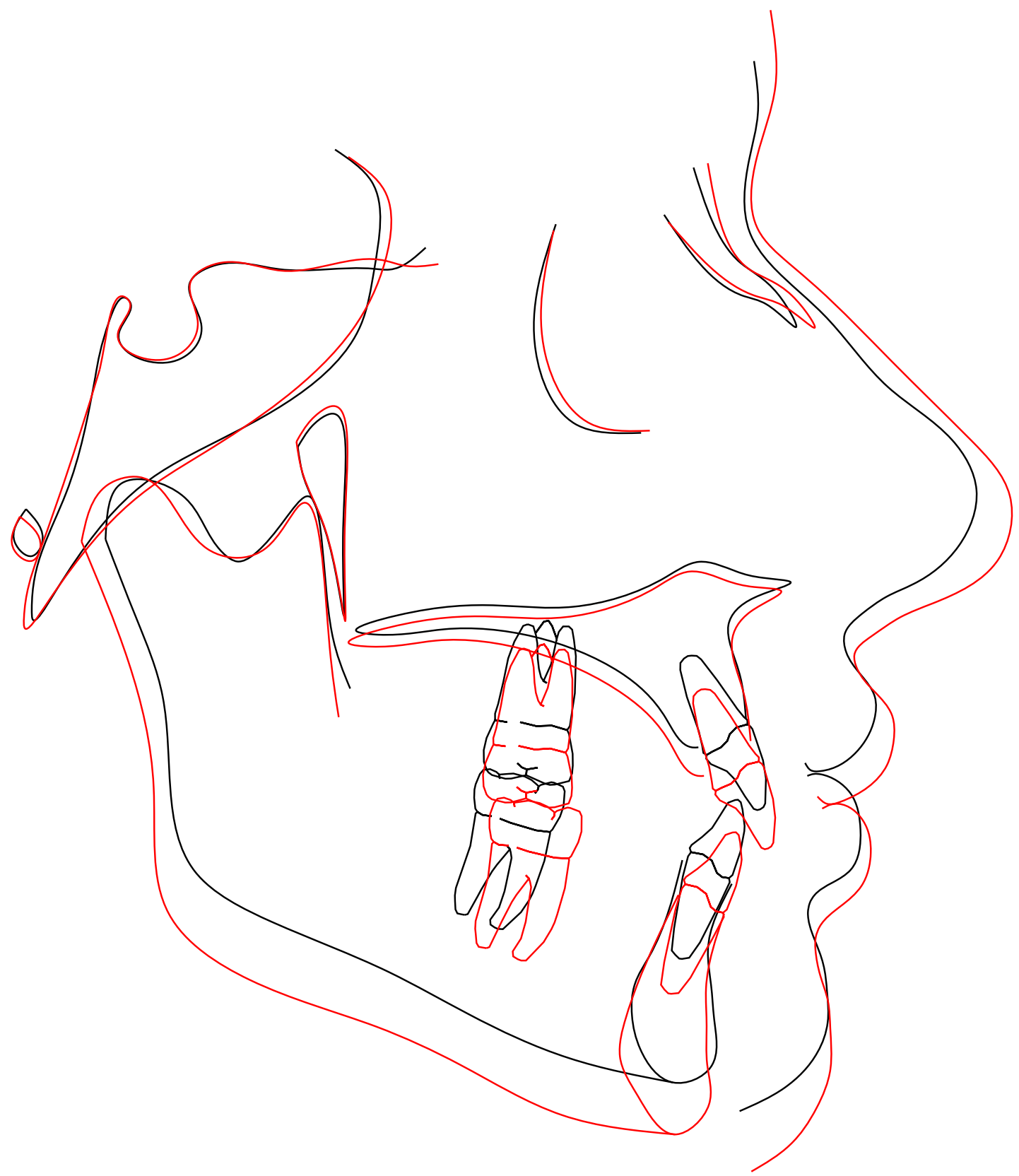

Figure F-12. Print of cephalometric tracing superimposition subject 12: (tracing is 1:1 with original radiograph). 


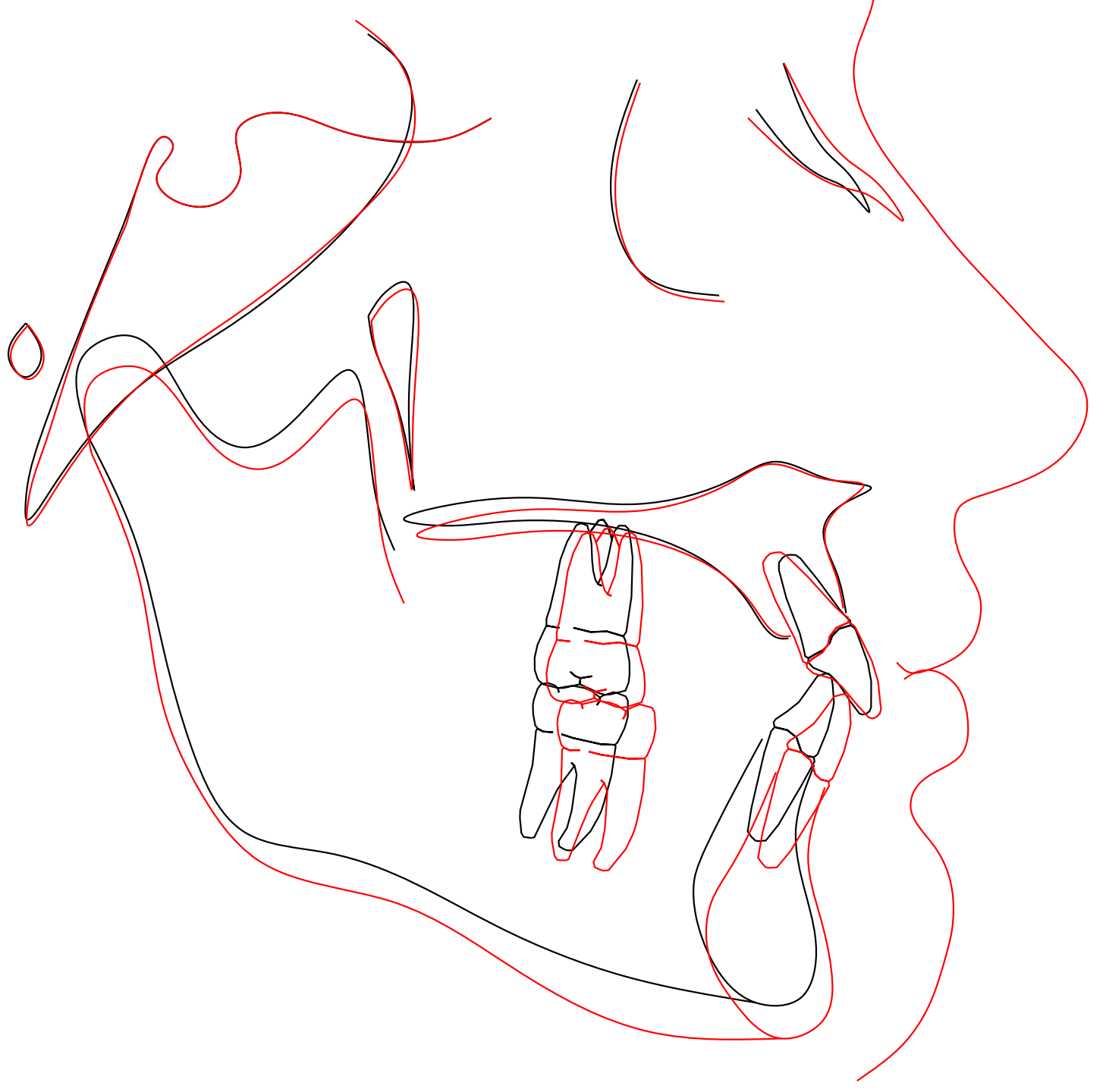

Figure F-13. Print of cephalometric tracing superimposition subject 13: (tracing is 1:1 with original radiograph). 


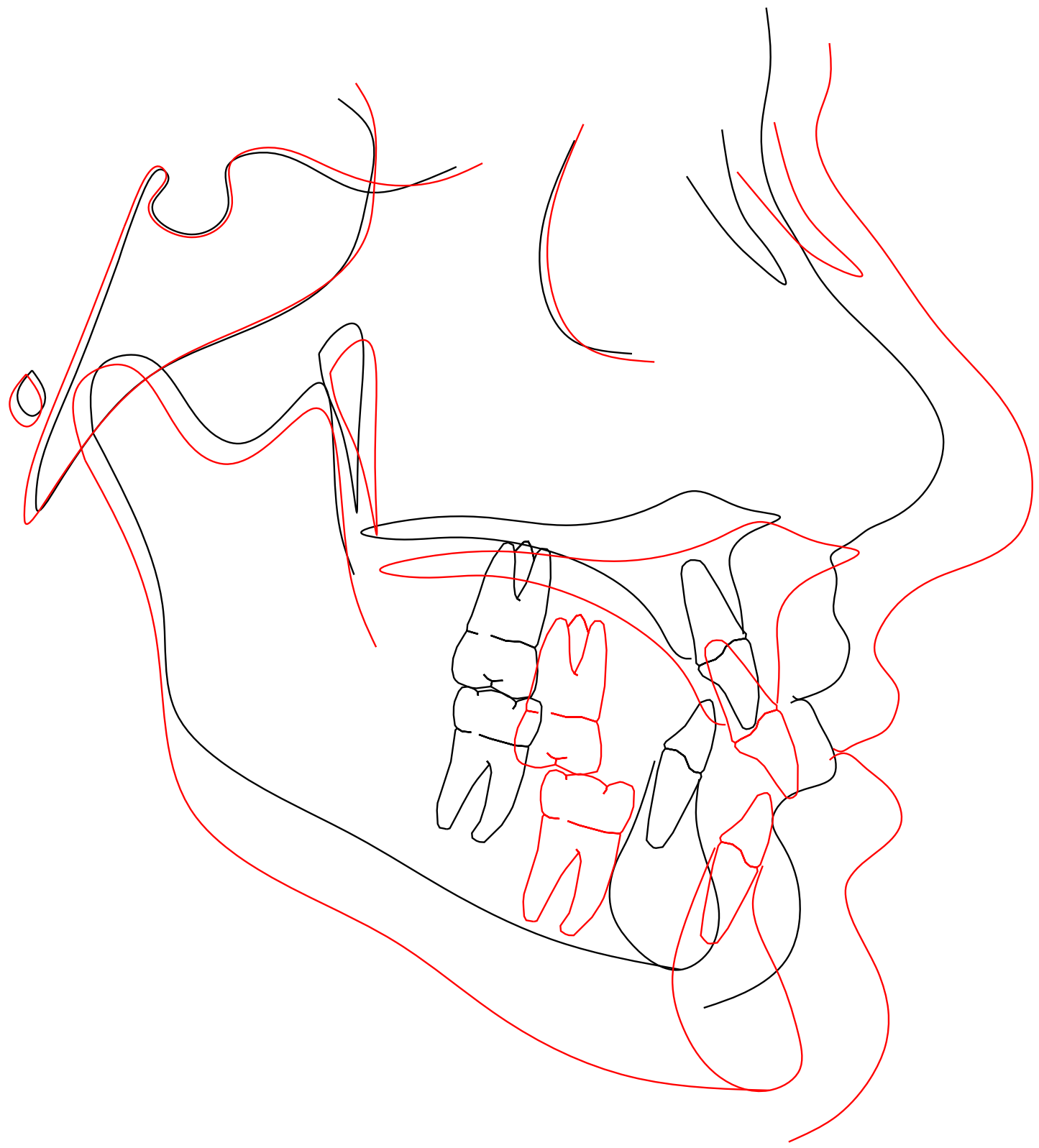

Figure F-14. Print of cephalometric tracing superimposition subject 14: (tracing is 1:1 with original radiograph). 


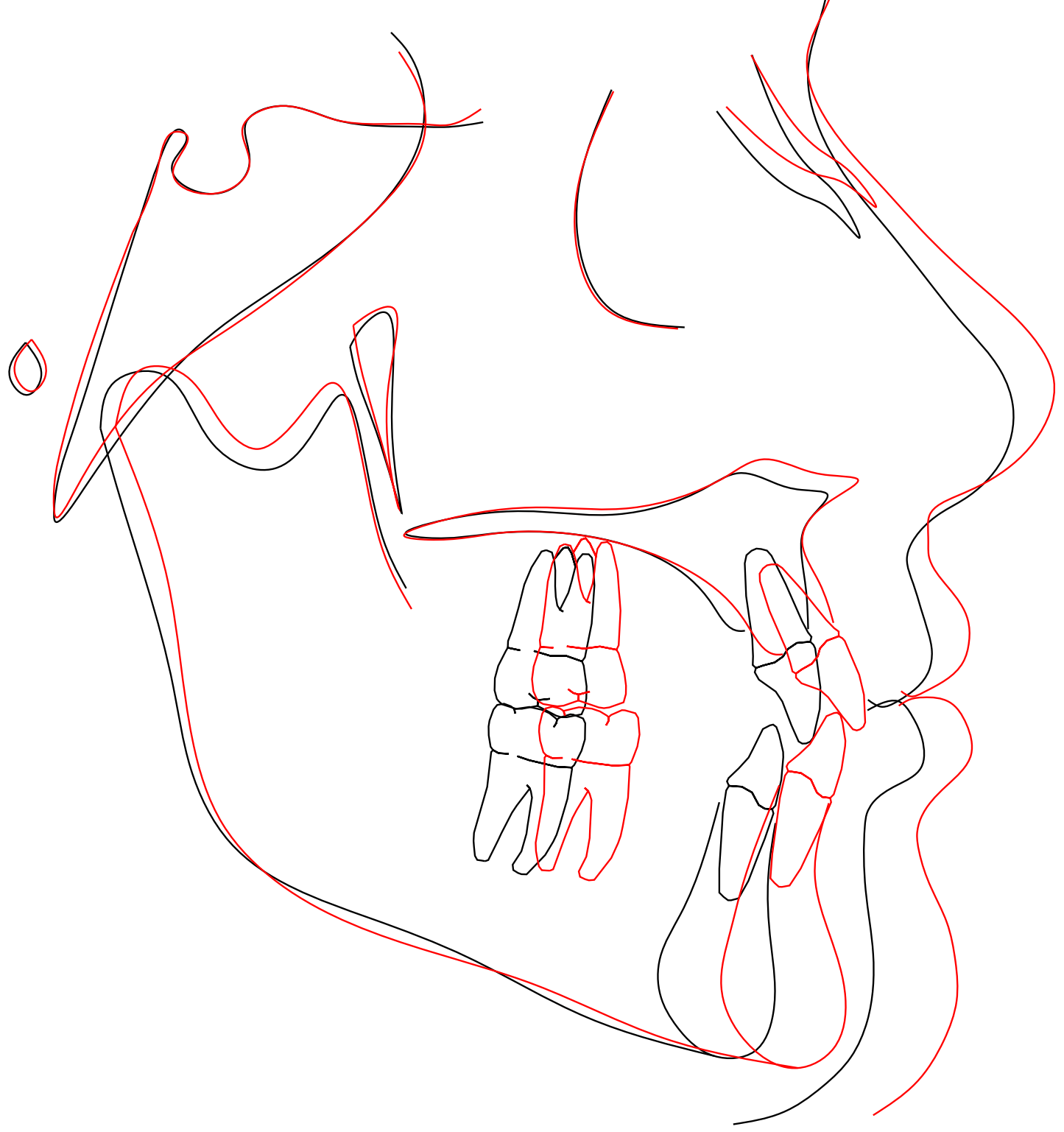

Figure F-15. Print of cephalometric tracing superimposition subject 15: (tracing is 1:1 with original radiograph). 


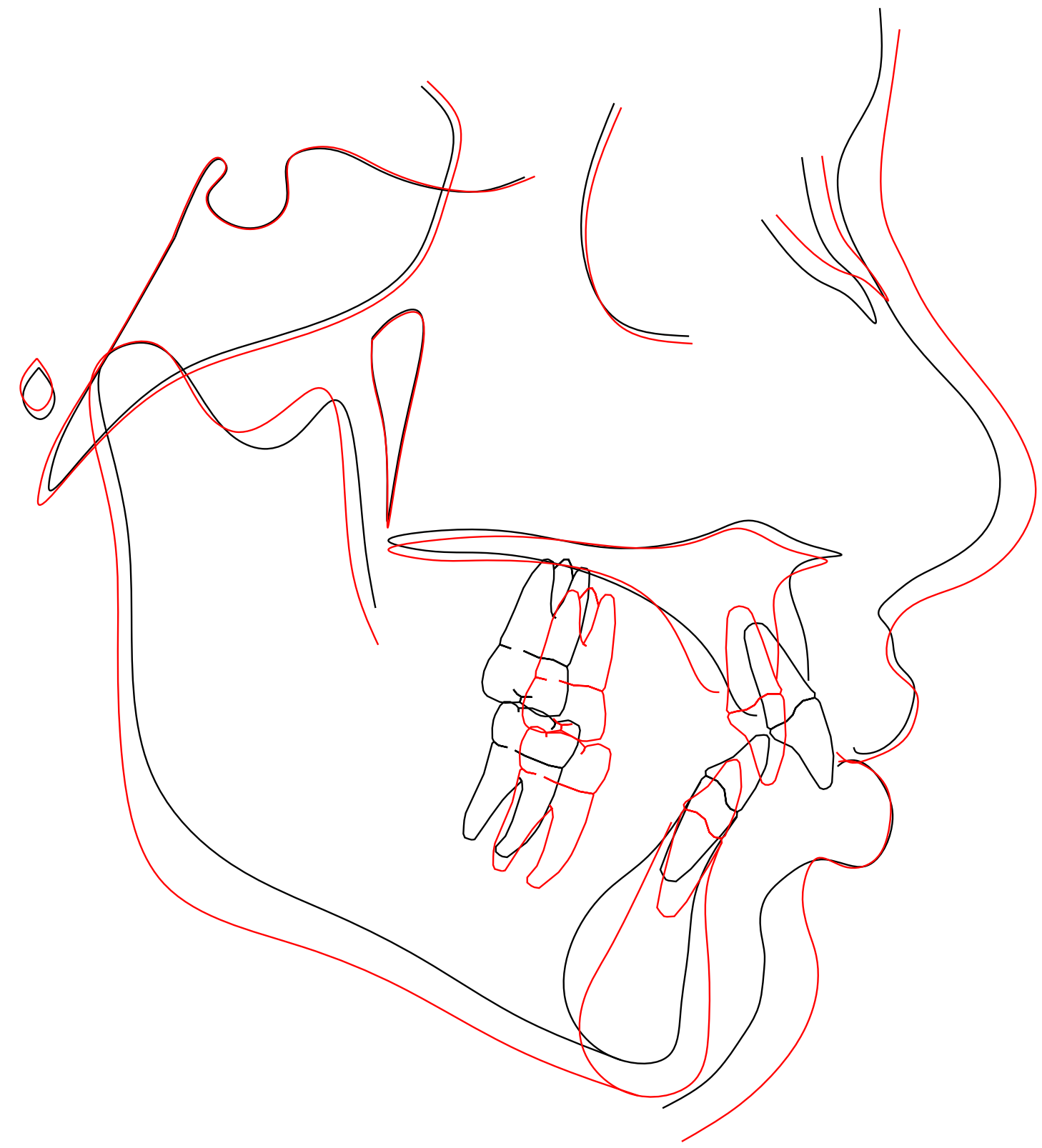

Figure F-16. Print of cephalometric tracing superimposition subject 16: (tracing is 1:1 with original radiograph). 


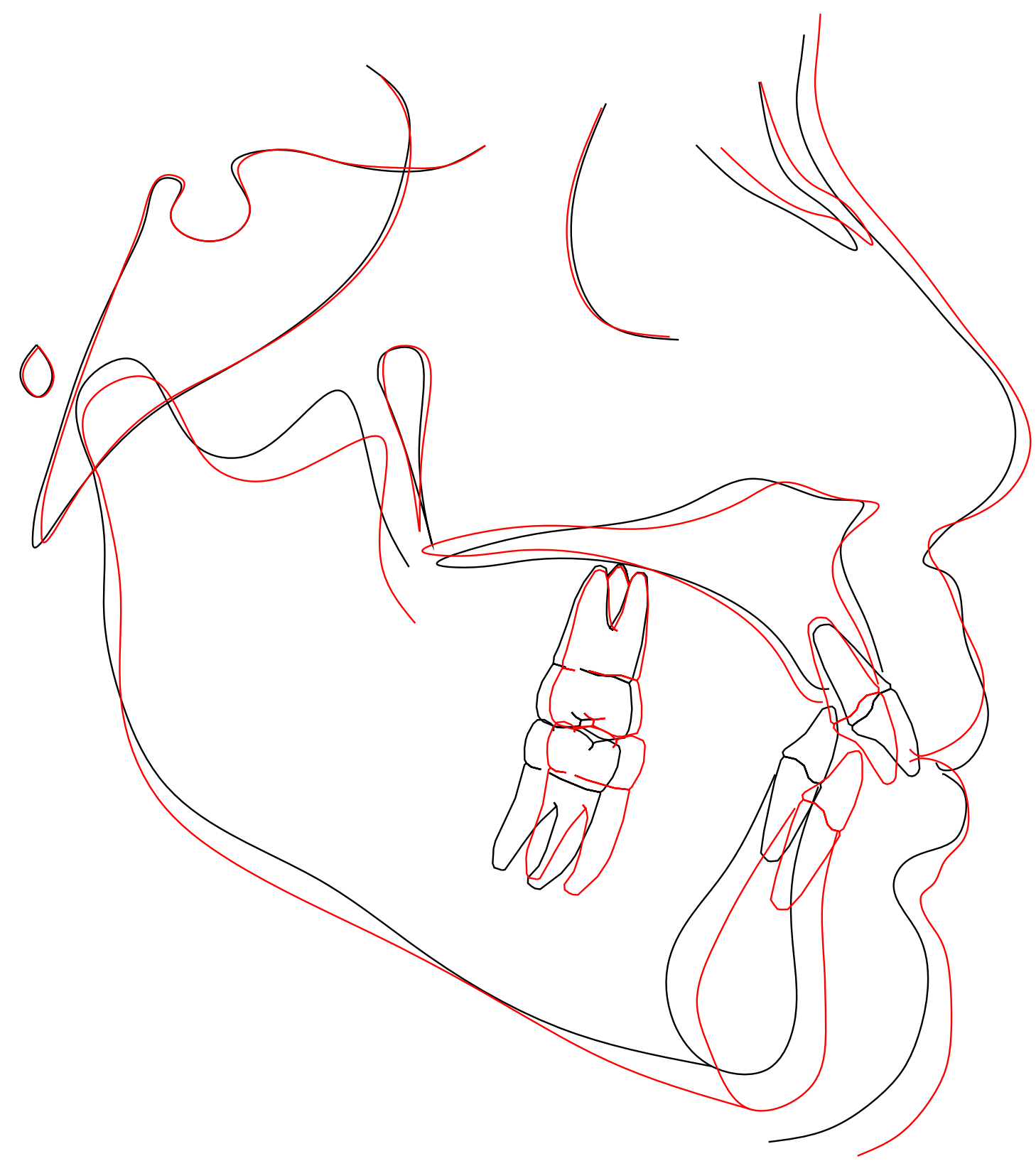

Figure F-17. Print of cephalometric tracing superimposition subject 17: (tracing is 1:1 with original radiograph). 


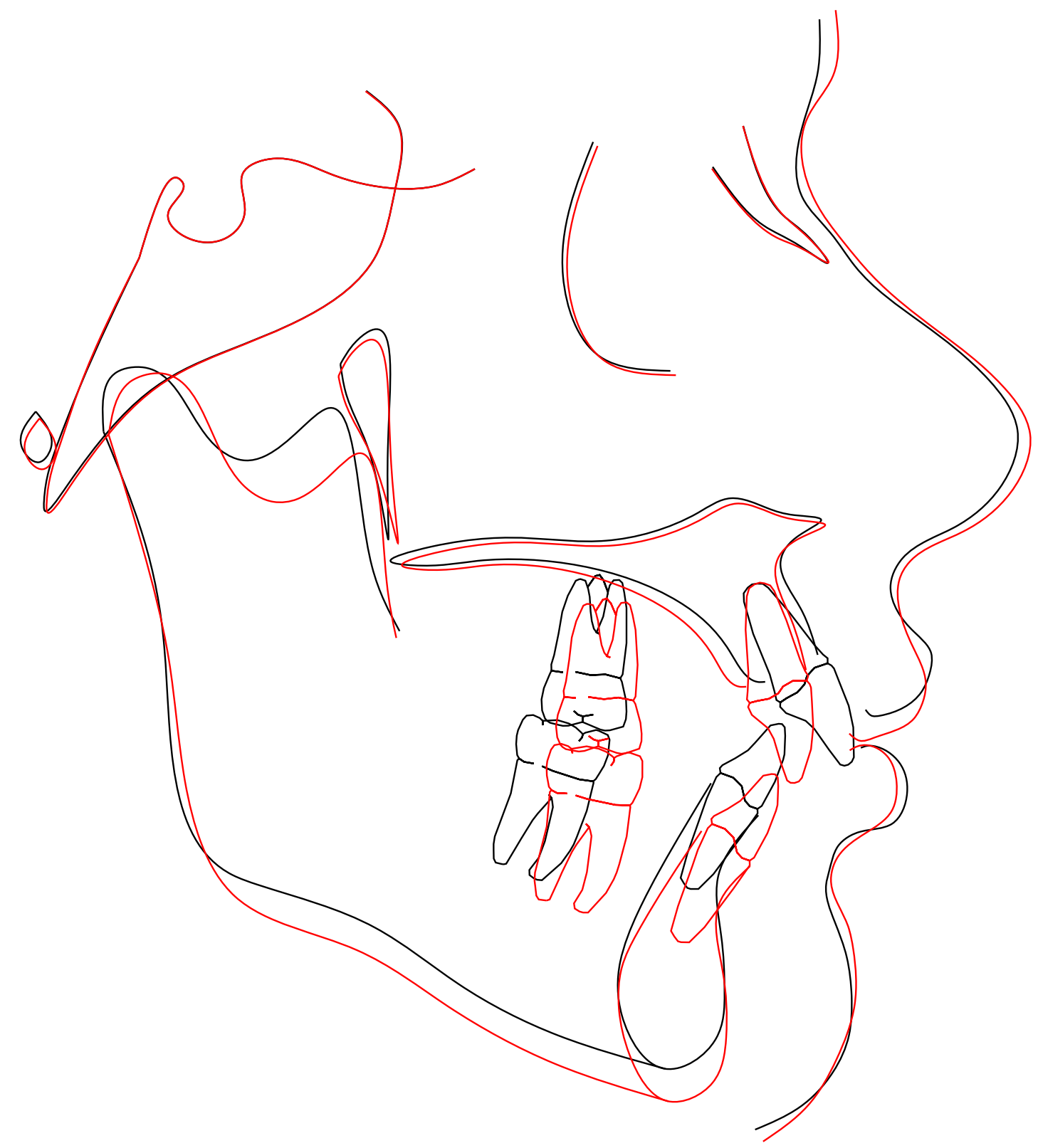

Figure F-18. Print of cephalometric tracing superimposition subject 18: (tracing is 1:1 with original radiograph). 


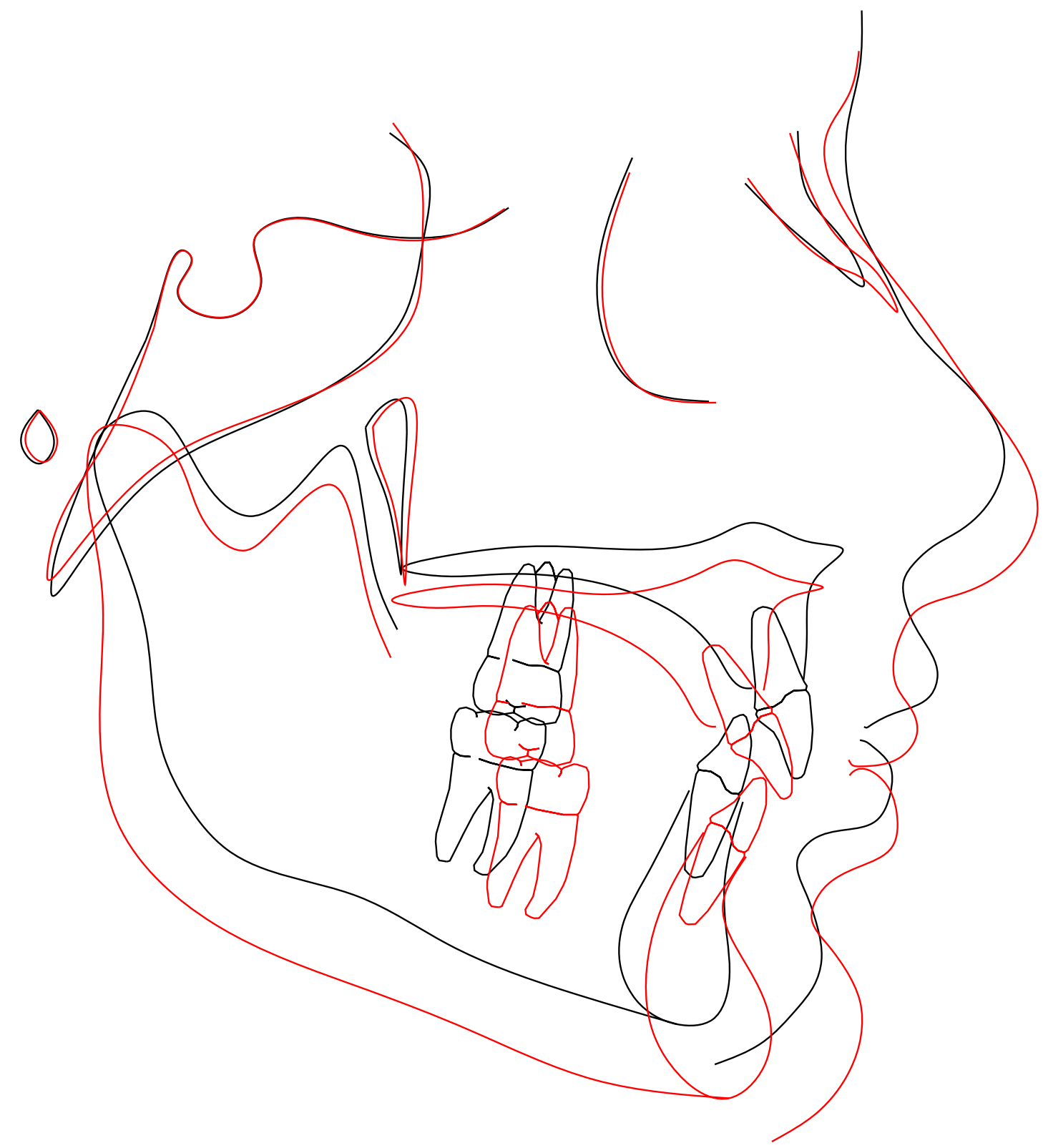

Figure F-19. Print of cephalometric tracing superimposition subject 19: (tracing is 1:1 with original radiograph). 


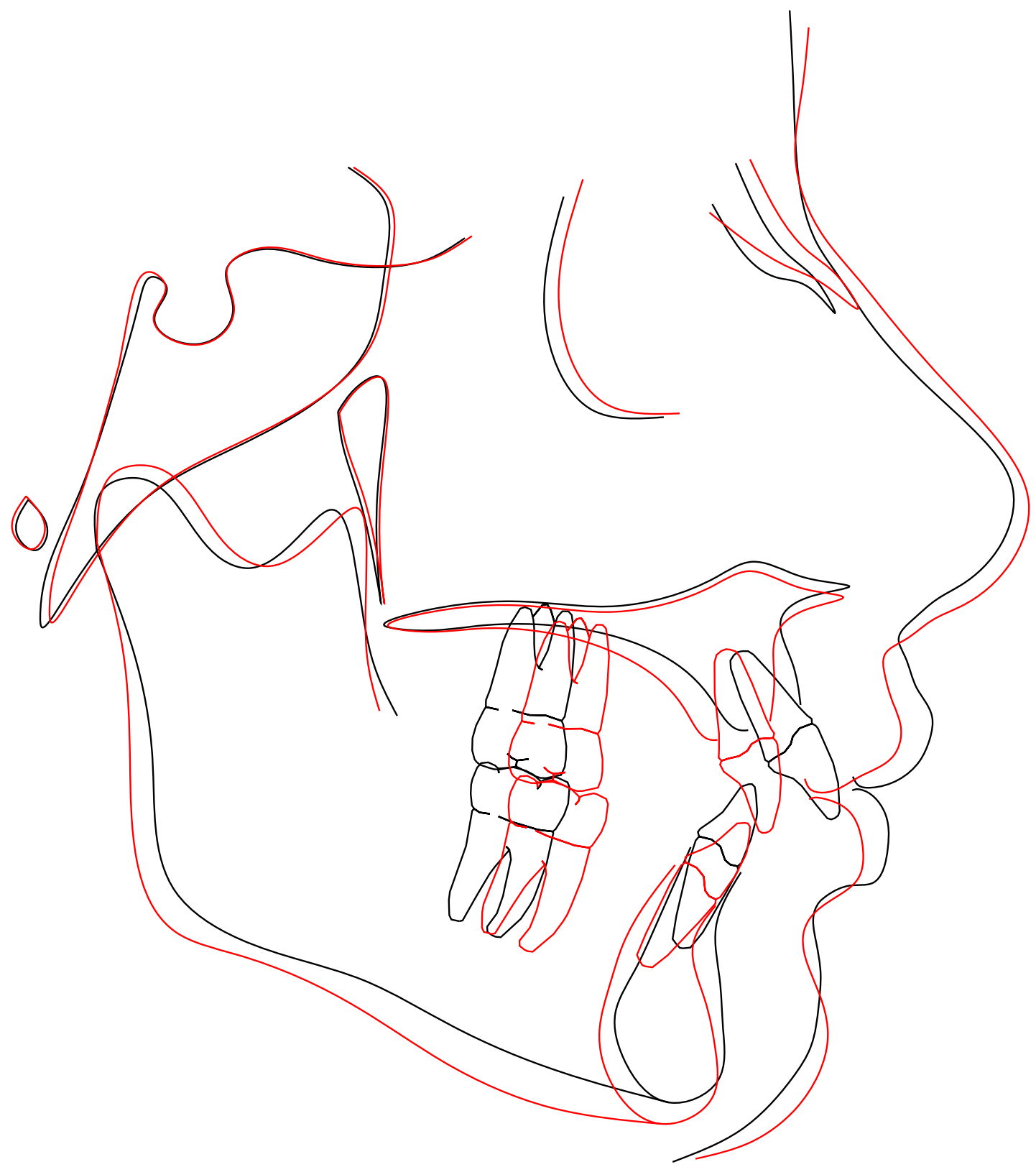

Figure F-20. Print of cephalometric tracing superimposition subject 20: (tracing is 1:1 with original radiograph). 


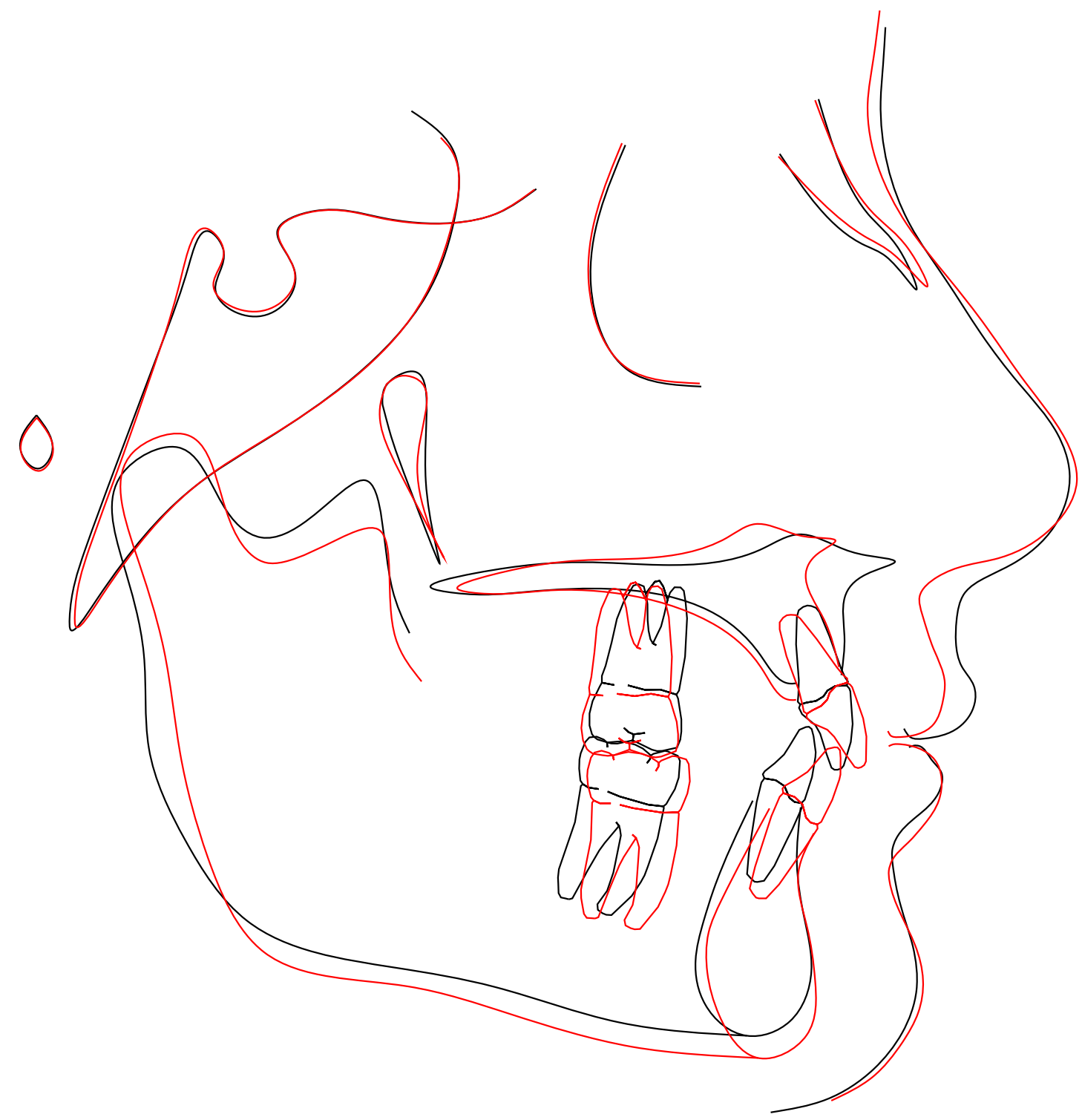

Figure F-21. Print of cephalometric tracing superimposition subject 21: (tracing is 1:1 with original radiograph). 


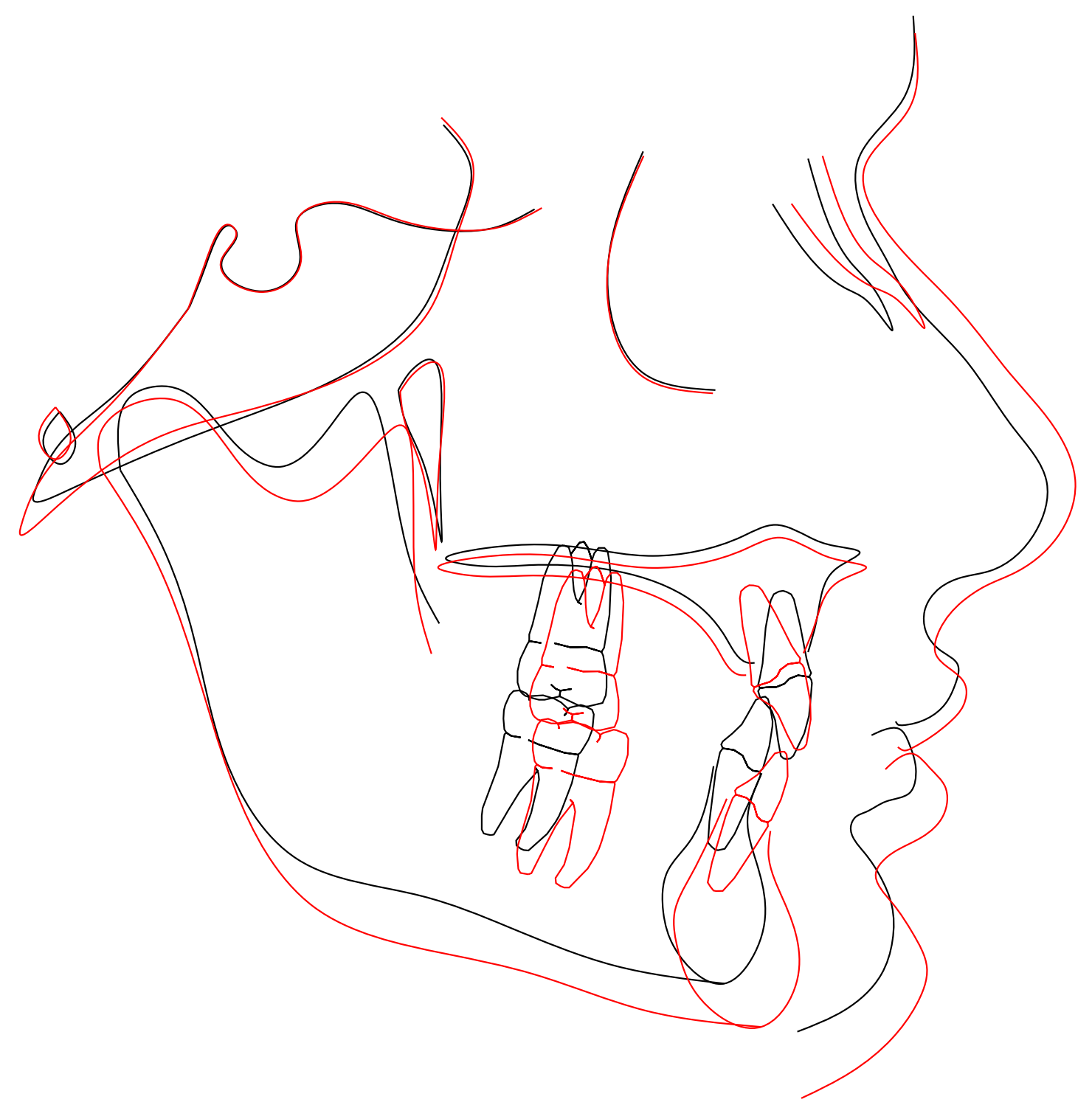

Figure F-22. Print of cephalometric tracing superimposition subject 22: (tracing is 1:1 with original radiograph). 


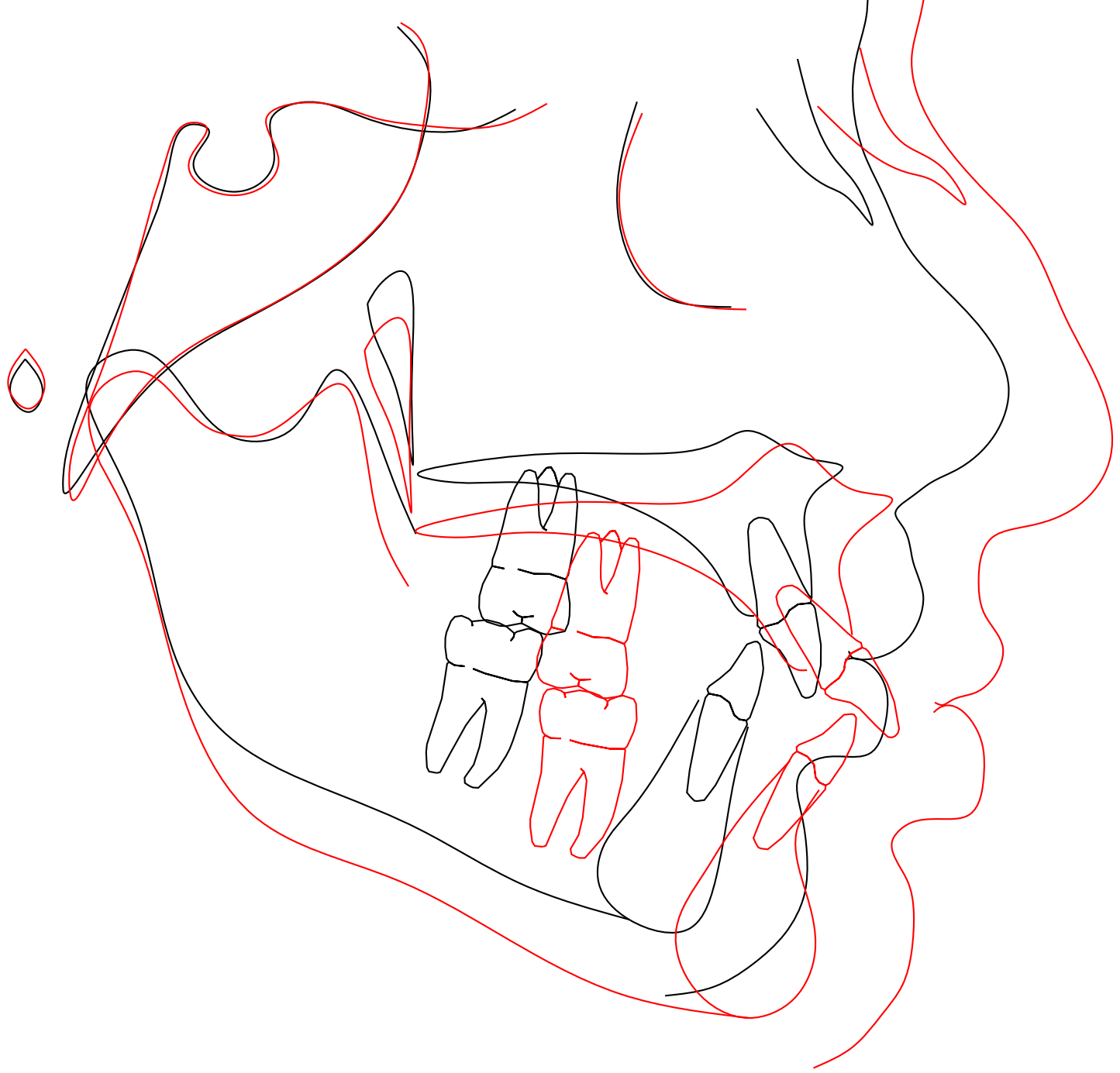

Figure F-23. Print of cephalometric tracing superimposition subject 23: (tracing is 1:1 with original radiograph). 


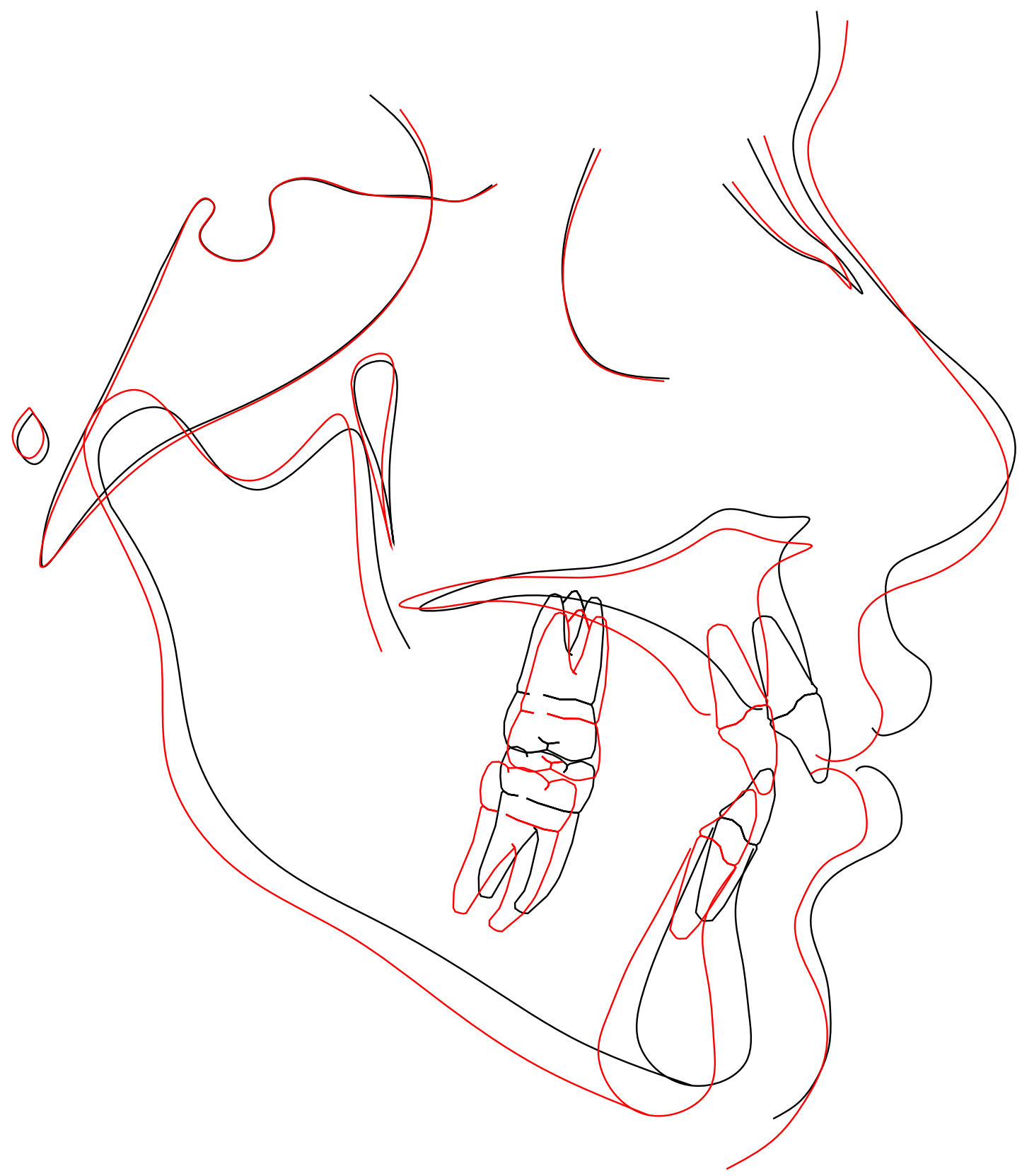

Figure F-24. Print of cephalometric tracing superimposition subject 24: (tracing is 1:1 with original radiograph). 


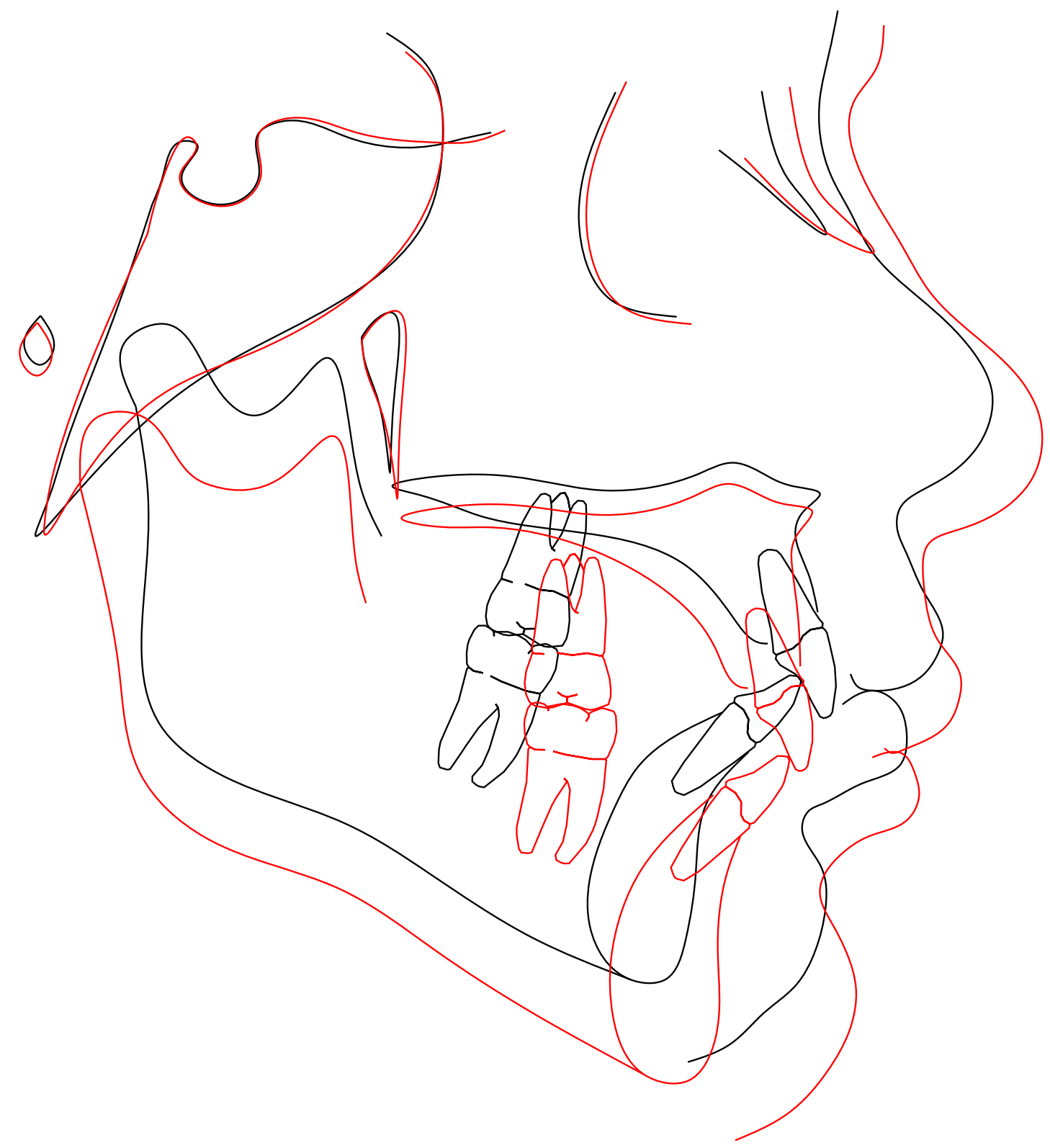

Figure F-25. Print of cephalometric tracing superimposition subject 25: (tracing is 1:1 with original radiograph). 


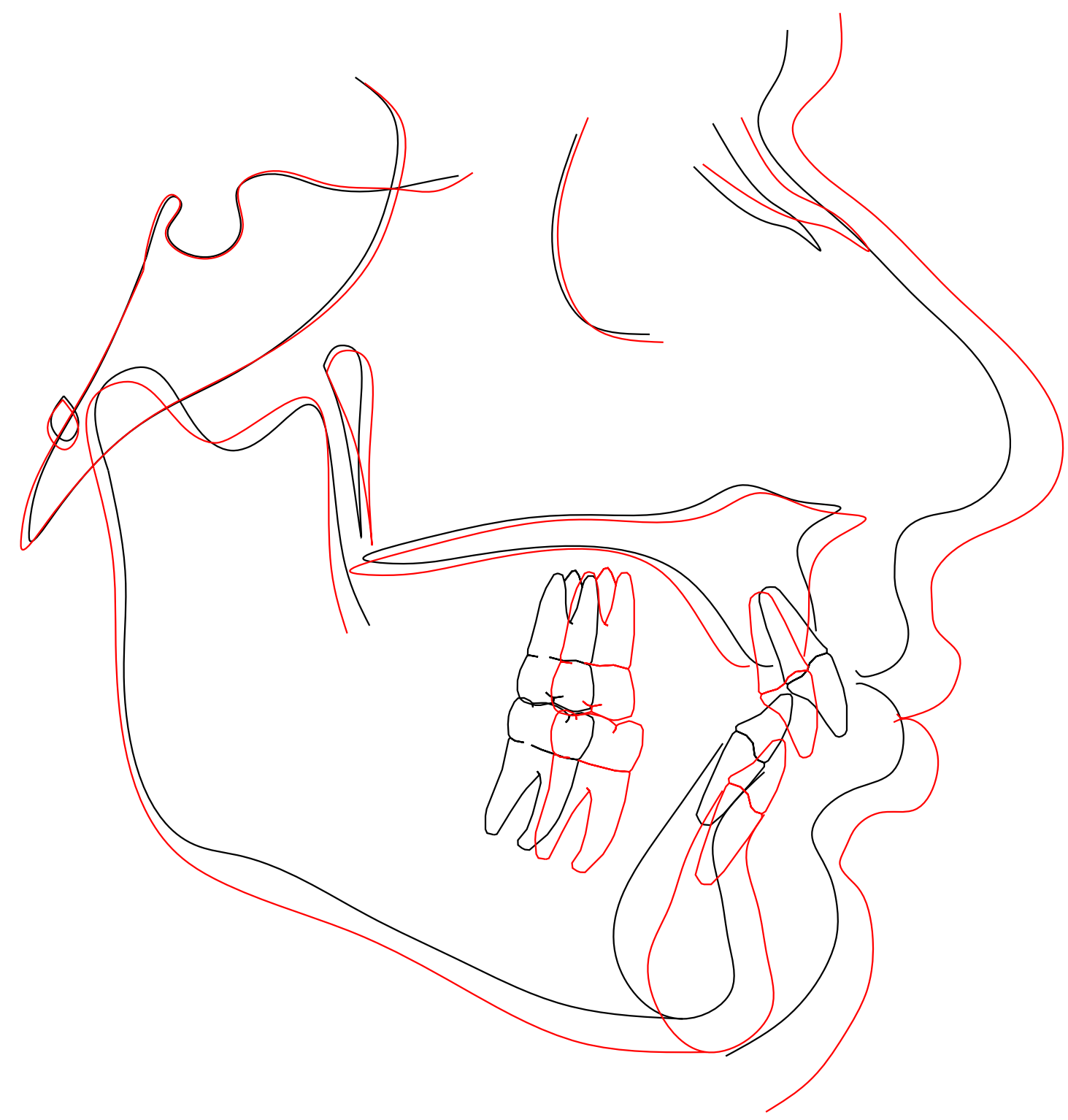

Figure F-26. Print of cephalometric tracing superimposition subject 26: (tracing is 1:1 with original radiograph). 


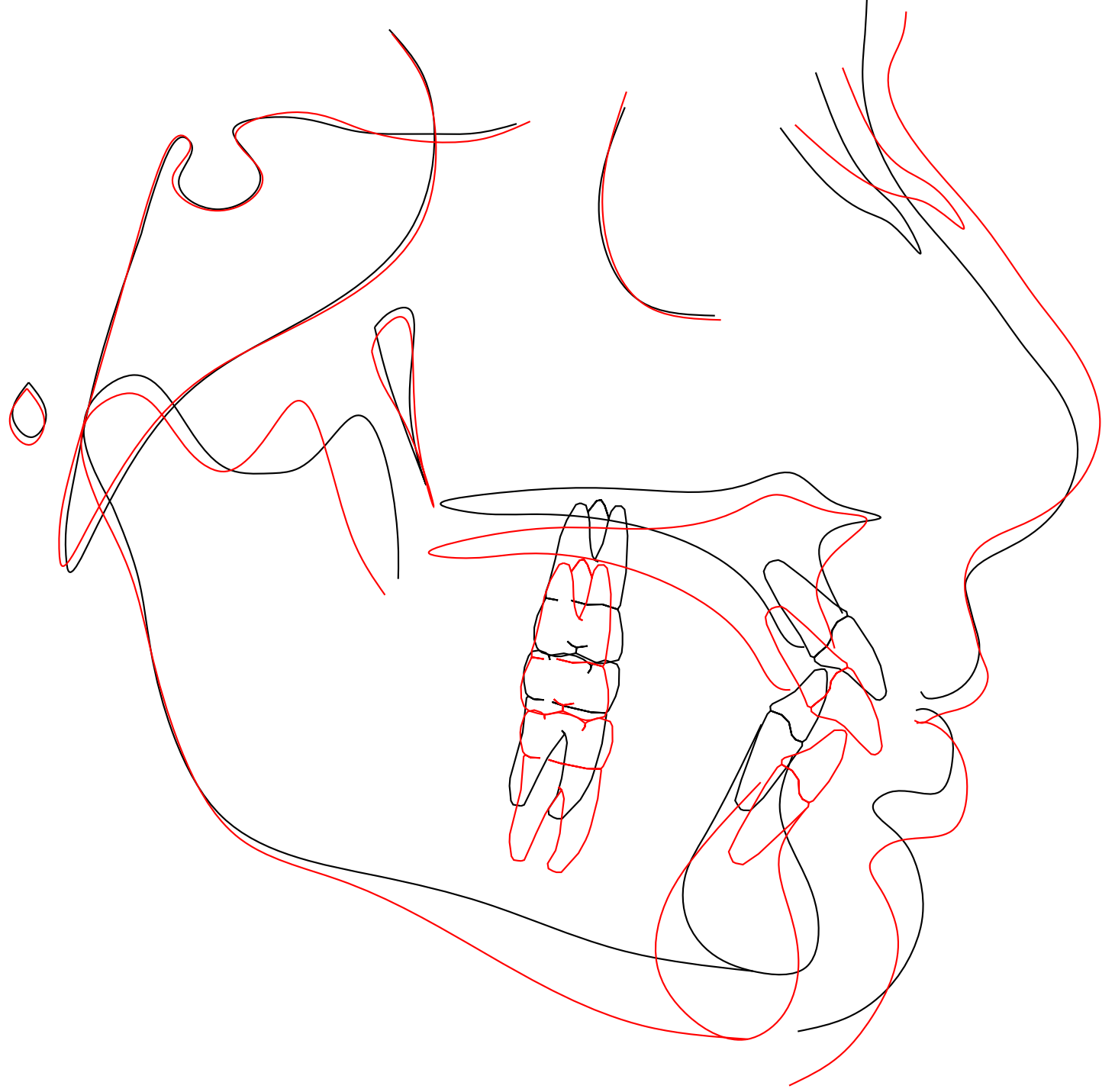

Figure F-27. Print of cephalometric tracing superimposition subject 27: (tracing is 1:1 with original radiograph). 


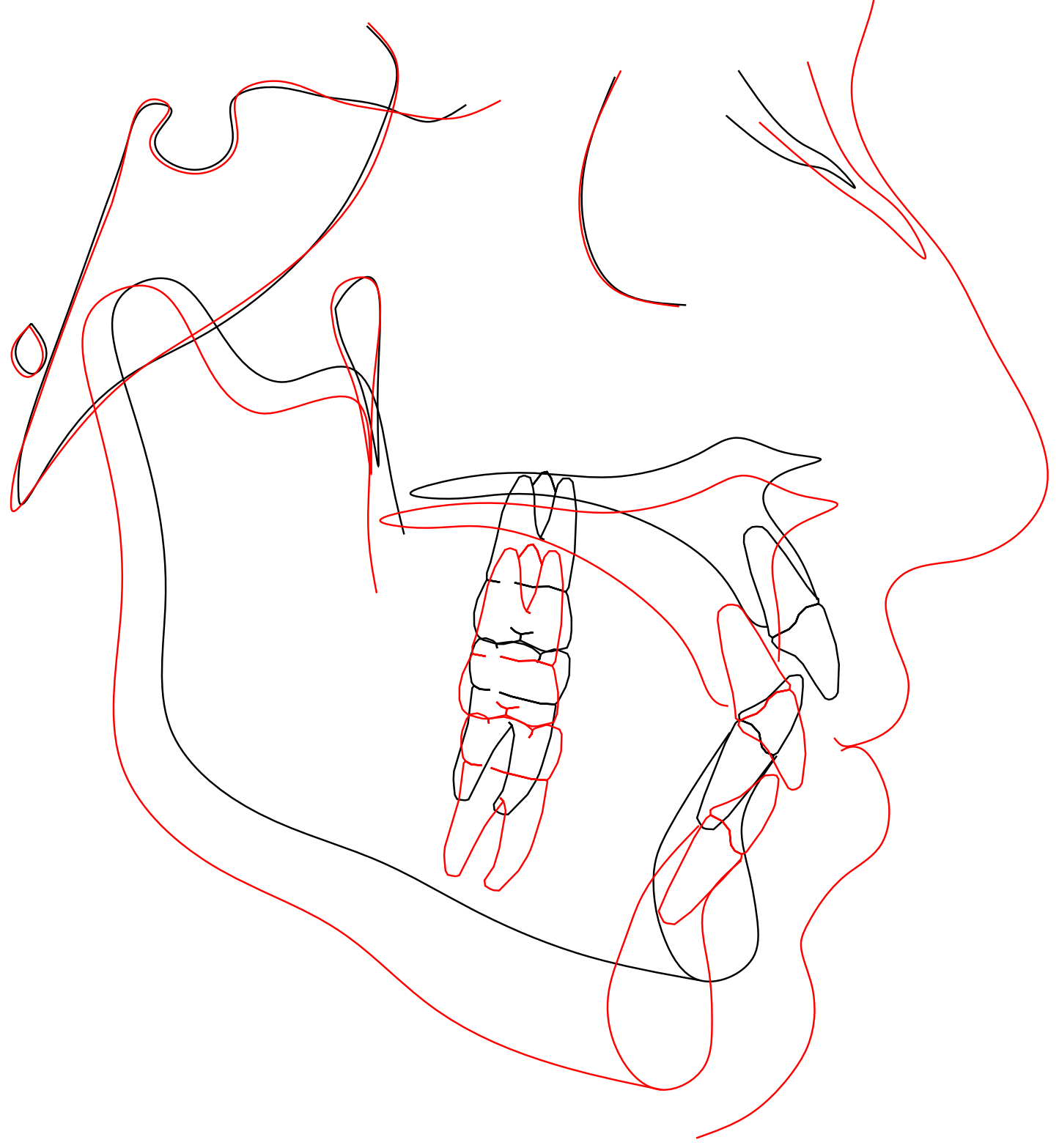

Figure F-28. Print of cephalometric tracing superimposition subject 28: (tracing is 1:1 with original radiograph). 


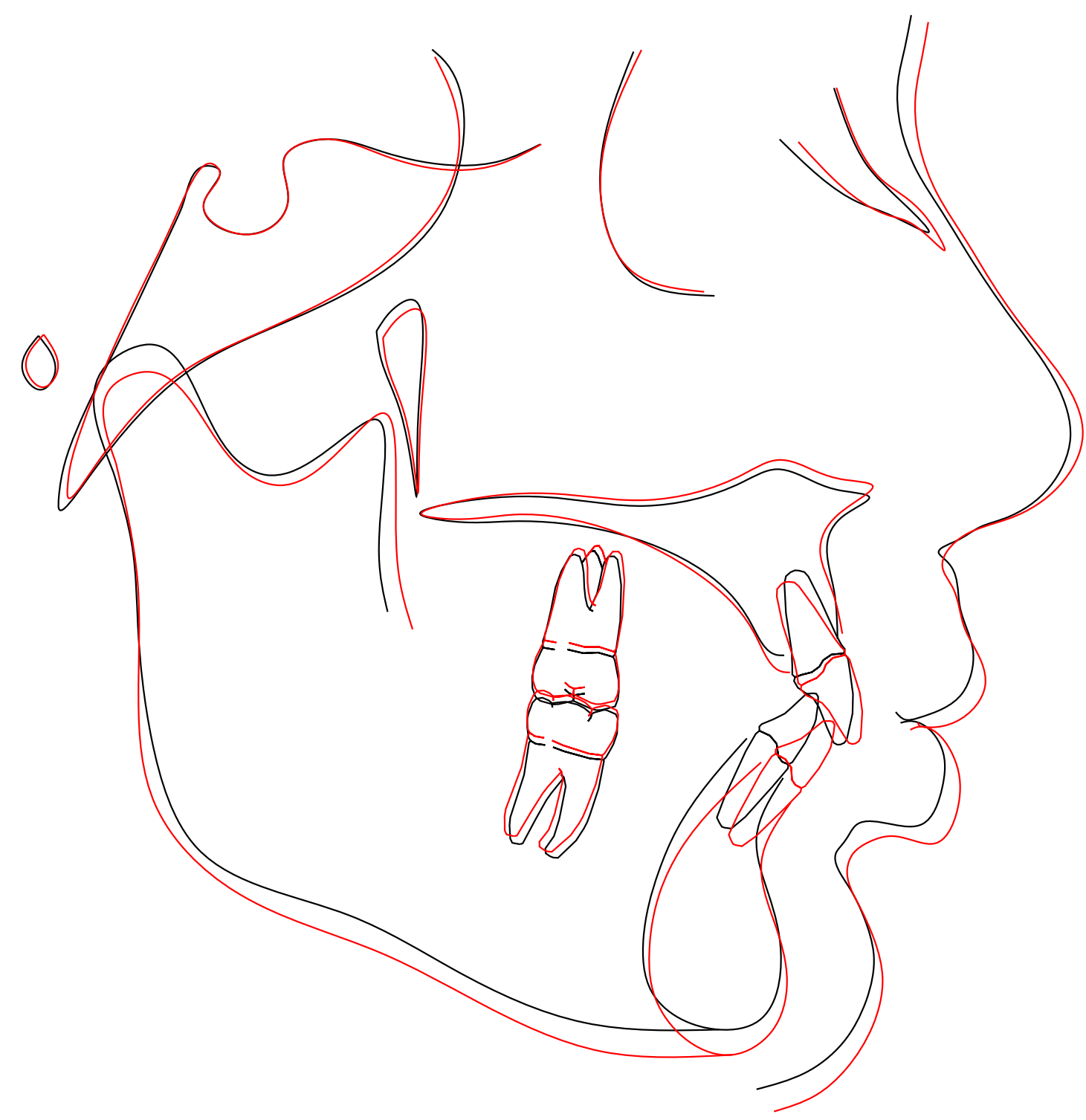

Figure F-29. Print of cephalometric tracing superimposition subject 29: (tracing is 1:1 with original radiograph). 


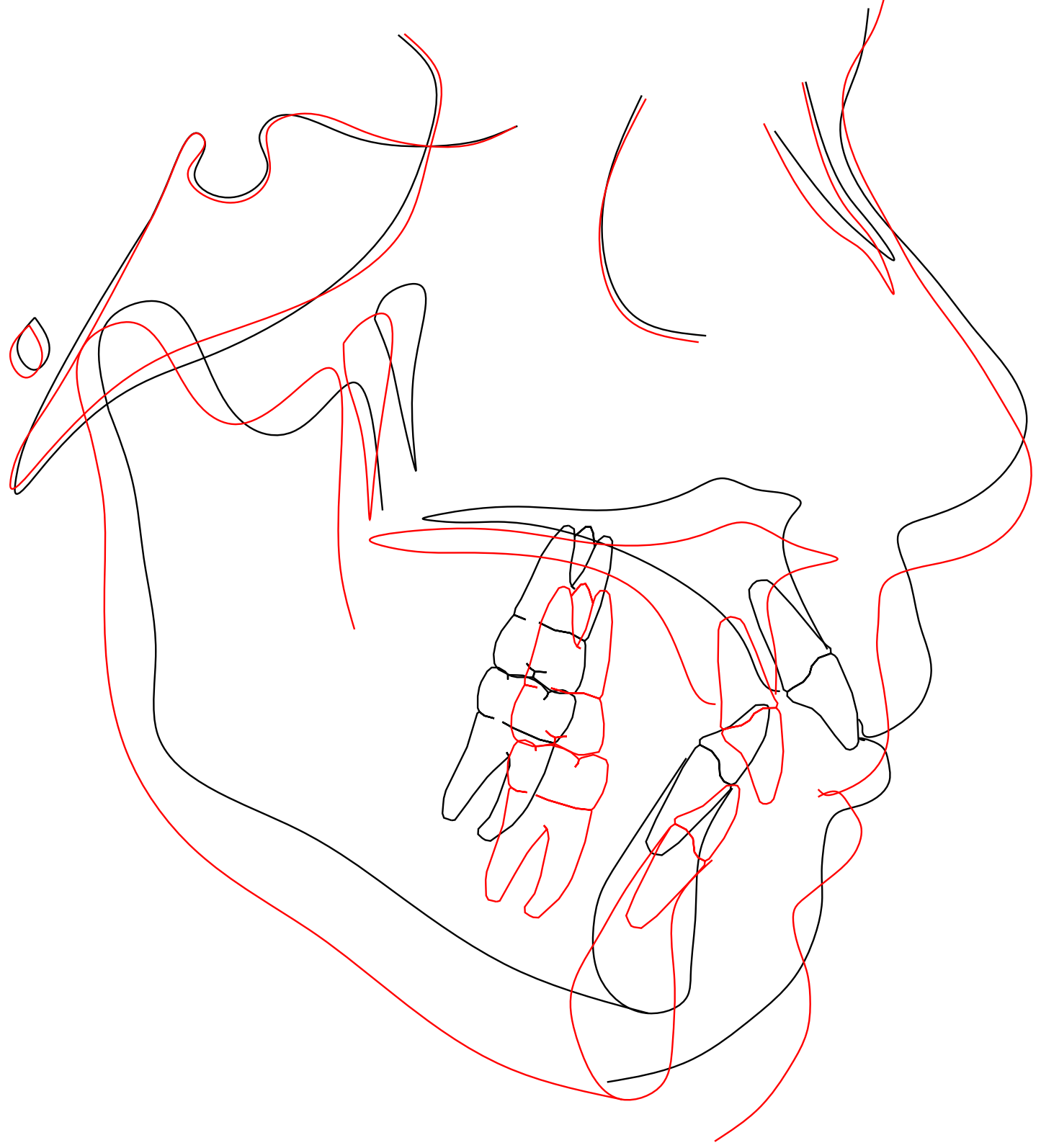

Figure F-30. Print of cephalometric tracing superimposition subject 30: (tracing is 1:1 with original radiograph). 


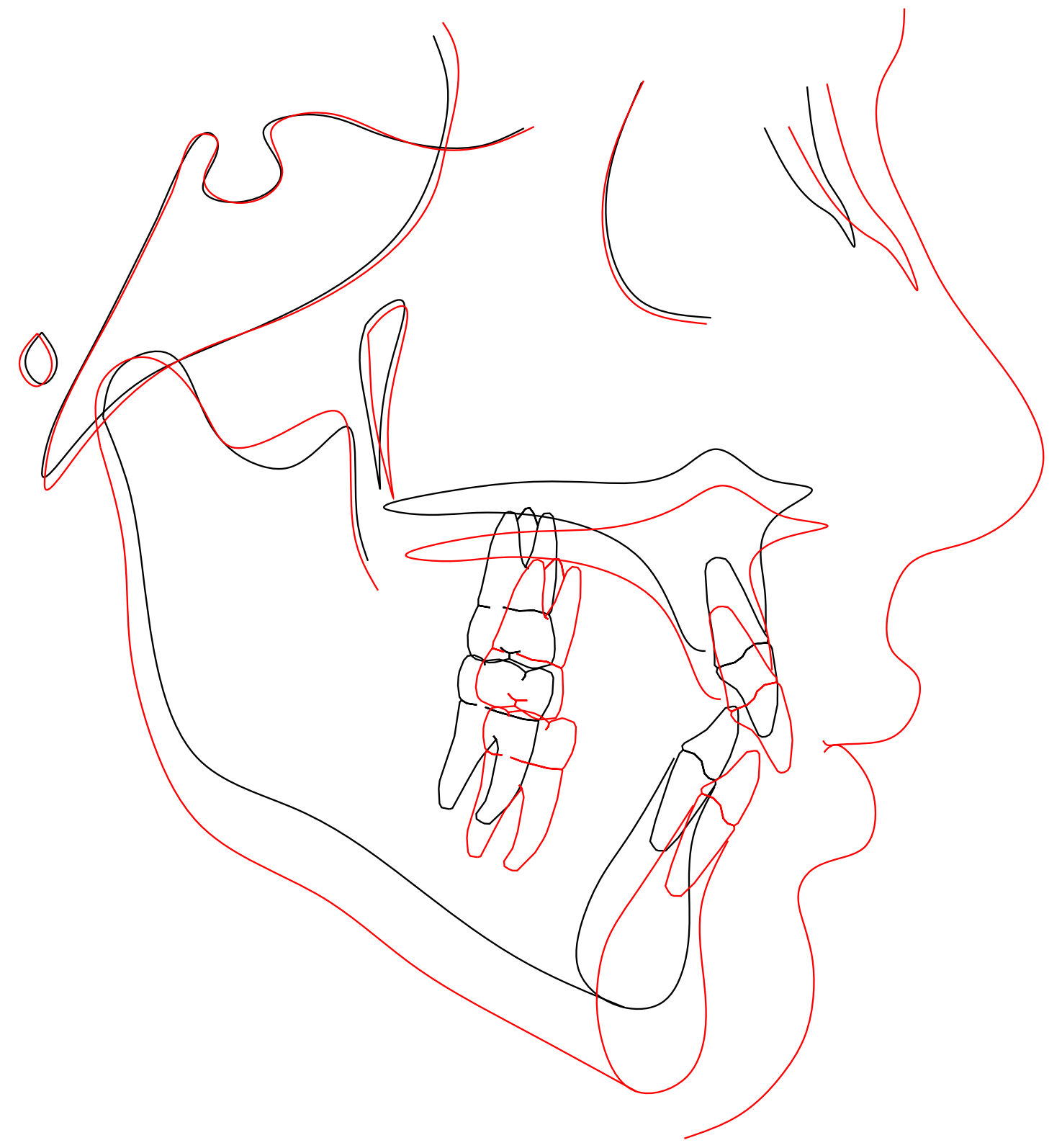

Figure F-31. Print of cephalometric tracing superimposition subject 31: (tracing is 1:1 with original radiograph). 


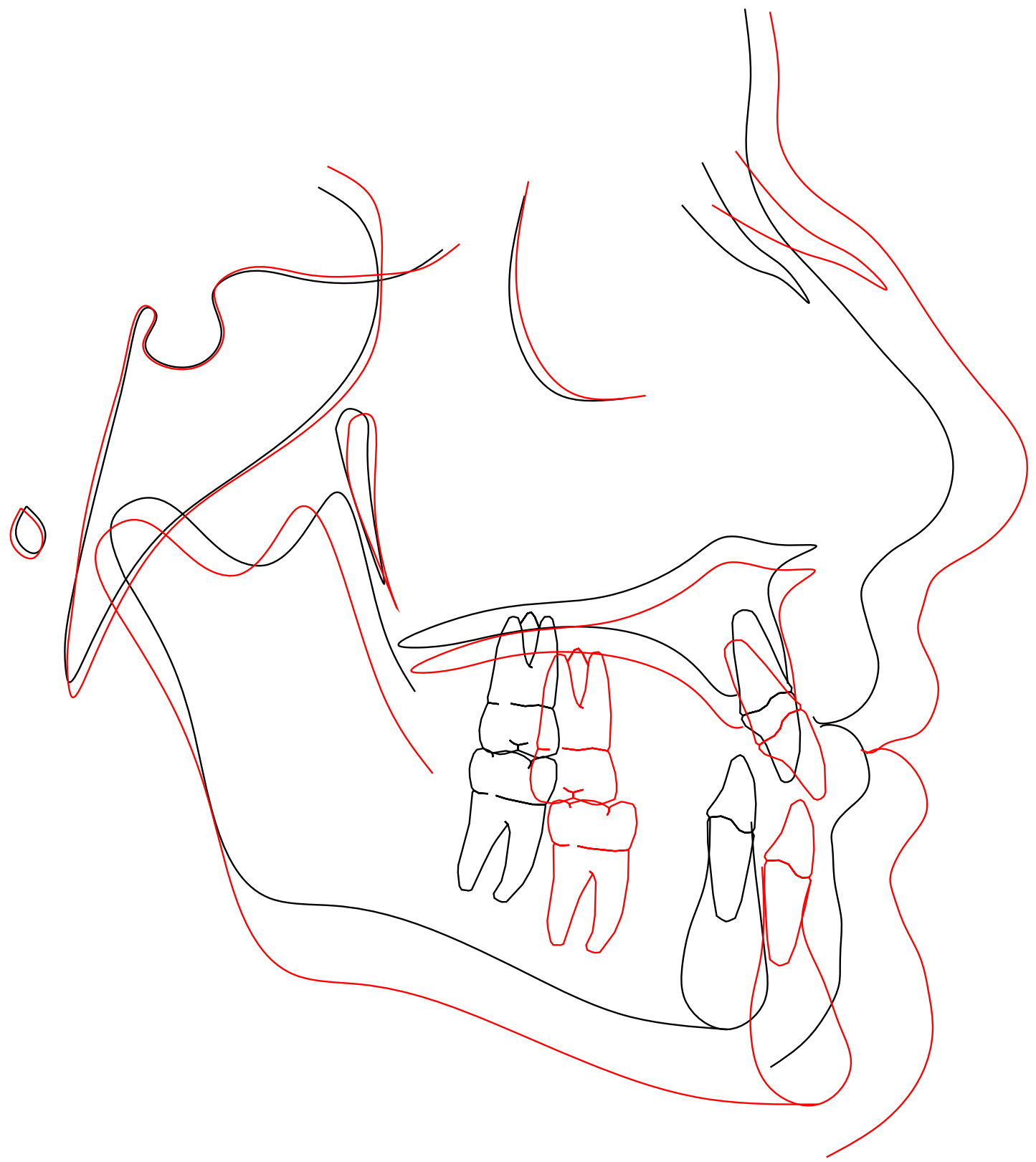

Figure F-32. Print of cephalometric tracing superimposition subject 32: (tracing is 1:1 with original radiograph). 


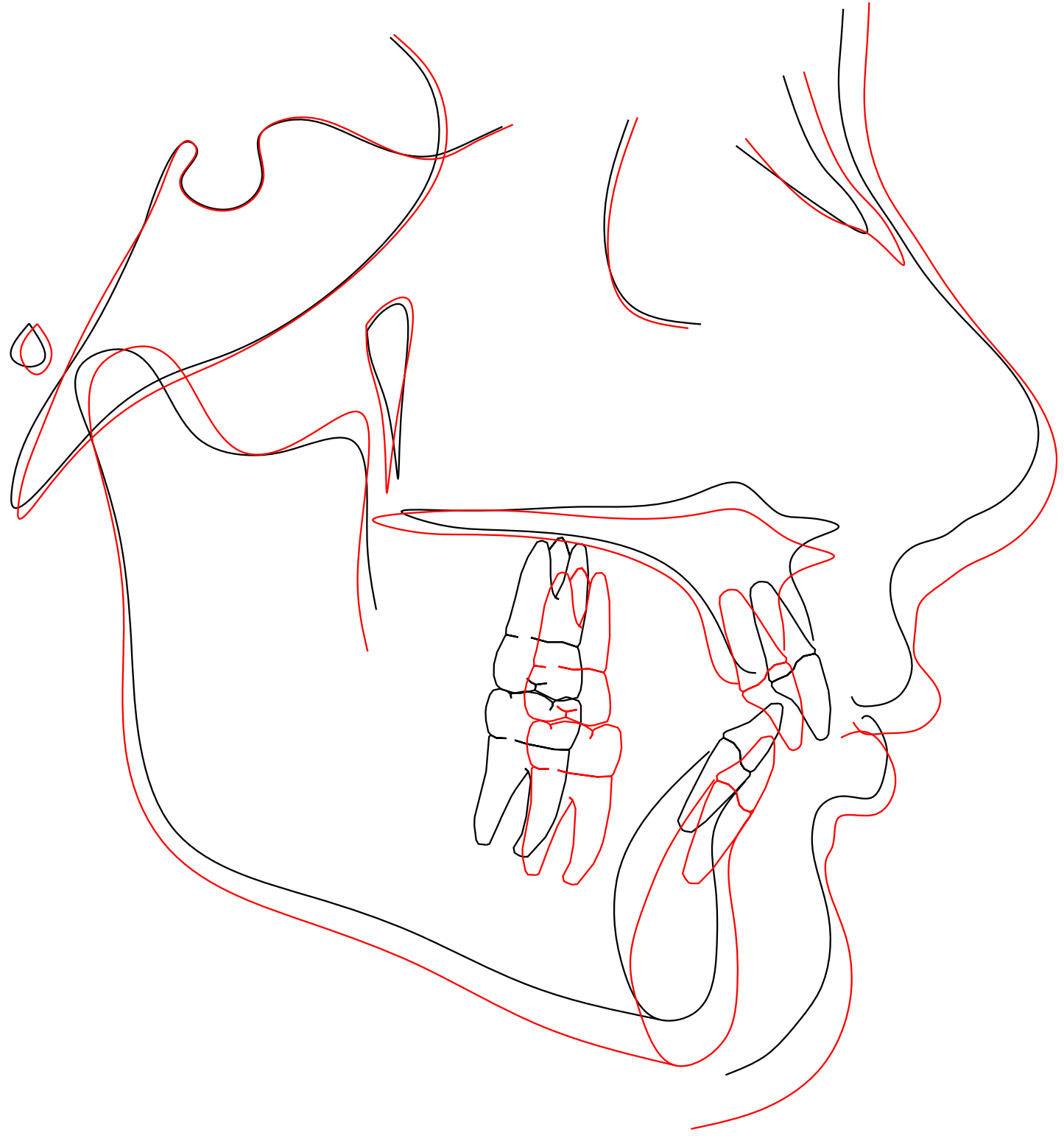

Figure F-33. Print of cephalometric tracing superimposition subject 33: (tracing is 1:1 with original radiograph). 


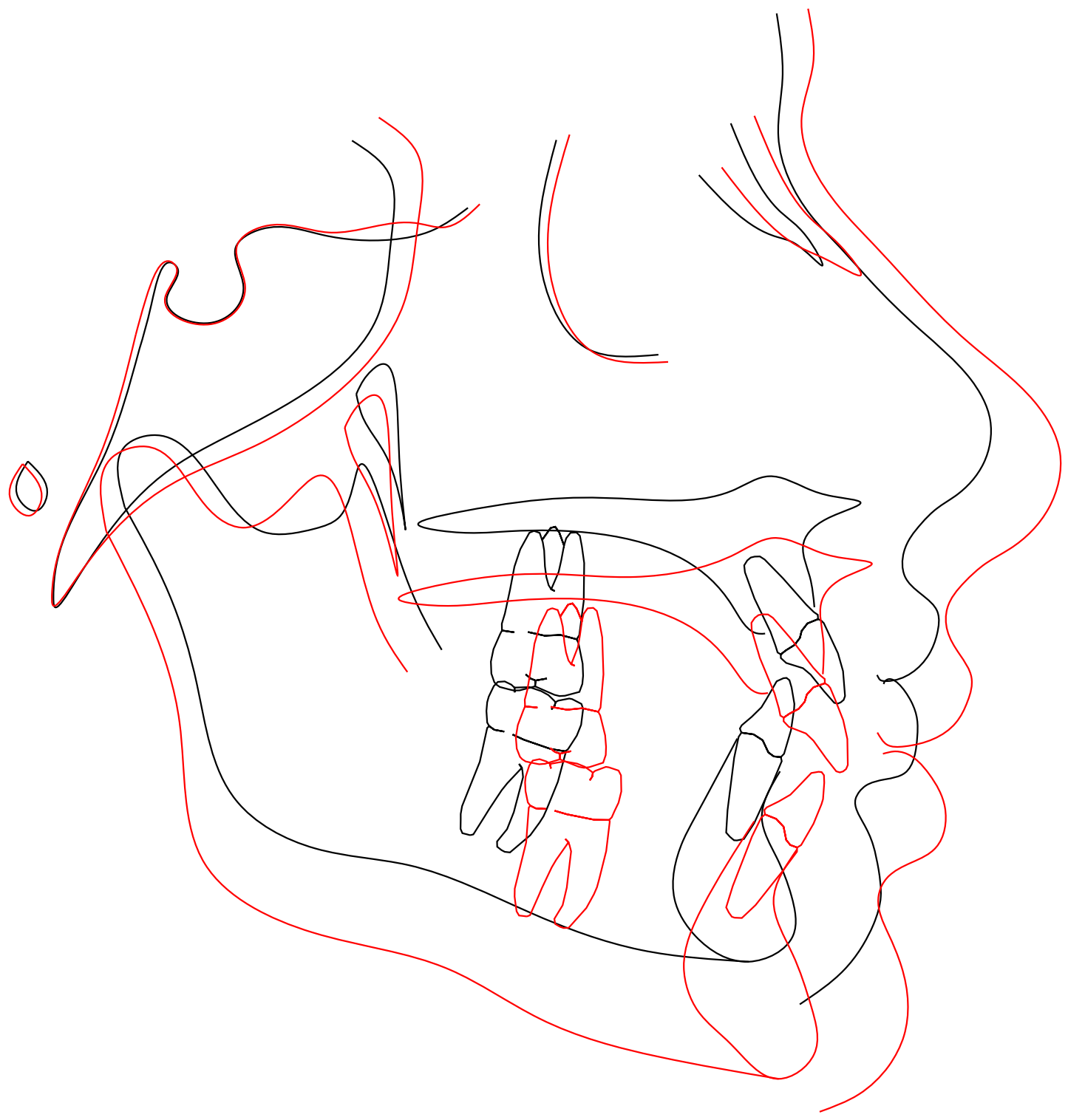

Figure F-34. Print of cephalometric tracing superimposition subject 34: (tracing is 1:1 with original radiograph). 


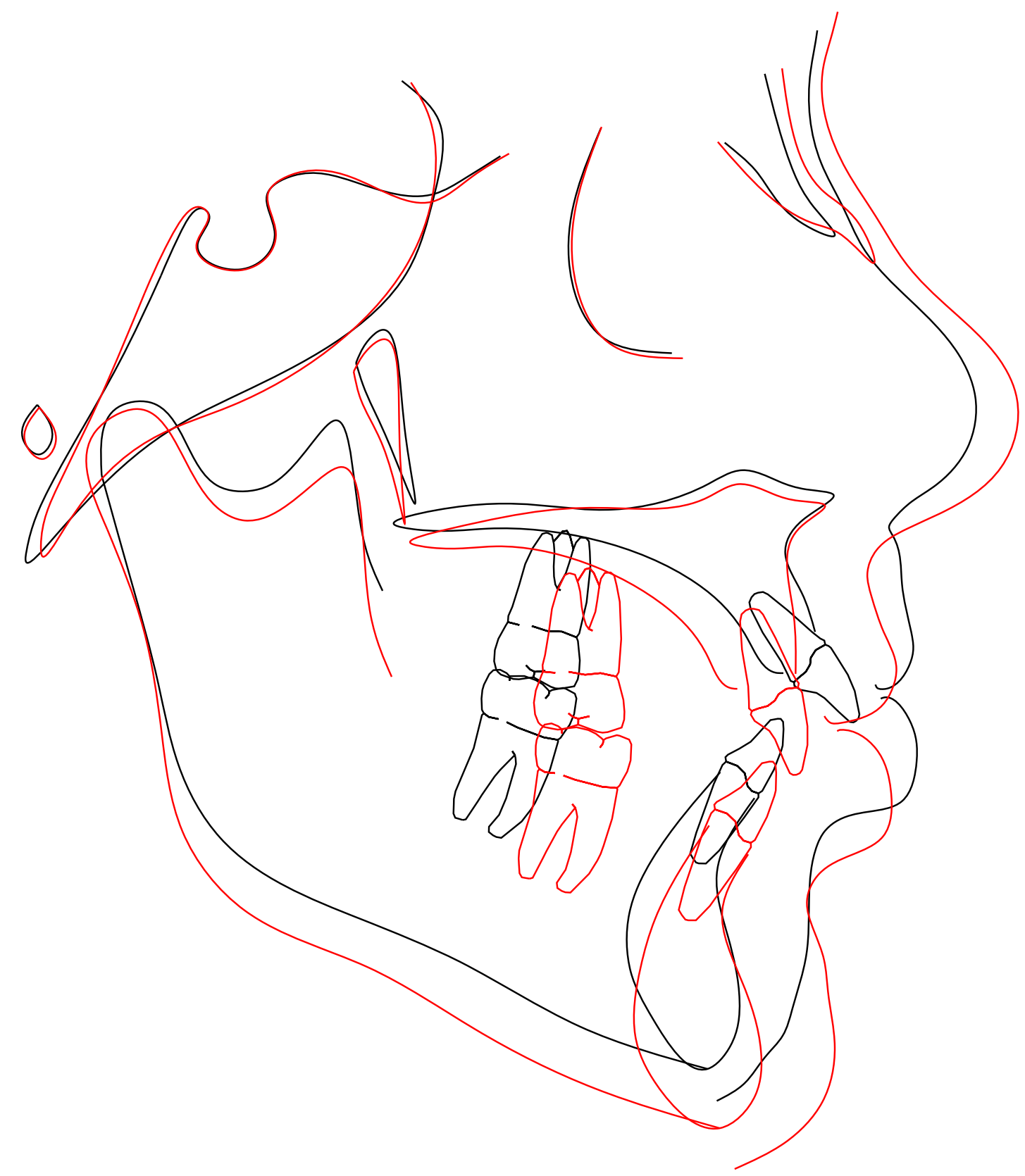

Figure F-35. Print of cephalometric tracing superimposition subject 35: (tracing is 1:1 with original radiograph). 


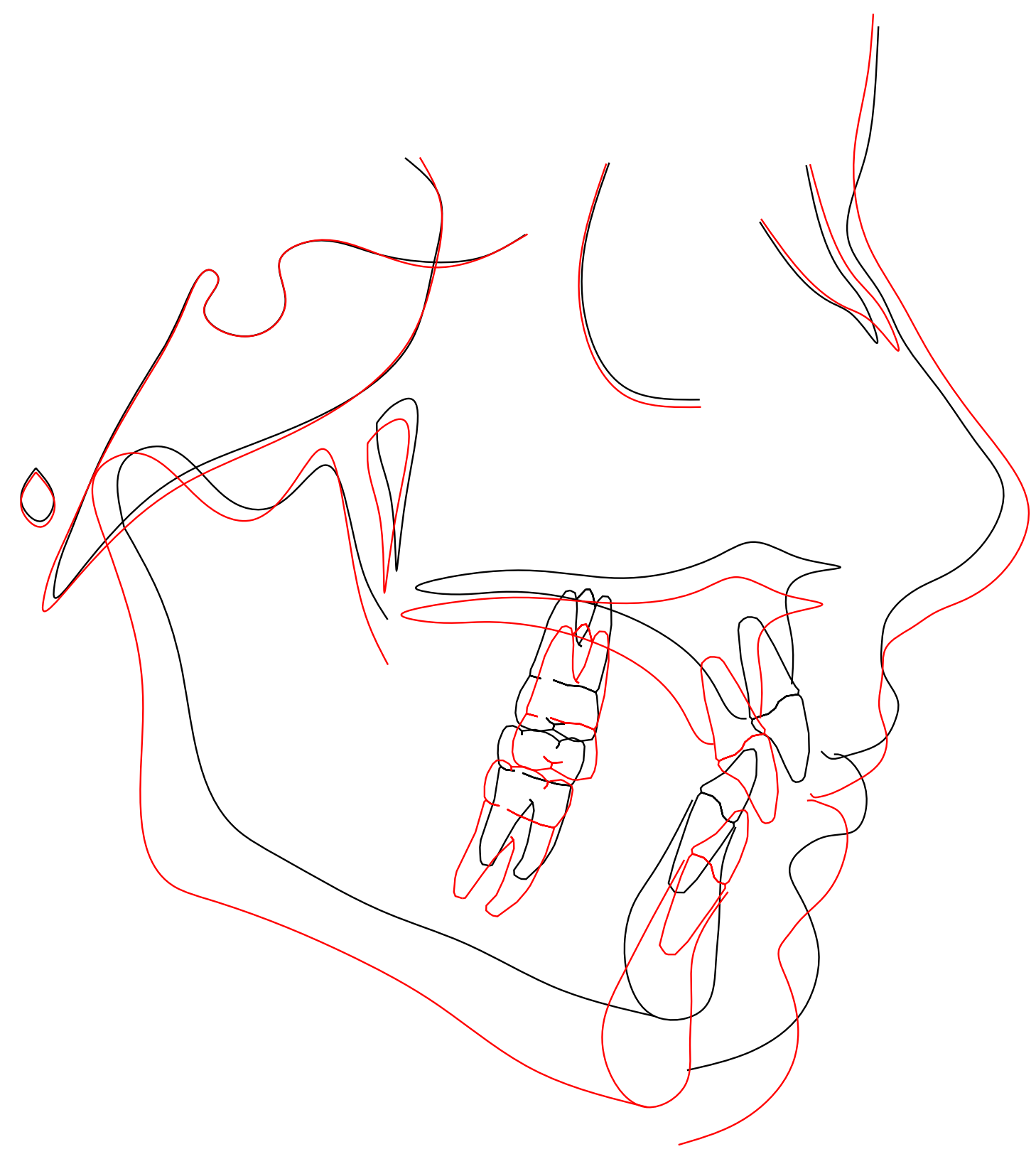

Figure F-36. Print of cephalometric tracing superimposition subject 36: (tracing is 1:1 with original radiograph). 


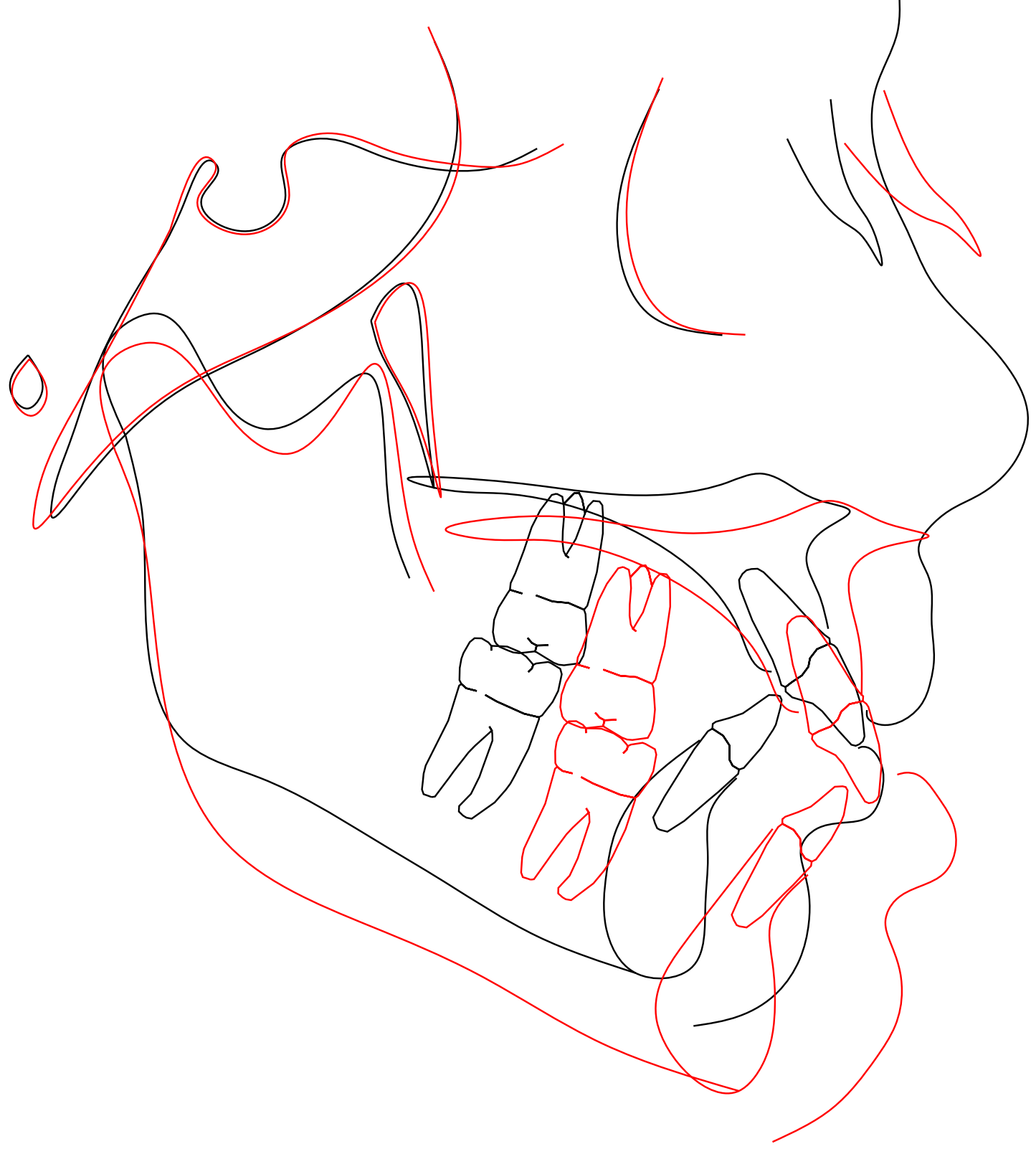

Figure F-37. Print of cephalometric tracing superimposition subject 37: (tracing is 1:1 with original radiograph). 


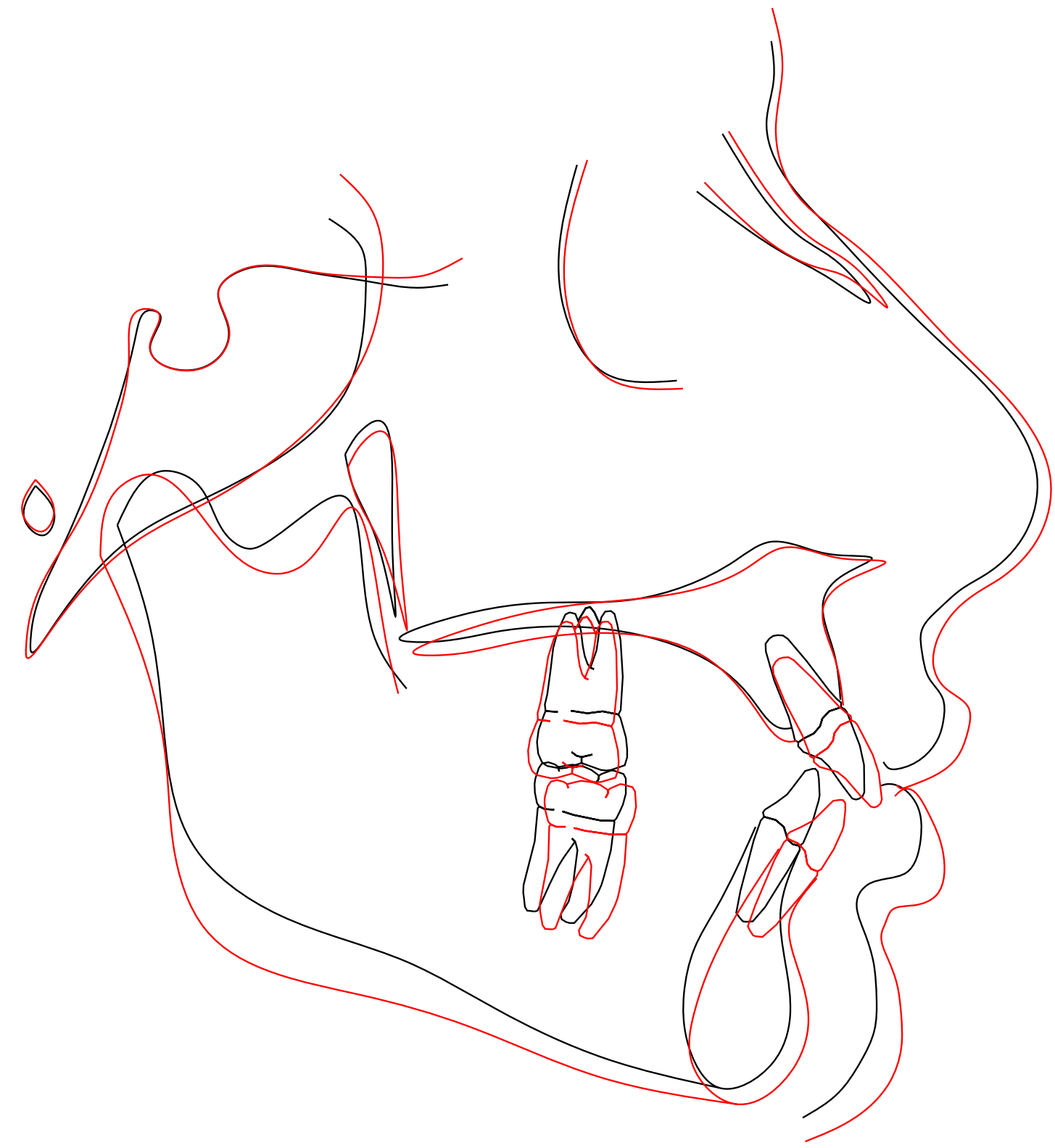

Figure F-38. Print of cephalometric tracing superimposition subject 38: (tracing is 1:1 with original radiograph). 


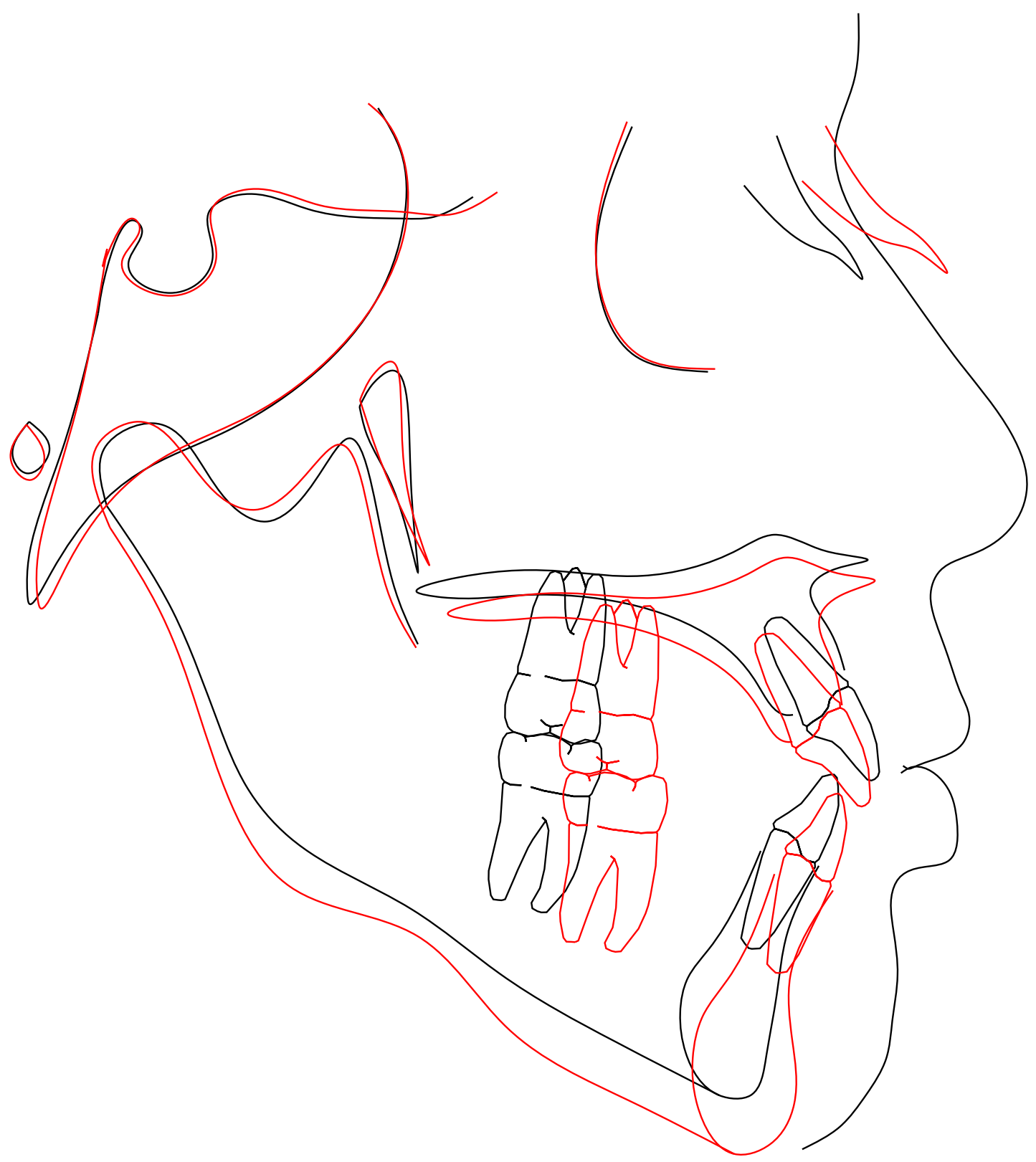

Figure F-39. Print of cephalometric tracing superimposition subject 39: (tracing is 1:1 with original radiograph). 


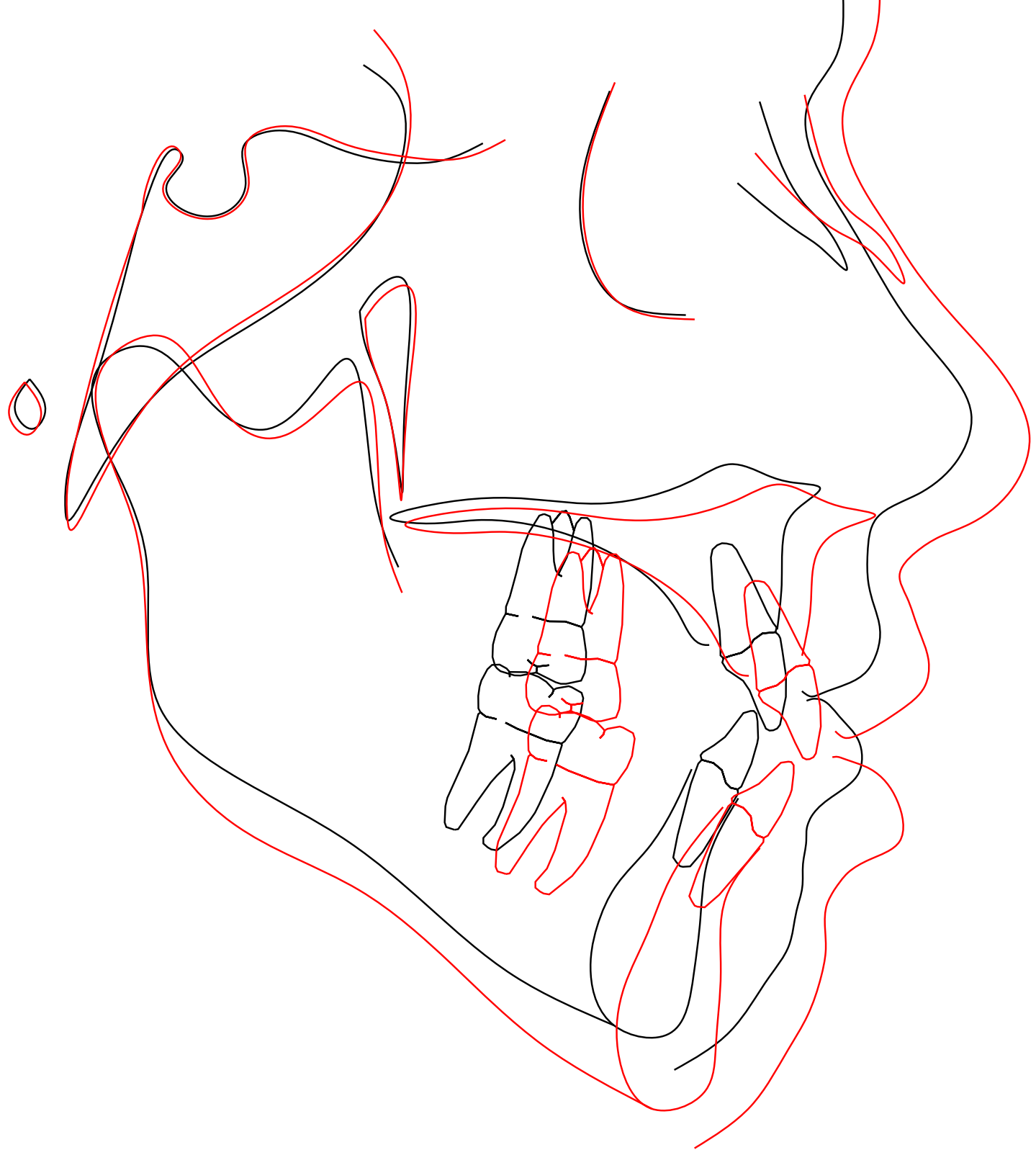

Figure F-40. Print of cephalometric tracing superimposition subject 40: (tracing is 1:1 with original radiograph). 


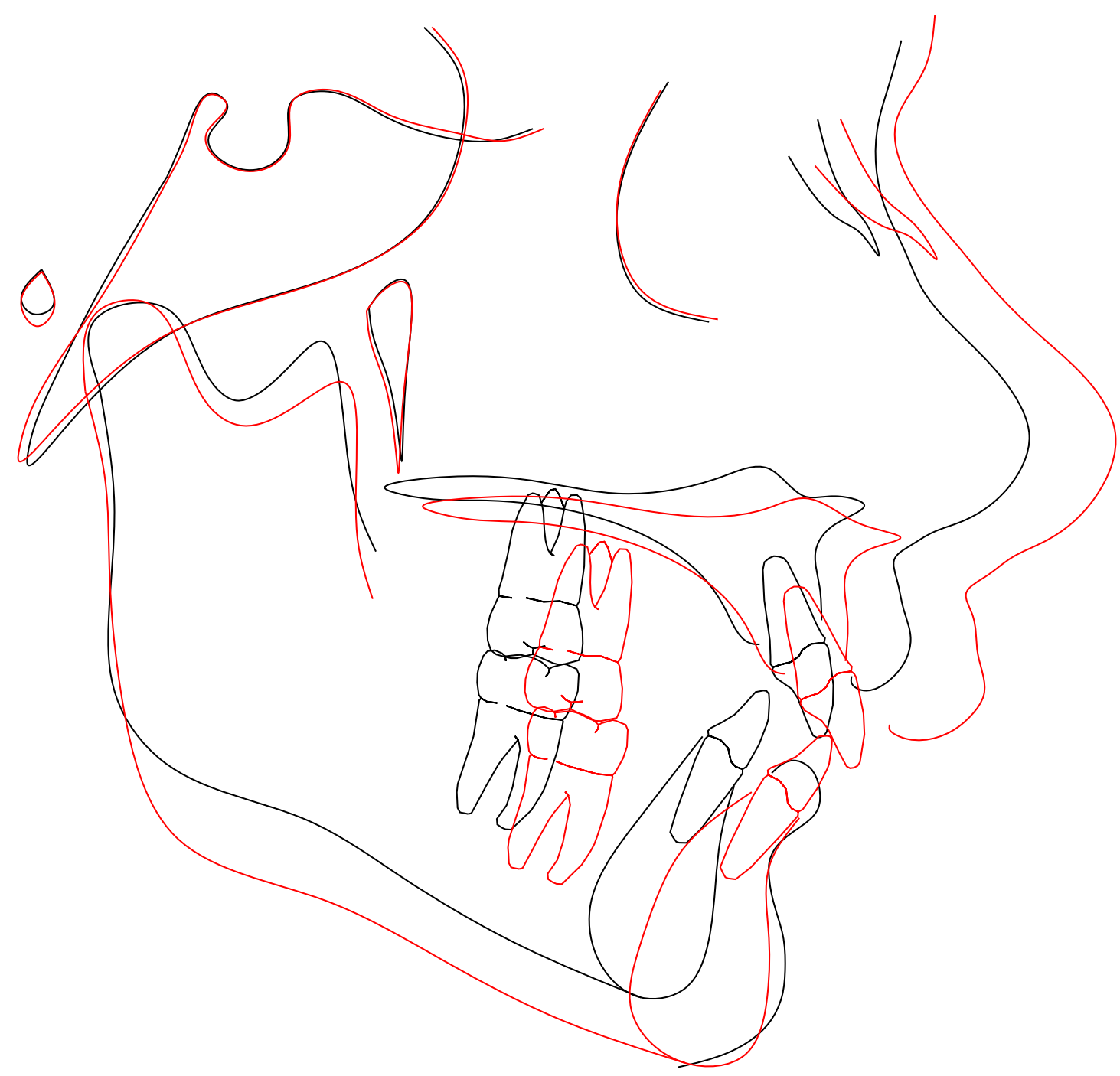

Figure F-41. Print of cephalometric tracing superimposition subject 41: (tracing is 1:1 with original radiograph). 


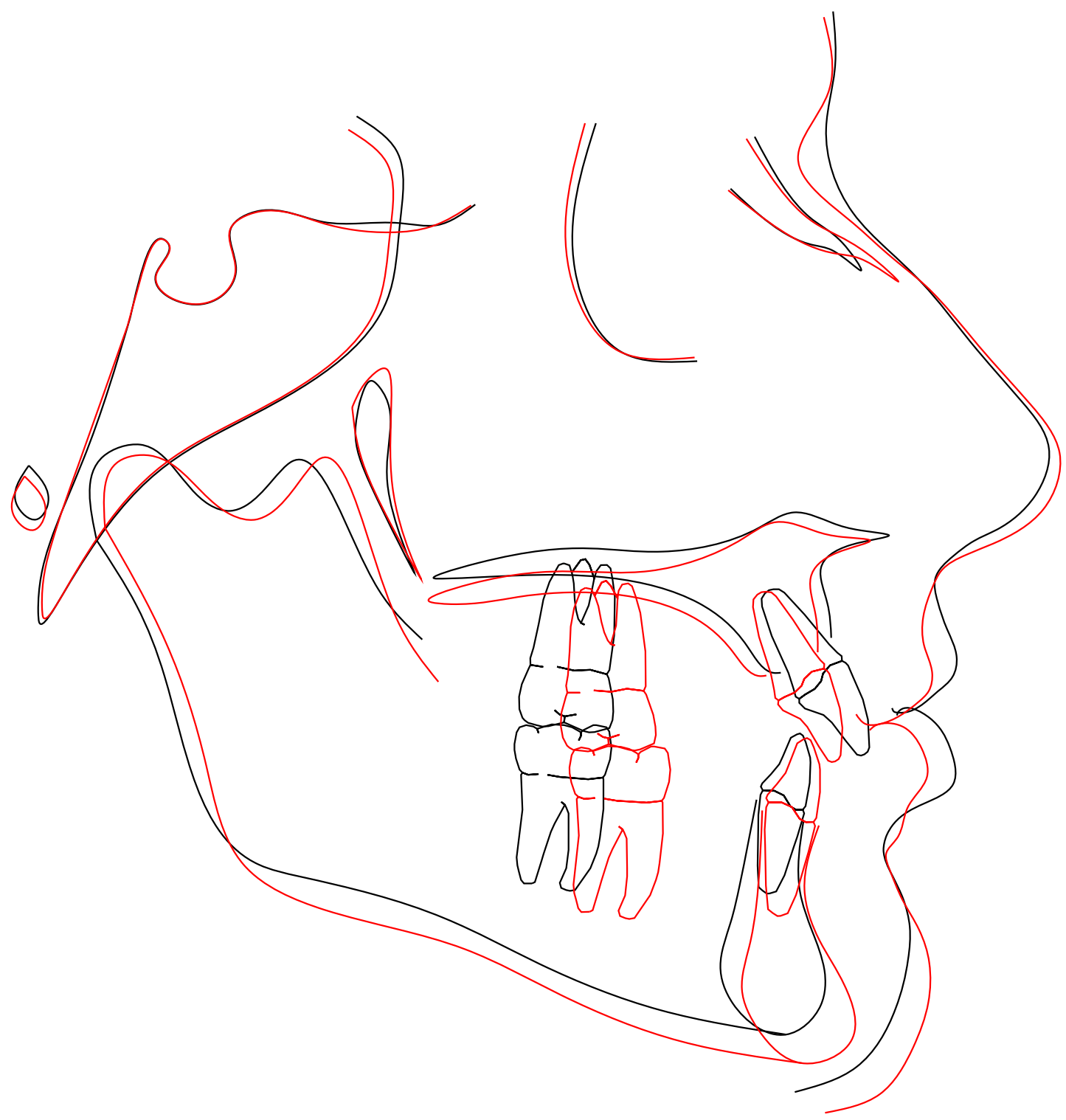

Figure F-42. Print of cephalometric tracing superimposition subject 42: (tracing is 1:1 with original radiograph). 


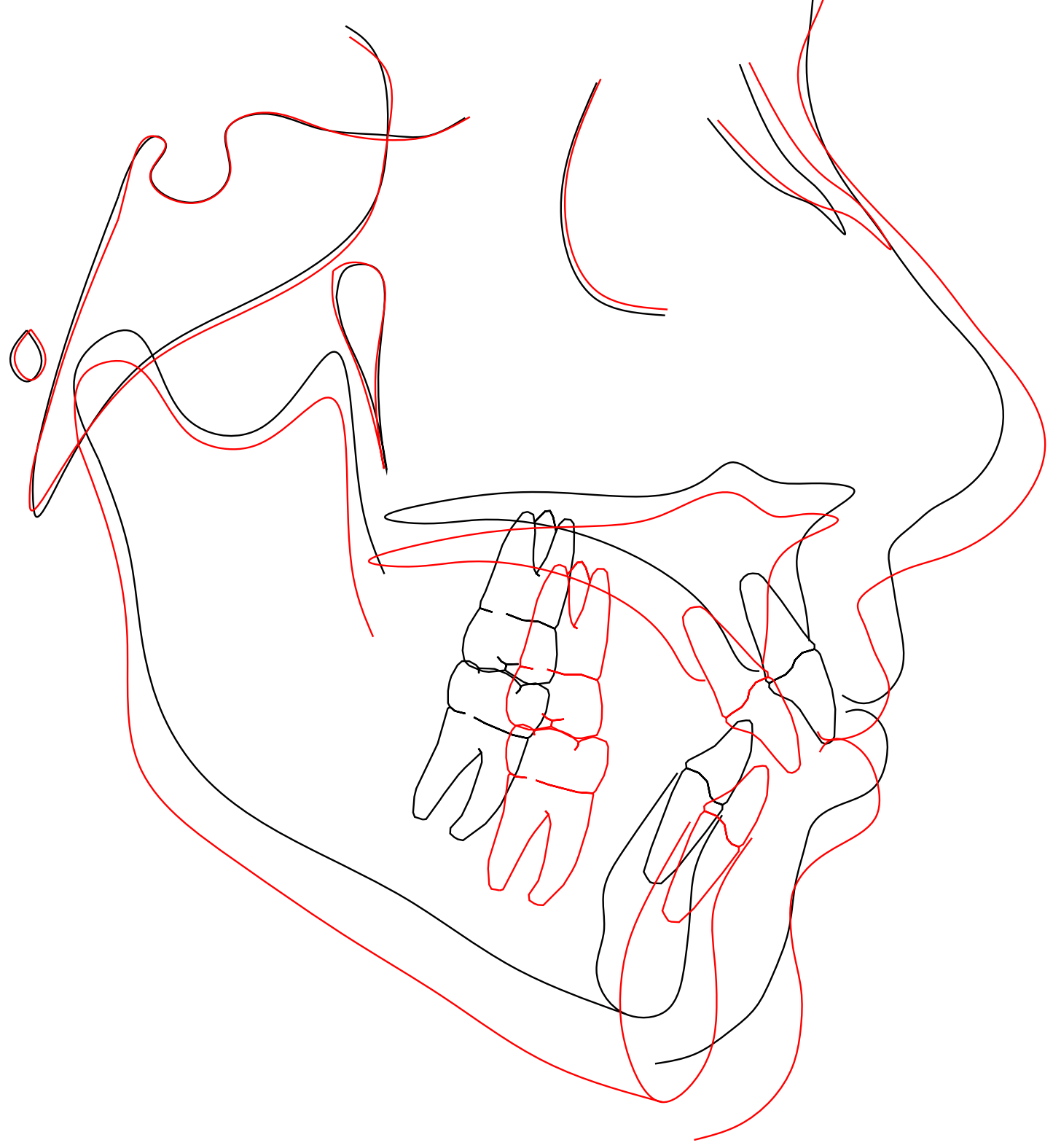

Figure F-43. Print of cephalometric tracing superimposition subject 43 (tracing is 1:1 with original radiograph). 


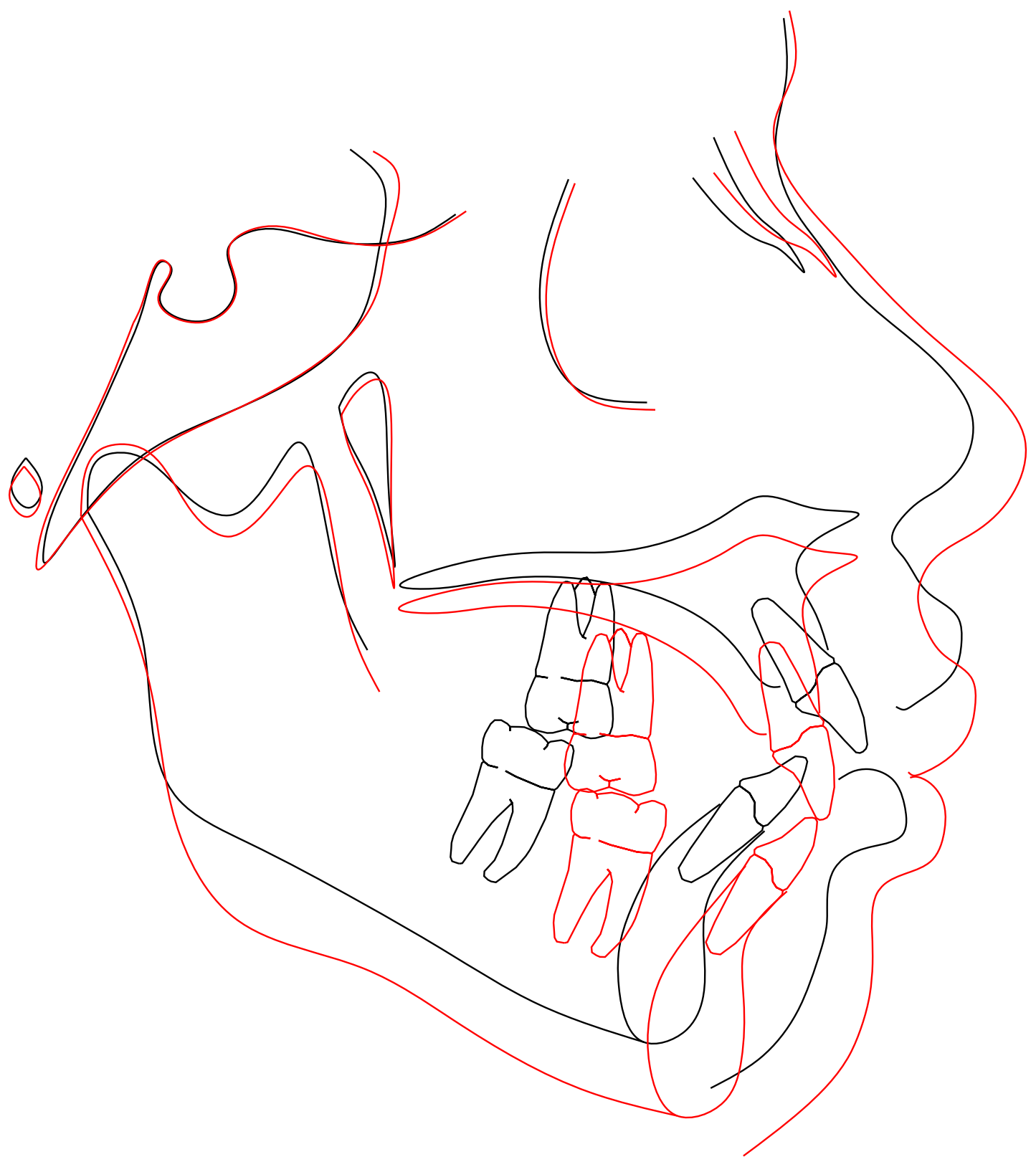

Figure F-44. Print of cephalometric tracing superimposition subject 44 (tracing is 1:1 with original radiograph). 


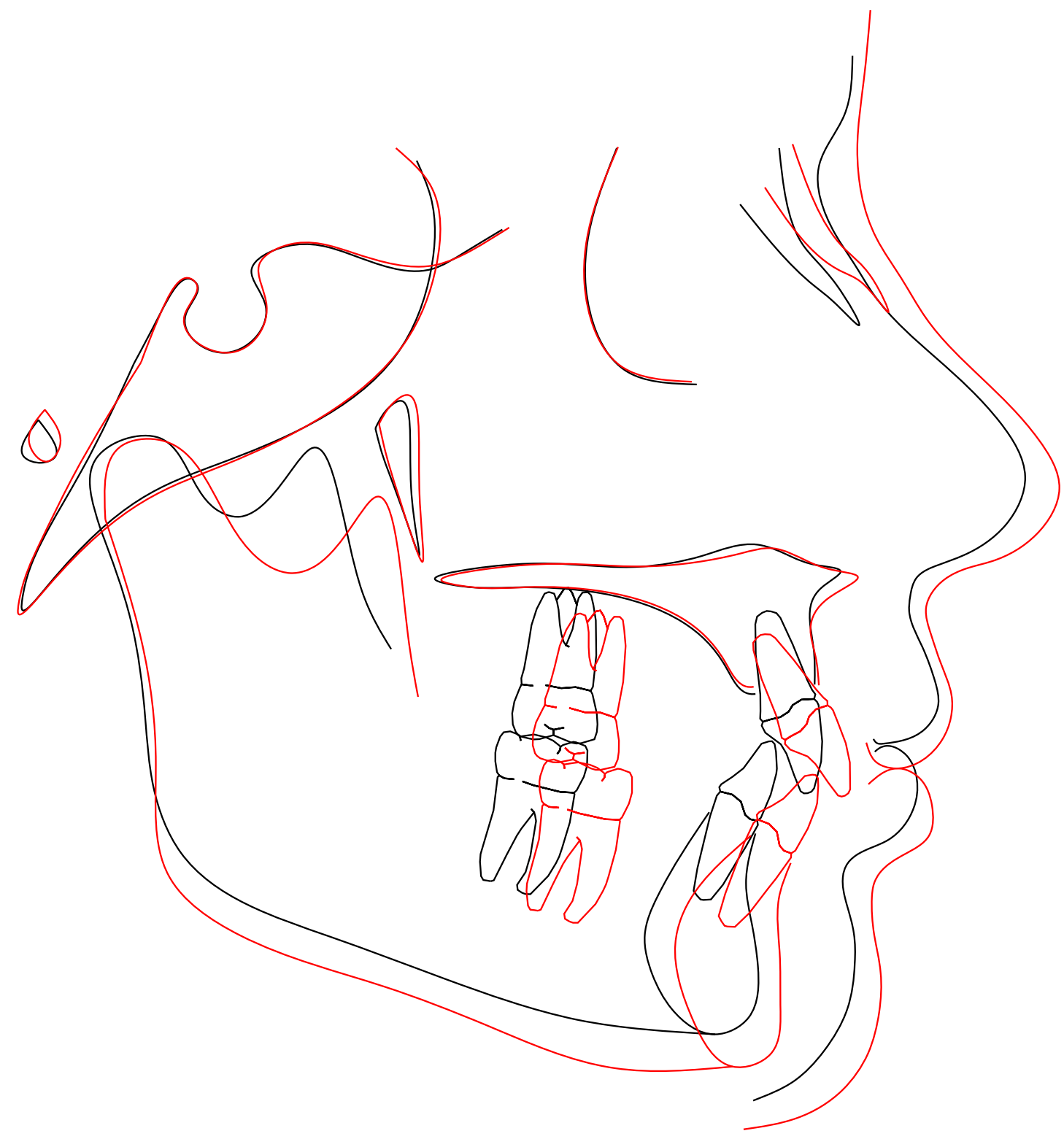

Figure F-45. Print of cephalometric tracing superimposition subject 45 (tracing is 1:1 with original radiograph). 


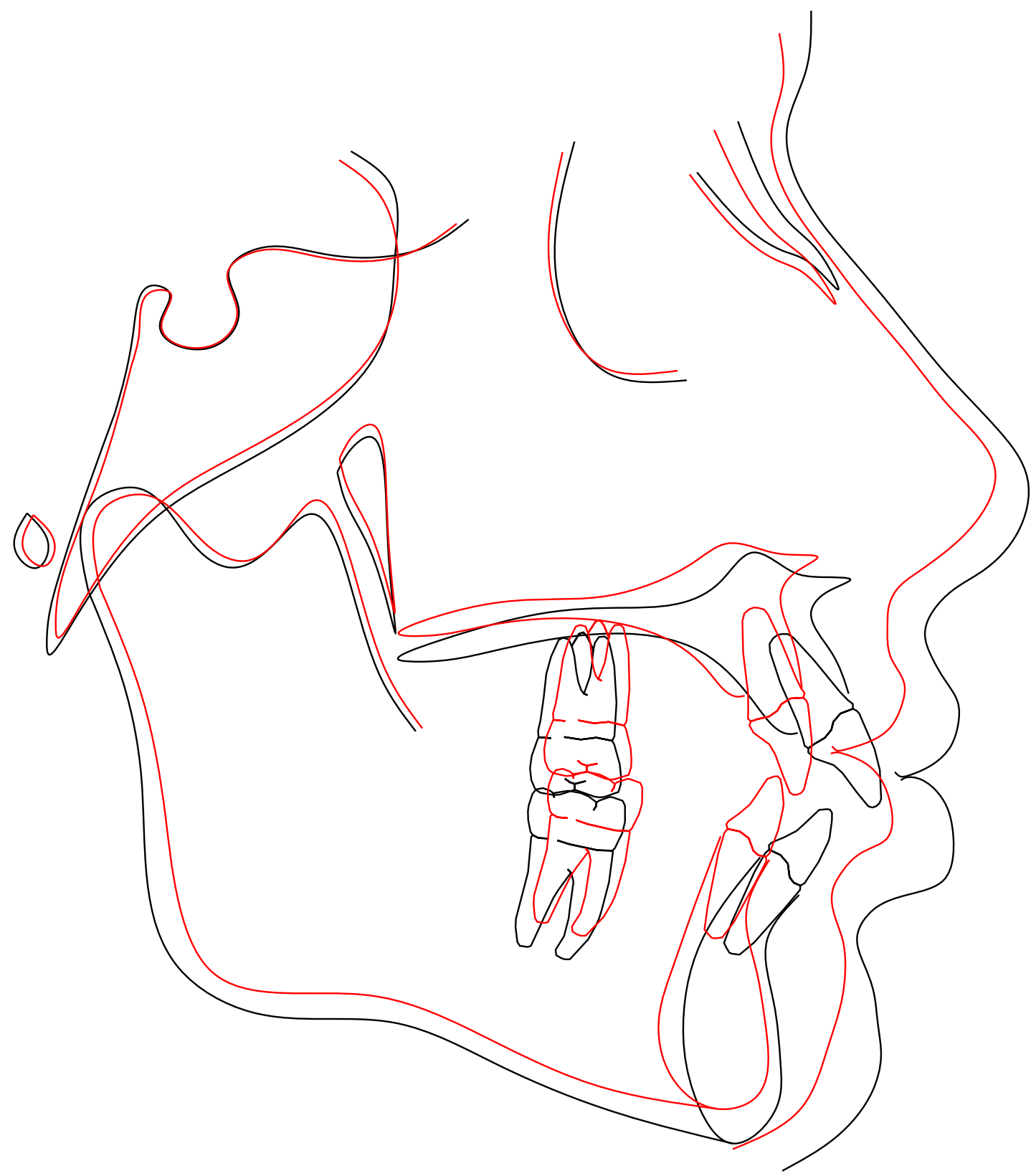

Figure F-46. Print of cephalometric tracing superimposition subject 46 (tracing is 1:1 with original radiograph). 


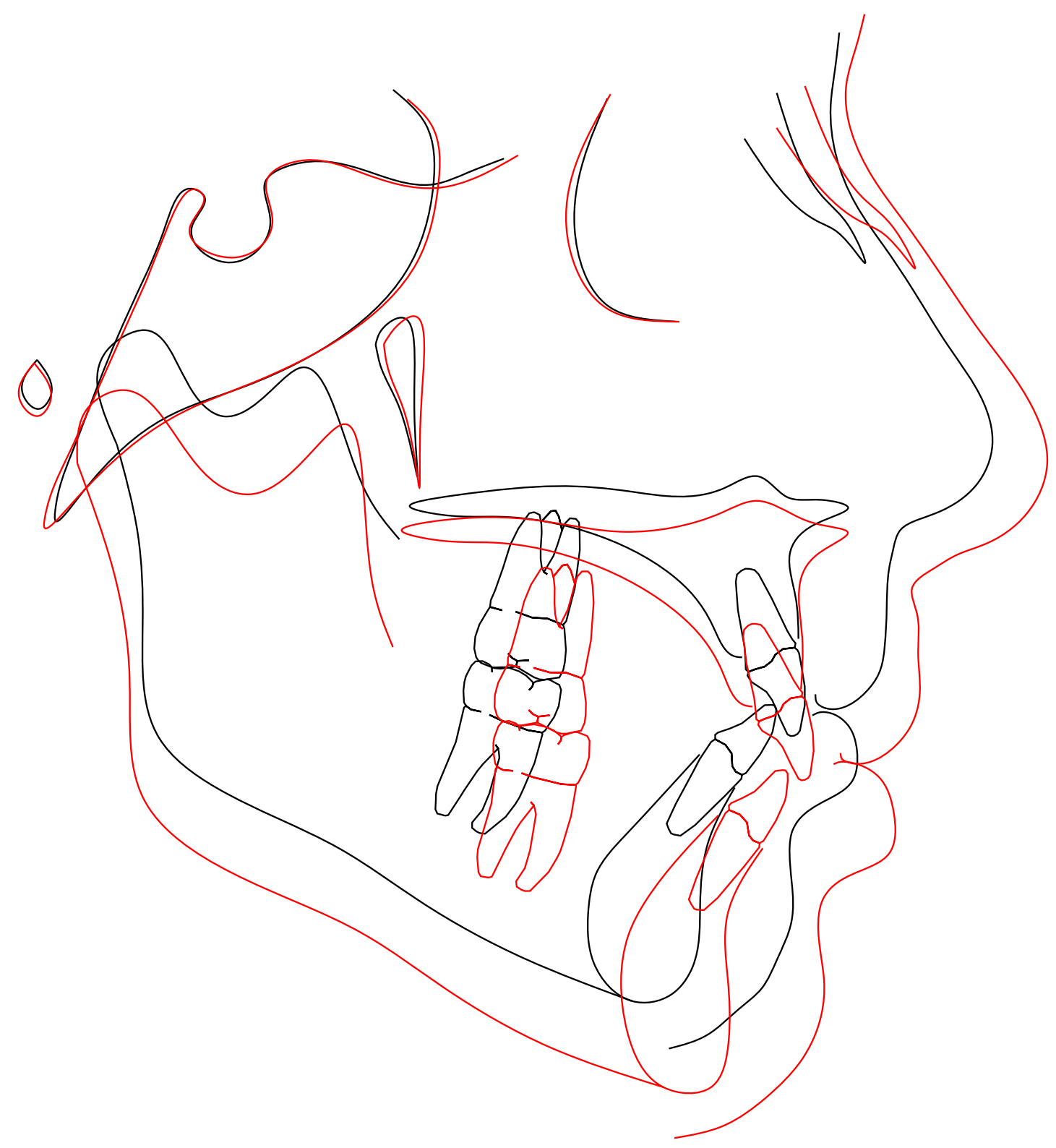

Figure F-47. Print of cephalometric tracing superimposition subject 47 (tracing is 1:1 with original radiograph). 


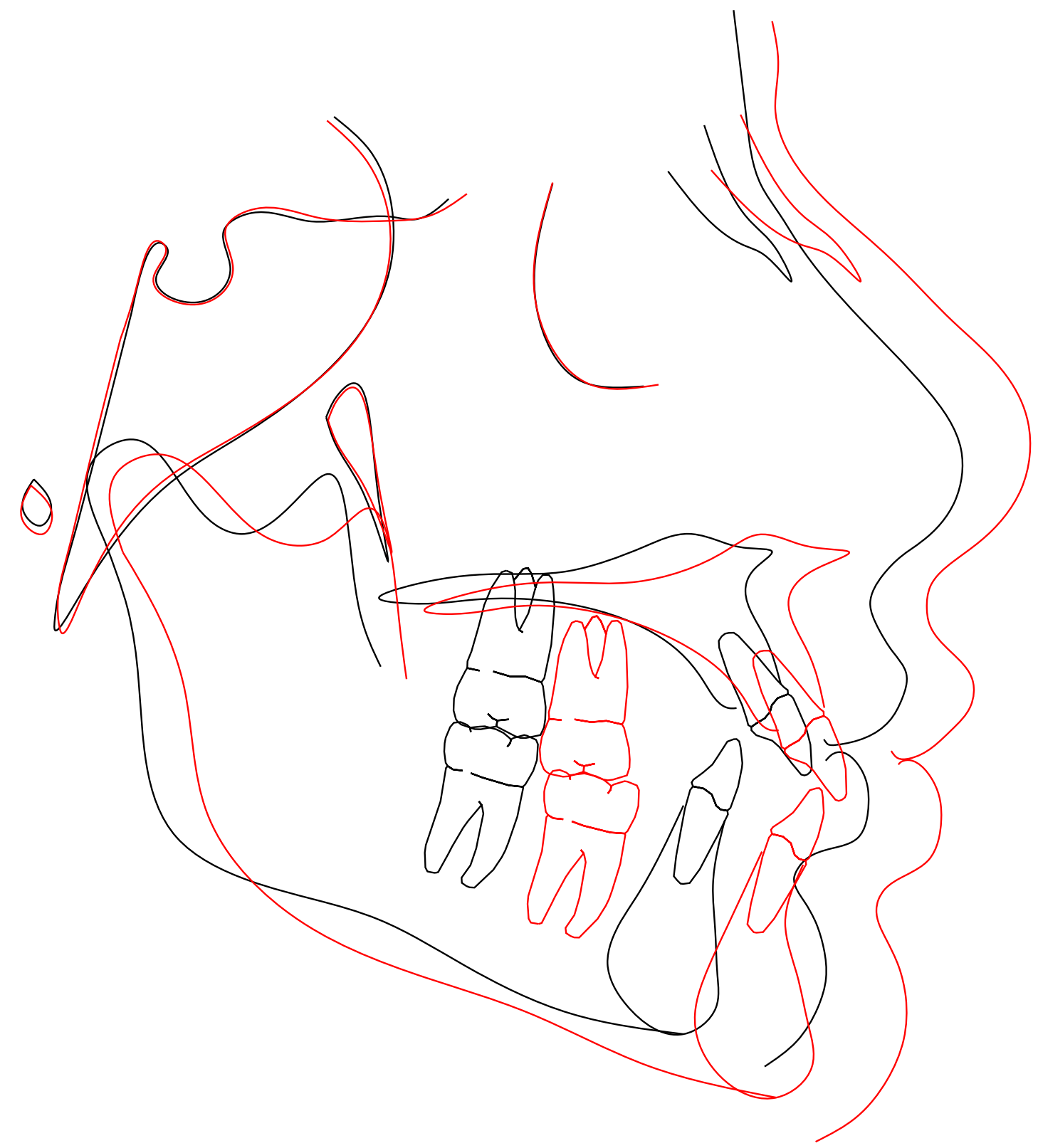

Figure F-48. Print of cephalometric tracing superimposition subject 48 (tracing is 1:1 with original radiograph). 


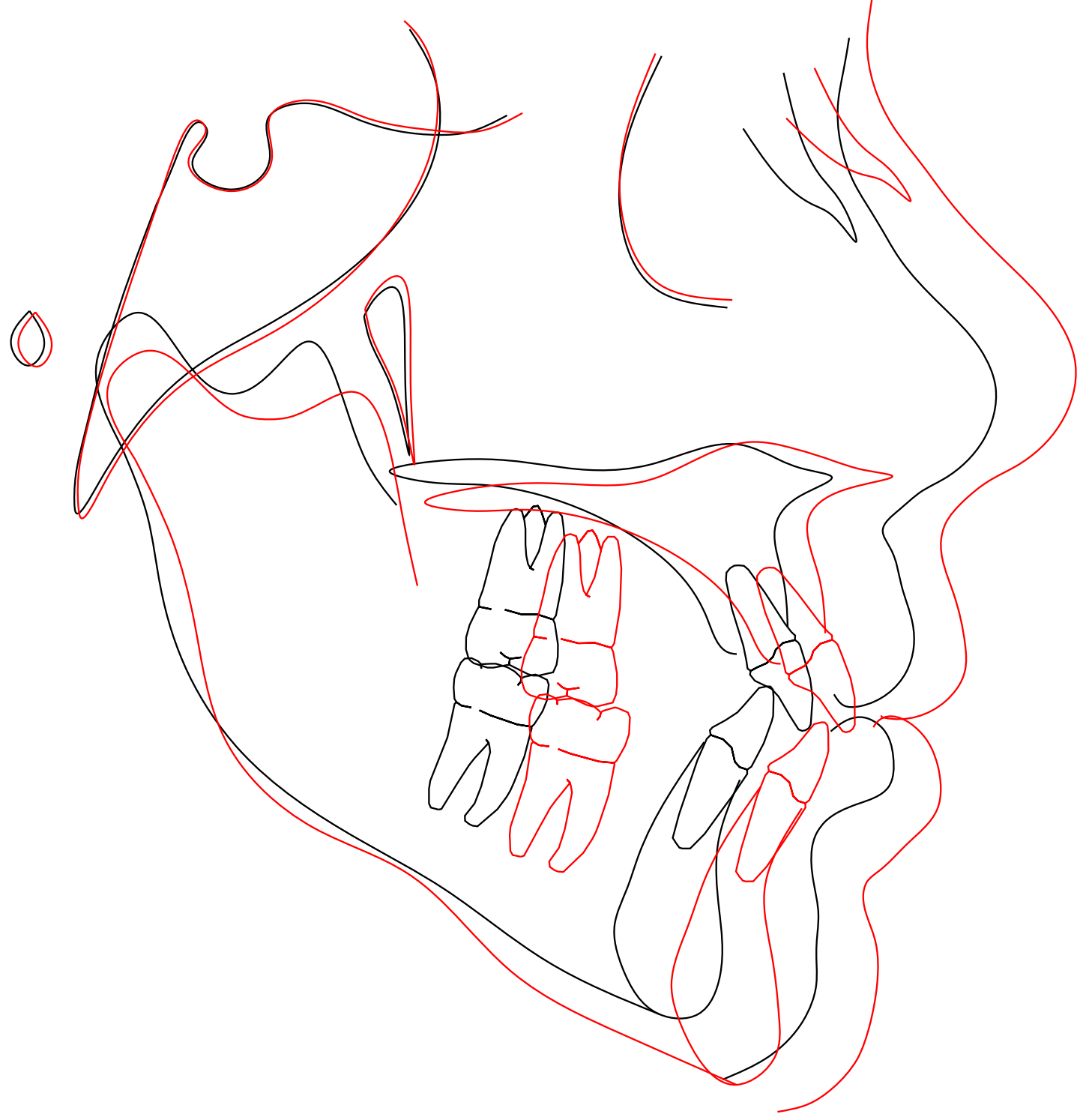

Figure F-49. Print of cephalometric tracing superimposition subject 49 (tracing is 1:1 with original radiograph). 


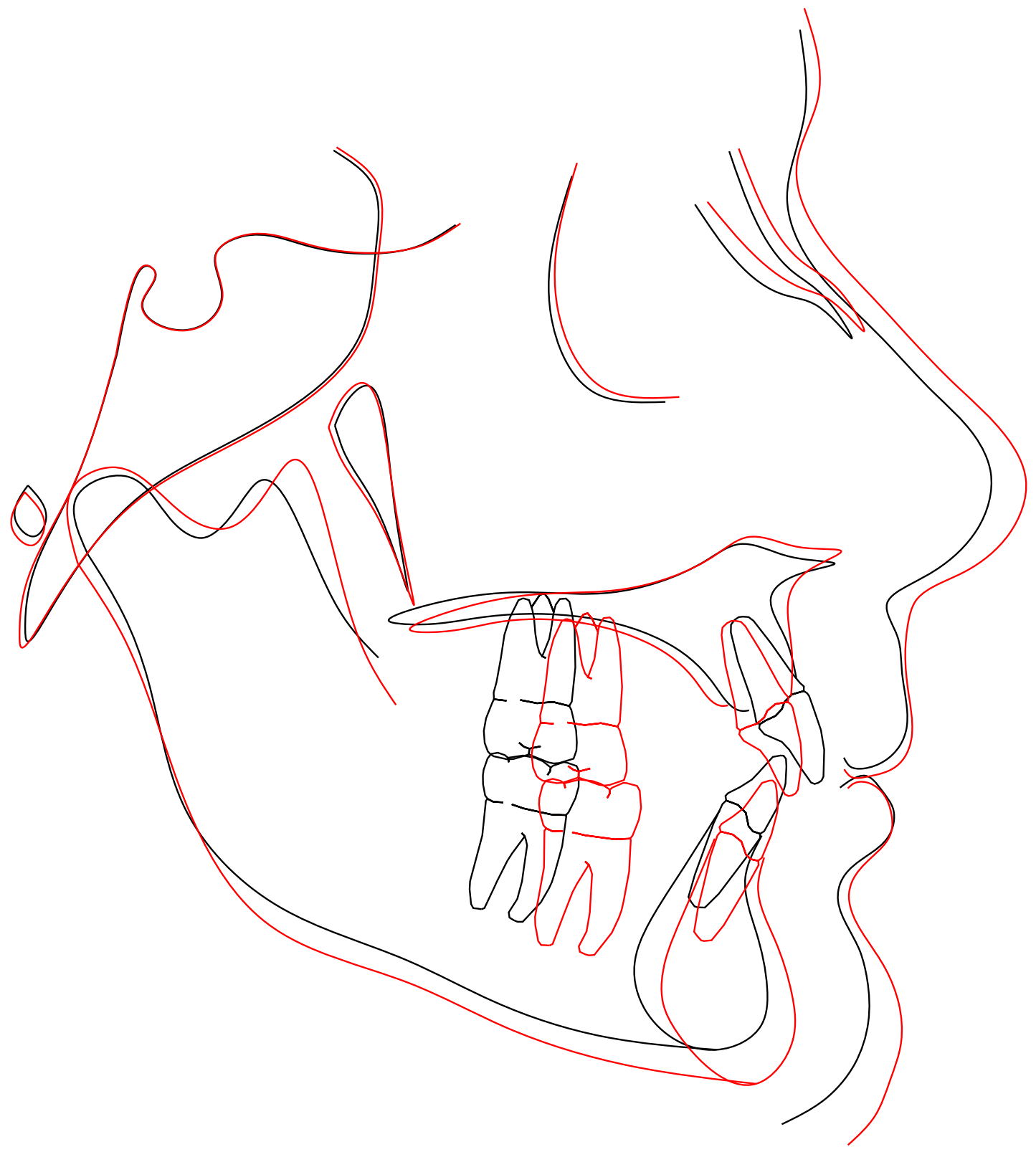

Figure F-50. Print of cephalometric tracing superimposition subject 50 (tracing is 1:1 with original radiograph). 


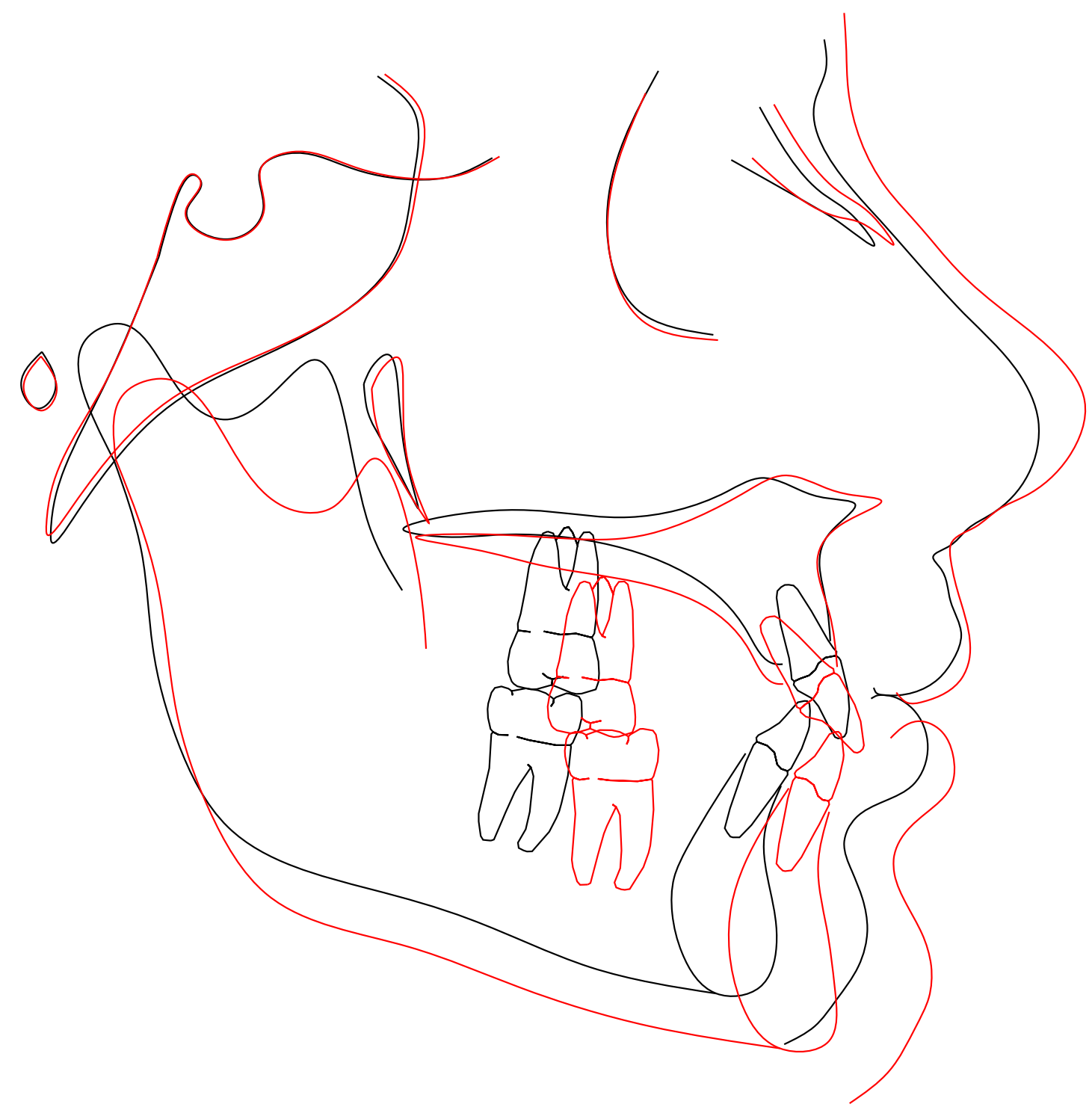

Figure F-51. Print of cephalometric tracing superimposition subject 51 (tracing is 1:1 with original radiograph). 


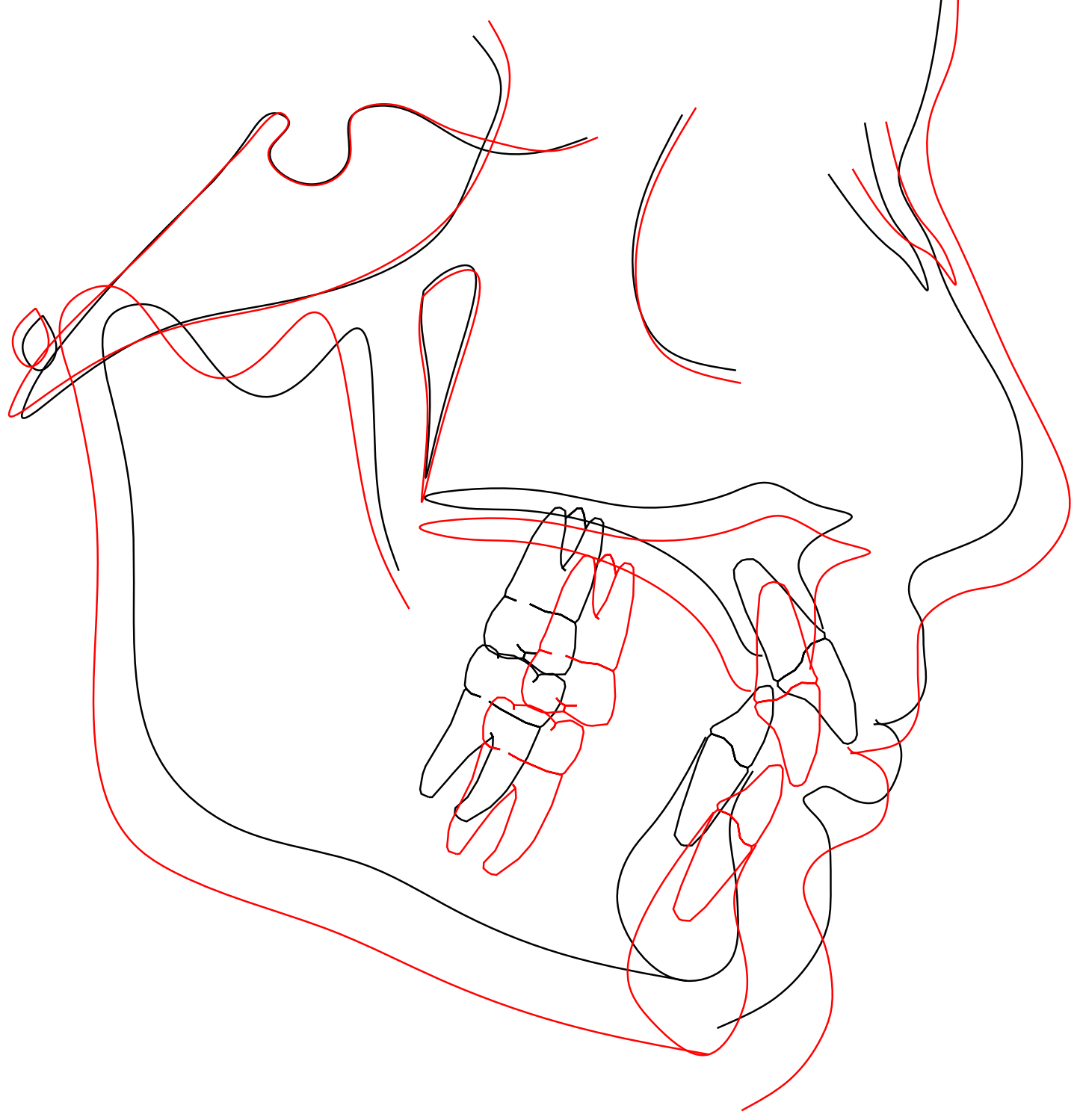

Figure F-52. Print of cephalometric tracing superimposition subject 52 (tracing is 1:1 with original radiograph). 


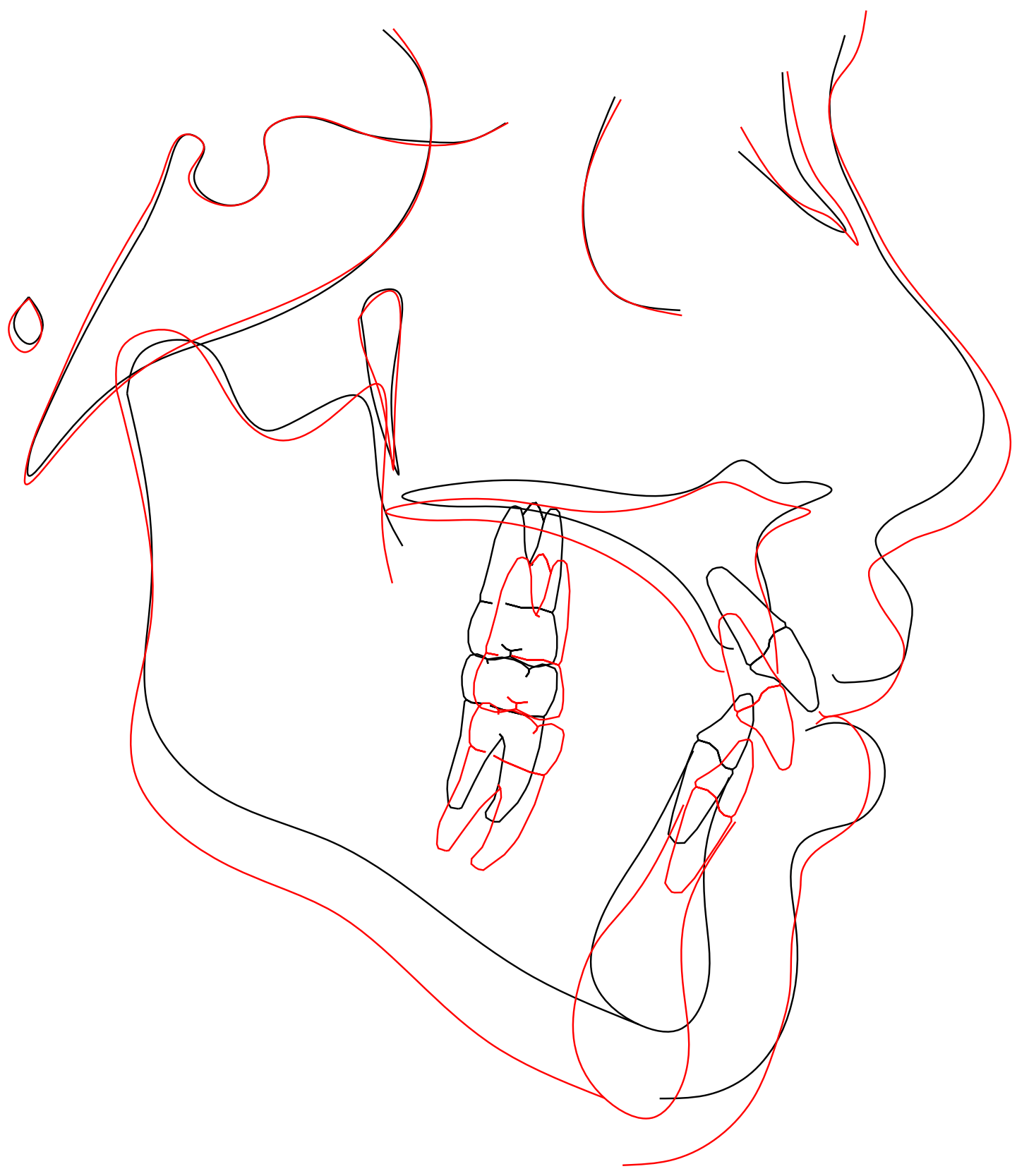

Figure F-53. Print of cephalometric tracing superimposition subject 53 (tracing is 1:1 with original radiograph). 


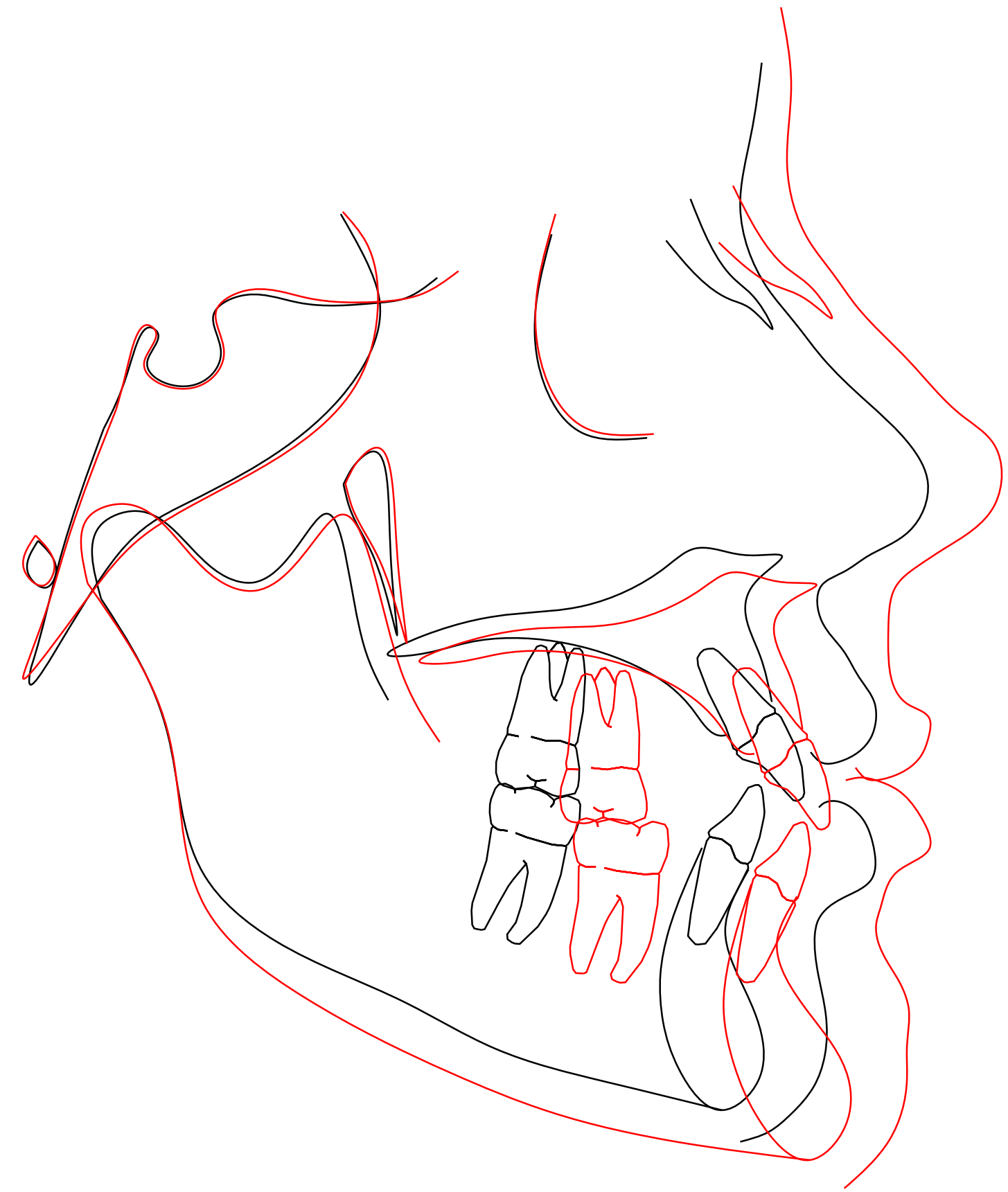

Figure F-54. Print of cephalometric tracing superimposition subject 54 (tracing is 1:1 with original radiograph). 


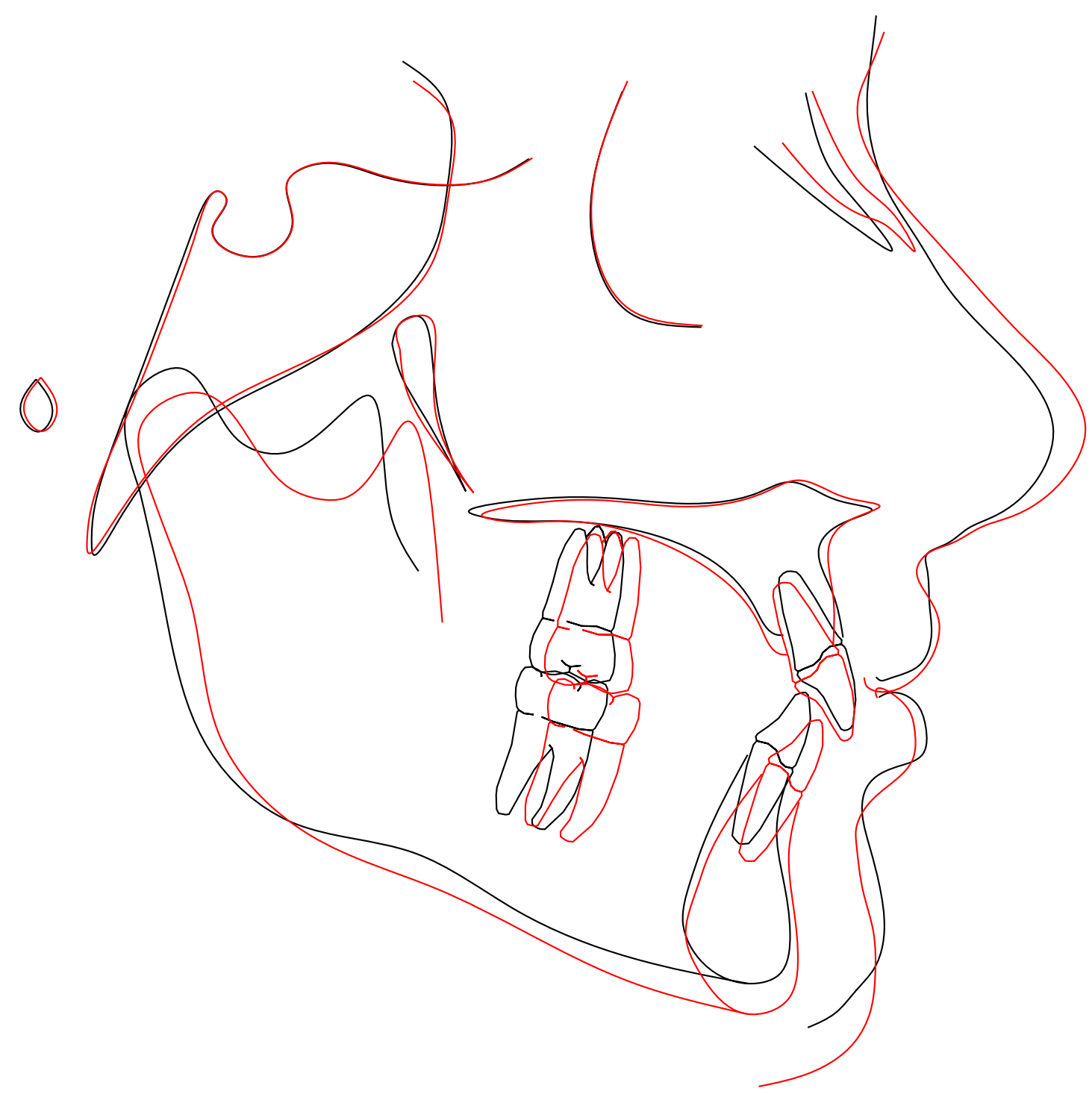

Figure F-55. Print of cephalometric tracing superimposition subject 55 (tracing is 1:1 with original radiograph). 


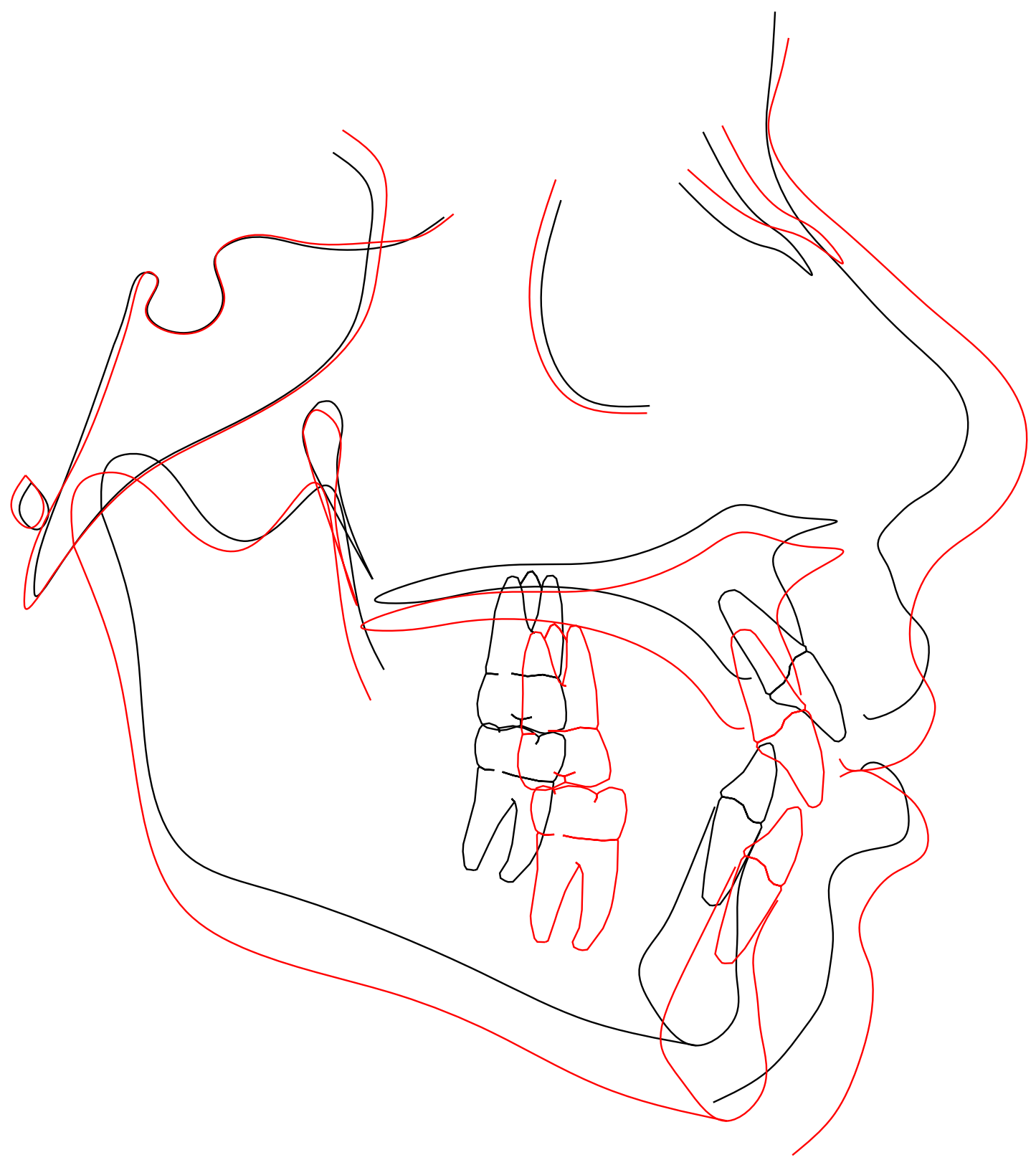

Figure F-56. Print of cephalometric tracing superimposition subject 56 (tracing is 1:1 with original radiograph). 
0

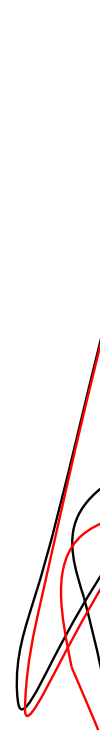

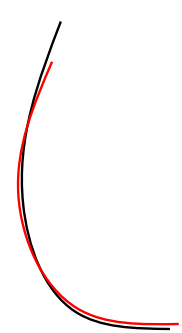

/
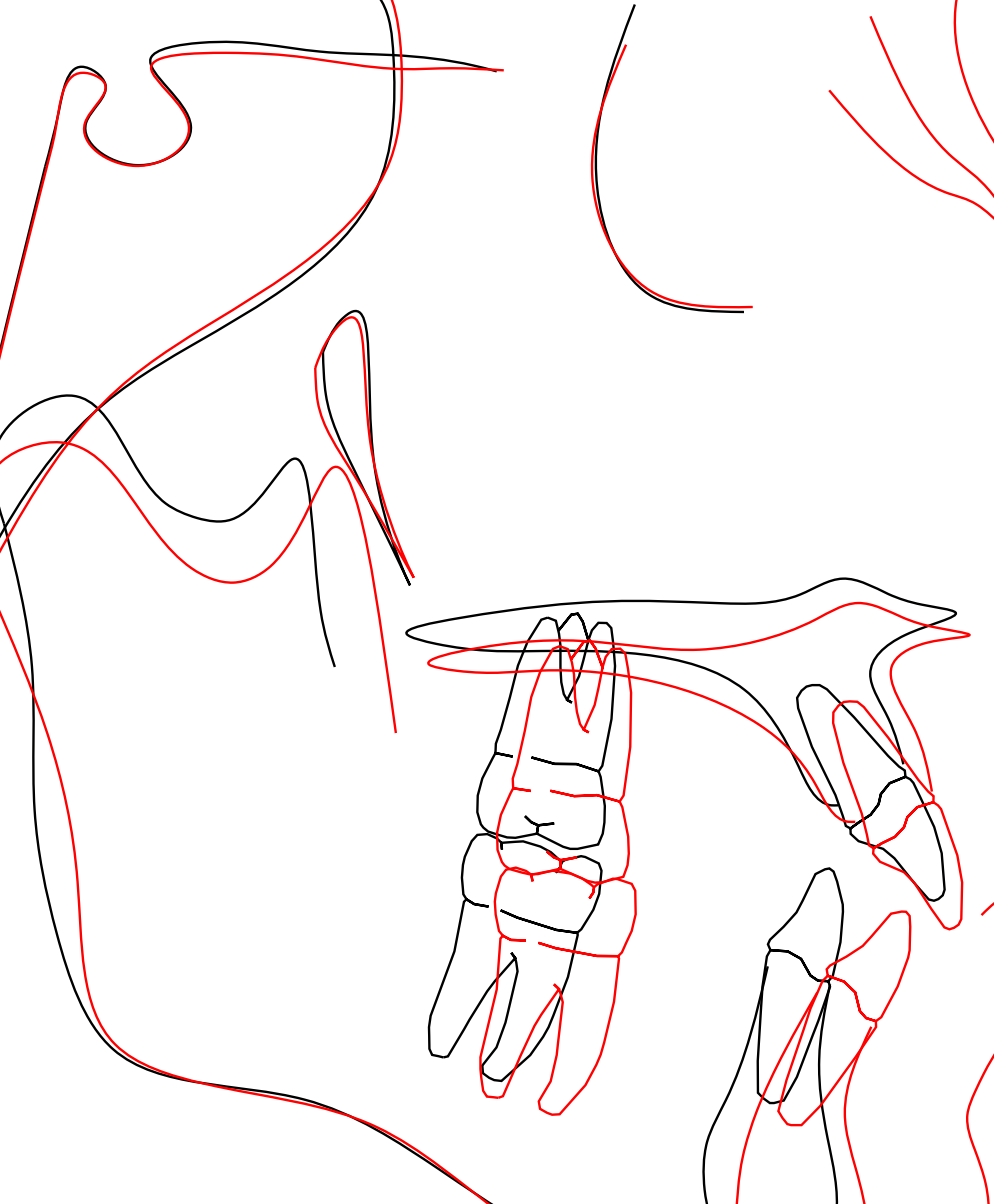

w

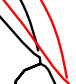

.
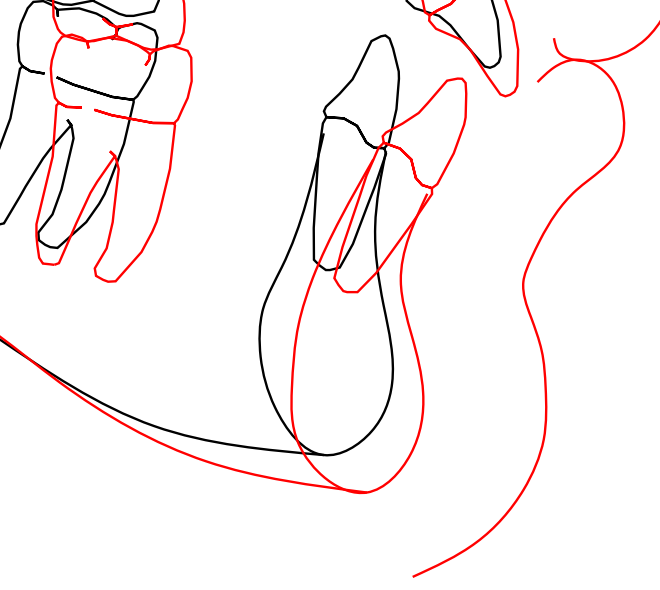

Figure F-57. Print of cephalometric tracing superimposition subject 57 (tracing is 1:1 with original radiograph). 


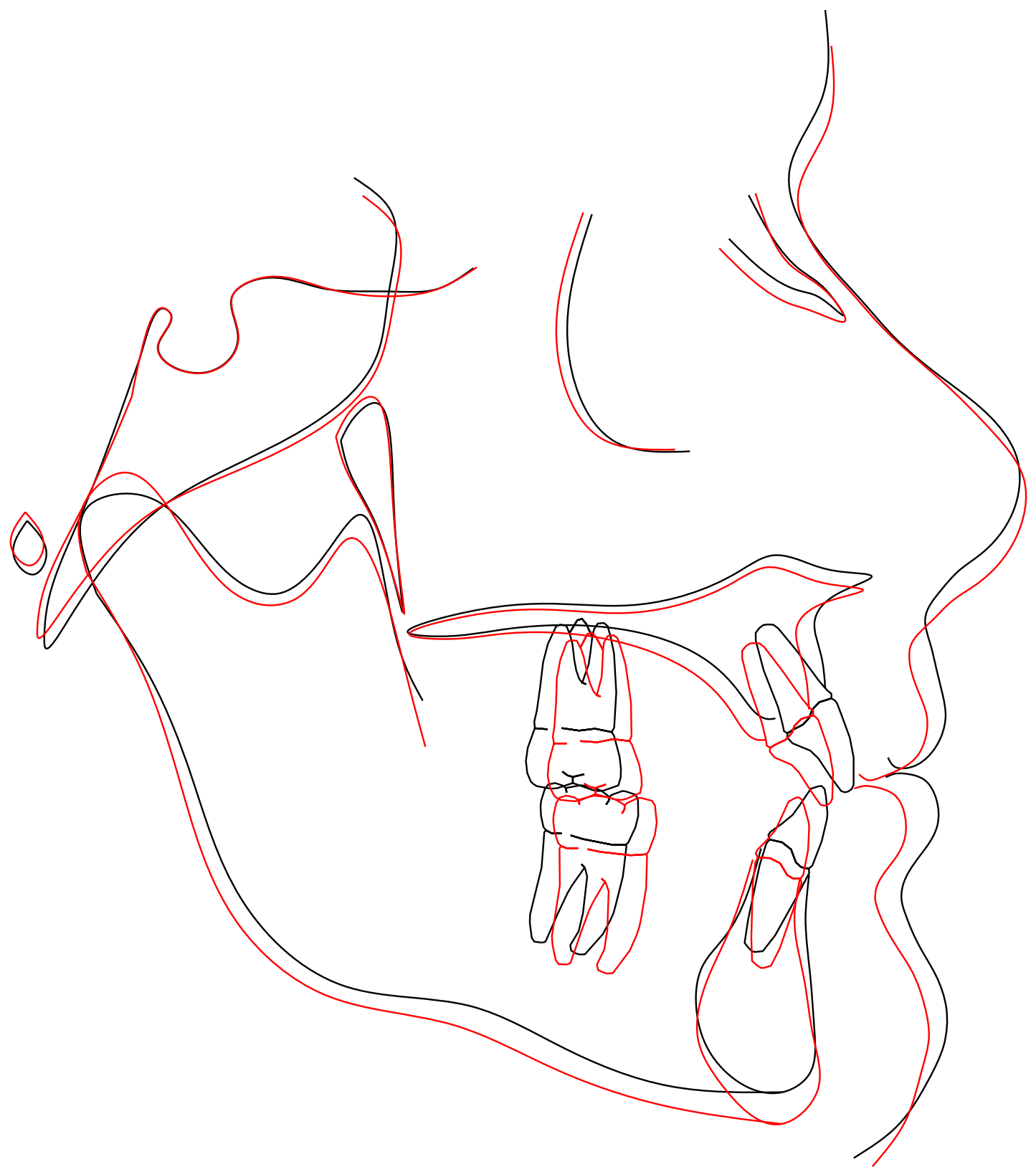

Figure F-58. Print of cephalometric tracing superimposition subject 58 (tracing is 1:1 with original radiograph). 


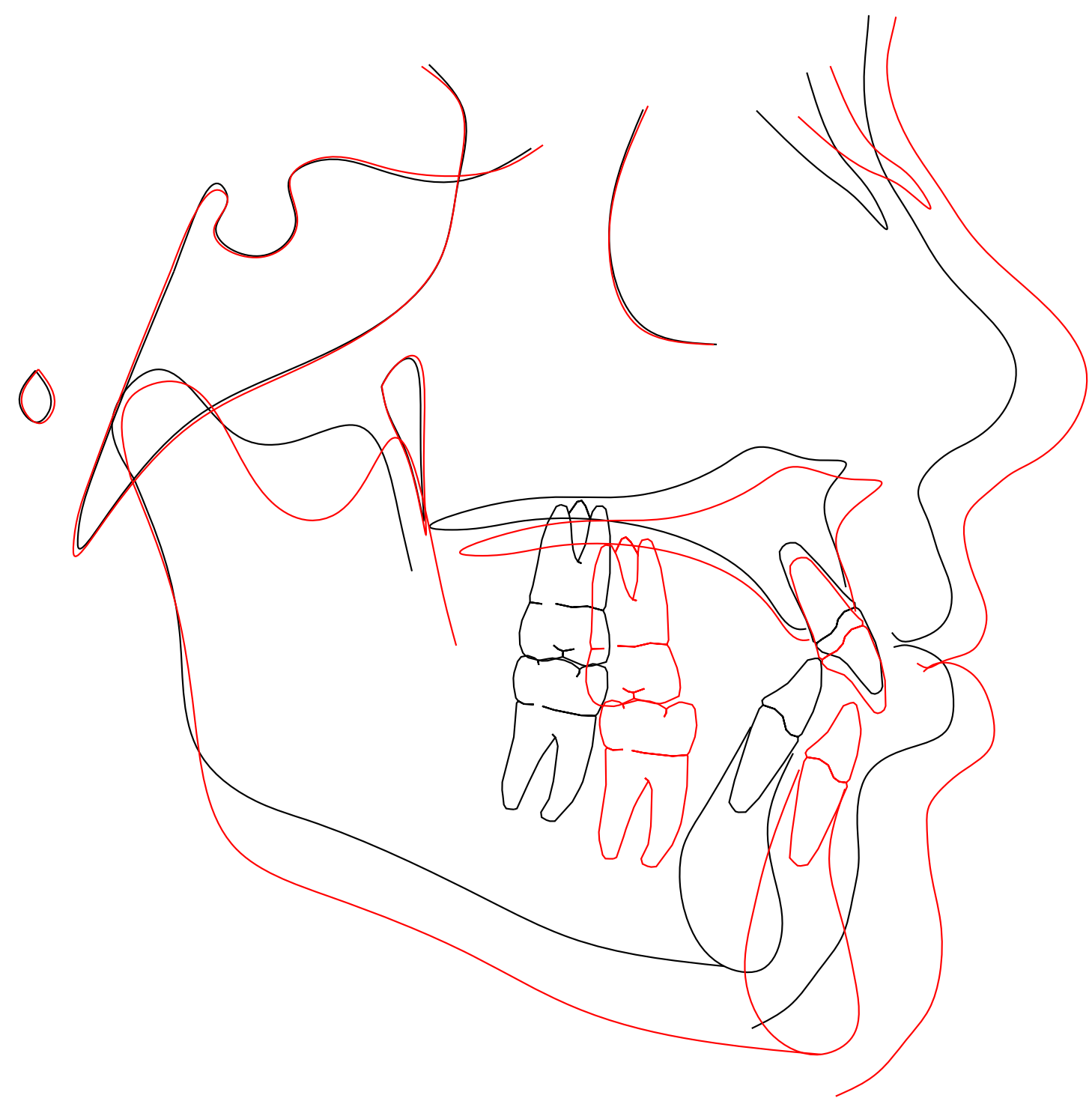

Figure F-59. Print of cephalometric tracing superimposition subject 59 (tracing is 1:1 with original radiograph). 


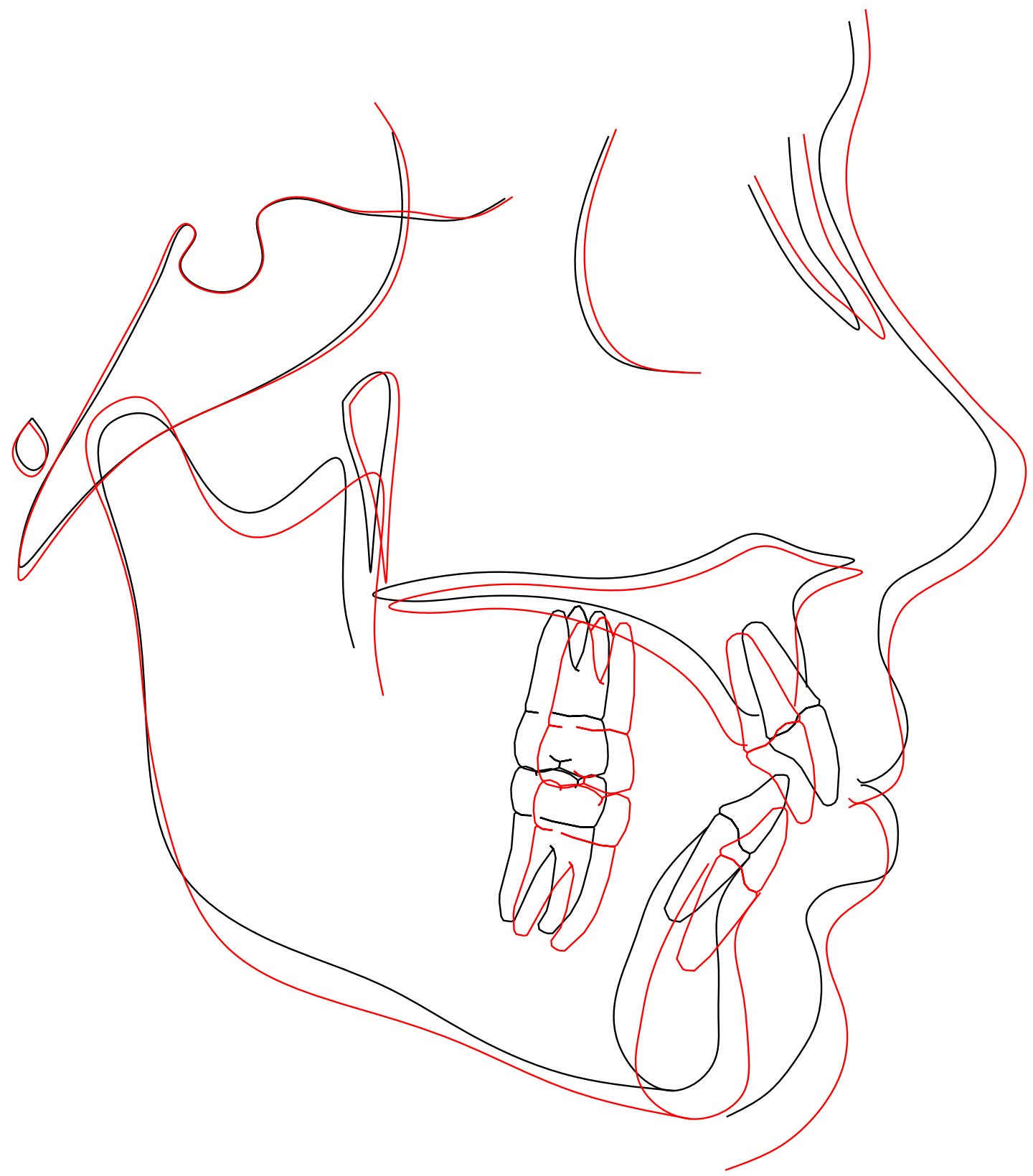

Figure F-60. Print of cephalometric tracing superimposition subject 60 (tracing is 1:1 with original radiograph). 


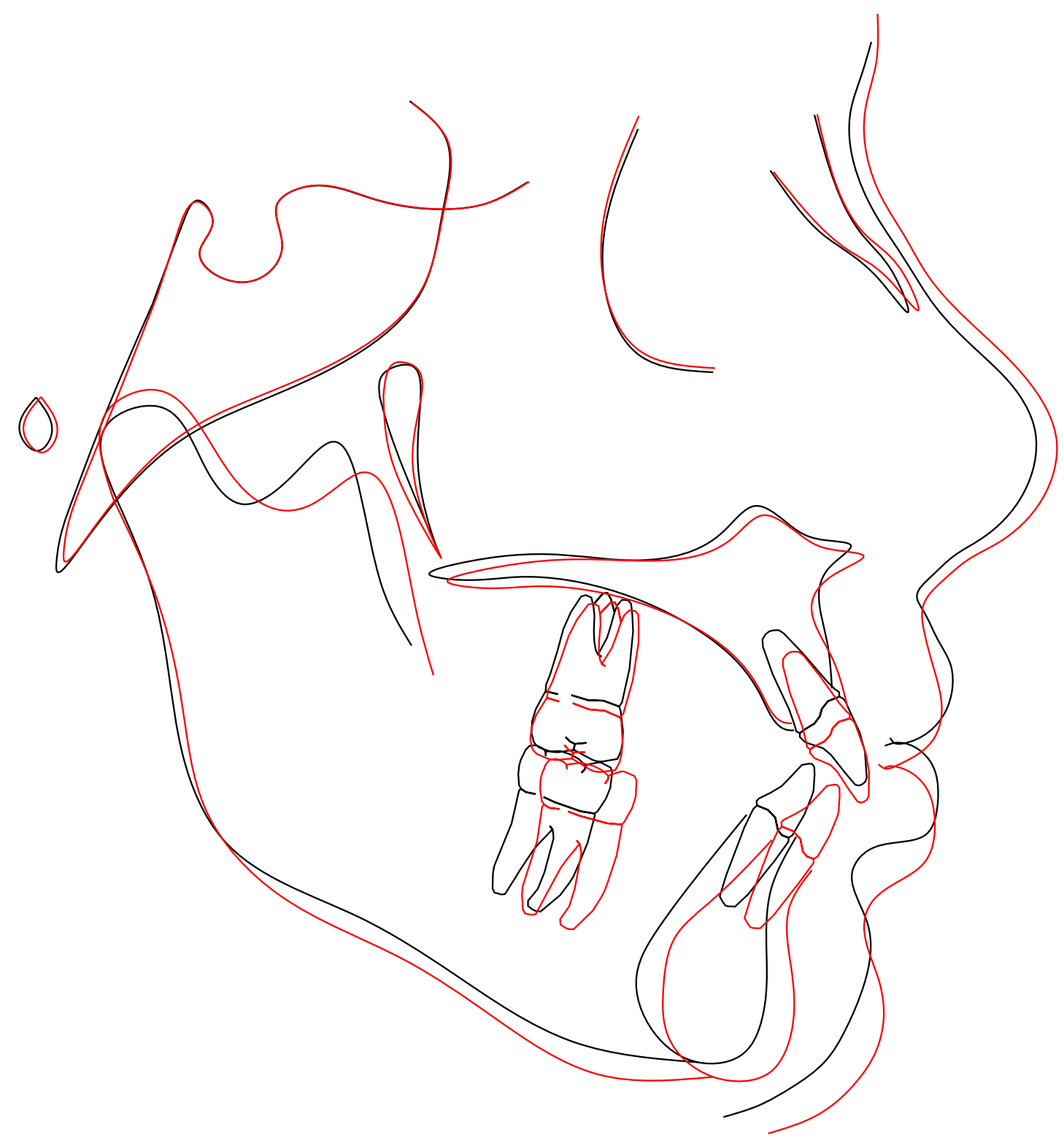

Figure F-61. Print of cephalometric tracing superimposition subject 61 (tracing is 1:1 with original radiograph). 


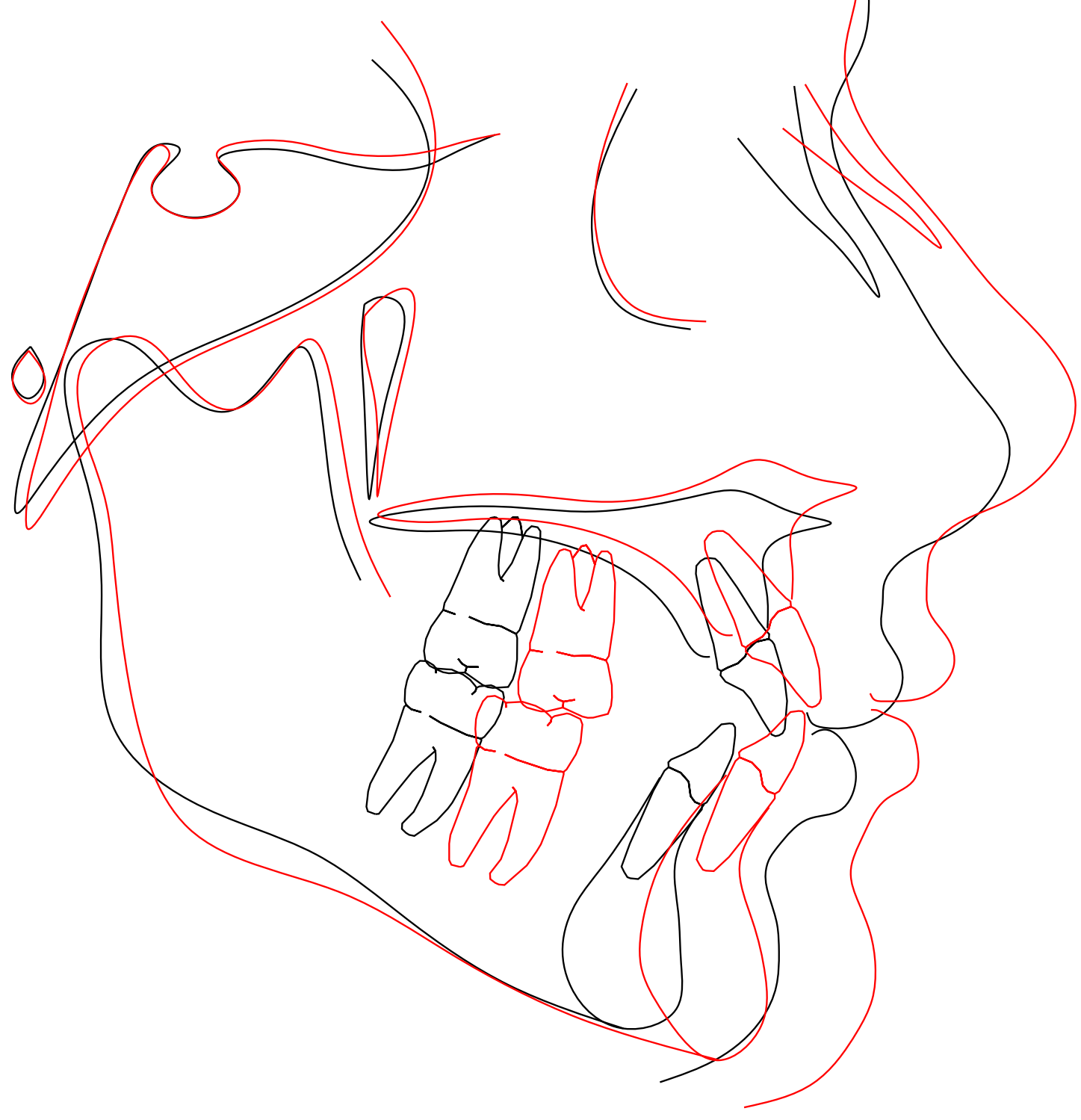

Figure F-62. Print of cephalometric tracing superimposition subject 62 (tracing is 1:1 with original radiograph). 


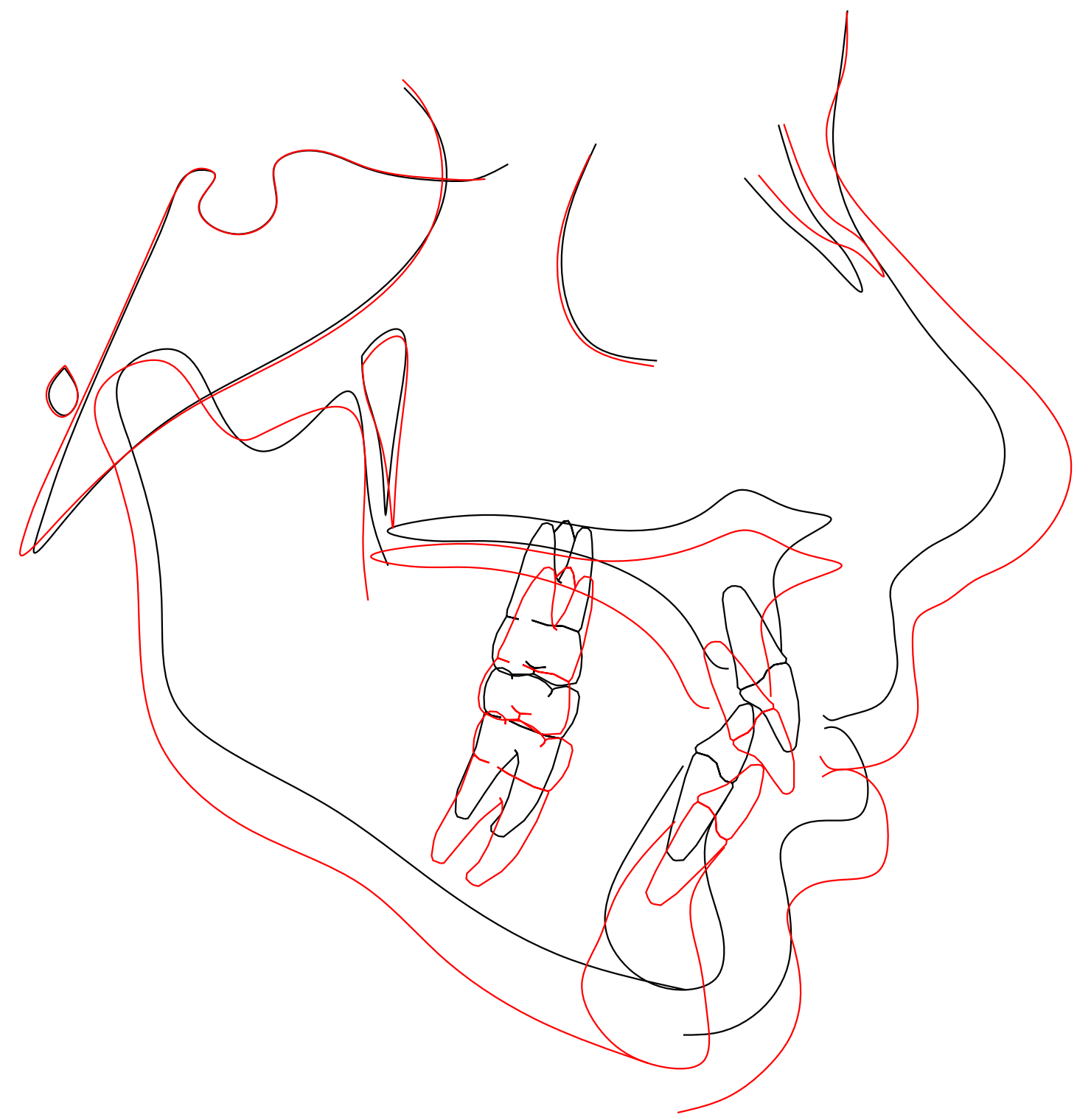

Figure F-63. Print of cephalometric tracing superimposition subject 63 (tracing is 1:1 with original radiograph). 


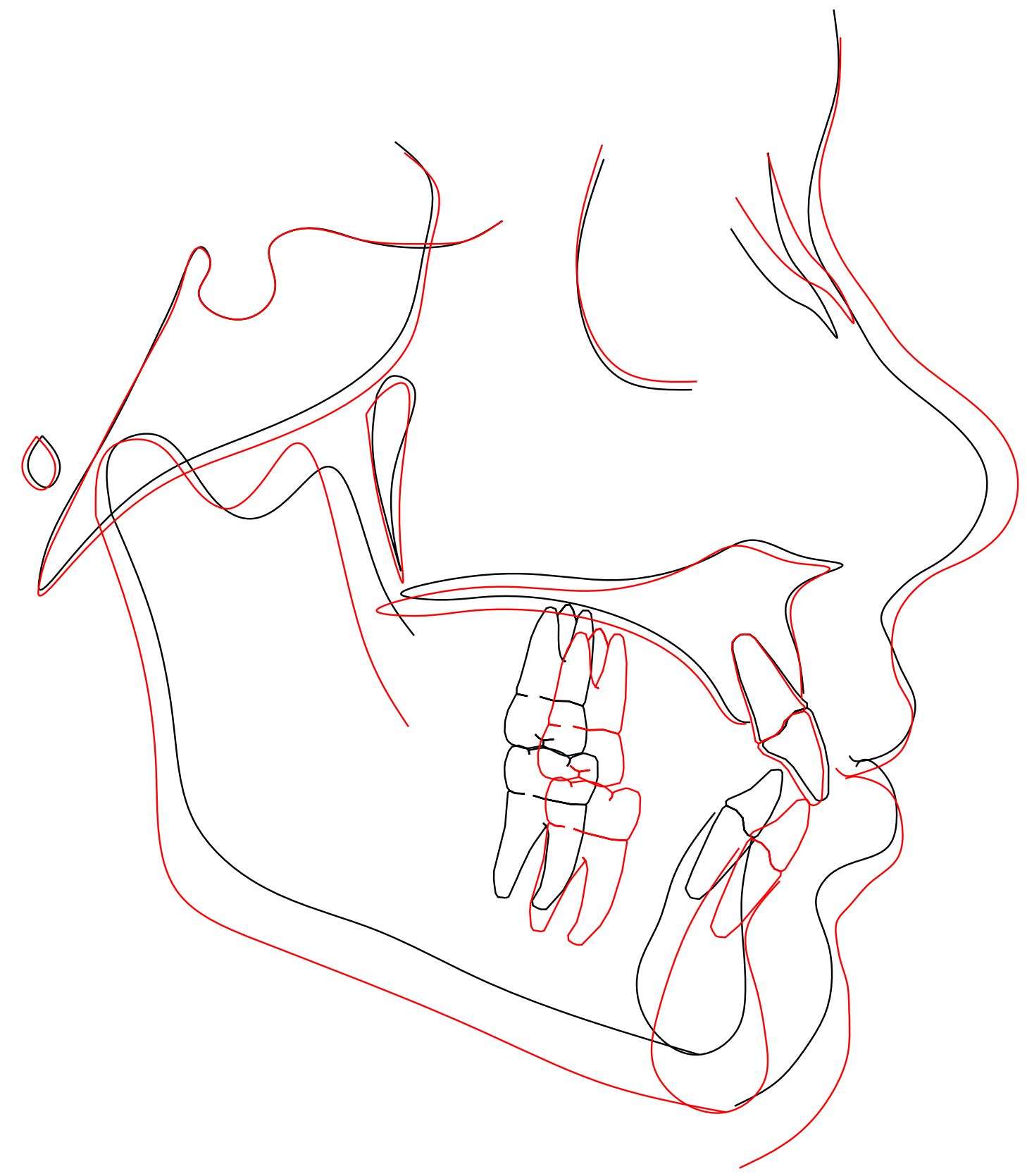

Figure F-64. Print of cephalometric tracing superimposition subject 64 (tracing is 1:1 with original radiograph). 


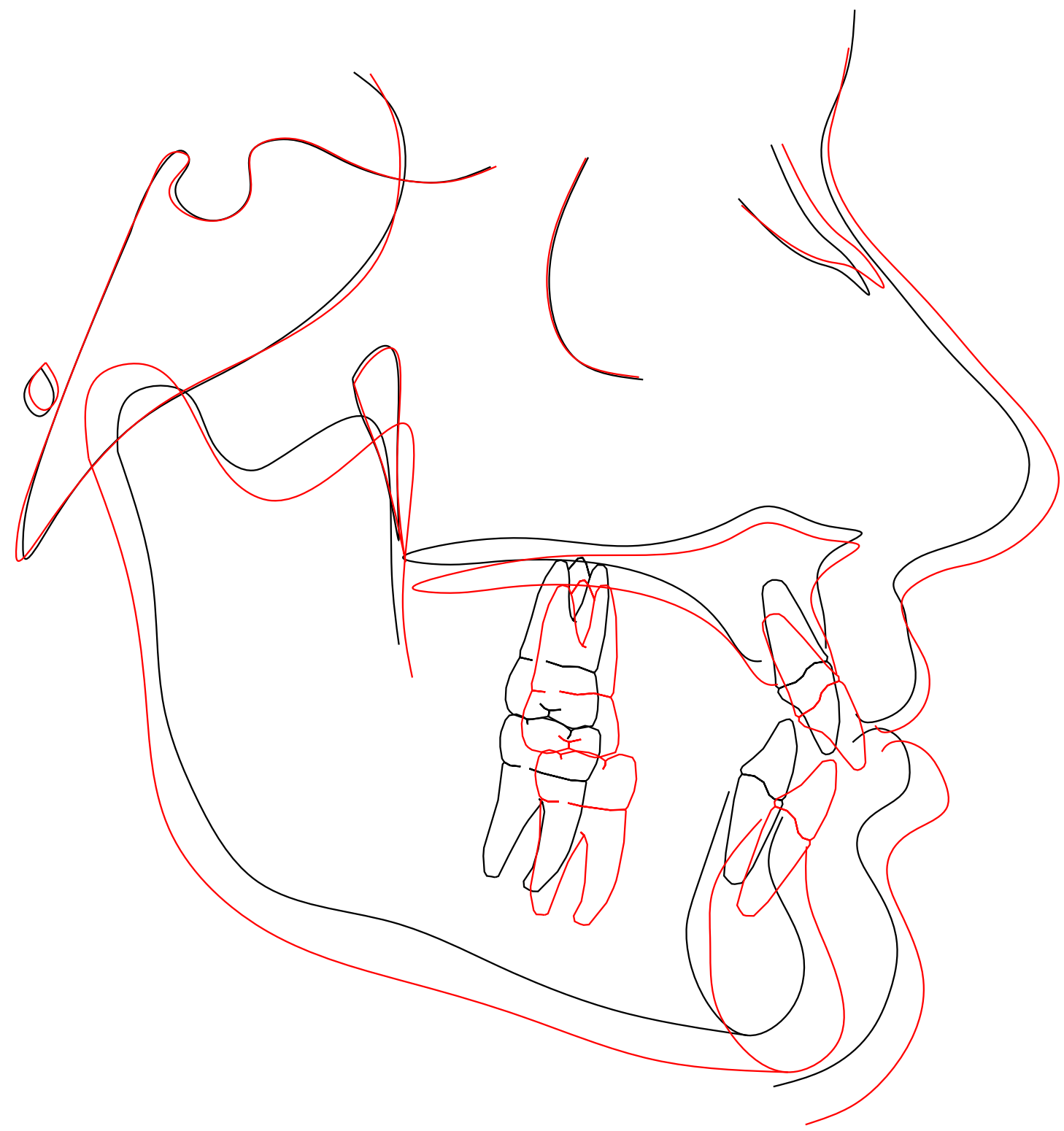

Figure F-65. Print of cephalometric tracing superimposition subject 65 (tracing is 1:1 with original radiograph). 


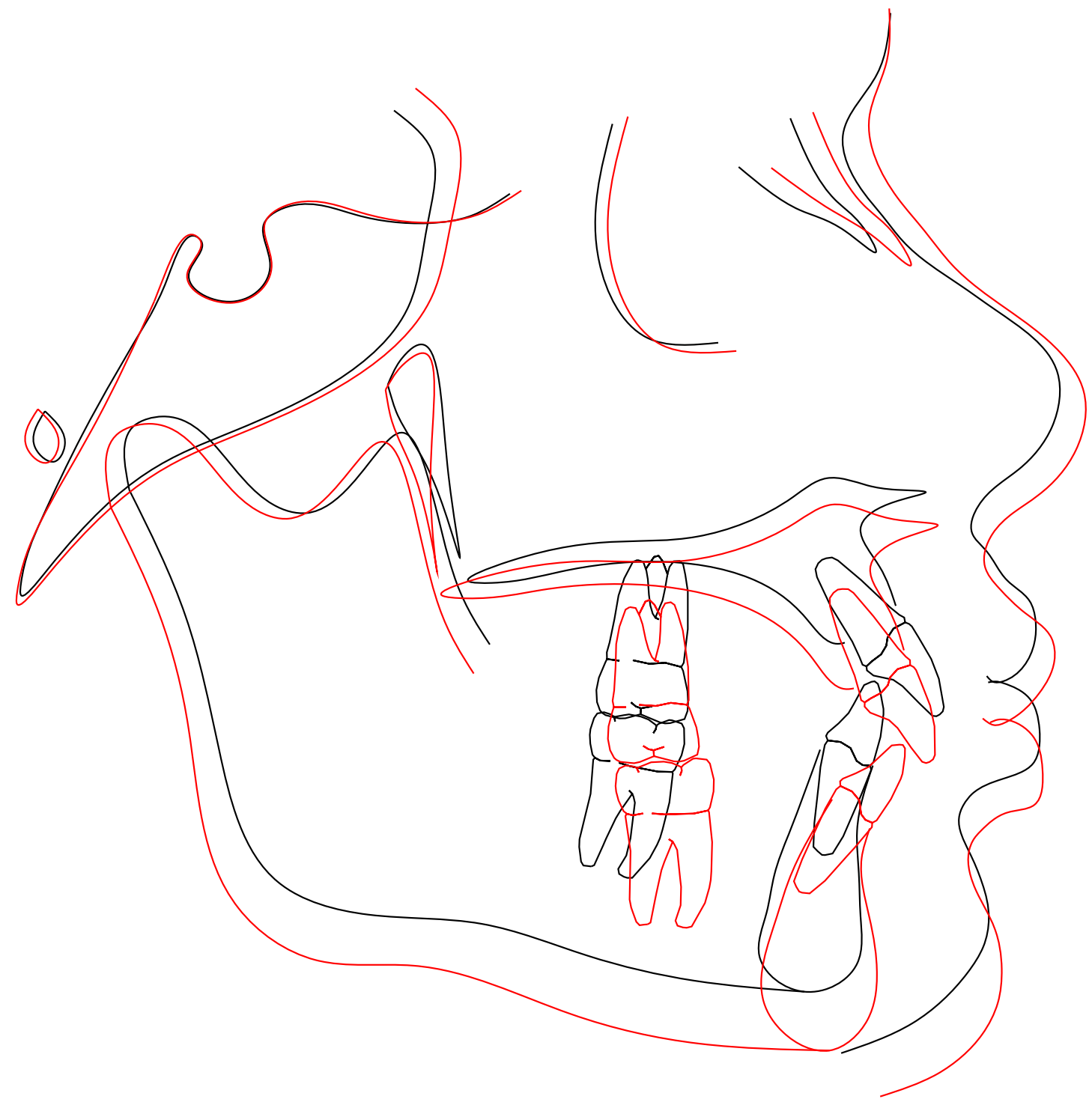

Figure F-66. Print of cephalometric tracing superimposition subject 66 (tracing is 1:1 with original radiograph). 


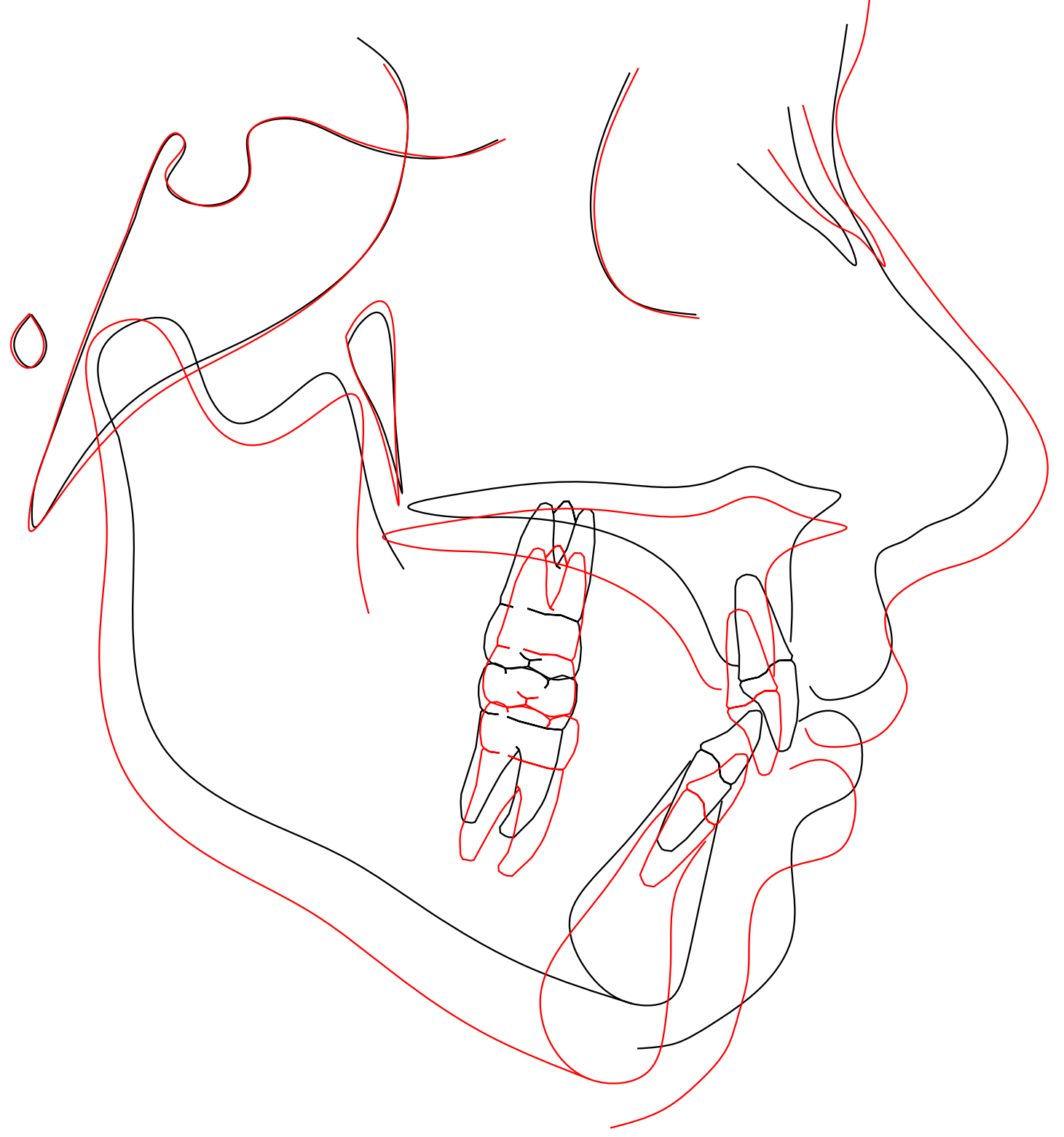

Figure F-67. Print of cephalometric tracing superimposition subject 67 (tracing is 1:1 with original radiograph). 


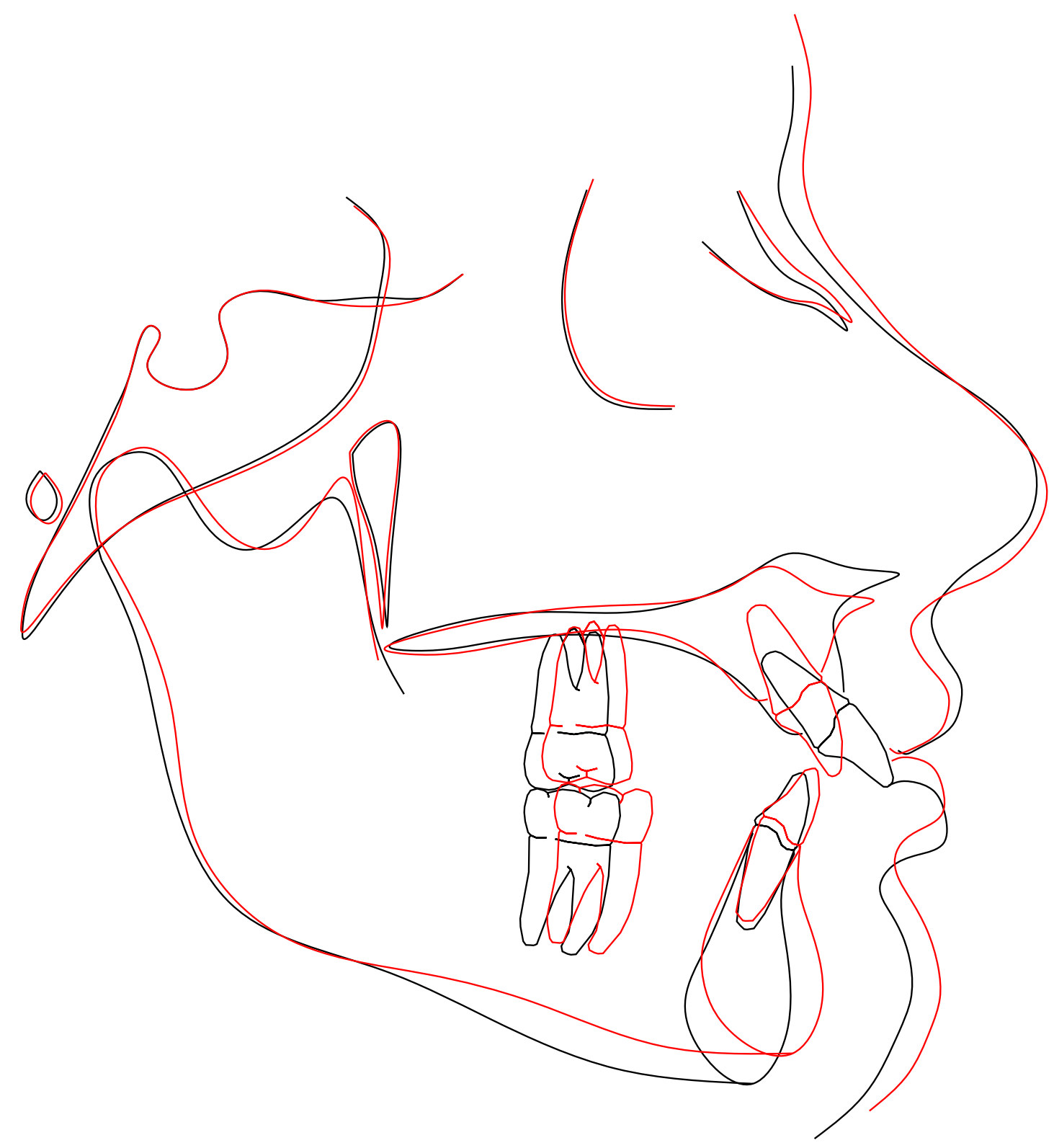

Figure F-68. Print of cephalometric tracing superimposition subject 68 (tracing is 1:1 with original radiograph). 


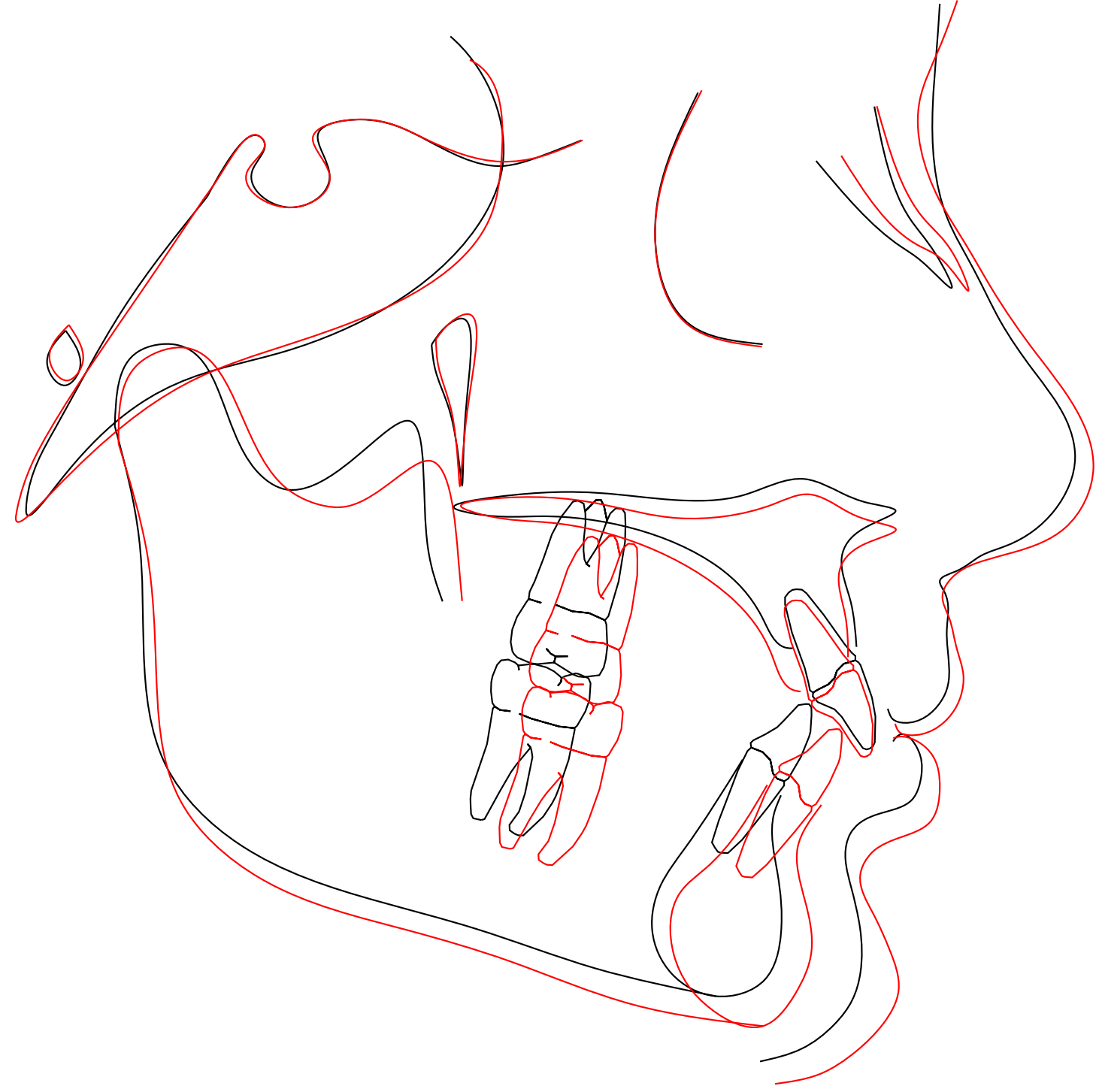

Figure F-69. Print of cephalometric tracing superimposition subject 69 (tracing is 1:1 with original radiograph). 


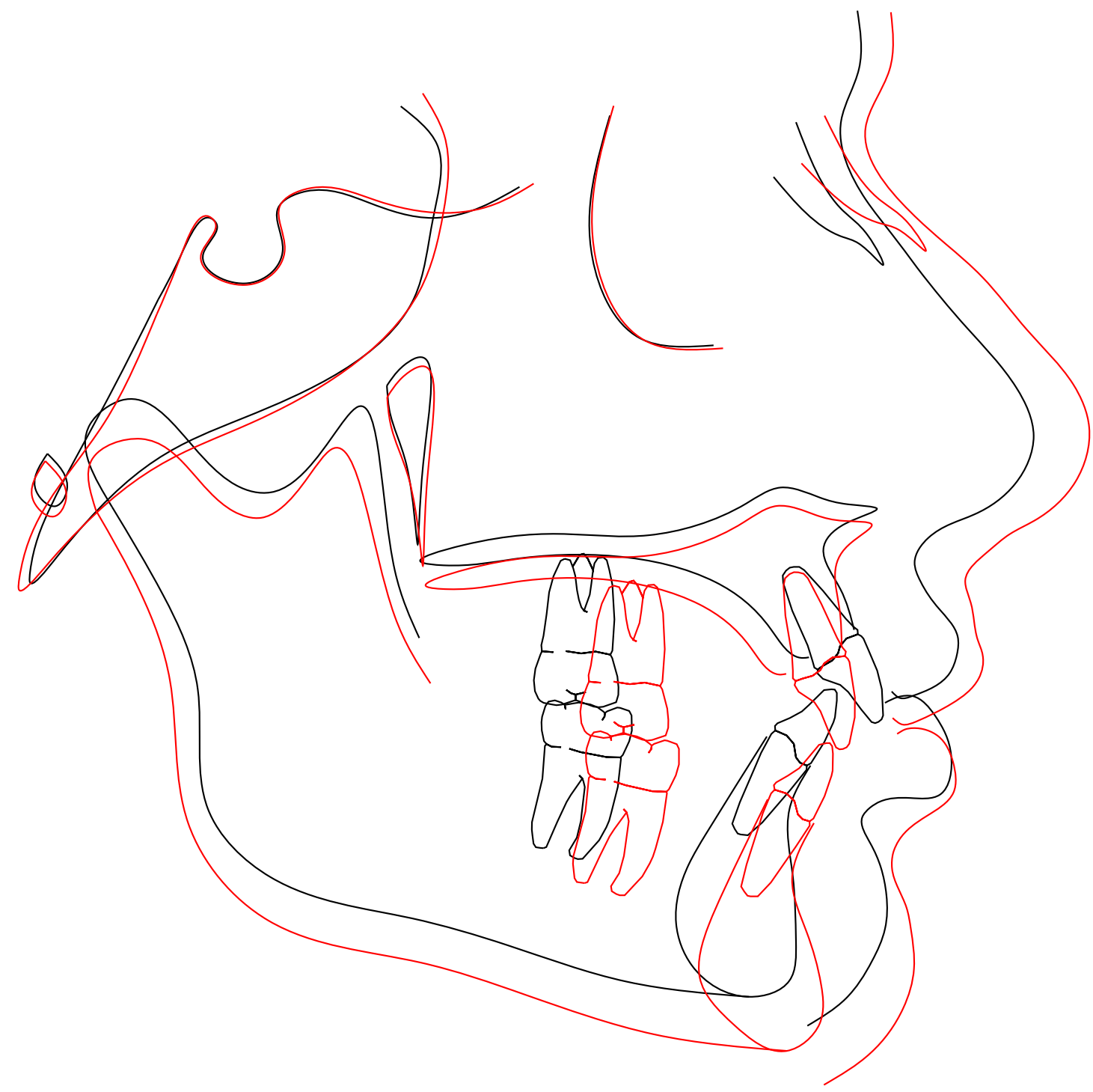

Figure F-70. Print of cephalometric tracing superimposition subject 70 (tracing is 1:1 with original radiograph). 


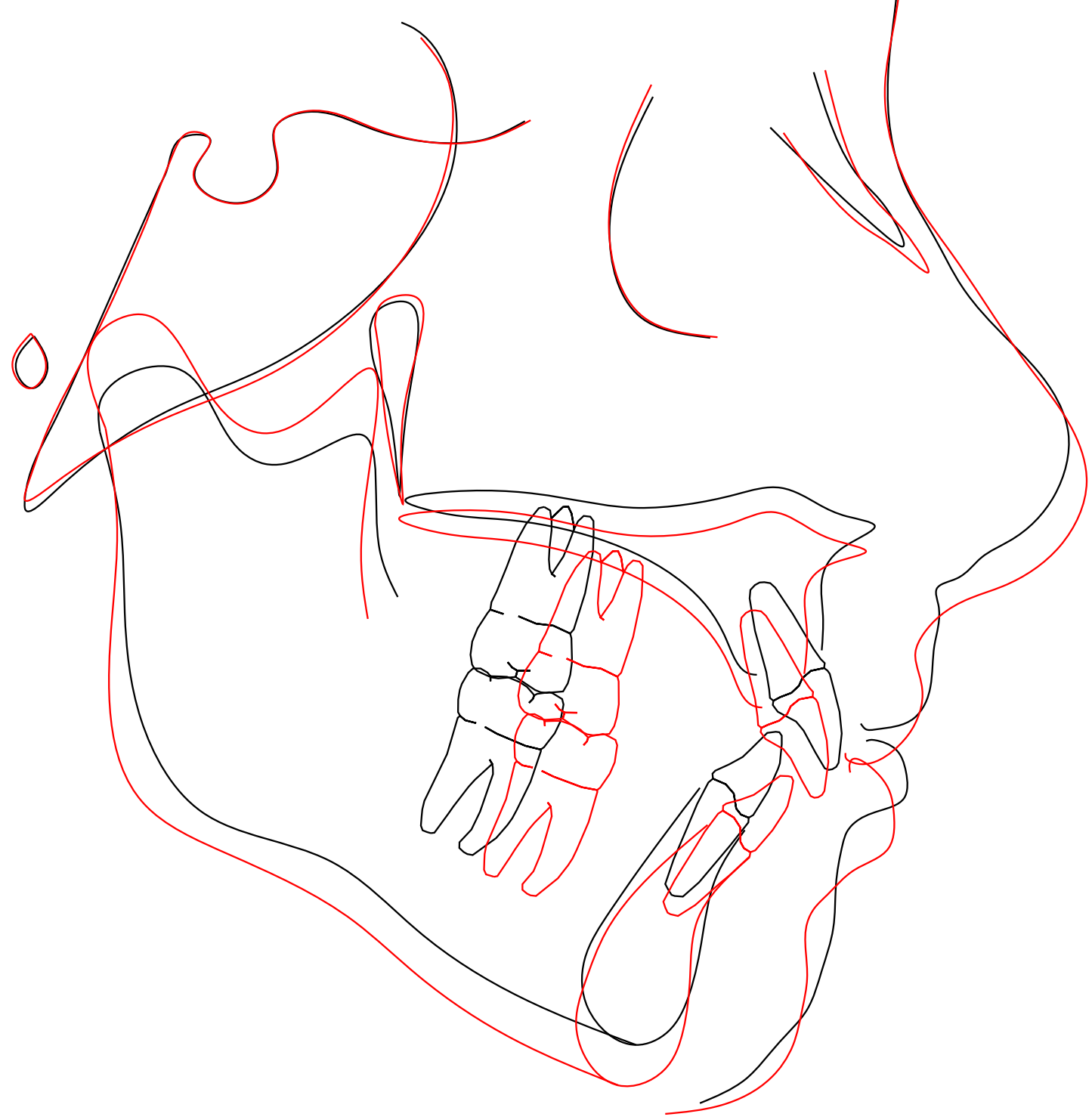

Figure F-71. Print of cephalometric tracing superimposition subject 71 (tracing is 1:1 with original radiograph). 


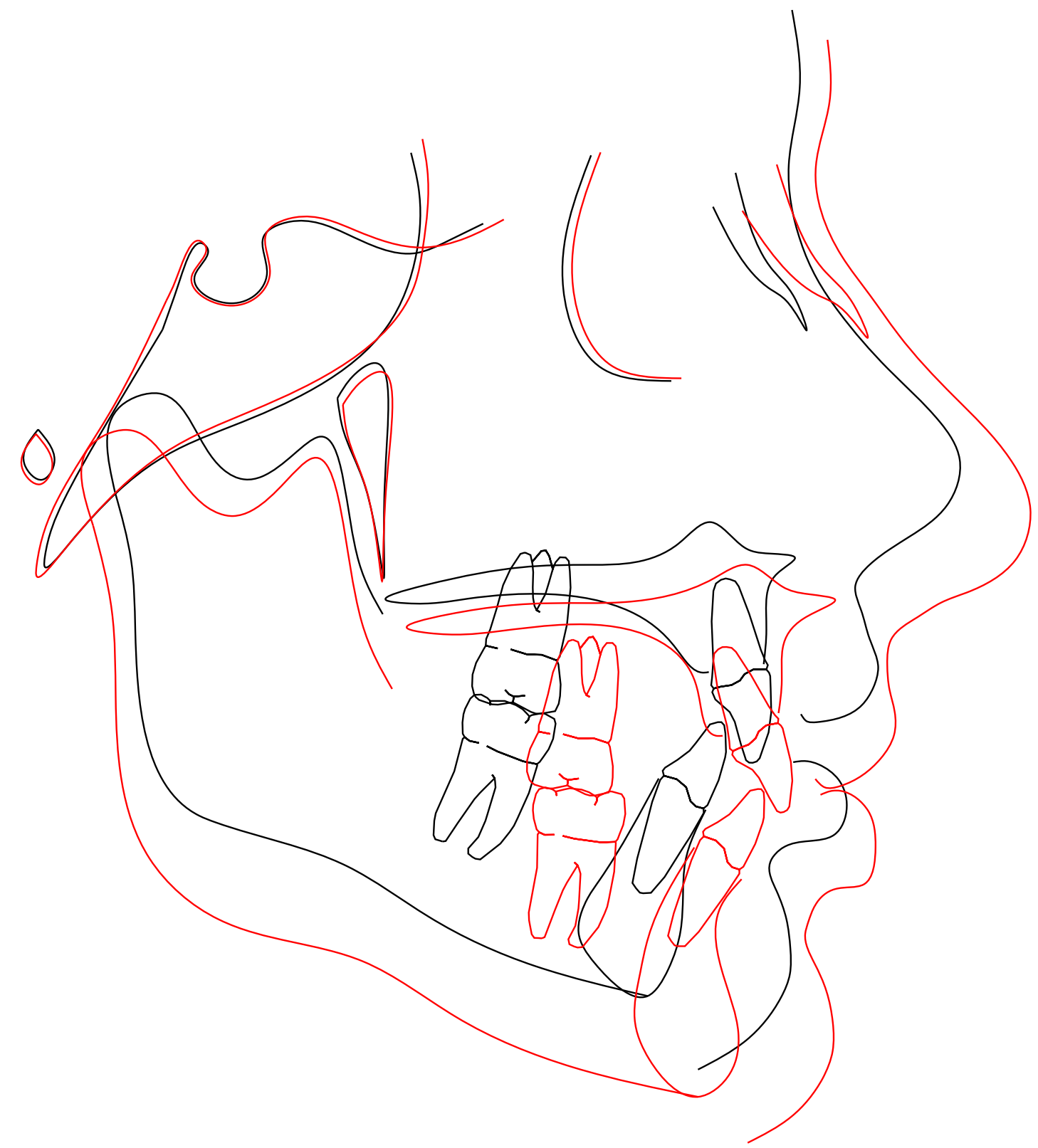

Figure F-72. Print of cephalometric tracing superimposition subject 72 (tracing is 1:1 with original radiograph). 


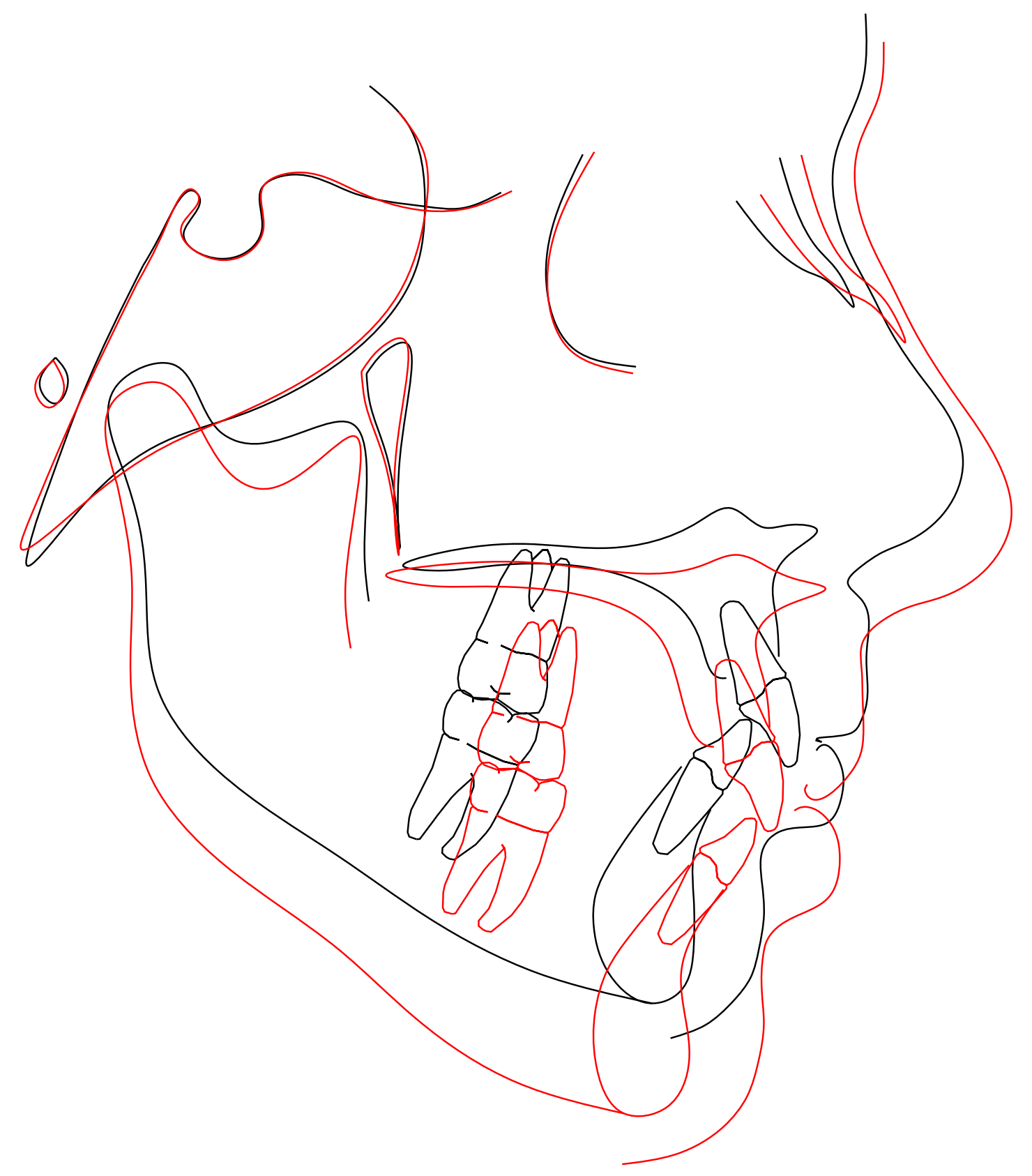

Figure F-73. Print of cephalometric tracing superimposition subject 73 (tracing is 1:1 with original radiograph). 


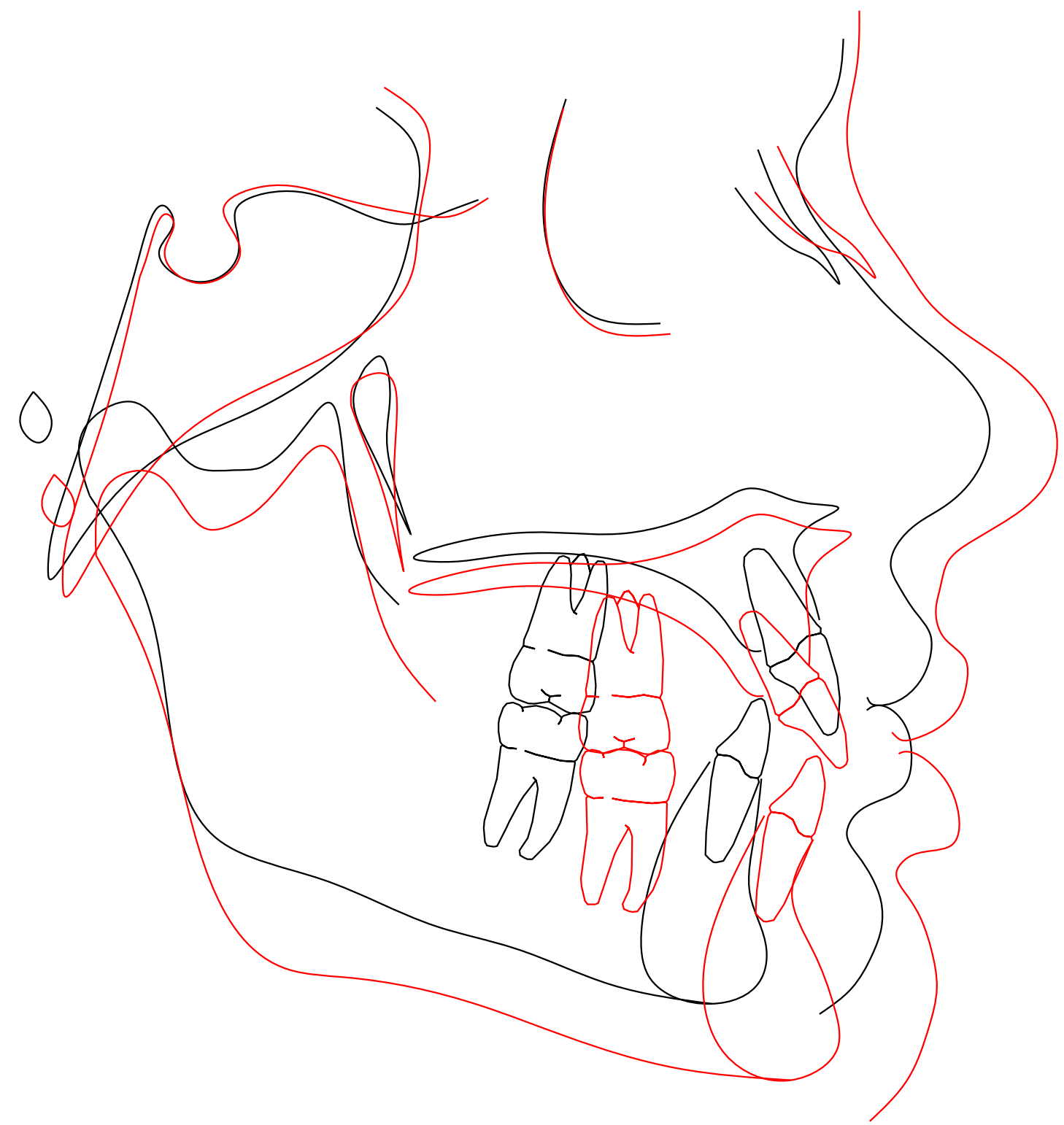

Figure F-74. Print of cephalometric tracing superimposition subject 74 (tracing is 1:1 with original radiograph). 


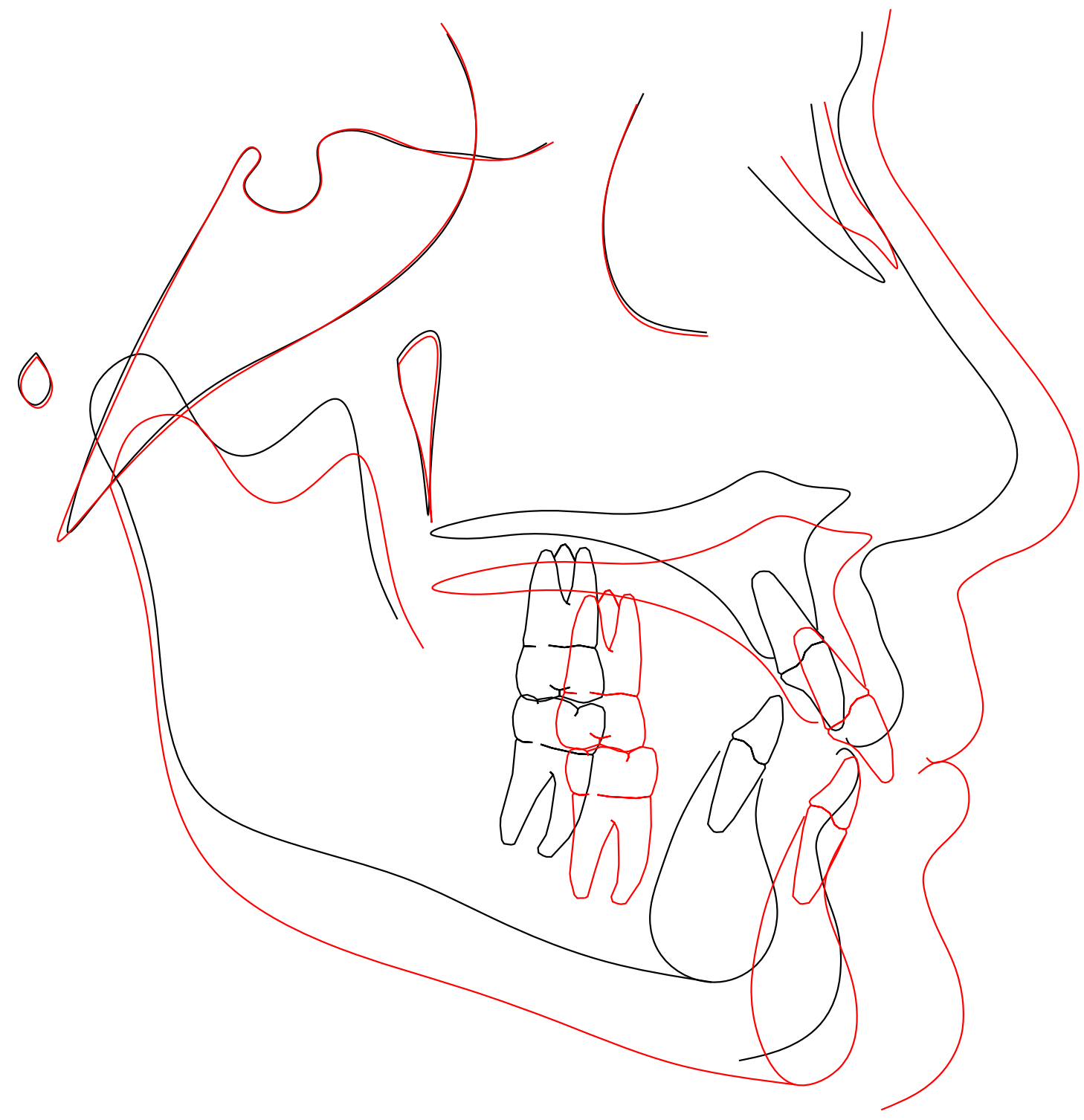

Figure F-75. Print of cephalometric tracing superimposition subject 75 (tracing is 1:1 with original radiograph). 


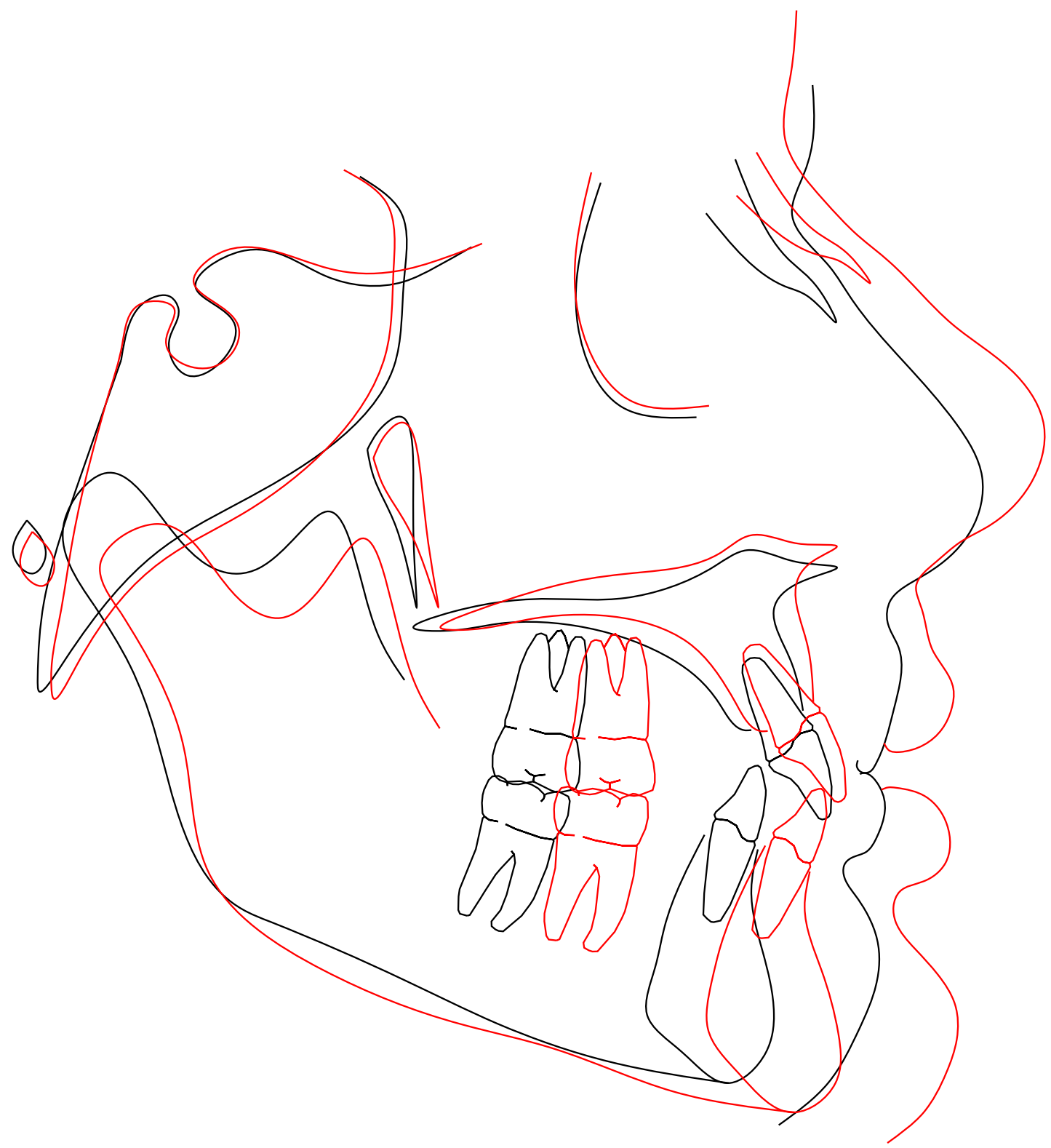

Figure F-76. Print of cephalometric tracing superimposition subject 76 (tracing is 1:1 with original radiograph). 


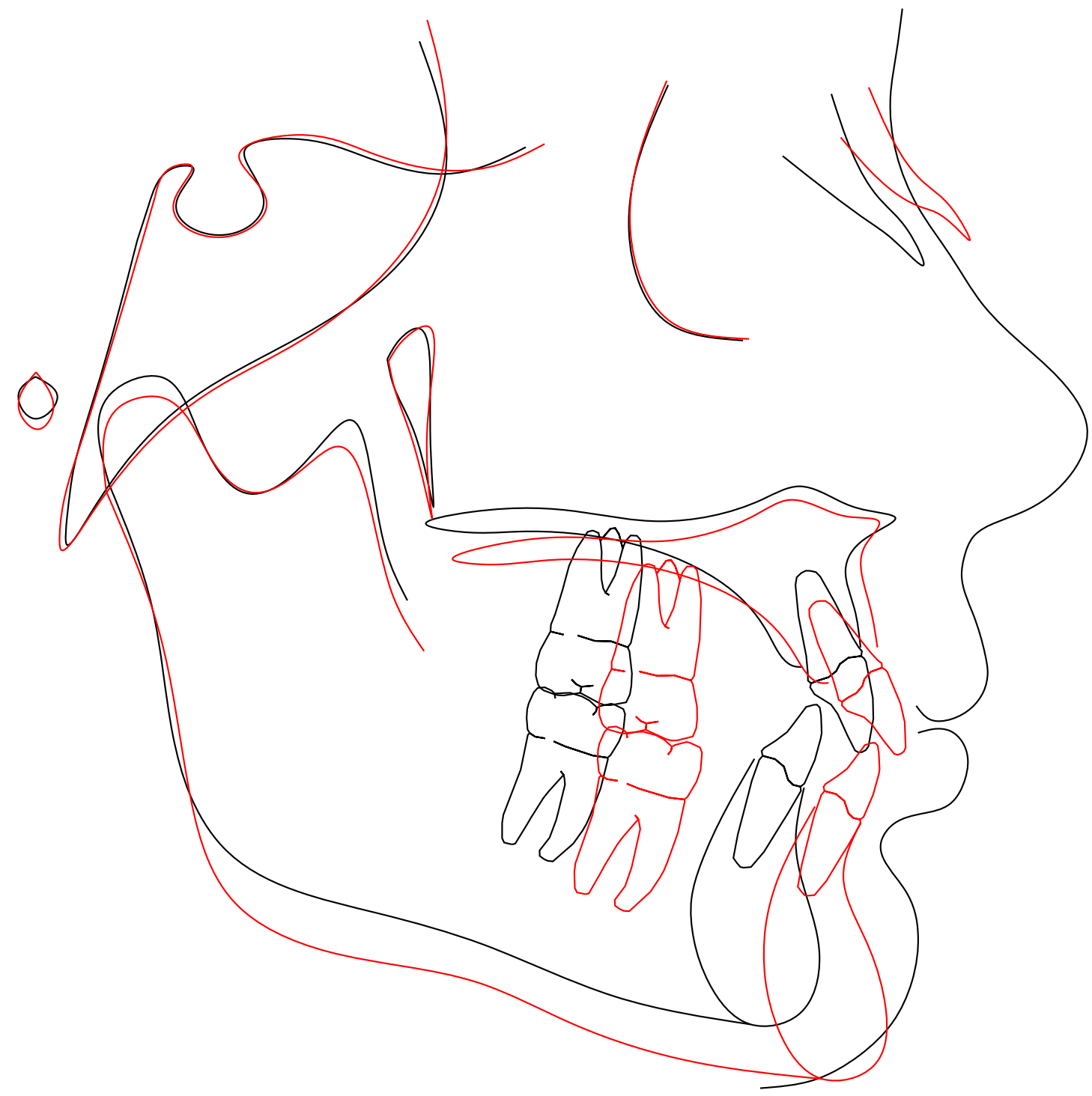

Figure F-77. Print of cephalometric tracing superimposition subject 77 (tracing is 1:1 with original radiograph). 


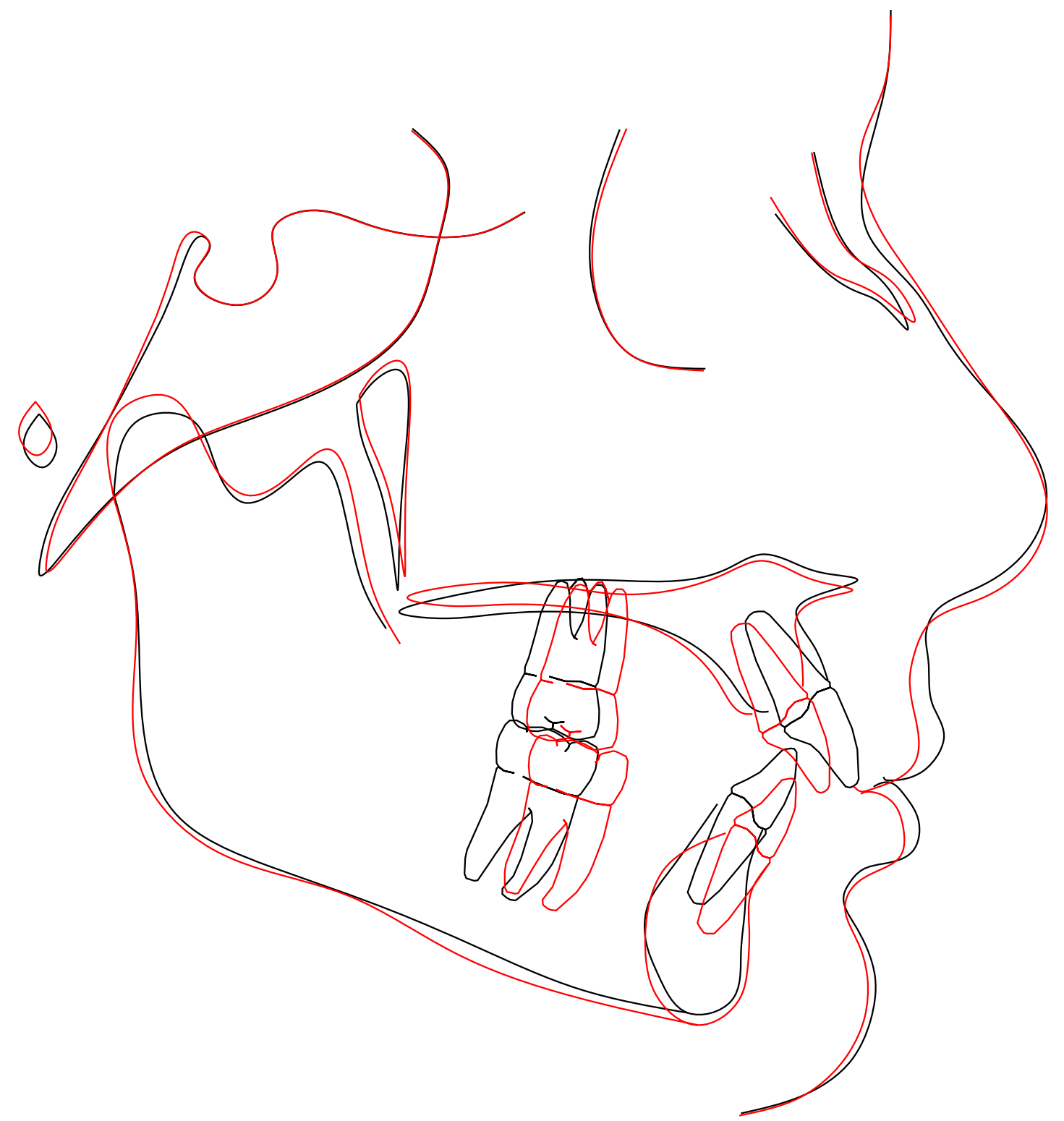

Figure F-78. Print of cephalometric tracing superimposition subject 78 (tracing is 1:1 with original radiograph). 


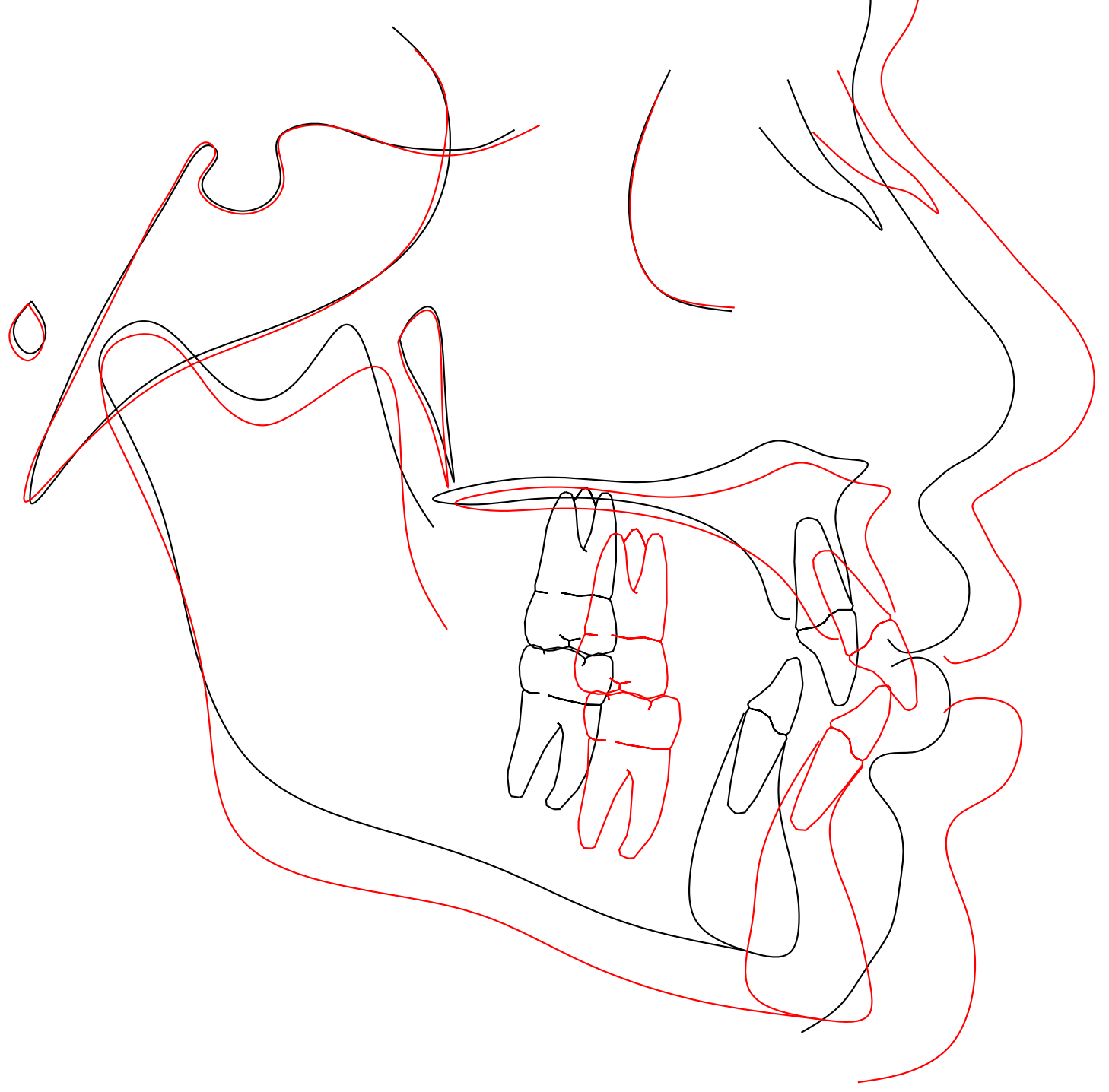

Figure F-79. Print of cephalometric tracing superimposition subject 79 (tracing is 1:1 with original radiograph). 


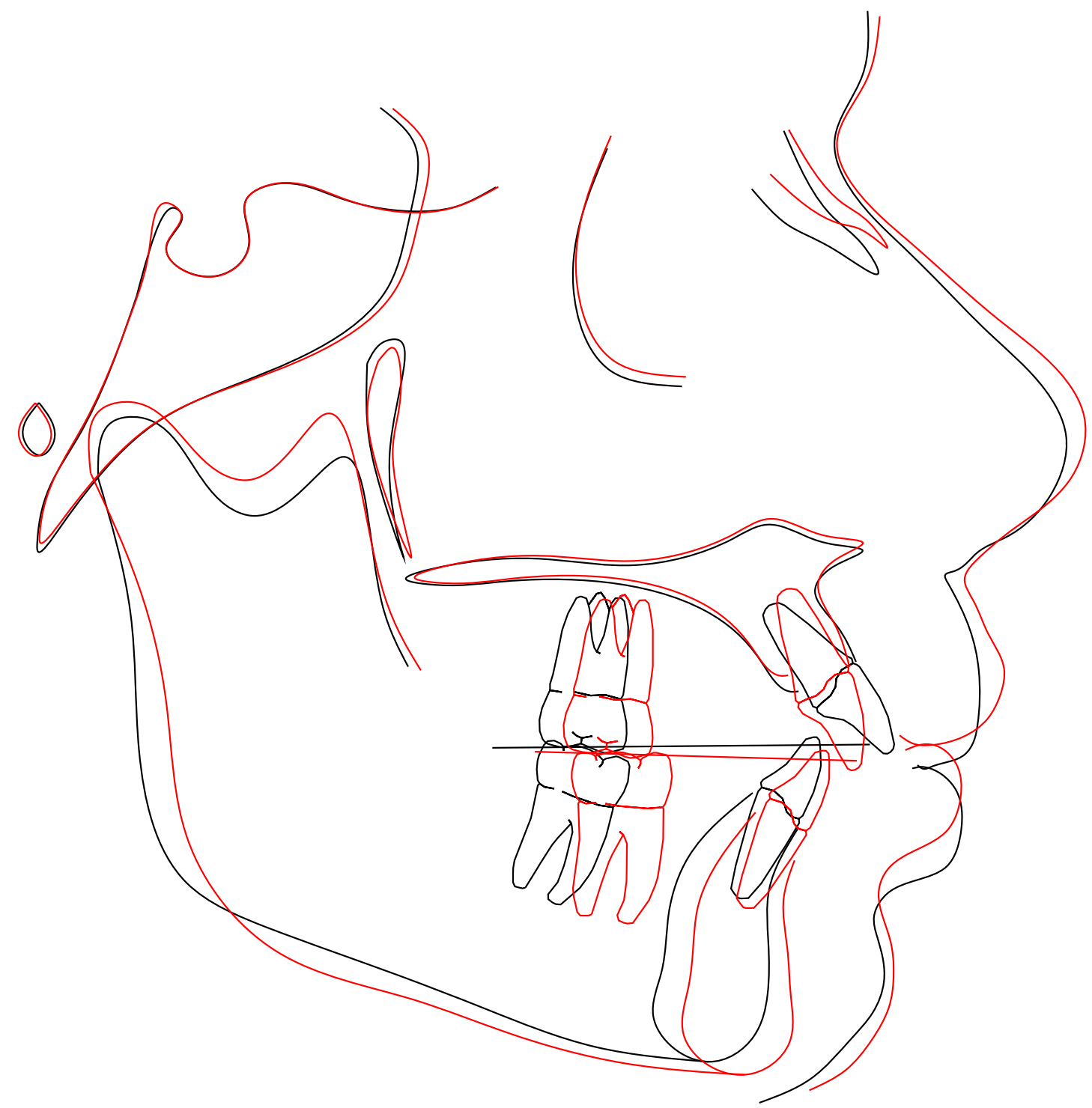

Figure F-80. Print of cephalometric tracing superimposition subject 80 (tracing is 1:1 with original radiograph). 


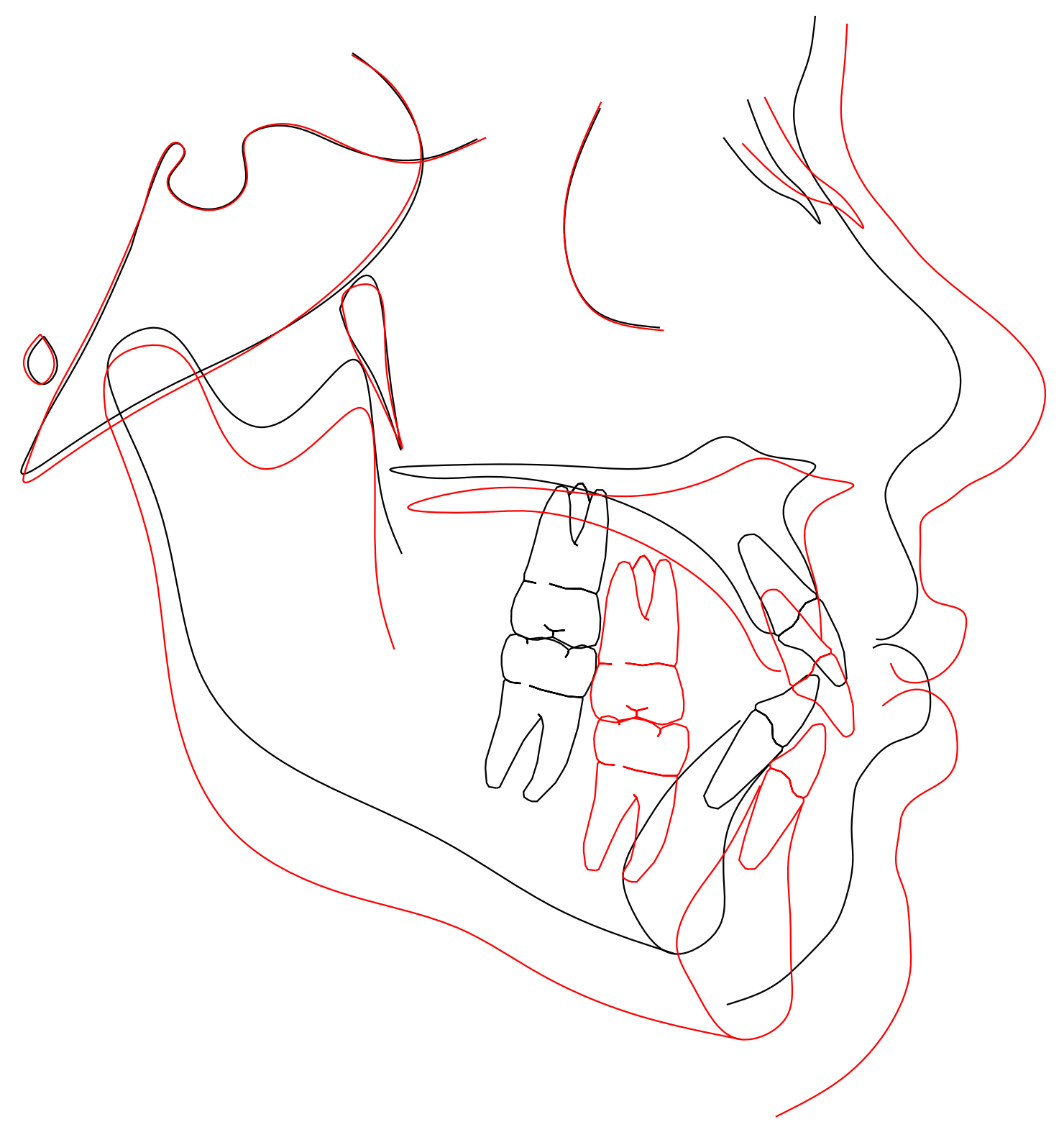

Figure F-81. Print of cephalometric tracing superimposition subject 81 (tracing is 1:1 with original radiograph). 


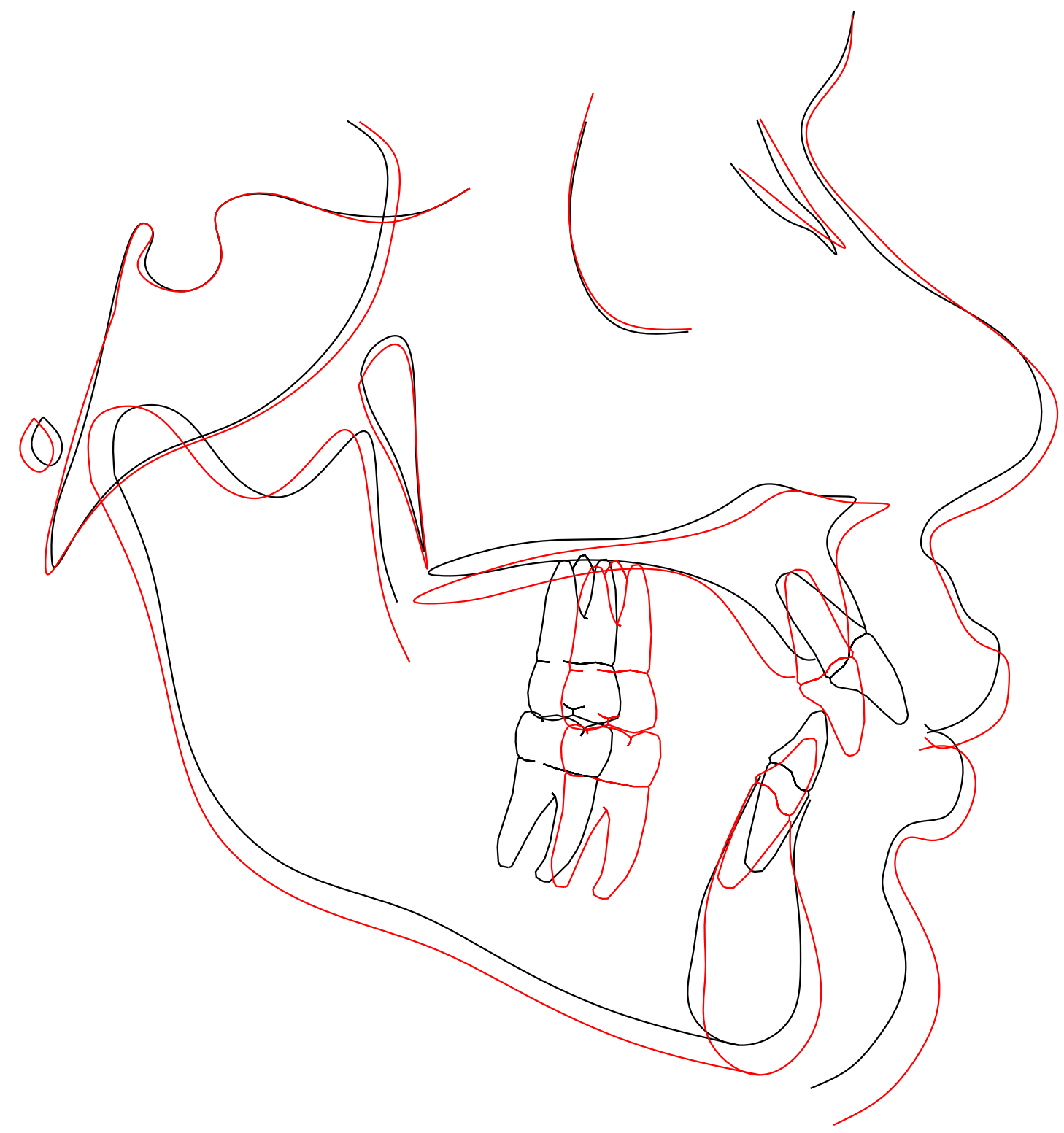

Figure F-82. Print of cephalometric tracing superimposition subject 82 (tracing is 1:1 with original radiograph). 


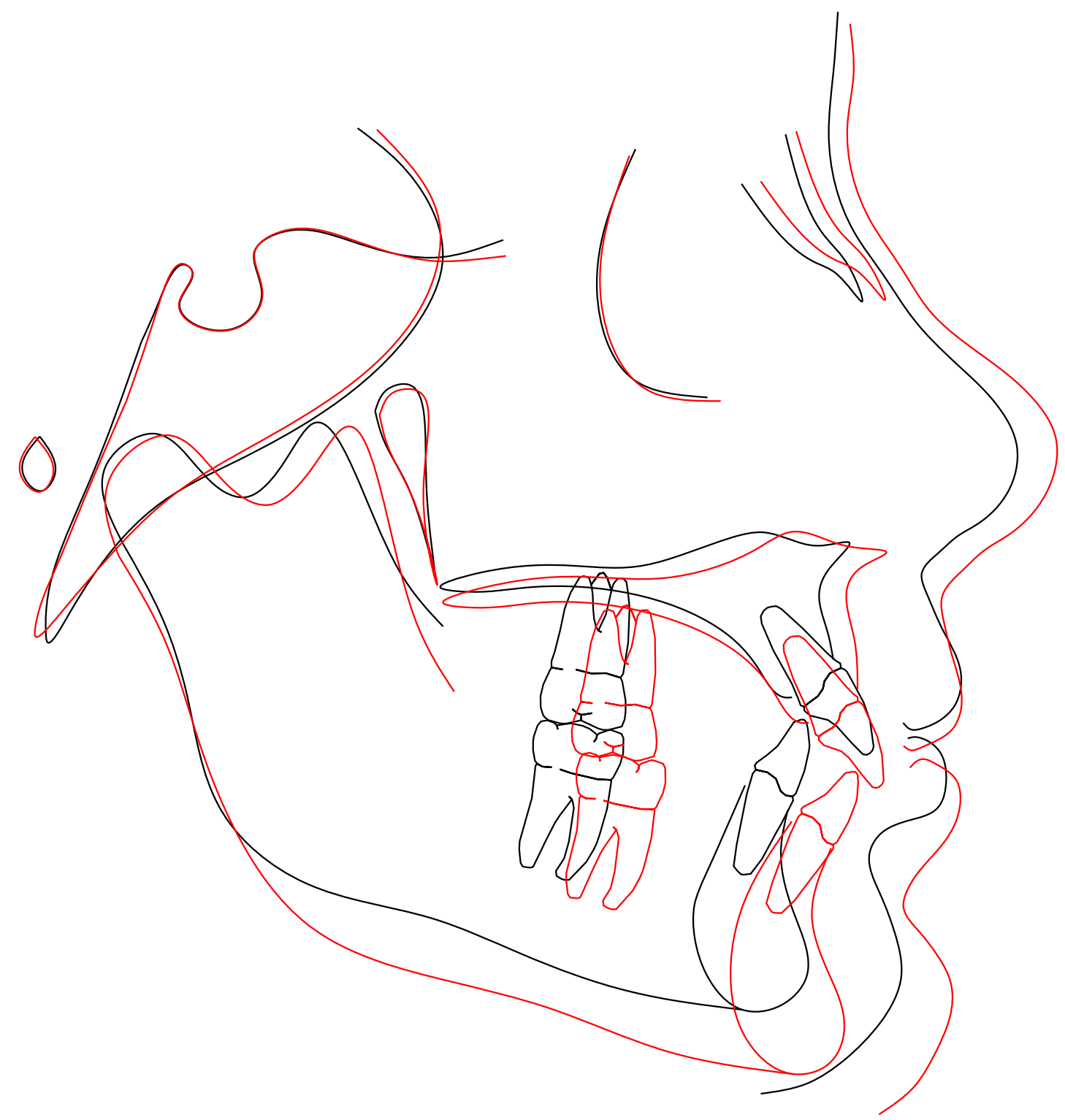

Figure F-83. Print of cephalometric tracing superimposition subject 83 (tracing is 1:1 with original radiograph). 


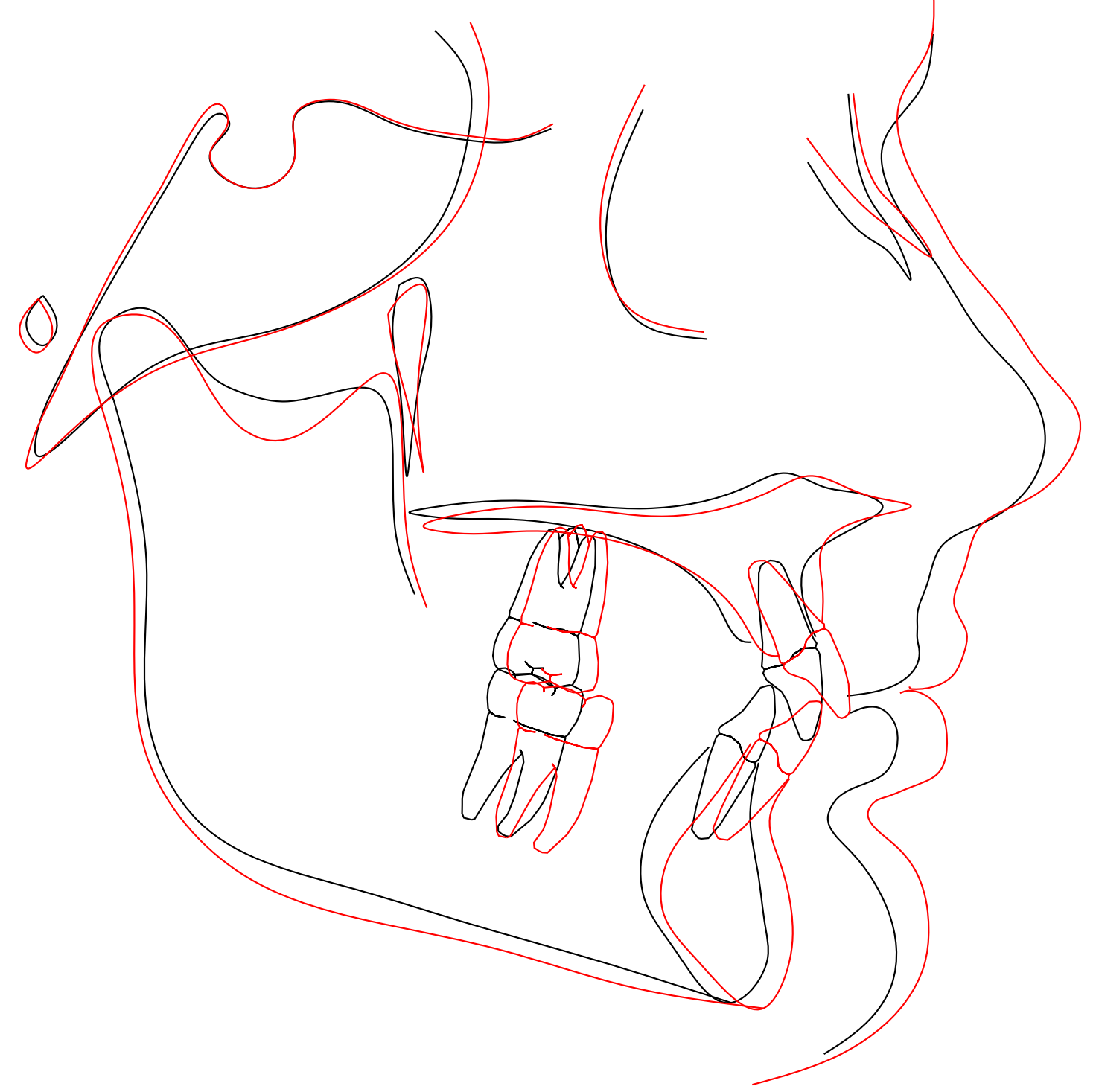

Figure F-84. Print of cephalometric tracing superimposition subject 84 (tracing is 1:1 with original radiograph). 


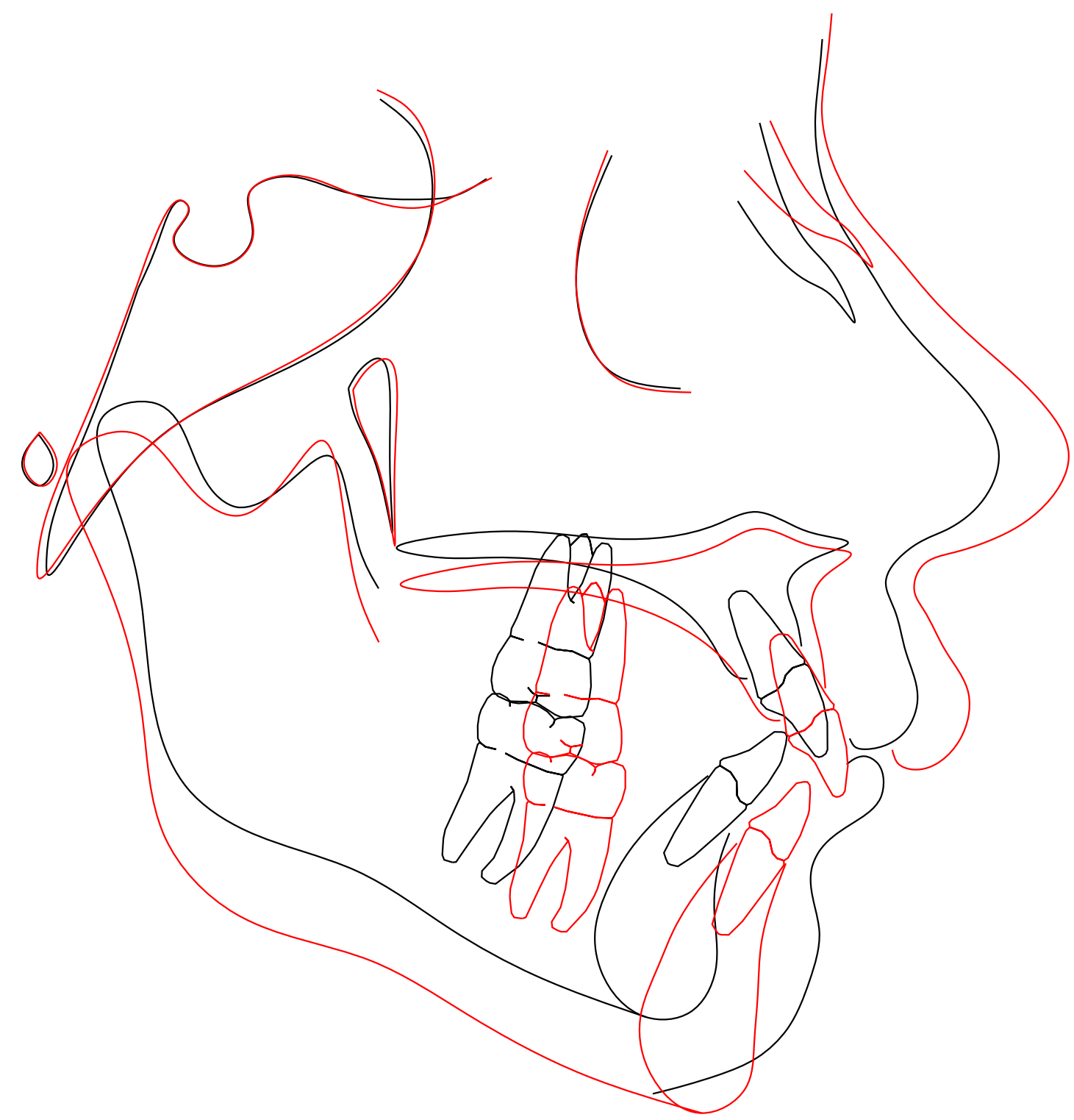

Figure F-85. Print of cephalometric tracing superimposition subject 85 (tracing is 1:1 with original radiograph). 


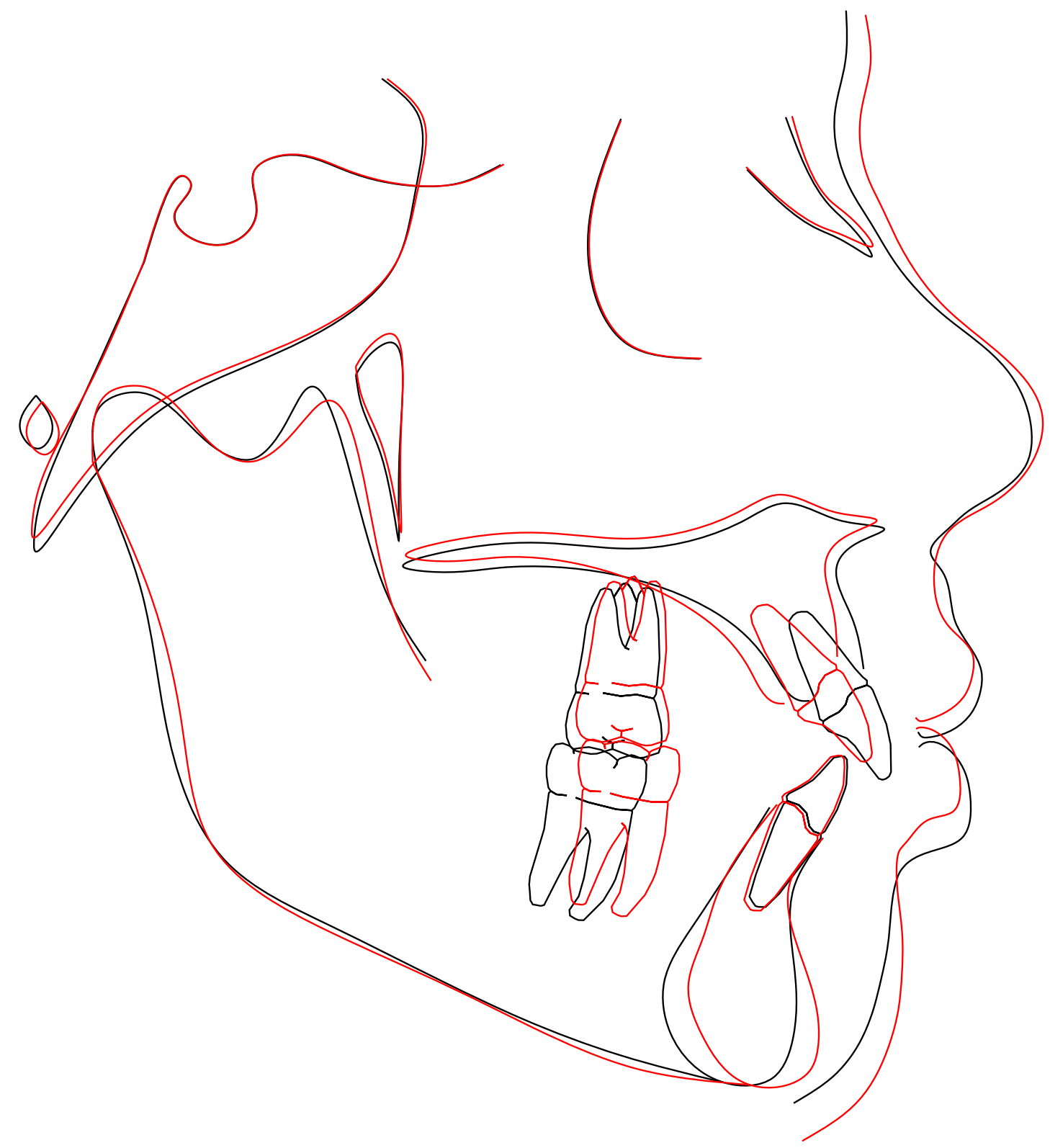

Figure F-86. Print of cephalometric tracing superimposition subject 86 (tracing is 1:1 with original radiograph). 


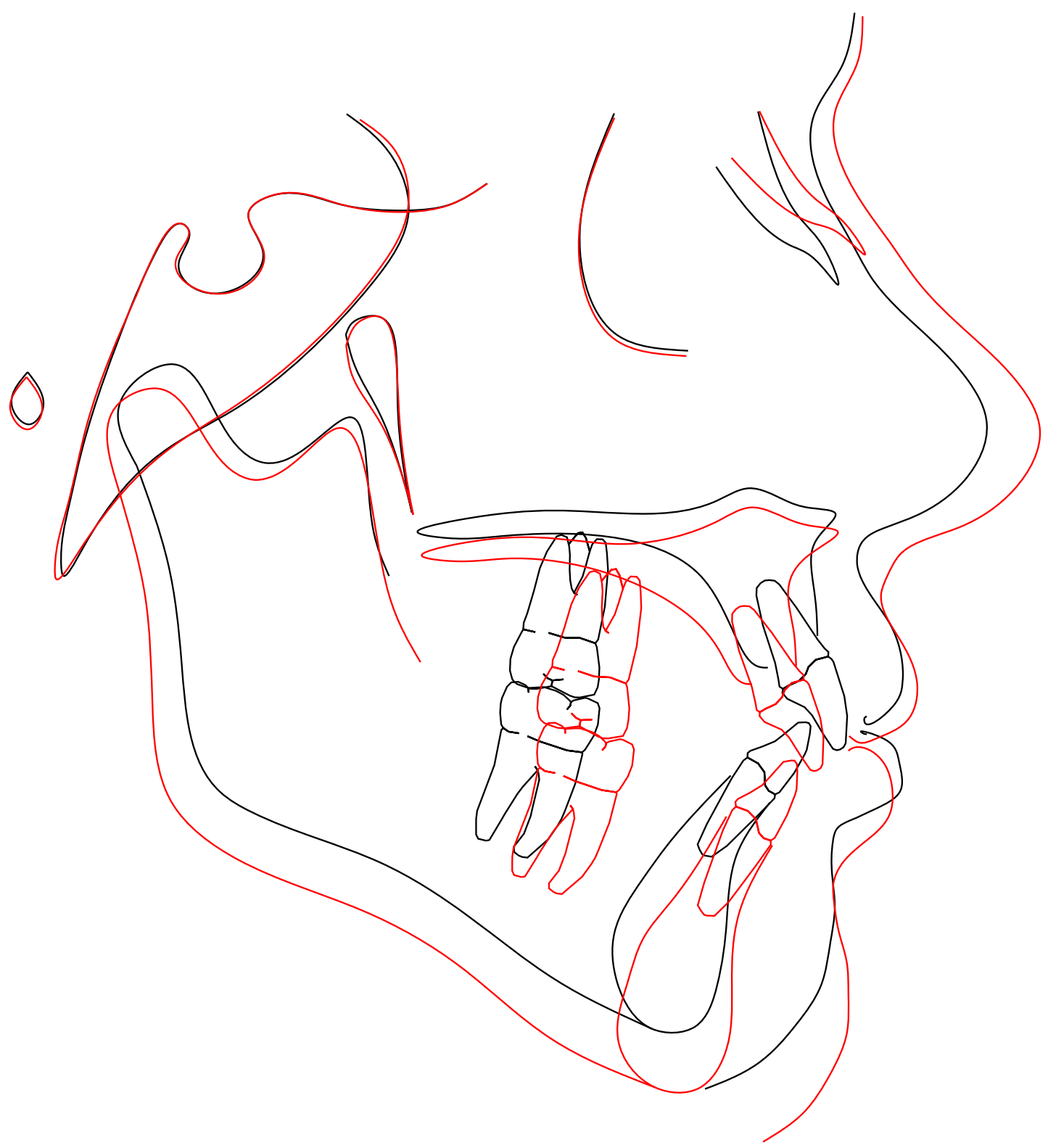

Figure F-87. Print of cephalometric tracing superimposition subject 87 (tracing is 1:1 with original radiograph). 


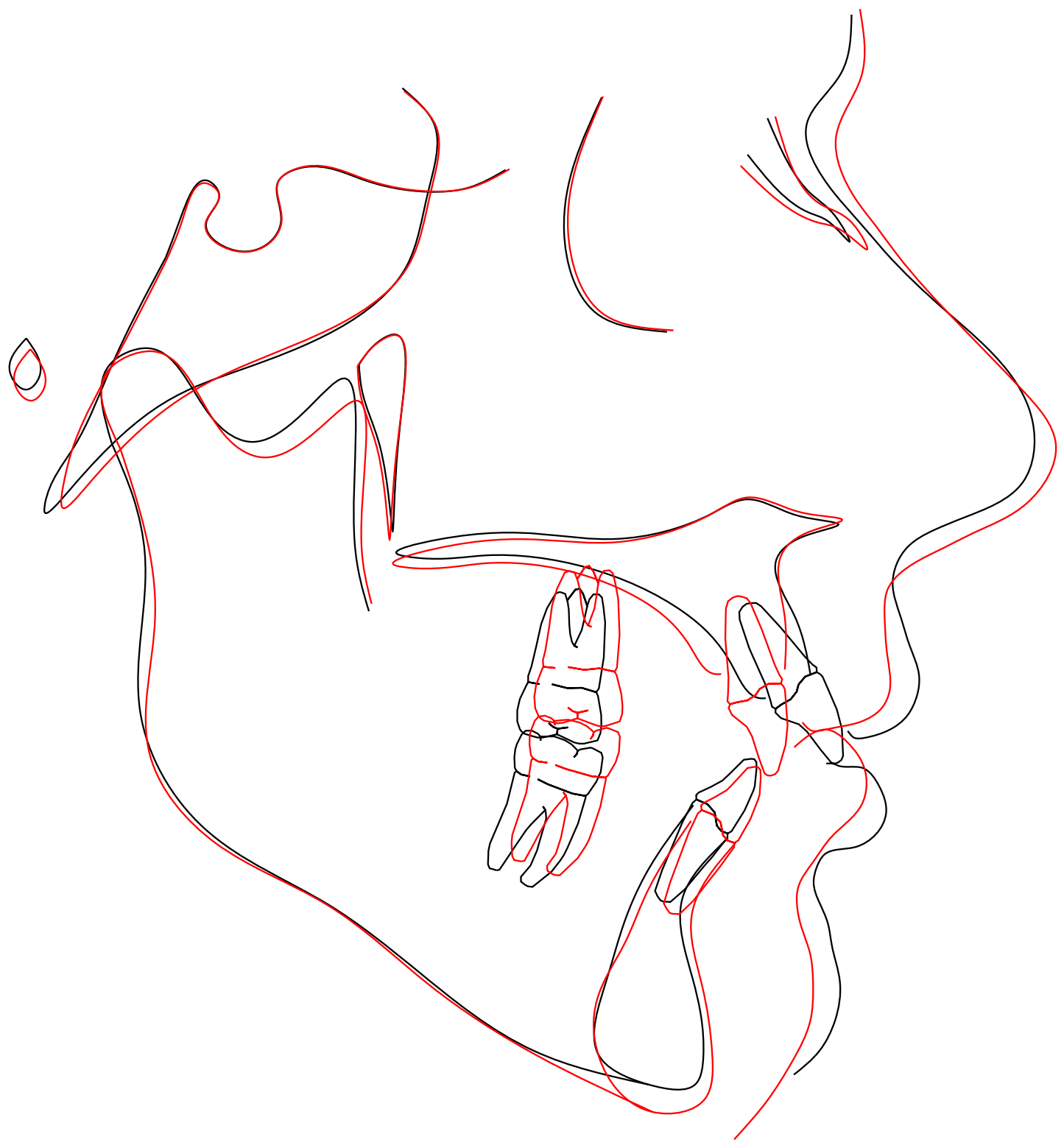

Figure F-88. Print of cephalometric tracing superimposition subject 88 (tracing is 1:1 with original radiograph). 


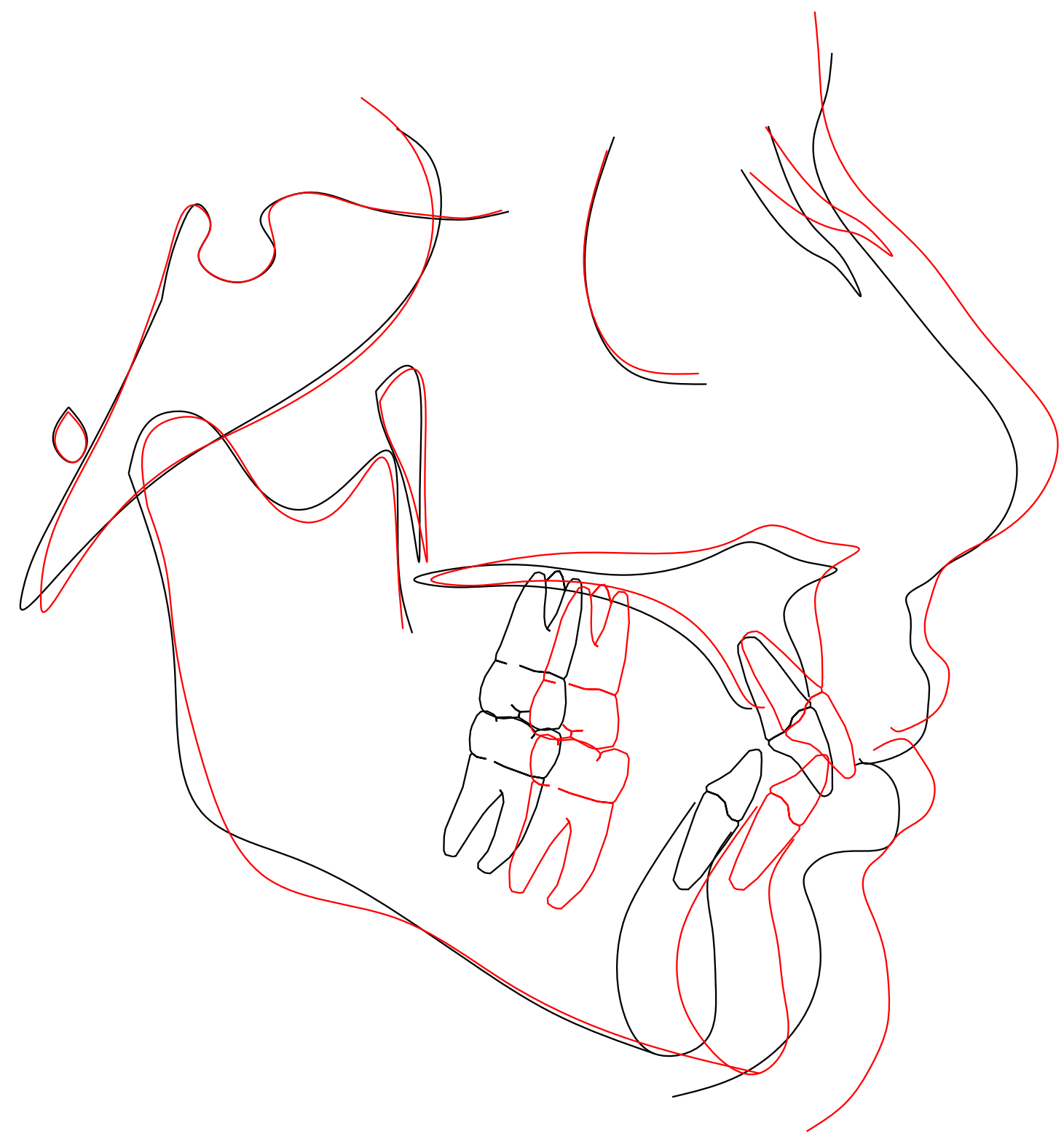

Figure F-89. Print of cephalometric tracing superimposition subject 89 (tracing is 1:1 with original radiograph). 


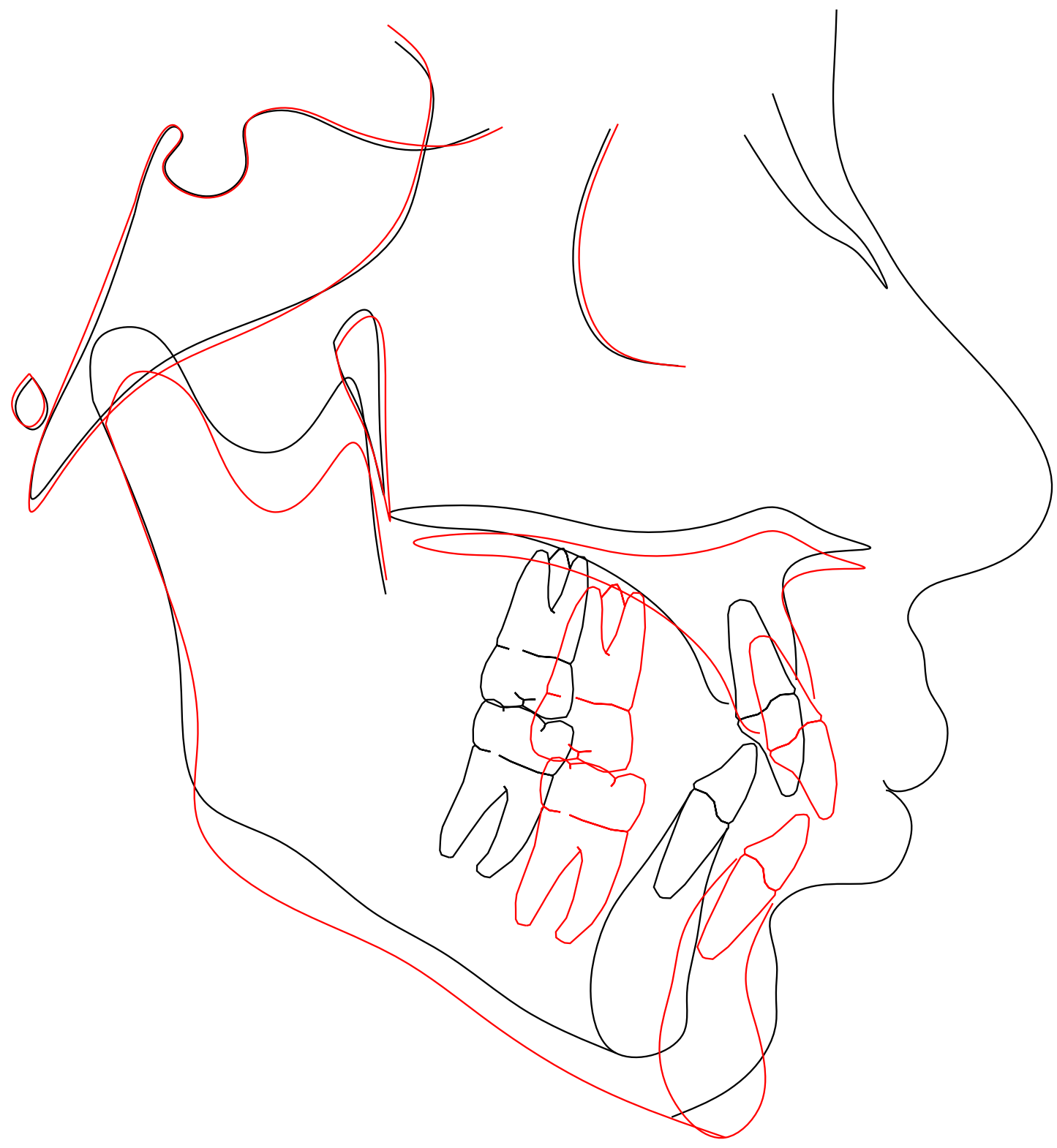

Figure F-90. Print of cephalometric tracing superimposition subject 90 (tracing is 1:1 with original radiograph). 


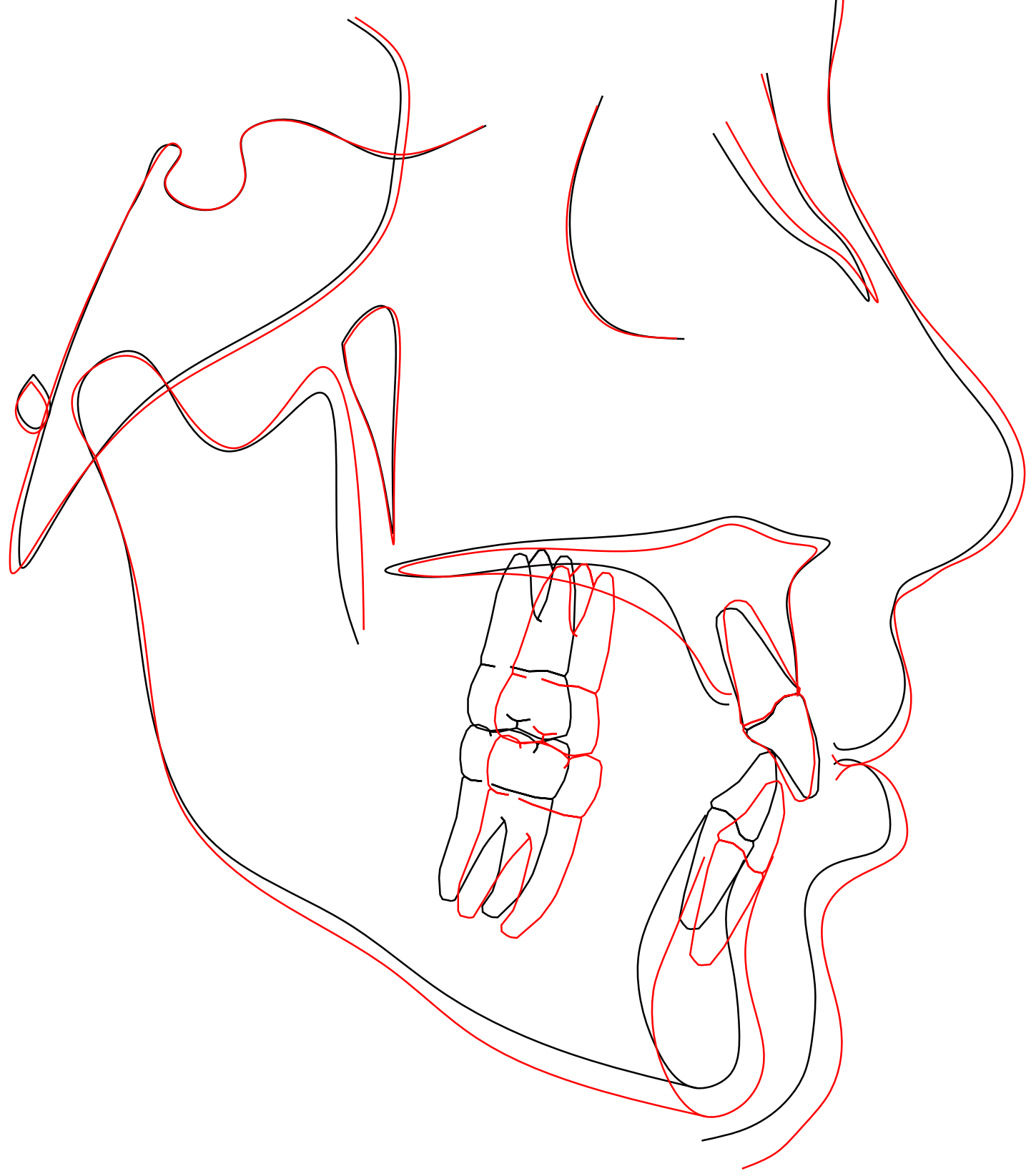

Figure F-91. Print of cephalometric tracing superimposition subject 91 (tracing is 1:1 with original radiograph). 


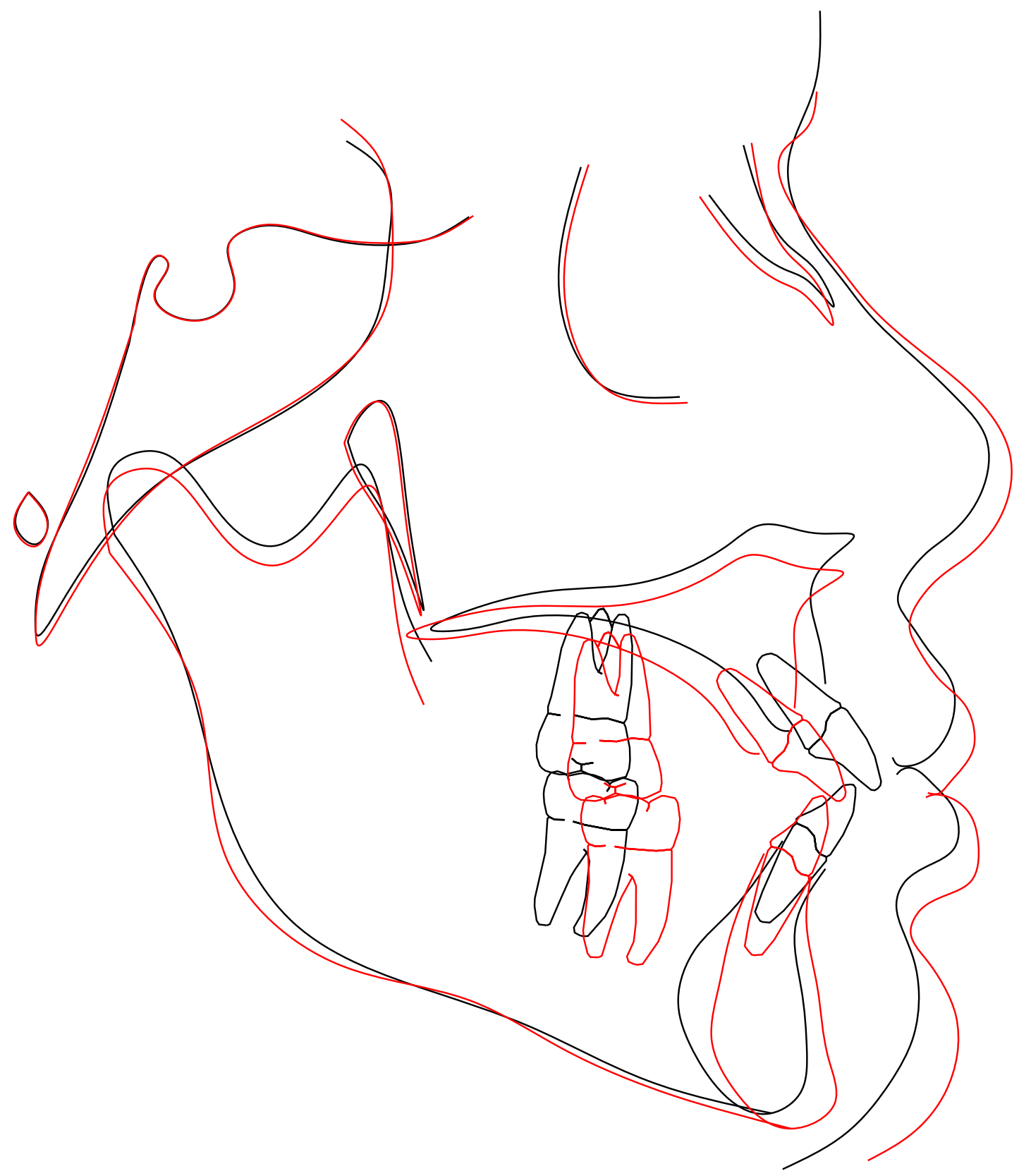

Figure F-92. Print of cephalometric tracing superimposition subject 92 (tracing is 1:1 with original radiograph). 


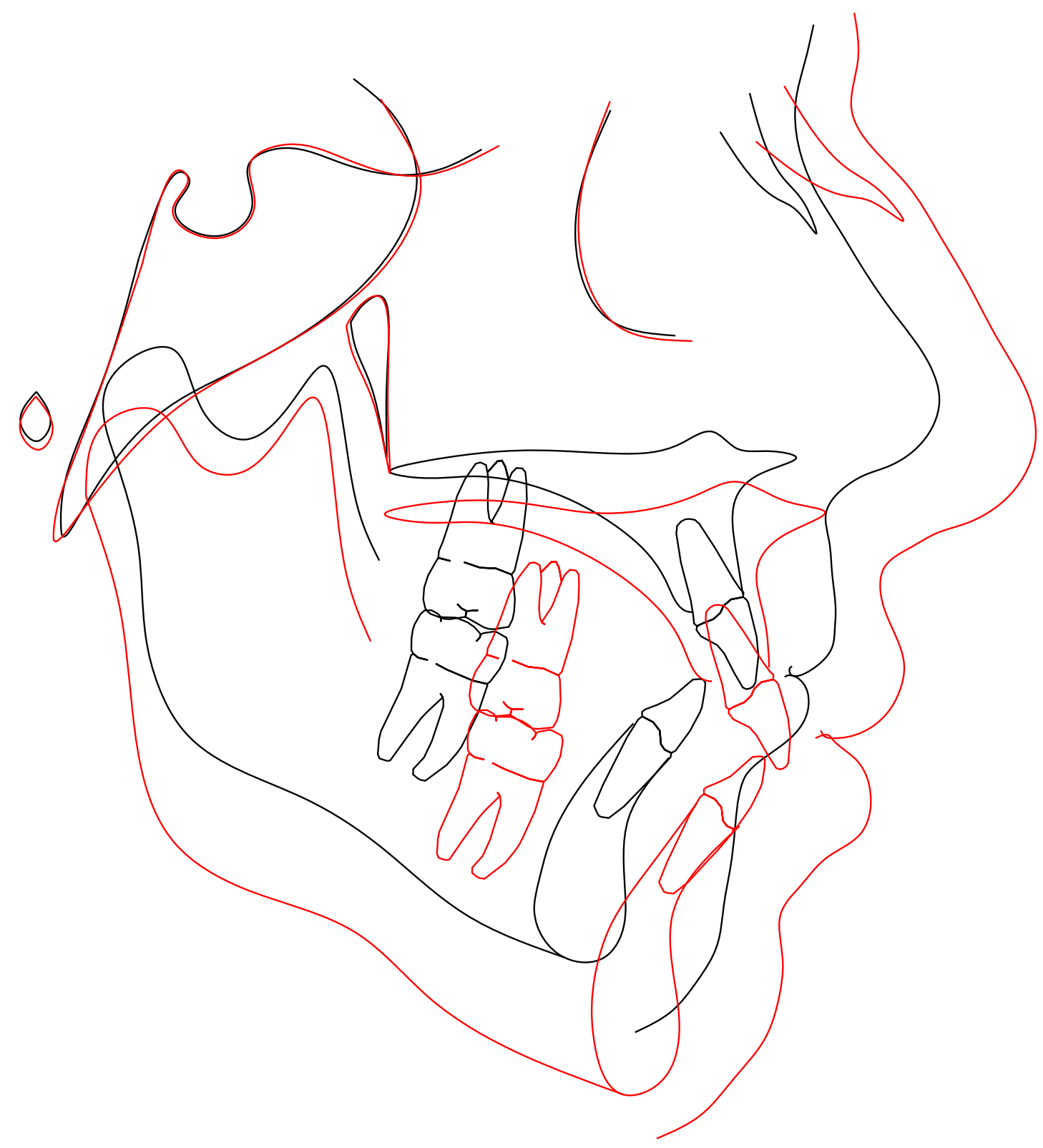

Figure F-93. Print of cephalometric tracing superimposition subject 93 (tracing is 1:1 with original radiograph). 


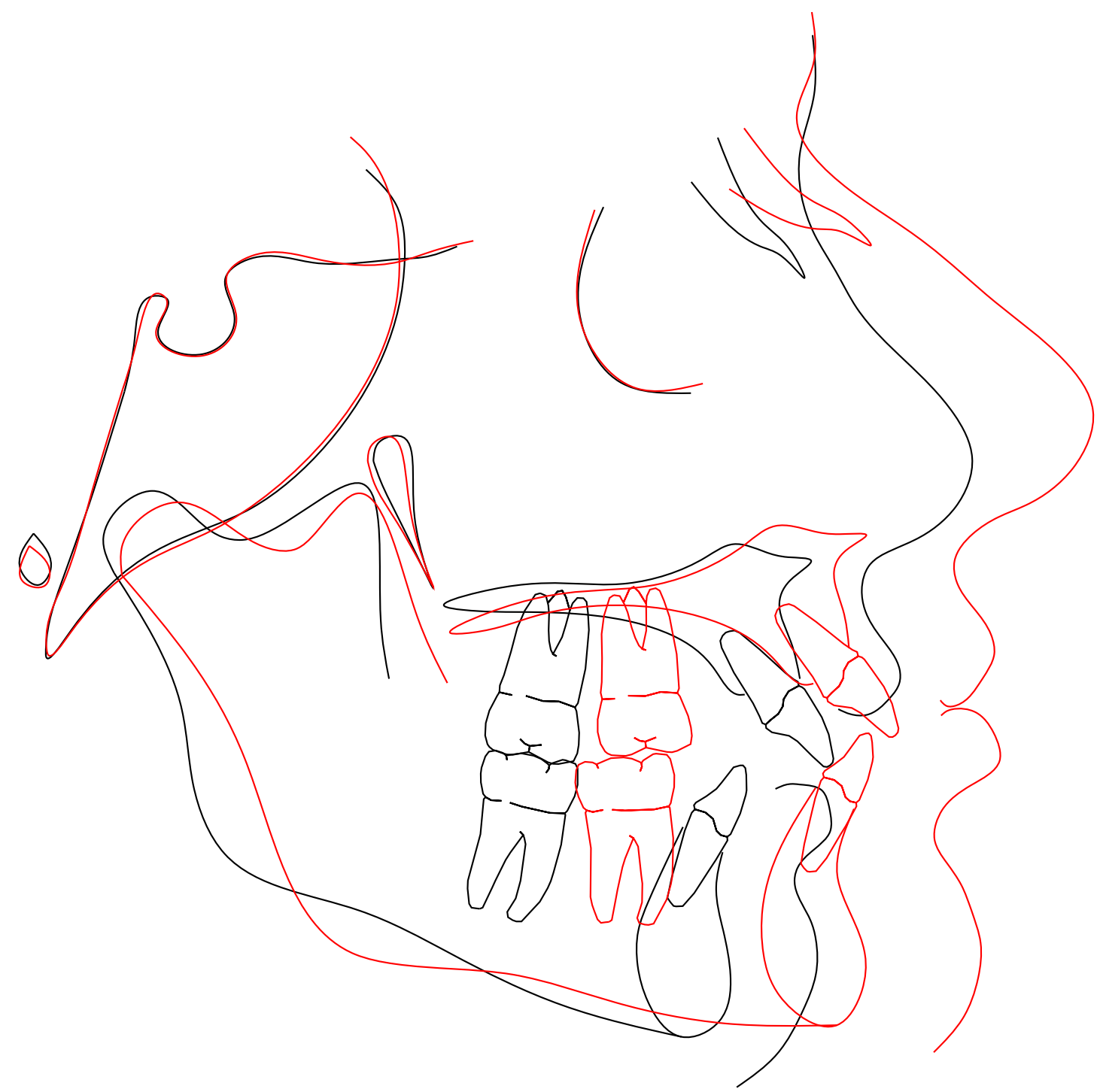

Figure F-94. Print of cephalometric tracing superimposition subject 94 (tracing is 1:1 with original radiograph). 


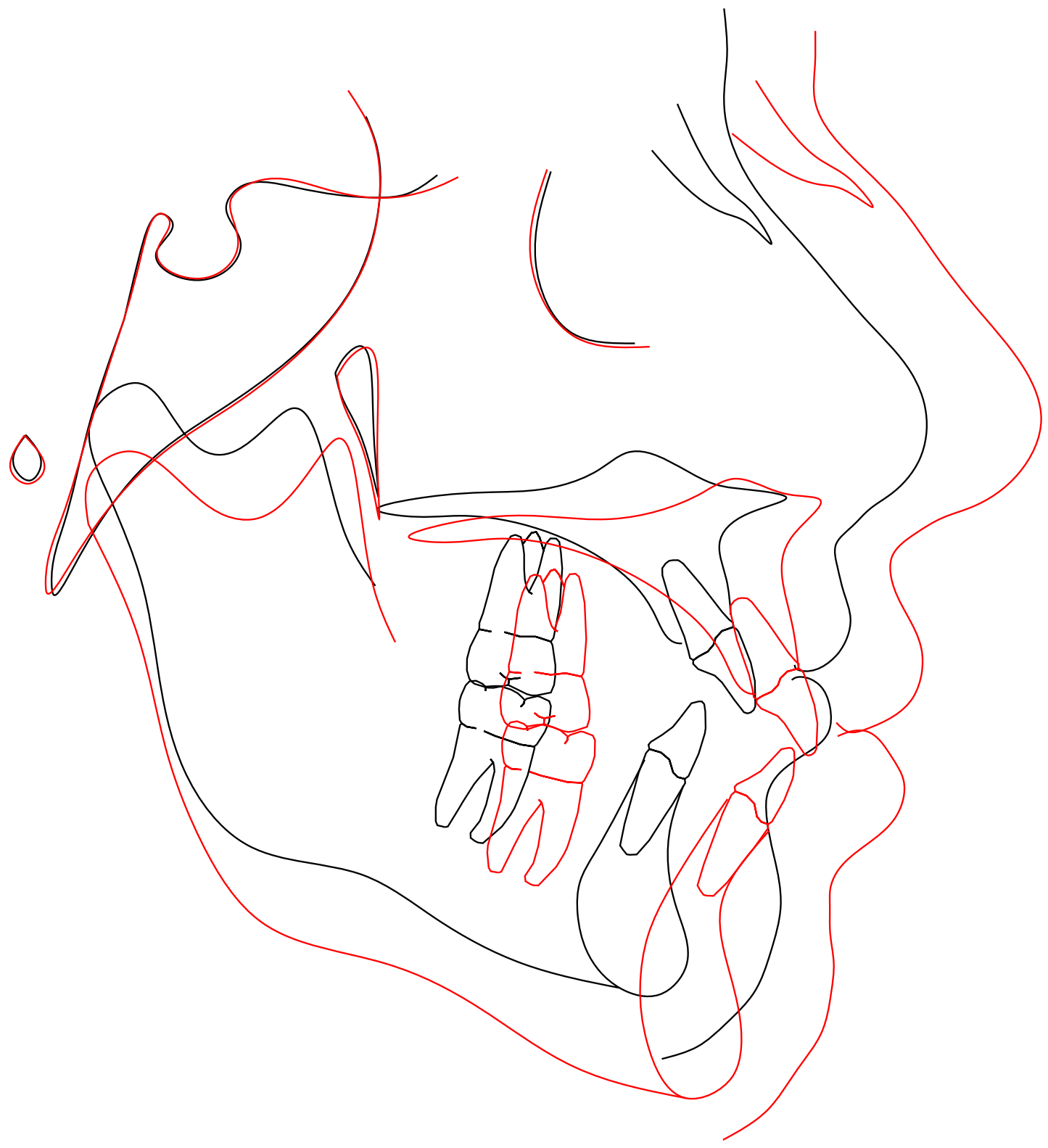

Figure F-95. Print of cephalometric tracing superimposition subject 95 (tracing is 1:1 with original radiograph). 


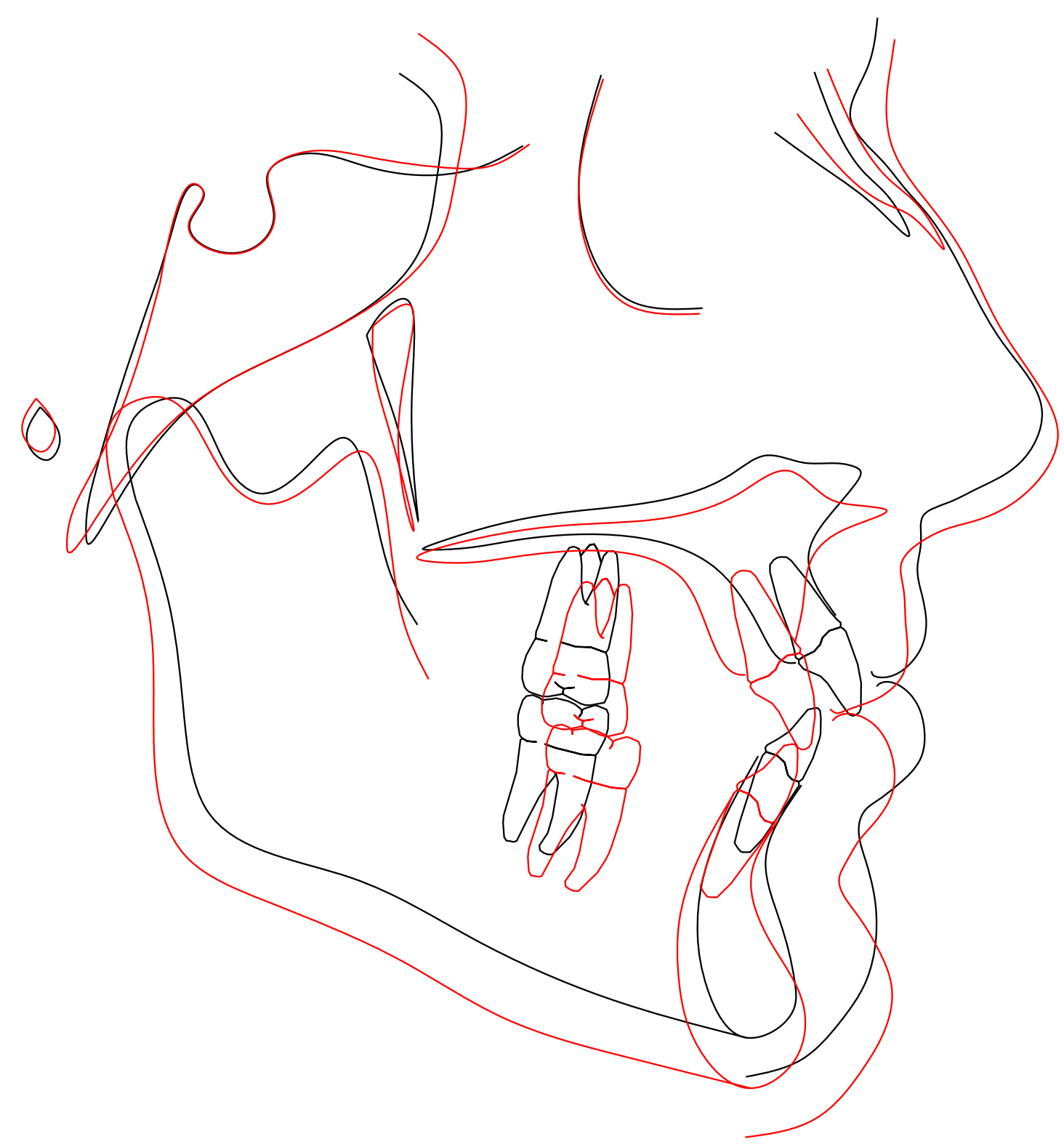

Figure F-96. Print of cephalometric tracing superimposition subject 96 (tracing is 1:1 with original radiograph). 


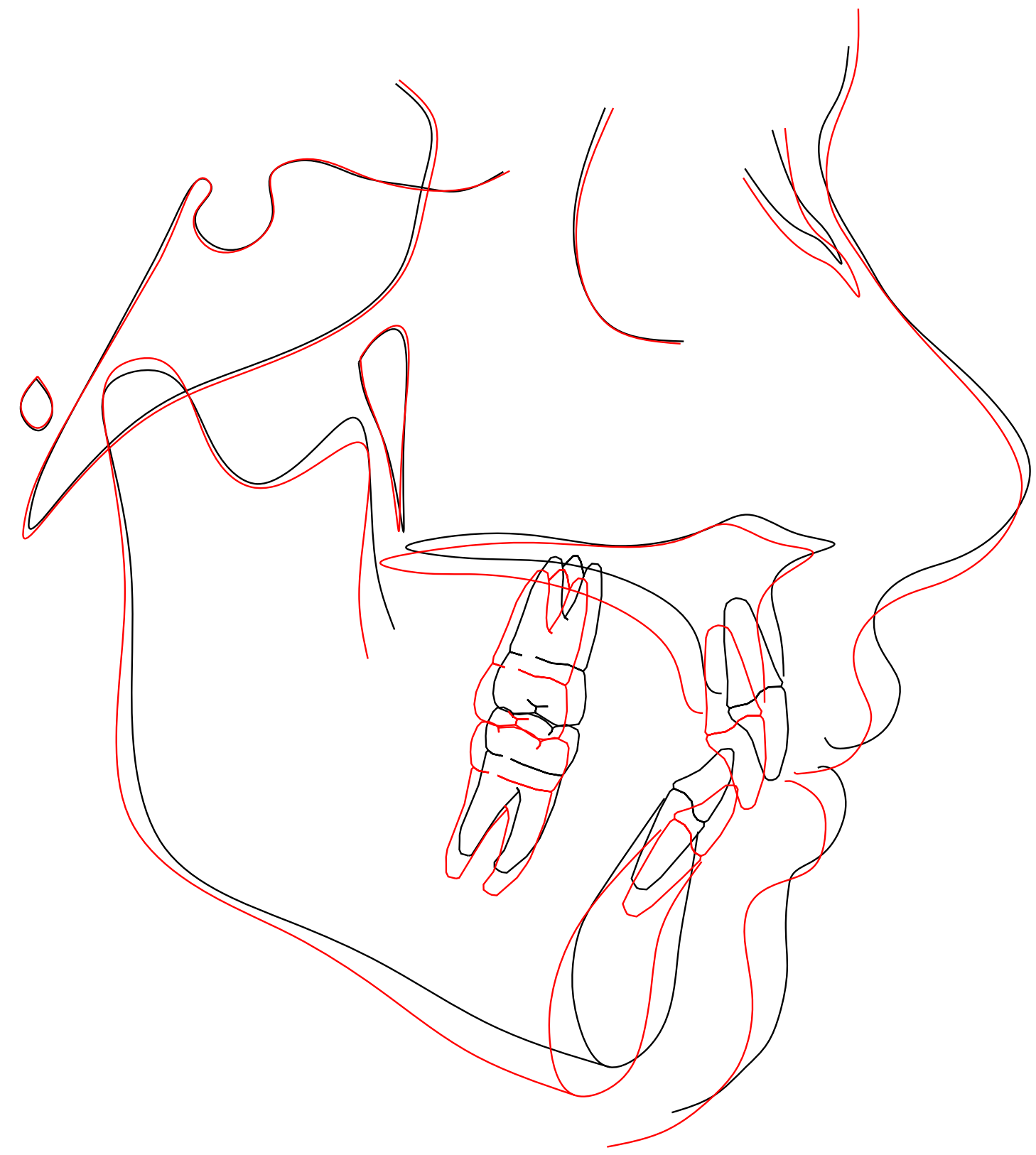

Figure F-97. Print of cephalometric tracing superimposition subject 97 (tracing is 1:1 with original radiograph). 


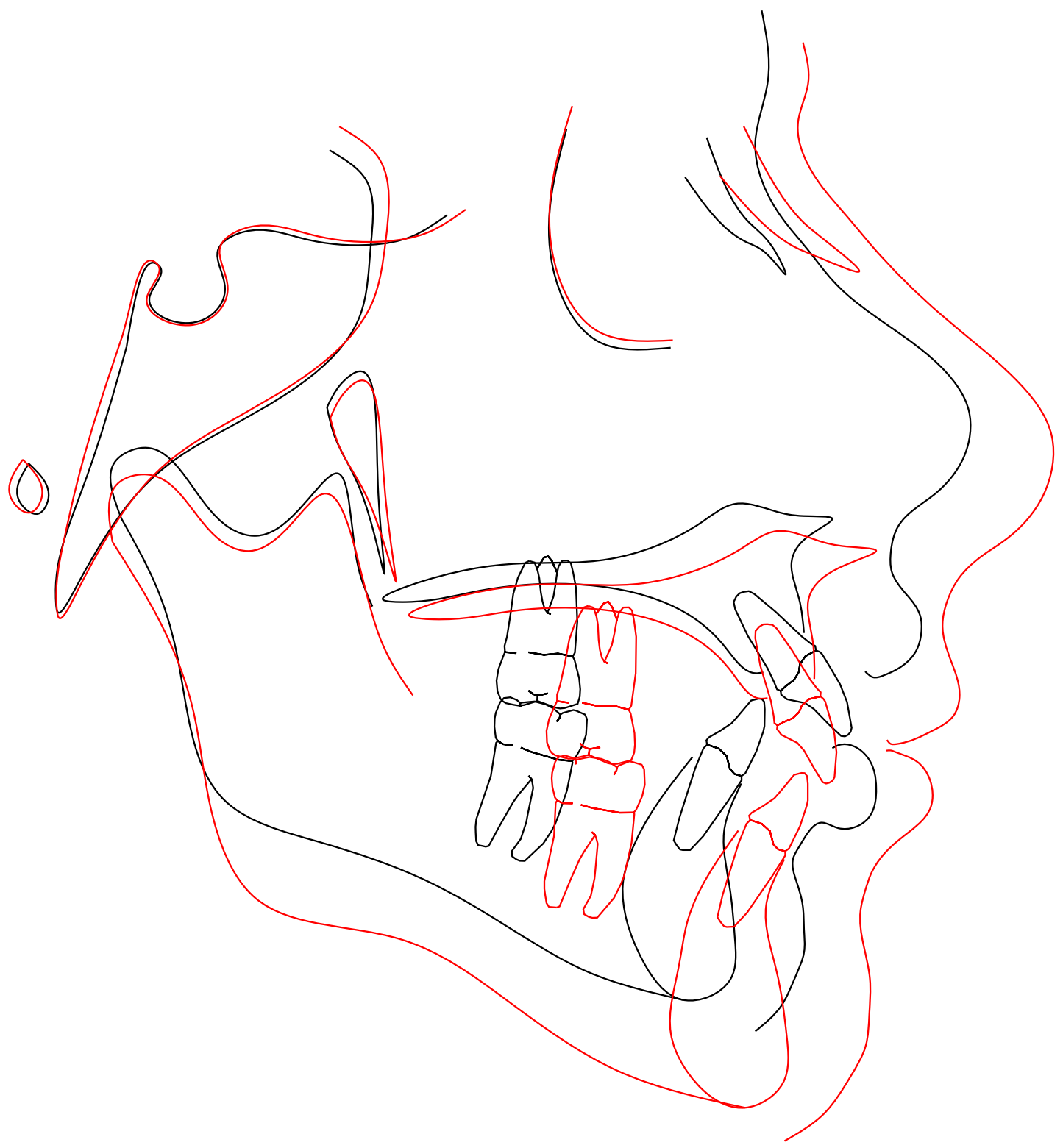

Figure F-98. Print of cephalometric tracing superimposition subject 98 (tracing is 1:1 with original radiograph). 


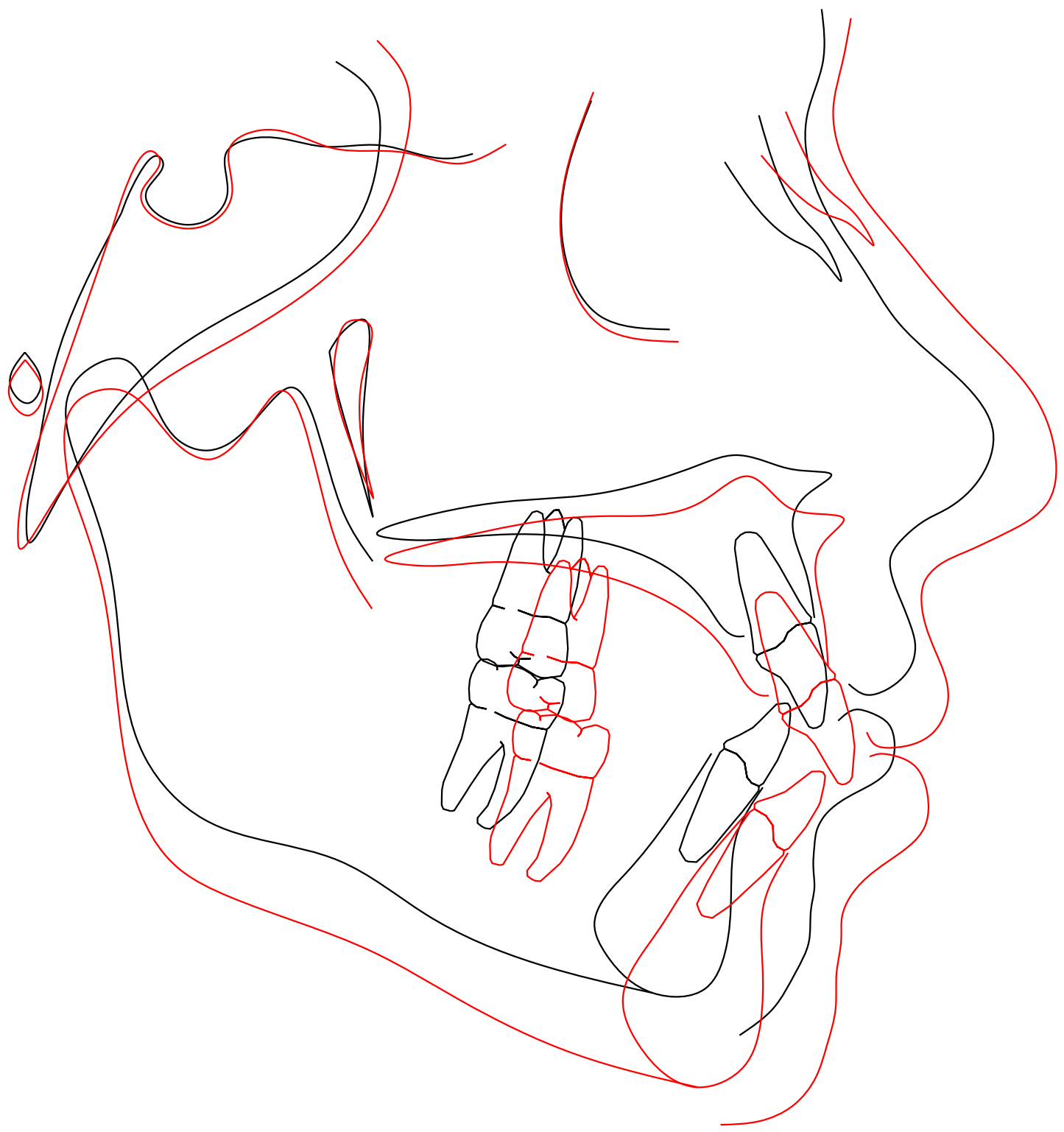

Figure F-99. Print of cephalometric tracing superimposition subject 99 (tracing is 1:1 with original radiograph). 


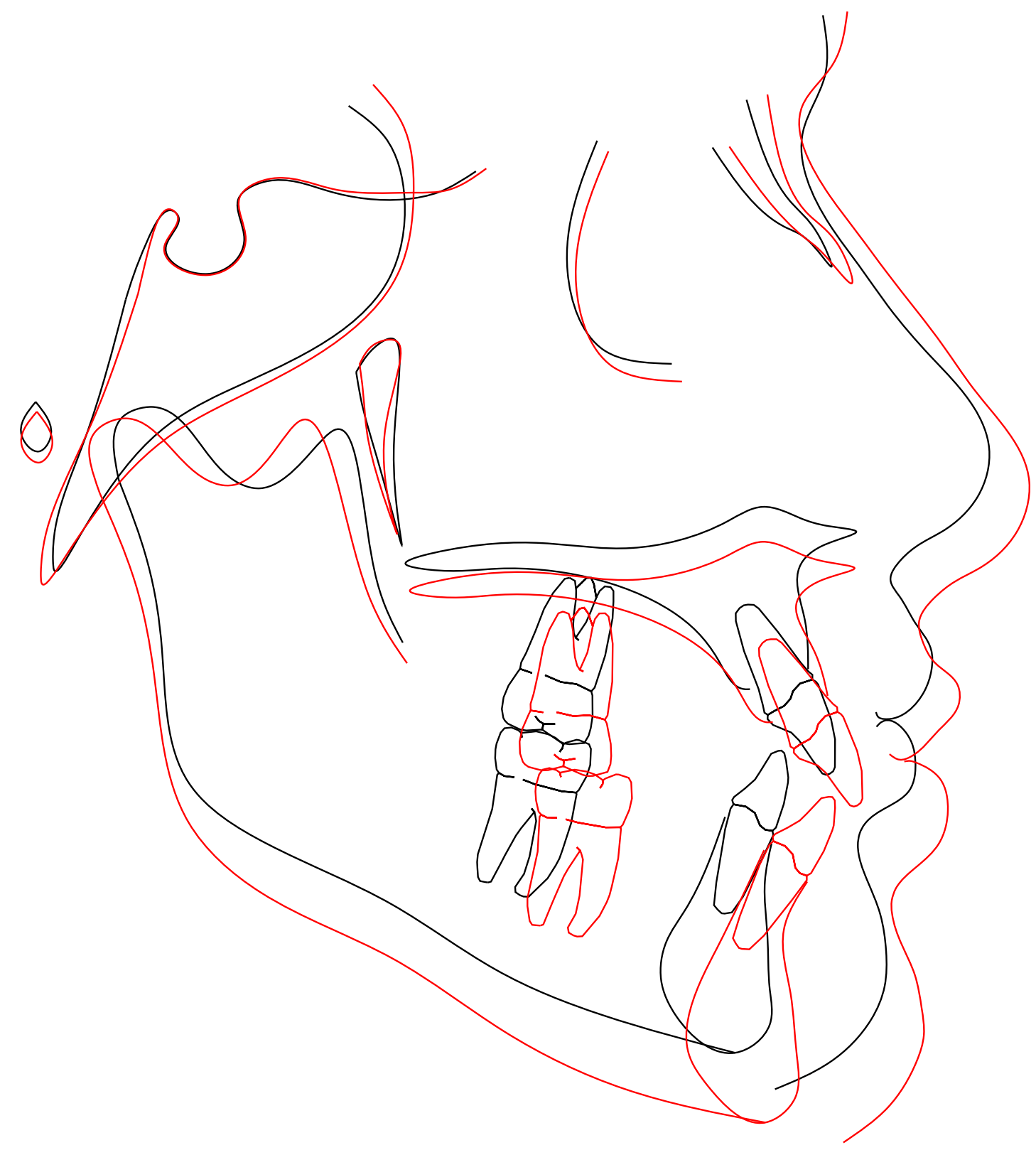

Figure F-100. Print of cephalometric tracing superimposition subject 100 (tracing is 1:1 with original radiograph). 


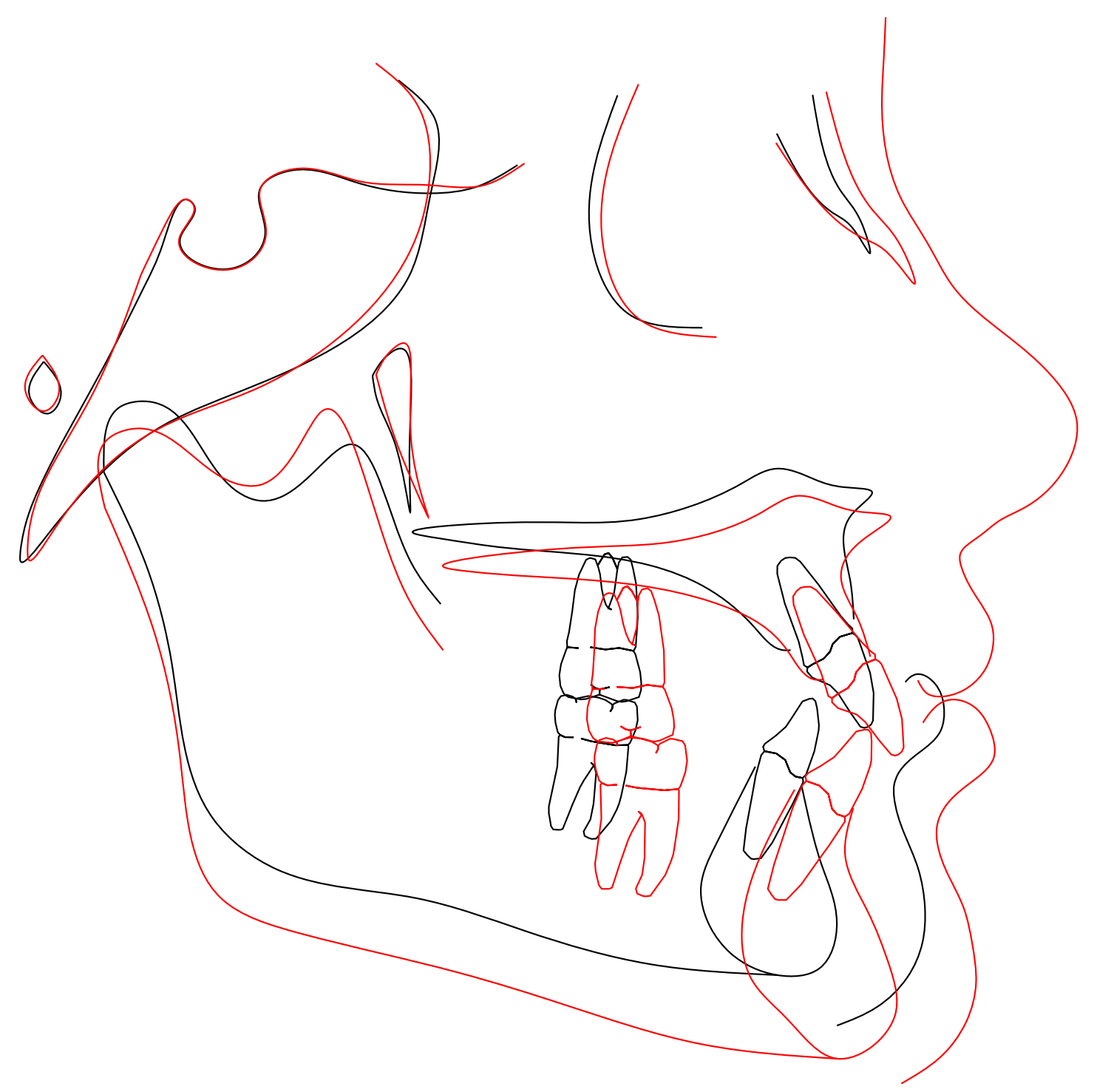

Figure F-101. Print of cephalometric tracing superimposition subject 101 (tracing is $1: 1$ with original radiograph). 


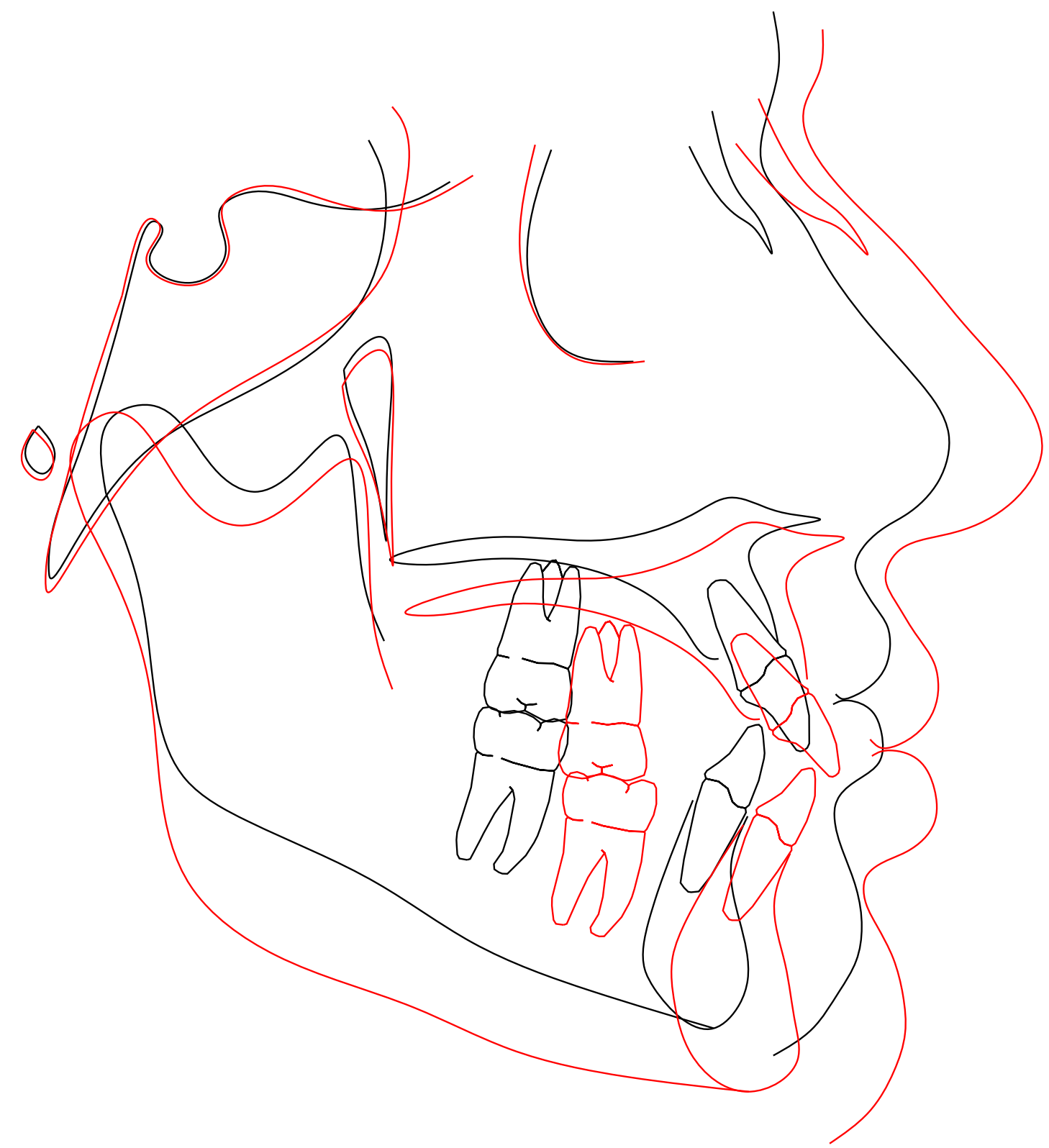

Figure F-102. Print of cephalometric tracing superimposition subject 102 (tracing is 1:1 with original radiograph). 


\section{VITA}

Tiffany Rachelle McClaran, the daughter of Dr. John and Martha Wilhoit, was born in Pikeville, Tennessee, on October 28, 1980. Tiffany graduated from The Baylor School, in Chattanooga, Tennessee, in 1999 and attended Middle Tennessee State University where she received a Bachelor of Science degree in Allied Health. She received her dental training and a Doctor of Dental Surgery degree from The University of Tennessee Health Science Center, Memphis, in May of 2007. In August of 2007, she entered The University of Tennessee Health Science Center as a graduate student in the Department of Orthodontics and is expected to receive her Master of Dental Science in May 2010. Tiffany and her husband, Matt McClaran, plan to live in Franklin, Tennessee, upon graduation. 